\title{
Indole- and Pyrrole-BX: Bench-Stable Hypervalent lodine Reagents for Heterocycle Umpolung
}

\author{
Paola Caramenti, Stefano Nicolai and Jerome Waser*
}

\begin{abstract}
The one-step synthesis of the bench-stable hypervalent iodine reagents IndoleBX and PyrroleBX using mild Lewis acid catalyzed conditions is reported. The new reagents are stable up to $150{ }^{\circ} \mathrm{C}$ and were applied in the $\mathrm{C}-\mathrm{H}$ arylation of unactivated arenes using either rhodium or ruthenium catalysts. A broad range of heterocyclic systems of high interest for synthetic and medicinal chemistry was accessed in high yields. The developed $\mathrm{C}-\mathrm{H}$ functionalization could not be achieved using reported reagents or methods, highlighting the unique reactivity of Indole- and Pyrrole-BX.
\end{abstract}

Pyrrole and indole heterocycles are omnipresent in natural and synthetic biologically active compounds and have found countless applications in the pharmaceutical and agrochemical industries. ${ }^{[1]}$ Therefore, synthetic chemists have invested unceasing efforts towards their synthesis and functionalization. ${ }^{[2]}$ As indoles and pyrroles are highly nucleophilic, the broad majority of functionalization methods is based on reactions with electrophiles, which limits the structural diversity of compounds in medicinal chemistry studies. In order to allow indoles and pyrroles to react with nucleophiles and discover new chemical space, an Umpolung of the innate reactivity of the heterocycles would be highly useful. However, accessing electrophilic indole and pyrrole synthons constitutes a formidable challenge. Most approaches rely on the substitution of the heterocycles with one or several electron-withdrawing groups or on reactive electrophilic indolyl intermediates generated in situ. ${ }^{[3]}$ More stable halogenated indoles have been used only in cross-coupling reactions with activated partners. ${ }^{[4]}$ Clearly, the development of better indole and pyrrole electrophilic synthons combining high reactivity with enhanced stability is needed.

In this context, hypervalent iodine reagents are recognized for their high reactivity, which can lead to the formal Umpolung of functional groups. ${ }^{[5]}$ The introduction of reactive yet stable aryl iodonium salts (Scheme 1A) has led to the development of broadly applicable arylation reactions. ${ }^{[6]}$ Nevertheless, there are only few reports on indole and pyrrole-based iodonium salts. Pioneering works by Neiland, Kost, Moriarty and co-workers required a multi-step procedure via a potentially explosive betaine intermediate to access $\mathrm{NH}$ unprotected indole iodonium salts. ${ }^{[7]}$

[a] Paola Caramenti, Dr. Stefano Nicolai and Prof. Dr. Jerome Waser Laboratory of Catalysis and Organic Synthesis Ecole Polytechnique Fédérale de Lausanne

EPFL SB ISIC LCSO, BCH 4306, 1015 Lausanne $(\mathrm{CH})$

Fax: (+)41216939700

E-mail: jerome.waser@epfl.ch

Supporting information for this article is given via a link at the end of the document.
Recently, more stable indole and pyrrole iodonium salts were reported by Moriyama and co-workers ${ }^{[8]}$ and Kita and coworkers ${ }^{[9]}$ respectively, based on the introduction of an electronwithdrawing group on the nitrogen. The electrophilic character of these reagents was used for direct reactions with nucleophiles, such as alkyl lithium reagents, ${ }^{[7 c]}$ azides, ${ }^{[10 a]}$ amines ${ }^{[10 b]}$ and electron-rich arenes. ${ }^{[9]}$ There is only one report on the use of indole iodonium salts involving a transition metal: the coppercatalyzed de-aromatization of indoles reported by You and coworkers. ${ }^{[11]}$ This stands in stark contrast with the hundreds of transformations reported for other aryl iodonium salts, ${ }^{[6]}$ and is probably due to the lower stability of indole and pyrrole iodonium salts in presence of metals and/or at higher temperature.
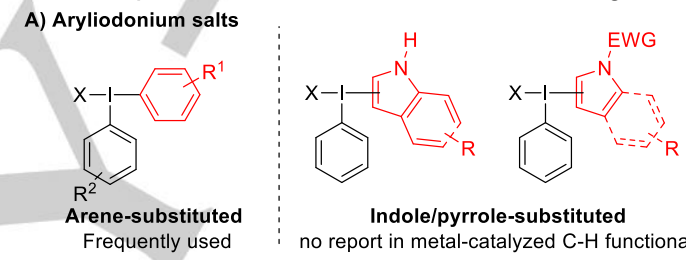

Frequently used

B) Reported benziodoxolones

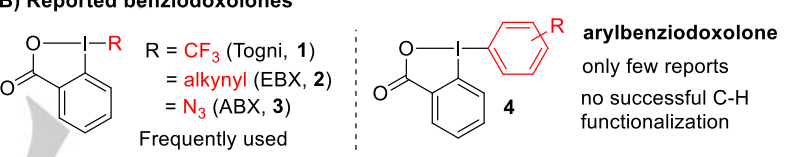

C) This Work: Indole/Pyrrole-BX and their use in C-H functionalization

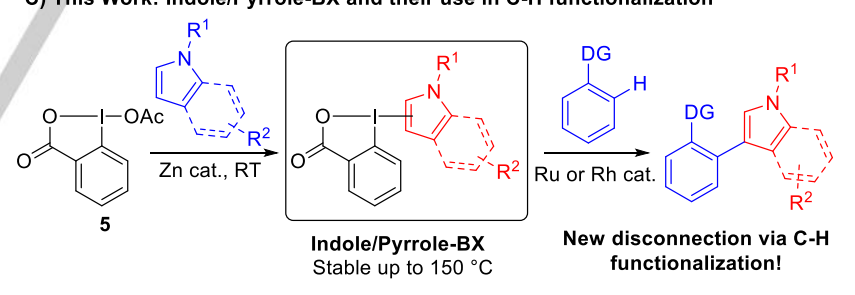

Scheme 1. Aryliodonium salts, benziodoxolones and Indole/Pyrrole-BX reagents.

The enhanced stability of cyclic hypervalent iodine reagents, in particular those obtained from 2-iodobenzoic acid and its derivatives - the benzidoxol(on)es - has been established since several decades (Scheme 1B). ${ }^{[12]}$ The use of reagents such as benziodoxolones 1-3 in group-transfer reactions (trifluoromethylation, alkynylation and azidation) has been investigated only more recently. ${ }^{[13]}$ In the short time since their introduction, they have already had a strong impact in the fields of synthetic and medicinal chemistry. Aryl benziodoxolones 4 have also displayed enhanced stability and are easily accessible, but they have not found broad application in synthesis. ${ }^{[14]}$ Indeed, in most reactions with nucleophiles, the benzoic acid group is transferred, limiting the scope of those transformations. ${ }^{[14 c, 15]}$ There is no report on indole or pyrrole based benziodoxol(on)e 
reagents, however, and the strongly acidic or oxidizing methods used for the introduction of other aryl groups cannot be used for these more sensitive heterocycles.

Herein, we report the first synthesis of pyrrole- and indolebased benziodoxolone reagents in one step from the heterocycles, based on a mild zinc-catalyzed direct iodonium transfer from acetoxy benziodoxolone (5) (Scheme 1C). The new reagents are air- moisture- and thermally stable. They were applied in the directed ortho $\mathrm{C}-\mathrm{H}$ functionalization of arenes using rhodium and ruthenium catalysts, resulting in bond formations that could not be achieved using reported methods and reagents.

To access the targeted Indole- and Pyrrole-BX reagents, we first applied recently reported methods for aryl benziodoxolone synthesis based on the oxidation of iodobenzoic acid (6) followed by reaction with $\mathrm{N}$-methyl indole (8a) in presence of triflic or sulfuric acid (Table 1, entries 1-2). ${ }^{[14]}$ However, only decomposition of the indole was observed. To avoid the use of oxidants, iodine(III) precursors were then examined. Starting from hydroxy benziodoxolone $\mathbf{7}$ with trimethylsilyl triflate (TMSOTf) as activating agent, ${ }^{[16]}$ decomposition was still occurring (entry 3 ). If a lower amount of TMSOTf was used, no conversion was observed (entry 4). A promising result was obtained with acetoxy benziodoxolone 5 as precursor: the desired indole-BX could be obtained in 16\% yield using 20 mol\% of TMSOTf (entries 5 and 6). ${ }^{[17]}$ This compound could be purified by silica gel column chromatography and was thermally stable up to $150{ }^{\circ} \mathrm{C} \cdot{ }^{[18]}$ No conversion was observed in absence of TMSOTf (entry 7 ). Better results were obtained with $\mathrm{Zn}(\mathrm{OTf})_{2}$ and $\mathrm{Cu}(\mathrm{OTf})_{2}$ as Lewis acids (entries 8 and 9), giving $9 a$ in $36 \%$ yield. Changing to dichloromethane as solvent with $\mathrm{Zn}(\mathrm{OTf})_{2}$ as catalyst, 9a was obtained in $97 \%$ yield with complete C3 selectivity (entry 10 ). This synthesis could be easily scaled up to give $9 \mathrm{a}$ in $87 \%$ yield on the $10 \mathrm{mmol}$ scale (entry 11).

Table 1. Optimization of the synthesis of Indole-BX 9a.

\begin{tabular}{|c|c|c|c|}
\hline Entry & lodine precursor & Reaction Conditions ${ }^{[a]}$ & Yield $^{[b]}(\%)$ \\
\hline 1 & 6 & $m \mathrm{CPBA}, \mathrm{TfOH}, \mathrm{CH}_{2} \mathrm{Cl}_{2}$, then $\mathrm{NH}_{3}^{[14 b]}$ & $<5^{[c]}$ \\
\hline 2 & 6 & Oxone, $\mathrm{H}_{2} \mathrm{SO}_{4}, \mathrm{CH}_{2} \mathrm{Cl}_{2}$, then $\mathrm{NaHCO}_{3}{ }^{[14 \mathrm{c}]}$ & $<5^{[c]}$ \\
\hline 3 & 7 & 1 equiv TMSOTf, $\mathrm{CH}_{3} \mathrm{CN}$, then pyridine & $<5^{[c]}$ \\
\hline 4 & 7 & $20 \mathrm{~mol} \%$ TMSOTf, $\mathrm{CH}_{3} \mathrm{CN}$ & $<5^{[\mathrm{d}]}$ \\
\hline 5 & 5 & 1 equiv TMSOTf, $\mathrm{Et}_{2} \mathrm{O}$ & $<5^{[c]}$ \\
\hline 6 & 5 & $20 \mathrm{~mol} \%$ TMSOTf, $\mathrm{Et}_{2} \mathrm{O}$ & 16 \\
\hline 7 & 5 & $\mathrm{Et}_{2} \mathrm{O}$ & $<5^{[\mathrm{d}]}$ \\
\hline 8 & 5 & $20 \mathrm{~mol} \% \mathrm{Zn}(\mathrm{OTf})_{2}, \mathrm{Et}_{2} \mathrm{O}$ & 36 \\
\hline 9 & 5 & $20 \mathrm{~mol} \% \mathrm{Cu}(\mathrm{OTf})_{2}, \mathrm{Et}_{2} \mathrm{O}$ & $36^{[\mathrm{cc}]}$ \\
\hline 10 & 5 & $20 \mathrm{~mol} \% \mathrm{Zn}(\mathrm{OTf})_{2}, \mathrm{CH}_{2} \mathrm{Cl}_{2}$ & 97 \\
\hline 11 & 5 & $20 \mathrm{~mol} \% \mathrm{Zn}(\mathrm{OTf})_{2}, \mathrm{CH}_{2} \mathrm{Cl}_{2}$ & $87^{[e]}$ \\
\hline
\end{tabular}

[a] Reactions were performed on $0.10 \mathrm{mmol}$ scale with 1.1 equiv of iodine precursor. [b] Isolated yield after purification by column chromatography. [c] Decomposition of $\mathrm{N}$-methylindole (8a) was observed. [d] No conversion of $\mathbf{7}$ or 8a. [e] Reaction performed on $10 \mathrm{mmol}$ scale.
Free $\mathrm{NH}$ IndoleBX 9b could be also synthesized in $78 \%$ yield starting from $\mathrm{N}$-silylated indole (Figure 1). The hypervalent structure of this compound was confirmed by X-ray analysis. ${ }^{[18]}$ Different alkyl groups on the nitrogen were well tolerated (9c-e). Reagent 9 f bearing a C2-substituted indole was also obtained in good yield, but no conversion was observed with C3-substitution. Arenes bearing ethers $(\mathbf{9 g})$, halogens $(\mathbf{9} \mathbf{h} \mathbf{j})$ and a sensitive boronic ester (9k) could also be used. Reagent 9 I derived from a less reactive azaindole was still isolated in $30 \%$ yield. PyrroleBX $9 \mathrm{~m}-\mathrm{q}$ were also successfully synthesized. When starting from $\mathrm{N}$ silylated pyrrole, a complete C3 regioselectivity was achieved $(9 \mathrm{~m})$. When methyl-and benzyl-pyrrole where used as substrates, mixtures of separable $\mathrm{C} 2: \mathrm{C} 3$ functionalized reagents were obtained. The availability of both isomers in pure form opened the way for the selective synthesis of $\mathrm{C2}$ - or C3- functionalized pyrroles by reaction with nucleophiles. The synthesis of new pyrrole and indole electrophilic synthons is especially important, as none of the currently available reagents can be used in $\mathrm{C}-\mathrm{H}$ functionalization reactions, in contrast to other classes of heterocycles. Nevertheless, the use of other electron-rich aromatic compounds could also be envisaged. Indeed, carbazoleBX 9r, thiophene-BX 9s and furan-BX 9t were also obtained in low to moderate yields without further optimization.

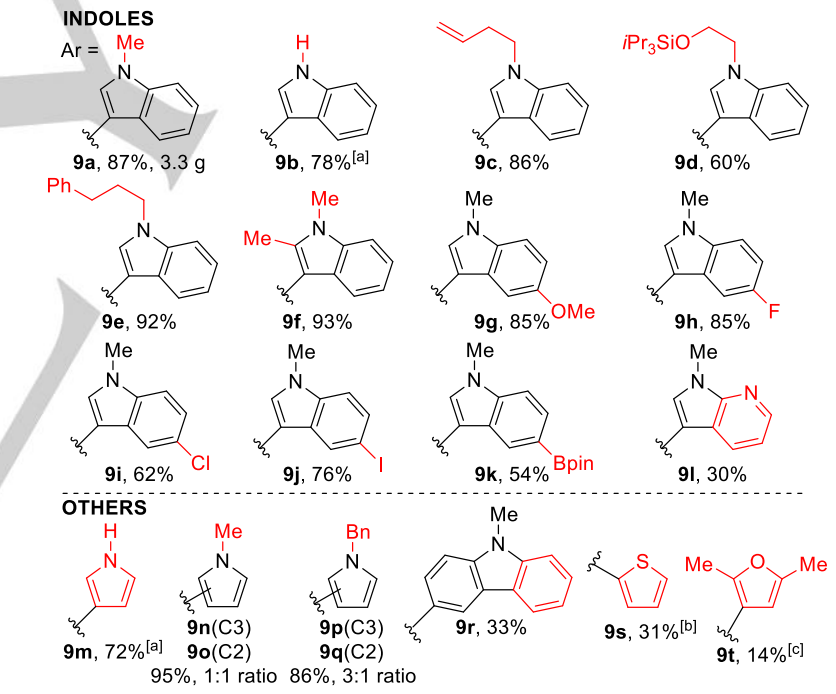

Figure 1. Scope of heteroaromatic benziodoxole reagents. Reaction conditions $1.00 \mathrm{mmol}$ heterocycle, $1.10 \mathrm{mmol} 5,0.200 \mathrm{mmol} \mathrm{Zn}(\mathrm{OTf})_{2}, 0.05 \mathrm{M}$ in DCM, RT, open air. Isolated yields. [a] $\mathrm{Sc}(\mathrm{OTf})_{3}$ was used starting from $\mathrm{N}$-TBS heterocycle. [b] $\mathrm{Sc}(\mathrm{OTf})_{3}$ was used. [c] $\ln (\mathrm{OTf})_{3}$ was used.

Preliminary investigations showed that the reactivity of indole-BX 9a differed from the one of both aryliodonium salts and EBX reagents (Scheme 2). The reaction of EBX reagents and aryliodonium salts with thiols, alcohols and stabilized carbon nucleophiles in presence of base is well established, ${ }^{[19]}$ but no reaction occurred with $\mathbf{9 a}$. In the case of thiol 10, a moderate yield of thiol indolation product $\mathbf{1 1}$ could be obtained in presence of a copper catalyst (Scheme 2A). Palladium-catalyzed $\mathrm{C}-\mathrm{H}$ bond arylation, ${ }^{[6 a, 20]}$ was also unsuccessful. In contrast, highly efficient $\mathrm{C}-\mathrm{H}$ functionalizations could be developed using either rhodium- 
or ruthenium catalysts. ${ }^{[21]}$ Importantly, complete and selective transfer of the indole was obtained to give $13 \mathbf{a}$ and $15 a$, with no coupling of benzoic acid.

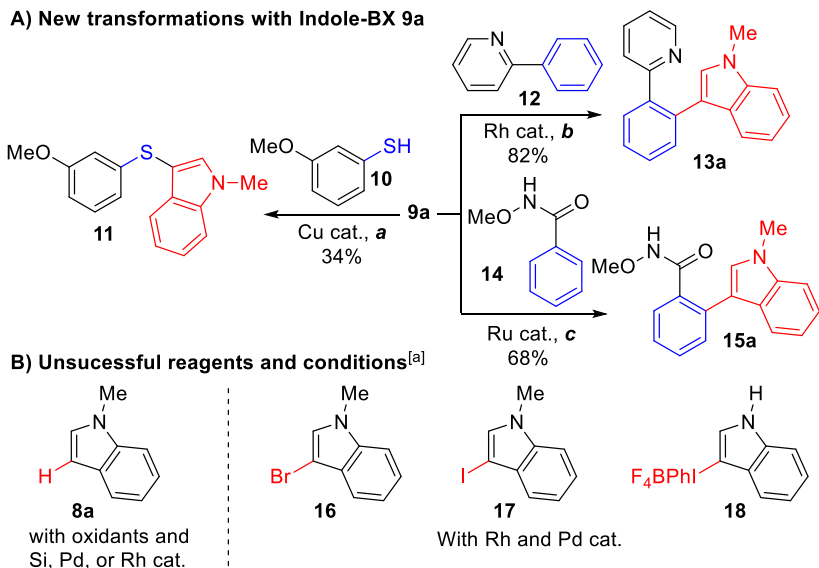

Scheme 2. Preliminary studies of the reactivity of Indole-BX 9a. Reaction conditions: a) 10, $20 \mathrm{~mol} \% \mathrm{CuOTf} \cdot \mathrm{C}_{6} \mathrm{H}_{6}, \mathrm{CH}_{2} \mathrm{Cl}_{2}, \mathrm{RT}$; b) $12,2.5 \mathrm{~mol} \%$ $\left[\mathrm{Rh}\left(\mathrm{Cp}^{*} \mathrm{Cl}_{2}\right)\right]_{2}, 10 \mathrm{~mol} \%$ NaOPiv, $10 \mathrm{~mol} \% \mathrm{AgSbF}_{6}$, DCE, $0.1 \mathrm{M}, 50{ }^{\circ} \mathrm{C}$; c) 14 $10 \mathrm{~mol} \% \mathrm{Ru}\left[p\right.$-cymene $\left.(\mathrm{AdCOO})_{2}\right],{ }^{[22]}$ trifluoroethanol, $0.1 \mathrm{M}, 60{ }^{\circ} \mathrm{C}$. [a] See supporting Information for detailed reaction conditions.

The latter results are especially interesting, as the introduction of an indole on a benzene $\mathrm{C}-\mathrm{H}$ bond in ortho position to a pyridine or a benzamide has never been reported. This is surprising, as ortho $\mathrm{C}-\mathrm{H}$ bond functionalization in arenes is now considered as a mature field. ${ }^{[23]}$ When considering the high importance of indole and pyrrole heterocycles in synthetic and medicinal chemistry, realizing such a transformation would be an important breakthrough in the field. We wondered therefore if established methods for the introduction of indoles onto other types of $\mathrm{C}-\mathrm{H}$ bonds could be used to access products $13 \mathbf{a}$ and $15 \mathbf{a}$. However, when reported methods for $\mathrm{C}-\mathrm{H}$ indolation based on oxidative $\mathrm{C}$ $\mathrm{H} / \mathrm{C}-\mathrm{H}$ couplings ${ }^{[24-26]}$ with indole $8 \mathrm{a}$ or $\mathrm{C}-\mathrm{H}$ functionalization with electrophilic indoles 16,17 and $18,{ }^{[18]}$ were examined, compounds 13a and 15a could not be obtained (Scheme 2B). Therefore, the discovery of Indole-BX reagents allowed new $\mathrm{C}-\mathrm{H}$ functionalizations which were not possible before.

The scope of the rhodium-catalyzed $\mathrm{C}-\mathrm{H}$ functionalization was then examined (Scheme $3 \mathrm{~A}$ ). Indoles $\mathbf{1 3 b}$ and $\mathbf{1 3 c}$ bearing either a free $\mathrm{NH}$ group or an alkyl chain were obtained in good yield. Ethers, halogens and a sensitive boronic ester were well tolerated on the benzene ring of the indole reagent (13d-h). The regioselective synthesis of both $\mathrm{C} 2$ and $\mathrm{C} 3$ substituted pyrroles was possible (13i-m) ${ }^{[27]}$ Methoxy, halogens, esters and cyanides functional groups could be present on the arene substrate (13nr). Diarylation was also possible using an excess of reagent $\mathbf{8 a}$ to give product 19. Good results were also obtained with other heterocyclic directing groups such as pyrimidine $(20)$ or pyrazole (21). Benzoquinoline 22 and pyrimidine-substituted indole 23 were also formed in good yield. When quinoline $\mathrm{N}$-oxide was used as the starting material, quinolone $\mathbf{2 4}$ was obtained in $38 \%$ yield. The developed $\mathrm{C}-\mathrm{H}$ arylation could also be applied to a purinebased nucleoside to give product 25 .

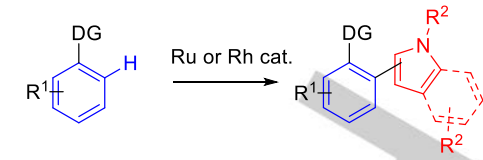

A) With $2.5 \mathrm{~mol} \%\left[\mathrm{Rh}\left(\mathrm{Cp}^{*} \mathrm{Cl}_{2}\right)\right]_{2}, 10 \mathrm{~mol} \%$ NaOPiv, $10 \mathrm{~mol}^{2} \mathrm{AgSbF}_{6}, \mathrm{DCE}, 0.1 \mathrm{M}, 50{ }^{\circ} \mathrm{C}$
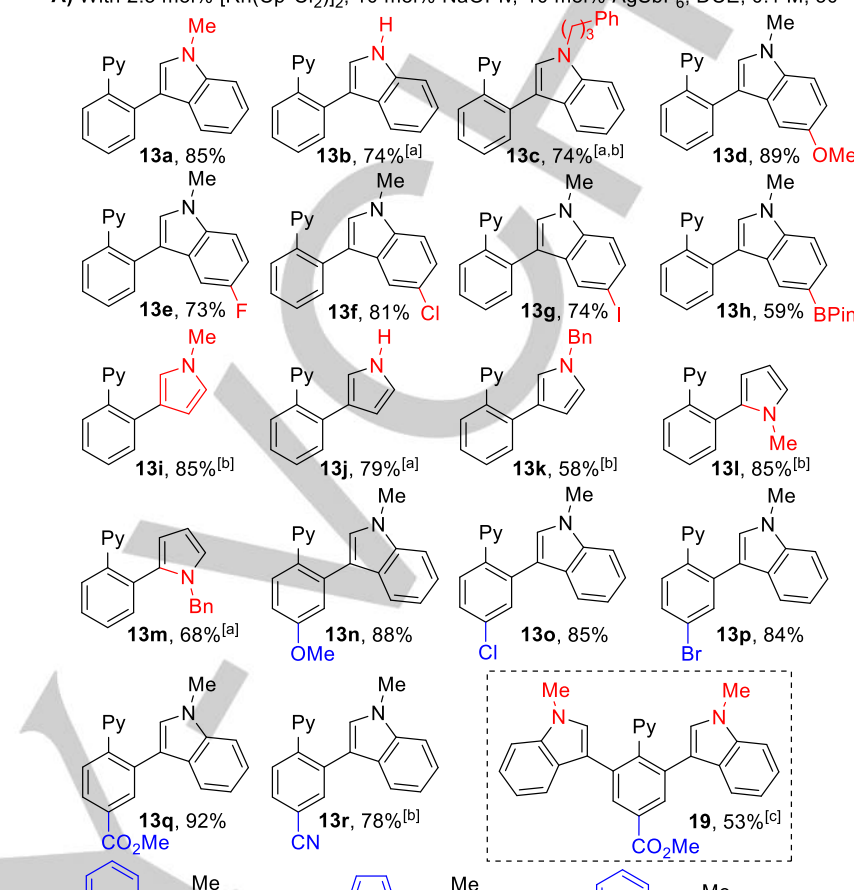

$\mathrm{Cl} 130,85 \% \quad \mathrm{Br}_{13 \mathrm{p}, 84 \%}$
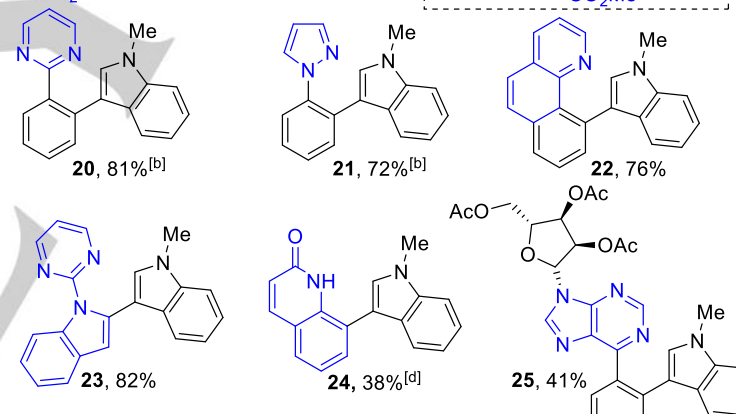

$\overbrace{-O A C}^{O A C}$

B) $10 \mathrm{~mol} \% \mathrm{Ru}[p \text {-cymene(AdCOO) })_{2}$, trifluoroethanol, $0.1 \mathrm{M}, 60^{\circ} \mathrm{C}$
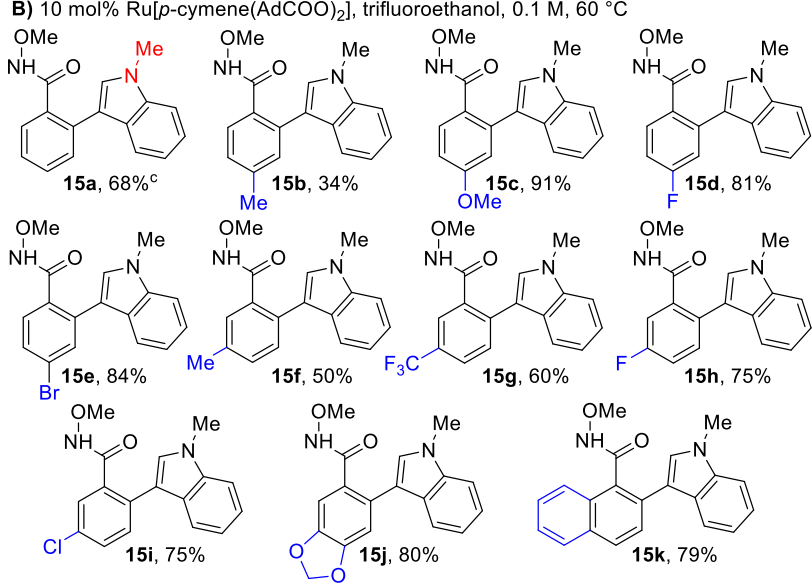

Scheme 3. Scope of the Rh- and Ru- catalyzed $\mathrm{C}-\mathrm{H}$ Functionalizations. Reactions were performed on $0.30 \mathrm{mmol}$ scale. [a] 0.1 M in DCE:MeOH (1:1 ratio). [b] at $80^{\circ} \mathrm{C}$. [c] Using $0.660 \mathrm{mmol} 8 \mathrm{a}$. [d] at $100^{\circ} \mathrm{C}$. 
The thermal stability of the developed reagents was essential to achieve a broad scope, as several substrates required higher temperature to reach useful conversion $\left(80^{\circ} \mathrm{C}\right.$ for products 13c,k,l,r, 20 and 21 , and $100^{\circ} \mathrm{C}$ for compound 24). Preliminary investigations of the scope of the ruthenium-catalyzed $\mathrm{C}-\mathrm{H}$ functionalization were then conducted (Scheme 3B). The benzamide directing group is attractive, as it can be easily modified or removed. Alkyl groups, ethers, trifluoromethyl groups and halogens were all well tolerated both in para and meta position to the amide (15a-i). Tetrasubstituted arene $\mathbf{1 5} \mathbf{j}$ and naphthyl derivatives $\mathbf{1 5 k}$ were also obtained in good yields.

In conclusion, we have reported the first synthesis of indole- and pyrrole substituted benziodoxolone reagents. Indole- and Pyrrole$B X$ were synthesized in one-step from the heterocycles under mild Lewis acid catalyzed conditions and are thermally stable up to $150{ }^{\circ} \mathrm{C}$. These new reagents can be used for the Rh- and Ruortho $\mathrm{C}-\mathrm{H}$ functionalization of arenes using heterocyclic and benzamide directing groups, a transformation that could not be realized using previously reported methods. Many of the obtained products are new combinations of privileged (hetero)arenes, covering interesting chemical space for medicinal chemistry. The availability of stable electrophilic indole and pyrrole synthons is expected to lead to broad applications in synthetic and medicinal chemistry.

\section{Acknowledgements}

This work is supported by the Swiss National Science Foundation (No. 200021_159920), the European Research Council (ERC Starting Grant 334840) and the COST action CA15106 (C-H Activation in Organic Synthesis, CHAOS). We thank $\mathrm{Dr} \mathrm{R}$. Scopelliti and Dr F. F. Tirani from ISIC at EPFL for X-ray analysis, and Dr. Fides Benfatti and Ms. Marylene Stempien from Syngenta Crop Science for DSC experiments. Mr. Elliott Le Du from EPFL is thanked for performing the thiol indolation experiment.

\section{Keywords: Indoles $\cdot$ Hypervalent lodine $\cdot$ Umpolung $\cdot \mathrm{C}-\mathrm{H}$} Functionalization $\cdot$ Heterocycles

[1] G. W. Gribble, Indole Ring Synthesis: From Natural Products to Drug Discovery. Wiley: 2016.

[2] G. R. Humphrey, J. T. Kuethe, Chem. Rev. 2006, 106, 2875.

[3] M. Bandini, Org. Biomol. Chem. 2013, 11, 5206.

[4] Selected example: G. Li, E. Wang, H. Chen, H. Li, Y. Liu, P. G. Wang, Tetrahedron 2008, 64, 9033.

[5] A. Yoshimura, V. V. Zhdankin, Chem. Rev. 2016, 116, 3328.

[6] a) E. A. Merritt, B. Olofsson, Angew. Chem., Int. Ed. 2009, 48, 9052. b) J. Malmgren, S. Santoro, N. Jalalian, F. Himo, B. Olofsson, Chem. Eur. J. 2013, 19, 10334.

[7] a) B. Y. Karele, L. E. Treigute, S. V. Kalnin, I. P. Grinberga, O. Y. Neiland, Chem. Heterocycl. Compd. 1974, 10, 189. b) V. A. Budylin, M. S. Ermolenko, F. A. Chugtai, P. A. Sharbatyan, A. N. Kost, Chem. Heterocycl. Compd. 1981, 17, 1095. c) R. M. Moriarty, Y. Y. Ku, M. Sultana, A. Tuncay, Tetrahedron Lett. 1987, 28,3071 .
[8] K. Ishida, H. Togo, K. Moriyama, Chem. Asian J. 2016, 11, 3583

[9] K. Morimoto, Y. Ohnishi, D. Koseki, A. Nakamura, T. Dohi, Y. Kita, Org Biomol. Chem. 2016, 14, 8947.

[10] a) D. Lubriks, I. Sokolovs, E. Suna, J. Am. Chem. Soc. 2012, 134, 15436; b) I. Sokolovs, D. Lubriks, E. Suna, J. Am. Chem. Soc. 2014, 136, 6920.

[11] C. Liu, J.-C. Yi, X.-W. Liang, R.-Q. Xu, L.-X. Dai, S.-L. You, Chem. Eur. J. 2016, 22, 10813.

[12] V. V. Zhdankin, Curr. Org. Synth. 2005, 2, 121.

[13] a) J. Charpentier, N. Frueh, A. Togni, Chem. Rev 2015, 115, 650; b) Y. Li, D. P. Hari, M. V. Vita, J. Waser, Angew. Chem., Int. Ed. 2016, 55, 4436.

[14] a) F. M. Beringer, I. Lillien, J. Am. Chem. Soc. 1960, 82, 725; b) E. A. Merritt, B. Olofsson, Eur. J. Org. Chem. 2011, 3690; c) M. S. Yusubov, R. Y. Yusubova, V. N. Nemykin, V. V. Zhdankin, J. Org. Chem. 2013, 78, 3767.

[15] Selected examples: a) R. A. Scherrer, H. R. Beatty, J. Org. Chem 1980, 45, 2127 ; b) M. Xia, Z. C. Chen, Synth. Commun. 2000, 30, 63; c) M. W. Justik, J. D. Protasiewicz, J. B. Updegraff, Tetrahedron Lett. 2009, 50, 6072; d) J. Carstens, M. R. Heinrich, W. Steglich, Tetrahedron Lett. 2013, 54, 5445.

[16] V. V. Zhdankin, C. J. Kuehl, A. P. Krasutsky, J. T. Bolz, A. J. Simonsen, J. Org. Chem. 1996, 61, 6547

[17] Diethyl ether was used as solvent due to the low solubility of 5 in acetonitrile. [18] See Supporting Information.

[19] Selected examples: a) R. Frei, M. D. Wodrich, D. P. Hari, P. A. Borin, C. Chauvier, J. Waser, J. Am. Chem. Soc. 2014, 136, 16563; b) R. Ghosh, E. Lindstedt, N. Jalalian, B. Olofsson, ChemistryOpen 2014, 3, 54; c) D. Fernandez Gonzalez, J. P. Brand, J. Waser, Chem. Eur. J. 2010, 16, 9457.

[20] Selected examples: a) D. Kalyani, N. R. Deprez, L. V. Desai, M. S. Sanford, J. Am. Chem. Soc. 2005, 127, 7330; b) O. Daugulis, V. G. Zaitsev, Angew. Chem., Int. Ed. 2005, 44, 4046.

[21] Selected examples: a) C. Feng, T.-P. Loh, Angew. Chem., Int. Ed. 2014, 53, 2722; b) F. Xie, Z. Qi, S. Yu, X. Li, J. Am. Chem. Soc. 2014, 136, 4780; c) K. D. Collins, F. Lied, F. Glorius, Chem. Commun. 2014, 50, 4459; d) R. Boobalan, P. Gandeepan, C.-H. Cheng, Org. Lett. 2016, 18, 3314. Nevertheless, extensive optimization of the reaction conditions was still needed to obtain good yields with Indole-BX 9a, see the Supporting Information for details.

[22] Z. Ruan, S.-K. Zhang, C. Zhu, P. N. Ruth, D. Stalke, L. Ackermann, Angew. Chem., Int. Ed. 2017, 56, 2045.

[23] J. Q. Yu, in Topics in Current Chemistry: C-H Activation; Springer, 2010; Vol. 292.

[24] Review: a) Y. Yang, J. Lan, J. You, Chem. Rev. 2017, 117, 8787; Selected examples of C-H indolation: b) D. R. Stuart, K. Fagnou, Science 2007, 316 1172; c) D. R. Stuart, E. Villemure, K. Fagnou, J. Am. Chem. Soc. 2007, 129, 12072; d) B. J. Li, S. L. Tian, Z. Fang, Z. J. Shi, Angew. Chem., Int. Ed. 2008, 47, 1115; e) Y. Kita, K. Morimoto, M. Ito, C. Ogawa, A. Goto, T. Dohi, J. Am. Chem. Soc. 2009, 131, 1668; f) J. Wencel-Delord, C. Nimphius, H. Wang, F. Glorius, Angew. Chem., Int. Ed. 2012, 51, 13001.

[25] Selected examples for coupling of indoles with fluorinated arenes: a) C. Y. $\mathrm{He}$, Q. Q. Min, X. G. Zhang, Organometallics 2012, 31, 1335; b) X. C. Cambeiro, N. Ahlsten, I. Larrosa, J. Am. Chem. Soc. 2015, 137, 15636.

[26] Selected examples for coupling of indoles with electron-poor heterocycles: a) X. Gong, G. Y. Song, H. Zhang, X. W. Li, Org. Lett. 2011, 13, 1766; b) M. Nishino, K. Hirano, T. Satoh, M. Miura, Angew. Chem., Int. Ed. 2012, 51, 6993; c) N. Kuhl, M. N. Hopkinson, F. Glorius, Angew. Chem., Int. Ed. 2012, 51, 8230; d) Z. Wang, F. J. Song, Y. S. Zhao, Y. M. Huang, L. Yang, D. B. Zhao, J. B. Lan, J. S. You, Chem. Eur. J. 2012, 18, 16616; e) X. R. Qin, H. Liu, D. K. Qin, Q. Wu J. S. You, D. B. Zhao, Q. Guo, X. L. Huang, J. B. Lan, Chem. Sci. 2013, 4, 1964 f) B. Liu, Y. M. Huang, J. B. Lan, F. J. Song, J. S. You, Chem. Sci. 2013, 4 $2163 ;$ g) K. Morimoto, K. Sakamoto, T. Ohshika, T. Dohi, Y. Kita, Angew. Chem., Int. Ed. 2016, 55, 3652

[27] The rhodium-catalyzed $\mathrm{C}-\mathrm{H}$ functionalization was not successful with reagents 9 r-t. 


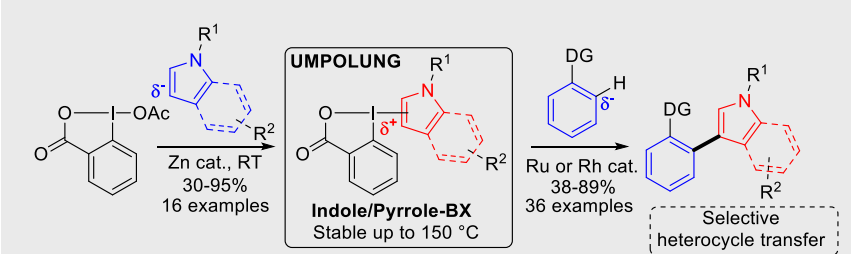

Electrophilic Indoles/Pyrroles: The first synthesis of indole and pyrrole-derived benziodoxole reagents in one step from the heterocycles is reported. The new Indoleand Pyrrole- BX reagents are stable up to $150{ }^{\circ} \mathrm{C}$ and can be used for selective heterocycle transfer onto the $\mathrm{C}-\mathrm{H}$ bonds of arenes ortho to directing groups by using rhodium or ruthenium catalysts.
Paola Caramenti, Stefano Nicolai, Jerome Waser*

Page No. - Page No.

Indole- and Pyrrole-BX: Bench-Stable Hypervalent lodine Reagent for Heterocycle Umpolung 


\section{Supporting Information}

\section{Table of contents}

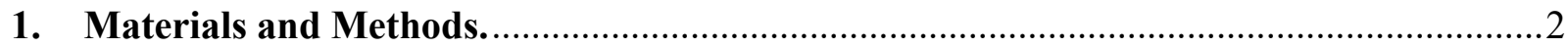

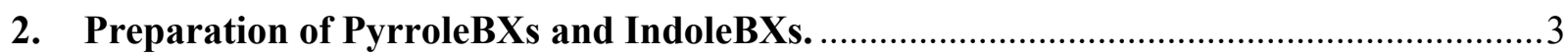

2.1 Preparation of Starting Materials for PyrroleBXs and IndoleBXs. ...............................

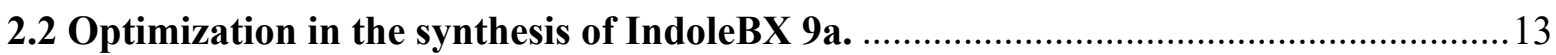

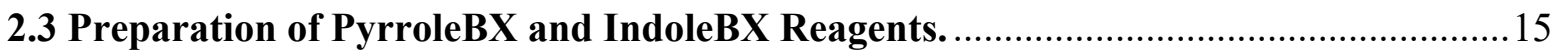

3.2 Procedure for Thio-indolization via Lewis-acid activated IndoleBX ...........................34

3.3 Optimization of the Rh-Catalyzed Indolization of Arenes via $\mathrm{C}-\mathrm{H}$ activation..............35

3.4 Control experiments for the Indolization of Arenes via C-H activation......................38

3.5 Scope of the Rh-Catalyzed Indolization via C-H activation......................................42

4. Ru-Catalyzed C-H Indolization of Arenes via $\mathrm{C}-\mathrm{H}$ activation........................................58

4.1 Preparation of starting materials for Ru-Catalyzed $\mathrm{C}-\mathrm{H}$ activation...........................58

4.2 Optimization of the Ru-Catalyzed Indolization of Arenes via C-H activation.............64

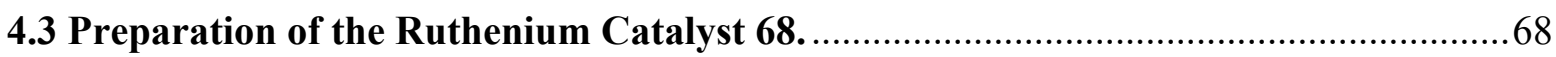

4.4 Scope of the Ru-Catalyzed Indolization via C-H activation......................................69

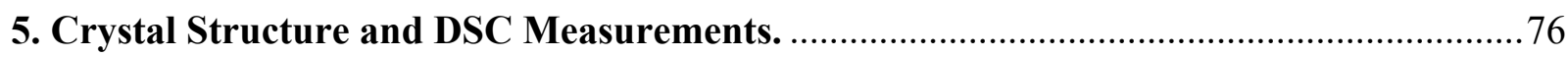

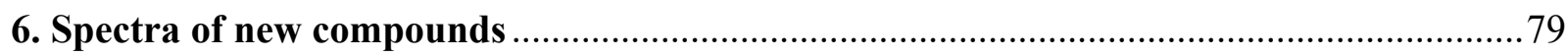




\section{Materials and Methods.}

All reactions were carried out in oven dried glassware under an atmosphere of nitrogen, unless stated otherwise. For quantitative flash chromatography, technical grade solvents were used. For flash chromatography for analysis, HPLC grade solvents from Sigma-Aldrich were used. THF, $\mathrm{Et}_{2} \mathrm{O}, \mathrm{CH}_{3} \mathrm{CN}$, toluene, hexane and $\mathrm{CH}_{2} \mathrm{Cl}_{2}$ were dried by passage over activated alumina under nitrogen atmosphere $\left(\mathrm{H}_{2} \mathrm{O}\right.$ content $<10 \mathrm{ppm}$, Karl-Fischer titration $)$. The solvents were degassed through Freeze-Pump-Thaw method when mentioned. All chemicals were purchased from Acros, Aldrich, Fluka, VWR, Aplichem, or Merck and used as such unless otherwise stated. Chromatographic purification was performed as flash chromatography using Macherey-Nagel silica 40-63, $60 \AA$, with the solvents indicated as eluent under 0.1-0.5 bar pressure. TLC was performed on Merck silica gel $60 \mathrm{~F}_{254}$ TLC glass plates or aluminium plates and visualized with UV light, permanganate stain, CAN stain, or Anisaldehyde stain. Melting points were measured on a Büchi B-540 melting point apparatus using open glass capillaries, the data is uncorrected. ${ }^{1} \mathrm{H}$ NMR spectra were recorded on a Brucker DPX-400 $400 \mathrm{MHz}$ spectrometer in $\mathrm{CDCl}_{3}, \mathrm{DMSO}-d_{6}$ $\mathrm{CD}_{3} \mathrm{OD}, \mathrm{C}_{6} \mathrm{D}_{6}$ and $\mathrm{CD}_{2} \mathrm{Cl}_{2}$, all signals are reported in ppm with the internal chloroform signal at $7.26 \mathrm{ppm}$, the internal DMSO signal at $2.50 \mathrm{ppm}$ the internal methanol signal at $3.30 \mathrm{ppm}$, the internal dichloromethane signal at $5.30 \mathrm{ppm}$ as standard. The data is being reported as $(\mathrm{s}=$ singlet, $\mathrm{d}=$ doublet, $\mathrm{t}=$ triplet, $\mathrm{q}=$ quadruplet, $\mathrm{qi}=$ quintet, $\mathrm{m}=$ multiplet or unresolved, $\mathrm{br}=$ broad signal, app = apparent, coupling constant(s) in $\mathrm{Hz}$, integration, interpretation). ${ }^{13} \mathrm{C}-\mathrm{NMR}$ spectra were recorded with ${ }^{1} \mathrm{H}$-decoupling on a Brucker DPX-400 $100 \mathrm{MHz}$ spectrometer in $\mathrm{CDCl}_{3}$, DMSO- $d_{6}$, $\mathrm{CD}_{3} \mathrm{OD}$ or $\mathrm{CD}_{2} \mathrm{Cl}_{2}$, all signals are reported in ppm with the internal chloroform signal at $77.0 \mathrm{ppm}$, the internal DMSO signal at $39.5 \mathrm{ppm}$, the internal methanol signal at $49.0 \mathrm{ppm}$ and the internal dichloromethane signal at $54.0 \mathrm{ppm}$ as standard. Infrared spectra were recorded on a JASCO FTIR B4100 spectrophotometer with an ATR PRO410-S and a ZnSe prisma and are reported as $\mathrm{cm}^{-1}$ ( $\mathrm{w}=$ weak, $\mathrm{m}=$ medium, $\mathrm{s}=$ strong, $\mathrm{br}=$ broad). High resolution mass spectrometric measurements were performed by the mass spectrometry service of ISIC at the EPFL on a MICROMASS (ESI) Q-TOF Ultima API. 


\section{Preparation of PyrroleBXs and IndoleBXs.}

The synthesis of the precursors for HeterocyclicBX reagents (7 and 5) 9a-9r had been already described before. ${ }^{1,2}$ The procedures here reported are taken from the cited publications to facilitate reproduction of the results by having all the data in the same file.

\subsection{Preparation of Starting Materials for PyrroleBXs and IndoleBXs.}

1-Hydroxy-1,2-benziodoxol-3-(1H)-one (27)

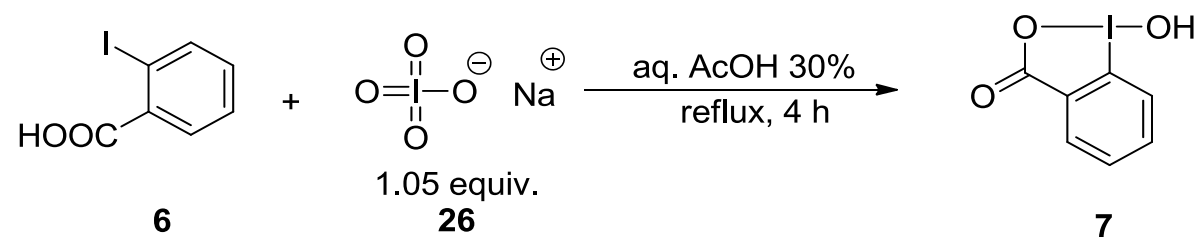

Following a reported procedure, $\mathrm{NaIO}_{4} \mathbf{2 6}(7.24 \mathrm{~g}, 33.8 \mathrm{mmol}, 1.05$ equiv) and 2-iodobenzoic acid 6 (8.00 g, $32.2 \mathrm{mmol}, 1.00$ equiv) were suspended in 30\% (v/v) aq. AcOH (48 mL). The mixture was vigorously stirred and refluxed for $4 \mathrm{~h}$. The reaction mixture was then diluted with cold water $(180 \mathrm{~mL})$ and allowed to cool to room temperature, protecting it from light. After $1 \mathrm{~h}$, the crude product was collected by filtration, washed on the filter with ice water $(3 \times 20 \mathrm{~mL})$ and acetone (3 x $20 \mathrm{~mL})$, and air-dried in the dark to give the pure product 7 ( $8.30 \mathrm{~g}, 31.0 \mathrm{mmol}, 98 \%$ yield $)$ as a colorless solid. ${ }^{1}$ H NMR (400 MHz, DMSO- $d_{6}$ ): $\delta 8.02$ (dd, $J=7.7,1.4 \mathrm{~Hz}, 1 \mathrm{H}, \mathrm{Ar} H$ ), 7.97 (m, $1 \mathrm{H}, \operatorname{Ar} H), 7.85(\mathrm{dd}, J=8.2,0.7 \mathrm{~Hz}, 1 \mathrm{H}, \operatorname{Ar} H), 7.71(\mathrm{td}, J=7.6,1.2 \mathrm{~Hz}, 1 \mathrm{H}, \operatorname{Ar} H) .{ }^{13} \mathbf{C}$ NMR $(100$ $\left.\mathrm{MHz},\left(\mathrm{CD}_{3}\right)_{2} \mathrm{SO}\right): \delta 167.7,134.5,131.5,131.1,130.4,126.3,120.4$. IR $v 3083(\mathrm{w}), 3060(\mathrm{w}), 2867$ (w), 2402 (w), 1601 (m), 1585 (m), 1564 (m), 1440 (m), 1338 (s), 1302 (m), 1148 (m), 1018 (w), $834(\mathrm{~m}), 798(\mathrm{w}), 740(\mathrm{~s}), 694(\mathrm{~s}), 674(\mathrm{~m}), 649(\mathrm{~m})$. NMR values are in accordance with the data reported in literature. ${ }^{1}$

1-Acetoxy-1,2-benziodoxol-3-(1H)-one (4)

[1] D. P. Hari, J. Waser, J. Am. Chem. Soc. 2016, 138, 2190-2193.

[2] G. L. Tolnai, S. Ganss, J. P. Brand, J. Waser, Org. Lett. 2013, 15, 112-115. 


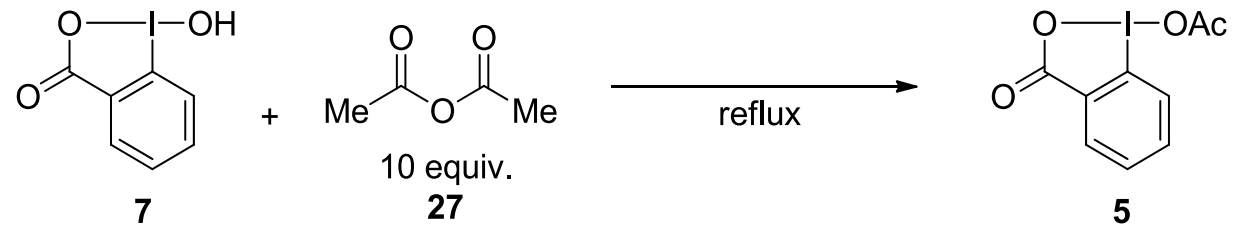

Following a reported procedure, ${ }^{3}$ 1-hydroxy-1,2-benziodoxol-3-(1H)-one 7, $10.3 \mathrm{~g}, 39.1 \mathrm{mmol}$, 1.00 equiv.) was suspended in acetic anhydride $27(35 \mathrm{~mL})$ and heated to reflux for 30 minutes. The resulting clear, slightly yellow solution was slowly let to warm up to room temperature and then cooled to $0{ }^{\circ} \mathrm{C}$ for 30 minutes. The white suspension was filtered and the filtrate was again cooled to $0{ }^{\circ} \mathrm{C}$ for 30 minutes. The suspension was once again filtered and the combined two batches of solid product were washed with hexane $(2 \times 20 \mathrm{~mL})$ and dried in vacuo to afford 1Acetoxy-1,2-benziodoxol-3-(1H)-one 5 (10.8 g, $35.3 \mathrm{mmol}, 90 \%)$ as a white solid. ${ }^{1} \mathbf{H}$ NMR $\left(\mathrm{CDCl}_{3}, 400 \mathrm{MHz}\right) \delta 8.24(\mathrm{dd}, J=7.6,1.6 \mathrm{~Hz}, 1 \mathrm{H}, \mathrm{Ar} H), 8.00(\mathrm{dd}, J=8.3,1.0 \mathrm{~Hz}, 1 \mathrm{H}, \mathrm{Ar} H), 7.92$ $(\mathrm{ddd}, J=8.4,7.2,1.6 \mathrm{~Hz}, 1 \mathrm{H}, \mathrm{Ar} H), 7.71(\mathrm{td}, J=7.3,1.1 \mathrm{~Hz}, 1 \mathrm{H}, \mathrm{ArH}), 2.25\left(\mathrm{~s}, 3 \mathrm{H}, \mathrm{COCH}_{3}\right) .{ }^{13} \mathrm{C}$ NMR $\left(\mathrm{CDCl}_{3}, 100 \mathrm{MHz}\right) \delta 176.5,168.2,136.2,133.3,131.4,129.4,129.1,118.4,20.4$. NMR values are in accordance with the data reported in literature. ${ }^{4}$

\section{General Procedure GP1 for the N-Methylation of Indoles.}

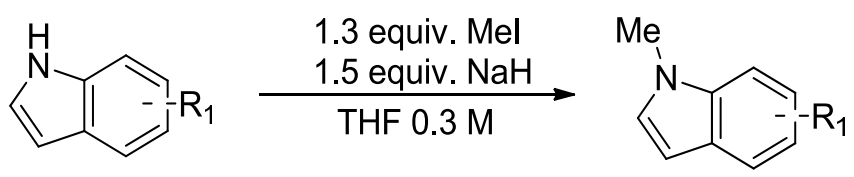

The corresponding indole (1.00 - $5.00 \mathrm{mmol}, 1.00$ equiv.) was dissolved in dry $\operatorname{THF}(0.3 \mathrm{M})$. Sodium hydride ( $60 \%$ suspension in mineral oil; 1.50 equiv.) was slowly added under $\mathrm{N}_{2}$ flow at 0 ${ }^{\circ} \mathrm{C}$. After being stirred at $0{ }^{\circ} \mathrm{C}$ for 15 min,the reaction mixture was allowed to warm to r.t for 1.5 h. It was then cooled back to $0{ }^{\circ} \mathrm{C}$ and methyl iodide (1.30 equiv.) was added. The mixture was warmed to r.t. and stirred overnight. After cooling again to $0^{\circ} \mathrm{C}$, the reaction was quenched with water $(10 \mathrm{~mL})$, extracted with $\mathrm{Et}_{2} \mathrm{O}(3 \times 10 \mathrm{~mL})$, the combined organic layers were dried over $\mathrm{MgSO}_{4}$, and the solvent removed under reduced pressure. The resulting crude product was purified via flash column chromatography (Pentane:EtOAc 9:1-4:1), to give the desired N-methylated indole.

[3] A. T. Parsons, S. L. Buchwald, Angew. Chem. Int. Ed. 2011, 50, 9120-9123

[4] P. Eisenberger, S. Gischig, A. Togni, Chem. - Eur. J. 2006, 12, 2579-2586. 


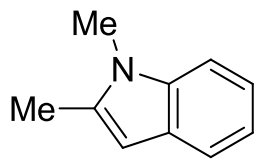

28

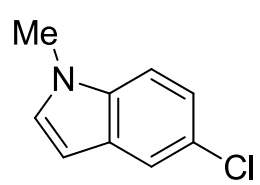

31

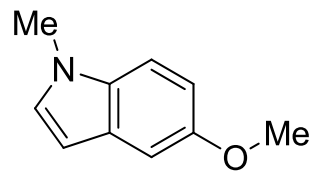

29

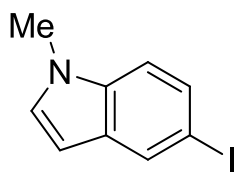

32

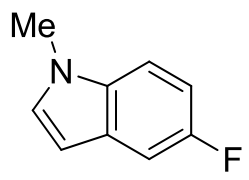

30

\section{1,2-Dimethyl-1H-indole (28)}<smiles>Cc1cc2ccccc2n1C</smiles>

28

Starting from commercially available 2-methylindole (656 mg, $5.00 \mathrm{mmol}), 1,2-$ dimethyl-1H-indole 28 (683 mg, $4.70 \mathrm{mmol}, 94 \%$ yield) was obtained as an offwhite solid. ${ }^{1} \mathbf{H}$ NMR (400 MHz $\left.\mathrm{CDCl}_{3}\right) \delta 7.69(\mathrm{~d}, J=7.8 \mathrm{~Hz}, 1 \mathrm{H}, \mathrm{Ar} H), 7.41$ $(\mathrm{dd}, J=8.1,1.0 \mathrm{~Hz}, \operatorname{Ar} H), 7.32(\mathrm{~m}, 1 \mathrm{H}, \operatorname{Ar} H), 7.24(\mathrm{ddd}, J=8.0,7.0,1.1 \mathrm{~Hz}, 1 \mathrm{H}$, $\mathrm{Ar} H), 6.42\left(\mathrm{~s}, 1 \mathrm{H}, \mathrm{NC}\left(\mathrm{CH}_{3}\right) \mathrm{CH}\right), 3.79(\mathrm{~s}, 3 \mathrm{H}, \mathrm{NCH}), 2.57$ (d, $\left.J=1.0 \mathrm{~Hz}, 3 \mathrm{H}, \mathrm{NCCH}_{3}\right) .{ }^{13} \mathbf{C} \mathbf{~ N M R}$ $\left(125 \mathrm{MHz}, \mathrm{CDCl}_{3}\right) \delta 138.1,136.9,128.1,120.6,119.8,119.4,108.8,99.7,29.5,12.9 . \mathbf{I R} v$ (neat) 3050 (w), 3020 (w), 2970 (m), 1610 (w), 1400 (s), 1340 (m), 1240 (m), 930 (m), 910 (w), $780(\mathrm{~m})$, $750(\mathrm{~m}), 730$ (s). ${ }^{1} \mathrm{H}$ NMR values are in accordance with the data reported in literature. ${ }^{4}$

\section{5-Methoxy-1-methyl-1H-indole (29)}

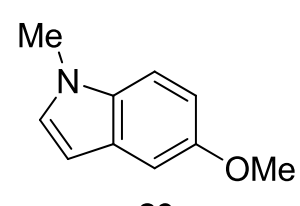

29

Starting from commercially available 5-methoxy- $1 H$-indole $(736 \mathrm{mg}, 5.00$ mmol), 5-methoxy-1-methyl-1H-indole 29 (730 mg, $4.53 \mathrm{mmol}, 91 \%$ yield) was obtained as a colorless crystalline solid. ${ }^{1} \mathbf{H}$ NMR $\left(400 \mathrm{MHz}, \mathrm{CDCl}_{3}\right) \delta \delta$ $7.30(\mathrm{~d}, 1 \mathrm{H}, J=8.5 \mathrm{~Hz}, \operatorname{Ar} H) 7.13$ (s, 1H, $\operatorname{ArH}), 7.05$ (s, $1 \mathrm{H}, \operatorname{Ar} H), 6.92$ (d, $1 \mathrm{H}, J=8.8 \mathrm{~Hz}, \operatorname{Ar} H), 6.43(\mathrm{~d}, 1 \mathrm{H}, J=1.0 \mathrm{~Hz}, \operatorname{Ar} H), 3.90$ (s, 3H, NMe), 3.80 (s, 3H, OMe). ${ }^{13} \mathrm{C}$ NMR $\left(101 \mathrm{MHz}, \mathrm{CDCl}_{3}\right) \delta 154.0,132.2,129.3,128.8,111.9,109.9,102.5,100.4,55.9,33.0$. IR v 2952 (w), 2918 (w), 2834 (w), 1622 (m), 1608 (w), 1577 (w), 1496 (s), 1459 (w), 1450 (m), 1449 (m), 1421 (s), 1347 (w), 1293 (w), 1243 (s), 1191 (m), 1152 (s), 1102 (w), 1026 (m), 942 (w), 855 (m), $845(\mathrm{w}), 805(\mathrm{~s})$. 


\section{5-Fluoro-1-methyl-1H-indole (30)}

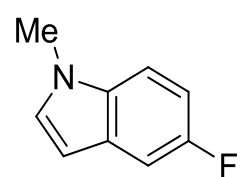

30

Starting from commercially available 5-fluoro- $1 H$-indole (676 mg, $5.00 \mathrm{mmol}), 5$ fluoro-1-methyl- $1 H$-indole 30 (683 mg, $4.58 \mathrm{mmol}$, 92\% yield) was obtained as a colorless solid. ${ }^{1} \mathbf{H}$ NMR (400 MHz, $\left.\mathrm{CDCl}_{3}\right) \delta 7.31(\mathrm{dd}, J=9.7,2.4 \mathrm{~Hz}, 1 \mathrm{H}, \mathrm{ArH})$, $7.26(\mathrm{~m}, 1 \mathrm{H}, \operatorname{Ar} H), 7.12(\mathrm{~d}, J=3.1 \mathrm{~Hz}, 1 \mathrm{H}, \operatorname{Ar} H), 7.01(\mathrm{dt}, J=9.1,2 \mathrm{~Hz}, 1 \mathrm{H}$, $\operatorname{Ar} H), 6.48(\mathrm{dd}, J=3.1,0.7 \mathrm{~Hz}, 1 \mathrm{H}, \operatorname{Ar} H) .3 .81\left(\mathrm{~s}, 3 \mathrm{H}, \mathrm{NCH}_{3}\right){ }^{13} \mathbf{C}$ NMR $\left(101 \mathrm{MHz}, \mathrm{CDCl}_{3}\right) \delta$ $158.0\left(\mathrm{~d}, J-\mathrm{F}_{6}=232 \mathrm{~Hz}\right), 133.4,130.4,128.7\left(\mathrm{~d}, J-\mathrm{F}_{6}=10 \mathrm{~Hz}\right), 109.9\left(\mathrm{~d}, J-\mathrm{F}_{6}=15 \mathrm{~Hz}\right), 109.8$, $105.5\left(\mathrm{~d}, J-\mathrm{F}_{6}=23 \mathrm{~Hz}\right), 100.8\left(\mathrm{~d}, J-\mathrm{F}_{6}=5.0 \mathrm{~Hz}\right), 33.1$. IR $3104(\mathrm{w}), 2946(\mathrm{w}), 2922(\mathrm{w}), 2907(\mathrm{w})$, 2887 (w), 2362 (w), 2343 (w), 1626 (w), 1576 (w), 1514 (m), 1492 (s), 1449 (m), 1423 (m), 1340 (m), 1283 (m), 1238 (s), 1228 (s), 1140 (m), 1129 (m), 1122 (m), 1100 (m), 1081 (m), 1013 (w), 949 (m), $859(\mathrm{~m}), 811(\mathrm{~s})$.

\section{5-Chloro-1-methyl-1H-indole (31)}

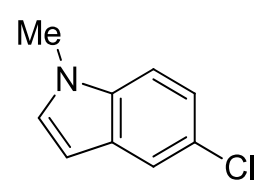

31

Starting from commercially available 5 -chloro- $1 H$-indole $(758 \mathrm{mg}, 5.00 \mathrm{mmol})$, 5-chloro-1-methyl-1H-indole 31 ( $800 \mathrm{mg}, 4.83 \mathrm{mmol}, 97 \%$ yield) was obtained as a colorless solid. ${ }^{1} \mathbf{H}$ NMR $\left(400 \mathrm{MHz}, \mathrm{CDCl}_{3}\right) \delta 7.64(\mathrm{~d}, J=2.1 \mathrm{~Hz}, 1 \mathrm{H}, \mathrm{Ar} H)$, $7.30-7.19(\mathrm{~m}, 2 \mathrm{H}, \operatorname{Ar} H), 7.10(\mathrm{~d}, J=3.1 \mathrm{~Hz}, 1 \mathrm{H}, \operatorname{Ar} H), 6.47(\mathrm{dd}, J=3.1,0.7$ $\mathrm{Hz}, 1 \mathrm{H}, \mathrm{ArH}), 3.80(\mathrm{~s}, 3 \mathrm{H}, \mathrm{NCH}) .{ }^{13} \mathbf{C}$ NMR $\left(101 \mathrm{MHz}, \mathrm{CDCl}_{3}\right) \delta 135.1,130.1,130.1,125.1$, 121.8, 120.2, 110.2, 100.6, 33.1. IR $v 3102$ (w), 2943 (w), 2913 (w), 2881 (w), 2817 (w), 1567 (w), 1513 (m), 1475 (s), 1441 (m), 1421 (s), 1379 (w), 1331 (m), 1278 (s), 1241 (s), 1199 (m), 1146 (m), 1106 (w), 1082 (m), 1063 (s), 1009 (m), 909 (m), 870 (m), 869 (m).

\section{5-Iodo-1H-indole (32)}

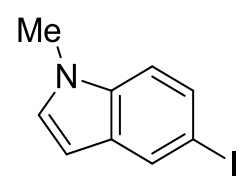

32

Starting from commercially available 5-iodo- $1 H$-indole $(257 \mathrm{mg}, 1.00 \mathrm{mmol})$, 5iodo-1-methyl- $1 \mathrm{H}$-indole 32 (380 $\mathrm{mg}, 0.755 \mathrm{mmol}, 76 \%$ yield) was obtained as a colorless solid. ${ }^{1} \mathbf{H}$ NMR $\left(400 \mathrm{MHz}, \mathrm{CDCl}_{3}\right) \delta 7.98(\mathrm{~s}, 1 \mathrm{H}, \mathrm{Ar} H), 7.49(\mathrm{~d}, J=8.6$ $\mathrm{Hz}, 1 \mathrm{H}, \operatorname{Ar} H), 7.13$ (d, $J=8.6 \mathrm{~Hz}, 1 \mathrm{H}, \operatorname{Ar} H), 7.04$ (s, 1H, $\operatorname{Ar} H), 6.43$ (s, 1H, ArH), $3.80\left(\mathrm{~s}, 3 \mathrm{H}, \mathrm{NCH}_{3}\right) .{ }^{13} \mathrm{C}$ NMR $\left(101 \mathrm{MHz}, \mathrm{CDCl}_{3}\right) \delta 135.8,131.0,129.8,129.7,129.6,111.3,100.3$, 
82.9, 33.0. IR $v 3093$ (w), 3053 (w), 2940 (w), 2919 (w), 2886 (w), 2876 (w), 2856 (w), 1557 (m), 1510 (s), 1473 (s), 1432 (m), 1420 (s), 1379 (w), 1329 (m), 1277 (s), 1242 (s), 1193 (w), 1151 (w), $1103(\mathrm{~m}), 1079(\mathrm{~m}), 1045$ (w), 1007 (m), 888 (s), $868(\mathrm{~m})$.

\section{$\underline{\text { N-Functionalized Indoles }}$}

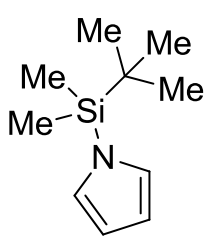

35

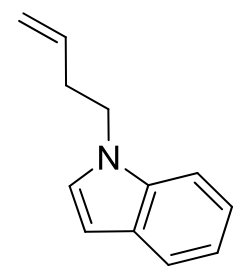

37

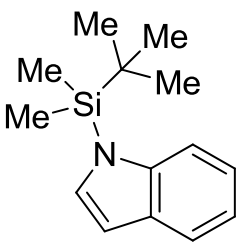

36

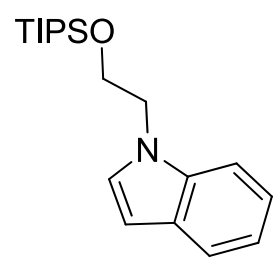

40

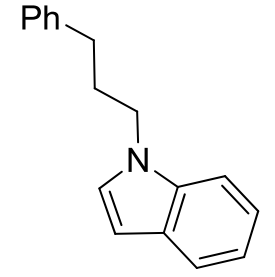

41

\section{1-(1-(tert-Butyldimethylsilyl)-1 H-pyrrole (35)}
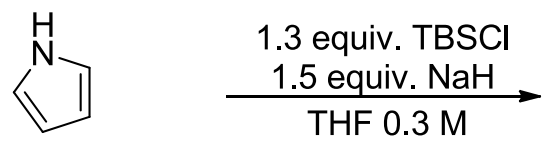

33

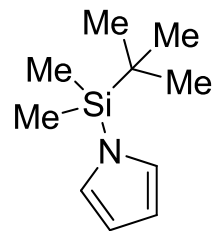

35

Following a reported procedure, ${ }^{5}$ commercially available $1 H$-pyrrole 33 (700 $\left.\mu 1,10.0 \mathrm{mmol}\right)$ was dissolved in dry THF (30 ml, $0.3 \mathrm{M})$. Sodium hydride $(60 \%$ suspension in mineral oil; $600 \mathrm{mg}$, $15.0 \mathrm{mmol}, 1.50$ equiv.) was slowly added under $\mathrm{N}_{2}$ flow at $0{ }^{\circ} \mathrm{C}$. After being stirred at $0{ }^{\circ} \mathrm{C}$ for 15

[5] A. F. G. Maier, S. Tussing, T. Schneider, U. Flörke, Z.-W. Qu, S. Grimme, J. Paradies, Angew. Chem. Int. Ed. 2016, 55, 12219-12223. 
min, the reaction mixture was allowed to warm to r.t for $1.50 \mathrm{~h}$. Then it was cooled back to $0^{\circ} \mathrm{C}$ and tert-butylchlorodimethylsilane (1.96 g, $13.0 \mathrm{mmol}, 1.30$ equiv.) was added. The mixture was warmed to r.t. and stirred overnight. The reaction was quenched by addition of water $(10 \mathrm{~mL})$ at $0^{\circ} \mathrm{C}$. The aqueous layer was extracted with $\mathrm{Et}_{2} \mathrm{O}(3 \times 10 \mathrm{~mL})$, the combined organic layers were dried over $\mathrm{MgSO}_{4}$, and the solvent was removed under reduced pressure. The crude product was purified via flash column chromatography (Pentane:EtOAc 8:1), to give the desired 1-(tertbutyldimethylsilyl)-1H-pyrrole 35 (1.30 g, $7.17 \mathrm{mmol}, 72 \%$ yield). ${ }^{1} \mathbf{H}$ NMR (400 MHz, $\left.\mathrm{CDCl}_{3}\right) \delta$ $6.86-6.62(\mathrm{~m}, 2 \mathrm{H}, \operatorname{ArH}), 6.39-6.21(\mathrm{~m}, 2 \mathrm{H}, \operatorname{Ar} H), 0.86\left(\mathrm{~s}, 9 \mathrm{H}, \operatorname{SiC}\left(\mathrm{CH}_{3}\right)_{3}\right), 0.41(\mathrm{~s}, 6 \mathrm{H}$, $\left.\mathrm{Si}\left(\mathrm{CH}_{3}\right)_{2}\right)$. IR $v 3100(\mathrm{w}), 2956$ (m), 2931 (m), 2858 (m), 1707 (w), 1473 (m), 1364 (w), 1259 (s), 1222 (w), 1190 (s), 1084 (s), 1048 (s), 1008 (w), 942 (w), 839 (s). ${ }^{1} \mathrm{H}$ NMR values are in accordance with the data reported in literature. ${ }^{6}$

\section{1-(tert-Butyldimethylsilyl)-1H-Indole (36)}

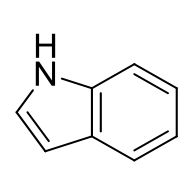

34

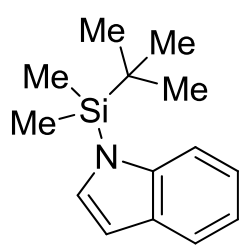

36

Following a reported procedure, ${ }^{7}$ commercially available $1 H$-indole $34(586 \mathrm{mg}, 5.00 \mathrm{mmol})$ was dissolved in dry THF (10 mL, $0.5 \mathrm{M})$. Sodium hydride (60\% suspension in mineral oil; $300 \mathrm{mg}$, $7.50 \mathrm{mmol}, 1.50$ equiv.) was slowly added under $\mathrm{N}_{2}$ atmosphere at $0{ }^{\circ} \mathrm{C}$. After being stirred at 0 ${ }^{\circ} \mathrm{C}$ for $15 \mathrm{~min}$, the reaction mixture was allowed to warm to r.t for $1.5 \mathrm{~h}$. Then it was cooled back to $0{ }^{\circ} \mathrm{C}$ and tert-butylchlorodimethylsilane ( $980 \mathrm{mg}, 6.50 \mathrm{mmol}, 1.30$ equiv.) was added. The mixture was warmed to r.t. and stirred overnight. After cooling again to $0{ }^{\circ} \mathrm{C}$, the reaction was quenched with water $(10 \mathrm{~mL})$, extracted with $\mathrm{Et}_{2} \mathrm{O}(3 \times 10 \mathrm{~mL})$, the combined organic layers were dried over $\mathrm{MgSO}_{4}$, and the solvent was removed under reduced pressure. The residue was purified via flash column chromatography (Pentane:EtOAc 4:1), to give the desired 1-(tertbutyldimethylsilyl)-1 $H$-indole 36 (869 mg, 3.76 mmol, 75\% yield). ${ }^{1} \mathbf{H}$ NMR (400 MHz, $\mathrm{CDCl}_{3}$ )

[6] G. Simchen, M. W. Majchrzak, Tetrahedron Lett. 1985, 26, 5035-5036.

[7] S. Islam, I. Larrosa, Chem. - Eur. J. 2013, 19, 15093-15096 
$\delta 7.79(\mathrm{~m}, 1 \mathrm{H}, \operatorname{Ar} H), 7.72(\mathrm{dt}, \mathrm{J}=8.2,1.1 \mathrm{~Hz}, 1 \mathrm{H}, \operatorname{Ar} H), 7.37$ (d, J=3.2 Hz, 1H, ArH), 7.32 (ddd, $J=8.3,7.0,1.6 \mathrm{~Hz}, 1 \mathrm{H}, \operatorname{Ar} H), 7.25(\mathrm{~m}, 1 \mathrm{H}), 6.78(\mathrm{dd}, J=3.3,1.0 \mathrm{~Hz}, 1 \mathrm{H}, \operatorname{Ar} H), 1.11$ (s, 9H, $\left.\mathrm{SiCCH}_{3}\right), 0.78\left(\mathrm{~s}, 6 \mathrm{H}, \mathrm{Si}\left(\mathrm{CH}_{3}\right)_{2}\right)$. IR $3064.6(\mathrm{w}), 2950.5(\mathrm{w}), 2929.7$ (m), $2855.9(\mathrm{w}), 1512.0$ (s), 1427.6 (s), 1449.9 (m), 1284.3 (s), 1271.6 (s), 1255.8 (m), 1158.9 (s), 1140.9 (s), 984.2 (w), 840.1 (m). ${ }^{1} \mathrm{H}$ NMR values are in accordance with the data reported in literature. ${ }^{8}$

\section{1-(But-3-en-1-yl)-1H-Indole (37)}

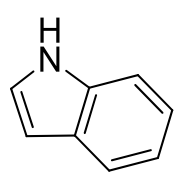

34

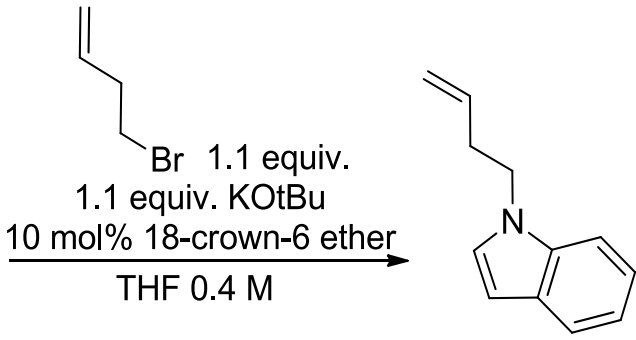

37

Following a reported procedure, ${ }^{9}$ potassium tertbutoxide $(1.24 \mathrm{mg}, 11.0 \mathrm{mmol}, 1.10$ equiv.) was added to a solution of 18-crown-6 ether $(26.4 \mathrm{mg}, 0.100 \mathrm{mmol}, 10 \mathrm{~mol} \%$.) in dry THF ( $25 \mathrm{~mL}, 0.4$ M) under a nitrogen atmosphere. Then, commercially available $1 H$-indole 34 (1.17 g, $10.0 \mathrm{mmol}$, 1.00 equiv.) was added under vigorous stirring. The reaction was cooled to $0^{\circ} \mathrm{C}$ in an ice bath. A solution of 4-bromobut-1-ene (1.20 mL, $11.0 \mathrm{mmol}, 1.10$ equiv.) in THF (5 mL) was added dropwise to the reaction mixture and the latter was stirred overnight. After cooling again to $0{ }^{\circ} \mathrm{C}$, the reaction was quenched with water $(20 \mathrm{~mL})$, extracted with $\mathrm{Et}_{2} \mathrm{O}(3 \times 20 \mathrm{~mL})$, the combined organic layers were dried over $\mathrm{MgSO}_{4}$, and the solvent was removed under reduced pressure. The residue was purified via flash column chromatography (Pentane:EtOAc 9:1) to give the desired 1(but-3-en-1-yl)-1 $H$-indole 37 (280 mg, $1.64 \mathrm{mmol}, 17 \%$ yield) as a yellow oil. ${ }^{1} \mathbf{H}$ NMR $(400 \mathrm{MHz}$, $\left.\mathrm{CDCl}_{3}\right) \delta 7.84(\mathrm{~d}, J=8.0 \mathrm{~Hz}, 1 \mathrm{H}, \operatorname{Ar} H), 7.52(\mathrm{~d}, J=8.2 \mathrm{~Hz}, 1 \mathrm{H}, \operatorname{Ar} H), 7.41(\mathrm{t}, J=7.6 \mathrm{~Hz}, 1 \mathrm{H}$, $\operatorname{Ar} H), 7.31$ (t, $J=7.4 \mathrm{~Hz}, 1 \mathrm{H}, \operatorname{Ar} H), 7.23(\mathrm{~d}, J=3.2 \mathrm{~Hz}, 1 \mathrm{H}, \operatorname{Ar} H), 6.68(\mathrm{~d}, J=3.2 \mathrm{~Hz}, 1 \mathrm{H}, \operatorname{Ar} H)$, 5.94 (ddt, $J=17.1,10.2,6.8 \mathrm{~Hz}, 1 \mathrm{H}, \mathrm{ArH}), 5.32-5.12\left(\mathrm{~m}, 2 \mathrm{H}, \mathrm{NCH}_{2} \mathrm{CH}_{2} \mathrm{CH}=\mathrm{CH}_{2}\right), 4.31-4.27$ (m, 2H, $\mathrm{CH}_{2}$ ), 2.72 (q, $J=7.1 \mathrm{~Hz}, 2 \mathrm{H}, \mathrm{CH}_{2}$ ). IR $v 2936$ (w), 1640 (s), 1612 (s), 1508 (m), 1458

[8] D. Saha, R. Ghosh, A. Sarkar, Tetrahedron 2013, 69, 3951-3960.

[9] W. C. Guida, D. J. Mathre, J. Org. Chem. 1980, 45, 3172-3176. 
(s), $910(\mathrm{~m}), 740(\mathrm{~m}), 712(\mathrm{~m}) .{ }^{1} \mathrm{H}$ NMR values are in accordance with the data reported in literature. ${ }^{10}$

(2-Iodoethoxy)triisopropylsilane (38)

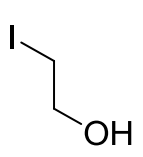

38

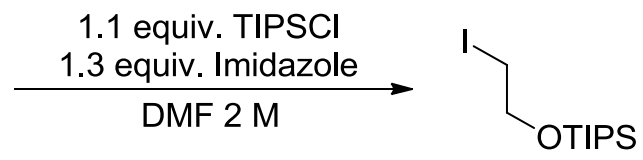

39

Following a reported procedure, ${ }^{11}$ commercially available 2 -iodoethanol $\mathbf{3 8}(1.10 \mathrm{~mL}, 10.0 \mathrm{mmol}$, 1.00 equiv.) was added to a solution of imidazole ( $885 \mathrm{mg}, 13.0 \mathrm{mmol}, 1.30$ equiv.) in DMF (5 $\mathrm{mL}, 2.0 \mathrm{M})$ under nitrogen atmosphere. chloro-triisopropylsilane (2.80 mL, $13.0 \mathrm{mmol}, 1.30$ equiv.) was then added dropwise. After $1 \mathrm{~h}$, the reaction turned into a thick suspension and a colorless solid precipitated. The mixture was allowed to warm to room temperature removed and was stirred for an additional hour. Water $(5 \mathrm{~mL})$ was added to dissolve the solid. The organic layer was separated and eluted through a $\mathrm{SiO}_{2}$ plug with pentane $(100 \mathrm{~mL})$. The solvent was removed under reduced pressure to give (2-iodoethoxy)triisopropylsilane 39 (3.15 g, $9.60 \mathrm{mmol}, 96 \%$ yield) as a slightly yellow oil. ${ }^{1} \mathbf{H}$ NMR (400 MHz, $\left.\mathrm{CDCl}_{3}\right) \delta 3.85(\mathrm{t}, J=6.9 \mathrm{~Hz}, 2 \mathrm{H}, \mathrm{OCH}), 3.15(\mathrm{t}, J=7.0$ $\mathrm{Hz}, 2 \mathrm{H}, \mathrm{ICH}), 1.11-0.88(\mathrm{~m}, 21 \mathrm{H}, \mathrm{TIPS}) .{ }^{13} \mathbf{C}$ NMR $\left(101 \mathrm{MHz}, \mathrm{CDCl}_{3}\right) \delta 64.6,18.0,12.1,6.9 . \mathbf{I R}$ v 2958 (m), 2942 (m), 2891 (w), 2866 (m), 1464 (m), 1384 (w), 1275 (w), 1249 (w), 1190 (w), 1169 (w), 1123 (s), 1092 (s), 1069 (s), 1013 (w), 999 (m), 943 (w), 920 (w), 882 (s), 857 (w).

1-(2-((Triisopropylsilyl)oxy)ethyl)- $1 H$-Indole (40)

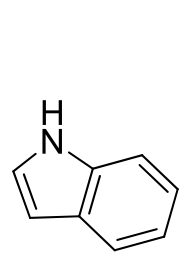

34

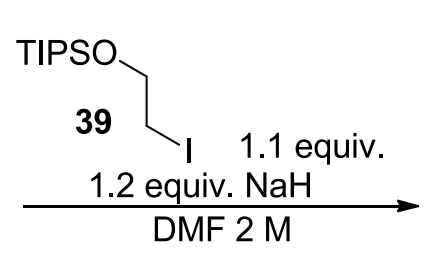

DMF 2 M

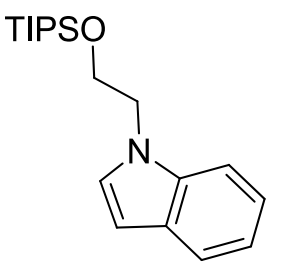

40

[10] H. A. Kerchner, J. Montgomery, Org. Lett. 2016, 18, 5760-5763.

[11] J. W. Bode, E. M. Carreira, J. Org. Chem. 2001, 66, 6410-6424. 
Commercially available $1 H$-indole $34(586 \mathrm{mg}, 5.00 \mathrm{mmol})$ was dissolved in $\mathrm{N}, \mathrm{N}-$ dimethylformamide (5 mL, $0.5 \mathrm{M}$ in total). Sodium hydride ( $60 \%$ suspension in mineral oil; 144 $\mathrm{mg}, 6.00 \mathrm{mmol}, 1.20$ equiv.) was added at r.t. and the reaction mixture was stirred for one hour. N,N-Dimethylformamide $(5 \mathrm{~mL})$ was then added to dissolve the resulting colorless precipitate. The reaction was cooled to $0{ }^{\circ} \mathrm{C}$ and (2-iodoethoxy)triisopropylsilane 39 (1.90 g, $5.50 \mathrm{mmol}, 1.10$ equiv.) was added dropwise. The reaction mixture was stirred overnight, allowing it to warm to r.t. The reaction was then quenched with water $(20 \mathrm{~mL})$ and the reaction mixture was extracted with EtOAc $(3 \times 25 \mathrm{~mL})$. The combined organic layers were washed with water $(10 \mathrm{~mL})$, brine $(3 \times 10$ $\mathrm{mL}$ ), and dried over $\mathrm{MgSO}_{4}$. The solvent was then removed under reduced pressure. Flash column chromatography (Pentane:EtOAc 9:1) afforded 1-(2-((triisopropylsilyl)oxy)ethyl)- $1 H$-indole 40 $\left(1.20 \mathrm{~g}, 3.78 \mathrm{mmol}, 76 \%\right.$ yield) as a colorless oil. ${ }^{1} \mathbf{H}$ NMR $\left(400 \mathrm{MHz}, \mathrm{CDCl}_{3}\right) \delta 7.66(\mathrm{~m}, 1 \mathrm{H}$, $\operatorname{Ar} H$ ), 7.38 (dd, $J=8.2,0.8 \mathrm{~Hz}, 1 \mathrm{H}, \operatorname{Ar} H), 7.25-7.19$ (m, 2H, ArH), 7.13 (m, 1H, ArH), 6.52 (dd, $J=3.1,0.8 \mathrm{~Hz}, 1 \mathrm{H}, \mathrm{ArH}), 4.30$ (t, $\left.J=6.0 \mathrm{~Hz}, 2 \mathrm{H}, \mathrm{CH}_{2}\right), 4.04$ (t, $\left.J=5.8 \mathrm{~Hz}, 2 \mathrm{H}, \mathrm{CH}_{2}\right), 1.17-0.85$ (m, 21H, TIPS). ${ }^{13} \mathbf{C}$ NMR $\left(101 \mathrm{MHz}, \mathrm{CDCl}_{3}\right) \delta 136.1,128.7,128.6,121.3,120.9,119.2,109.3$, 101.0, 62.8, 48.8, 17.9, 11.9. IR v 3056 (w), 2891 (m), 2865 (s), $1514(\mathrm{w}), 1464$ (s), 1439 (w), 1387 (w), 1360 (w), 1334 (w), 1317 (m), 1115 (s), 1077 (m), 1013 (m), 923 (m), 819 (w).

\section{1-(3-Phenylpropyl)-1H-indole (41)}

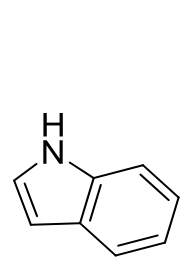

34

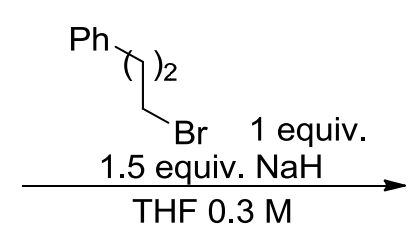

THF $0.3 \mathrm{M}$

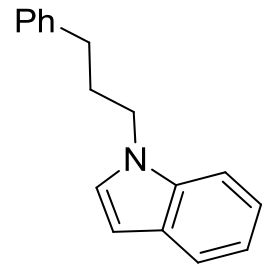

41

Following a reported procedure (12), commercially available $1 H$-indole 34 (585 mg, $5.00 \mathrm{mmol}$, 1.00 equiv.) was dissolved in THF (15 mL). Sodium hydride (60\% suspension in mineral oil; 300 $\mathrm{mg}, 7.50 \mathrm{mmol}, 1.50$ equiv.) was added at $0{ }^{\circ} \mathrm{C}$ and the reaction mixture was stirred for $30 \mathrm{~min}$. (3bromopropyl)benzene ( $760 \mu \mathrm{L}, 5.00 \mathrm{mmol}, 1.00$ equiv.) was then added dropwise. After $15 \mathrm{~min}$ the ice bath was removed and the reaction mixture was stirred for 4 hours at r.t.. The reaction was cooled back to $0{ }^{\circ} \mathrm{C}$, quenched with water, diluted with EtOAc $(10 \mathrm{~mL})$, extracted with water $(2 \mathrm{x}$ $10 \mathrm{~mL})$, washed with brine $(10 \mathrm{~mL})$, and dried over $\mathrm{MgSO}_{4}$. After filtration, the solvent was

[12] Y. R. Jorapur, J. M. Jeong, D. Y. Chi, Tetrahedron Lett. 2006, 47, 2435-2438. 
removed under reduced pressure. Flash column chromatography (Pentane:EtOAc 20:1) afforded 1-(3-phenylpropyl)-1H-indole 41 (1.12 g, $4.76 \mathrm{mmol}, 95 \%$ yield) as a colorless oil. ${ }^{1} \mathbf{H}$ NMR (400 $\left.\mathrm{MHz}, \mathrm{CDCl}_{3}\right) \delta 7.87$ (d, $\left.J=8 \mathrm{~Hz}, 1 \mathrm{H}, \mathrm{Ar} H\right), 7.53-7.23$ (m, 9H, $\left.\mathrm{ArH}\right), 6.72$ (dd, $J=3.1,0.8 \mathrm{~Hz}$, $1 \mathrm{H}, \mathrm{Ar} H), 4.26\left(\mathrm{t}, J=7.1 \mathrm{~Hz}, 2 \mathrm{H}, \mathrm{NCH}_{2}\right), 2.77\left(\mathrm{t}, J=8 \mathrm{~Hz}, 2 \mathrm{H}, \mathrm{CH}_{2} \mathrm{Ph}\right), 2.34$ (qi, $J=7.8 \mathrm{~Hz}, 2 \mathrm{H}$, $\left.\mathrm{CH}_{2} \mathrm{CH}_{2} \mathrm{CH}_{2}\right) .{ }^{13} \mathbf{C}$ NMR $\left(101 \mathrm{MHz}, \mathrm{CDCl}_{3}\right) \delta$ 141.2, 136.2, 128.7, 128.7, 128.6, 128.0, 126.3, 121.6, 121.2, 119.5, 109.6, 101.3, 45.8, 33.2, 31.7 (one aromatic Carbon signal not resolved). IR 3085 (w), 3057 (w), 3026 (w), 3004 (w), 2946 (w), 2945 (w), 2870 (w), 1780 (w), 1738 (s), 1717 (s), $1612(\mathrm{w}), 1603$ (w), 1511 (m), 1497 (m), 1483 (m), 1464 (s), 1455 (s), 1400 (m), 1377 (s), 1354 (s), 1336 (s), 1315 (s), 1254 (s), 1207 (s), 1179 (m), 1166 (m), 1143 (m), 1143 (m), 1122 (m), $1114(\mathrm{~m}), 1080$ (m), 1031 (m), 1020 (m), 1004 (w), 952 (w), 928 (w), 909 (w), $885(\mathrm{~m}), 855(\mathrm{w})$, $838(\mathrm{w}), 821(\mathrm{w}), 811(\mathrm{w}), 802(\mathrm{w})$. 


\subsection{Optimization in the synthesis of IndoleBX 9a.}

Table S1: Screening of additives

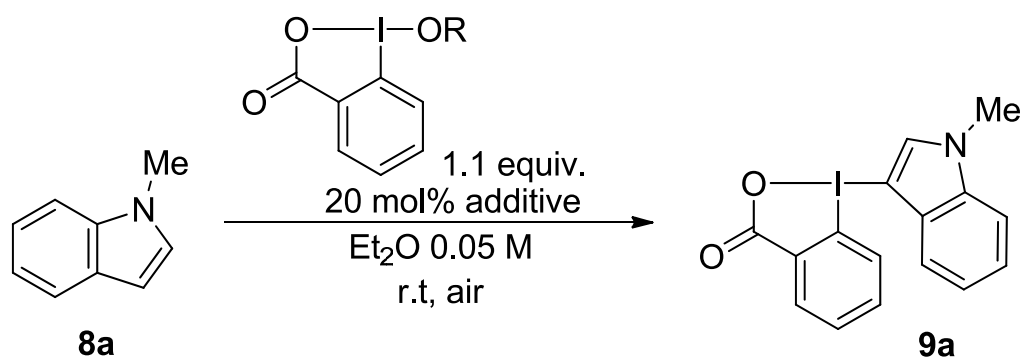

\begin{tabular}{|c|c|c|c|}
\hline Entry & Reagent & Additive & Yield\% $^{\mathrm{a}}$ \\
\hline 1 & $\operatorname{HOBX}(7)$ & TMSOTf & $-b$ \\
\hline 2 & HOBX (7) & $\mathrm{Zn}(\mathrm{OTf})_{2}$ & $-b$ \\
\hline 3 & AcOBX (5) & - & $-b$ \\
\hline 4 & AcOBX (5) & TMSOTf & $16 \%$ \\
\hline 5 & AcOBX (5) & $\mathrm{Zn}(\mathrm{OTf})_{2}$ & $36 \% \mathrm{o}^{\mathrm{b}}$ \\
\hline 6 & AcOBX (5) & $\mathrm{Cu}(\mathrm{OTf})_{2}$ & $36 \%{ }^{c}$ \\
\hline 7 & AcOBX (5) & $\mathrm{AgNTf}_{2}$ & $-\mathrm{c}$ \\
\hline 8 & AcOBX (5) & $\mathrm{AgF}$ & $-^{c}$ \\
\hline 9 & AcOBX (5) & $\mathrm{CsF}$ & $-c$ \\
\hline 10 & AcOBX (5) & TBAF & $-\mathrm{c}$ \\
\hline
\end{tabular}

a) Substrate 8a $(0.100 \mathrm{mmol})$, reagents $7-5(0.110 \mathrm{mmol})$, additive $(20 \mathrm{~mol} \%)$, and $\mathrm{Et}_{2} \mathrm{O}(0.05 \mathrm{M})$ at $25{ }^{\circ} \mathrm{C}$. Isolated yield after flash chromatography is given. b) No conversion: starting materials recovered. c) Complete decomposition of the hypervalent iodine reagents.

Table S2: Screening of solvents and additive loading

\begin{tabular}{cccc}
\hline Entry & Solvent & $\mathbf{X ~ m o l \% ~ Z n ( O T f ) ~}$ & Yield\% $^{\mathbf{a}}$ \\
\hline 1 & THF & 10 & - b $^{-}$ \\
2 & THF & 20 & $-\mathrm{b}$ \\
3 & THF & 1 equiv. & $-{ }^{\mathrm{b}}$ \\
4 & DCM & 10 & Full conversion $^{\mathrm{c}}$
\end{tabular}


6 DCM 1 equiv. decomposition

a) Substrate 8a $(0.100 \mathrm{mmol})$, AcOBX $5(0.110 \mathrm{mmol}), \mathrm{Zn}(\mathrm{OTf})_{2}(\mathbf{x} \mathrm{mol} \%)$, and solvent $(0.05 \mathrm{M})$ at $25{ }^{\circ} \mathrm{C}$. Isolated yield after flash chromatography is given. b) No conversion: starting materials recovered. c) 2 equiv. of OAcBX $\mathrm{xx}$ are needed. 


\subsection{Preparation of PyrroleBX and IndoleBX Reagents.}

\section{General Procedure GP2 for the Synthesis of Heterocyclic-BX Reagents}

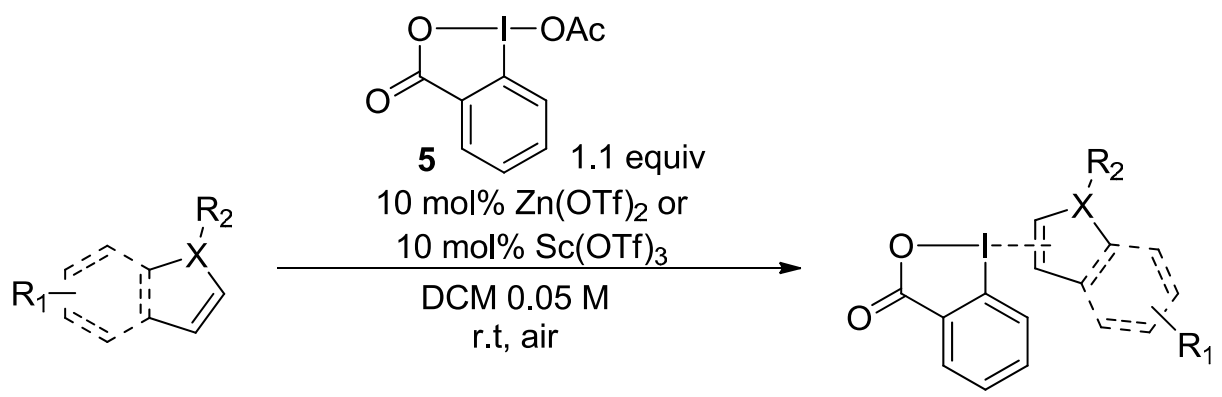

Note: prior to the reaction, the glassware requires to be carefully cleaned with aqua regia to remove all metal traces; the commercially available heterocyclic starting material were purified through a short plug of silica prior to being used. Compound 5 can be prepared or purchased from commercial suppliers. Slow decomposition of $\mathbf{5}$ is observed at room temperature; for this reason, compound 5 should be stored at 3-5 ${ }^{\circ} \mathrm{C}$ and best results are obtained if it is prepared freshly.

In an open air flask, the corresponding heterocycle (1.00 mmol, 1.00 equiv.), 1-acetoxy-1,2benziodoxol-3-(1H)-one $5 \quad(337 \quad \mathrm{mg}, \quad 1.10 \quad \mathrm{mmol}, \quad 1.10 \quad$ equiv.) and zinc(II) trifluoromethanesulfonate $(72.7 \mathrm{mg}, 0.200 \mathrm{mmol}, 20 \mathrm{~mol} \%$.) were dissolved in DCM (20 mL 0.05 M). The reaction was stirred while being monitored by TLC (Pentane:EtOAc 9:1 for the starting materials, DCM:MeOH 9:1 for the products). Upon consumption of the starting material, the crude was directly submitted to short-path flash chromatography (DCM:MeOH 10:1 or EtOAc:MeOH 10:1 for the separation of pyrrole-based reagents) to afford the desired Heterocyclic-BX compounds 9a-9r.The structure of compounds $9 b$ and $9 n$ was confirmed by $X$-Ray analysis (see par. 5 of the Supplementary Informations). 
<smiles>Cn1cc(I2OC(=O)c3ccccc32)c2ccccc21</smiles>

9a

$87 \%$ yield<smiles>O=C1OI(c2cn(CCCc3ccccc3)c3ccccc23)c2ccccc21</smiles>

$9 e$

$88 \%$ yield<smiles>Cn1cc(I2COC(=O)c3ccccc32)c2cc(Cl)ccc21</smiles>

$9 \mathrm{i}$

$62 \%$ yield<smiles>O=C1OI(c2c[nH]c3ccccc23)c2ccccc21</smiles>

9b

$78 \%$ yield<smiles>Cc1c(I2COC(=O)c3ccccc32)c2ccccc2n1C</smiles>

$9 f$

91\% yield<smiles>Cn1cc(I2COC(=O)c3ccccc32)c2cc(I)ccc21</smiles>

9j

$76 \%$ yield<smiles>C=CCCn1cc(I2COC(=O)c3ccccc32)c2ccccc21</smiles>

9c

$86 \%$ yield<smiles>COc1ccc2c(c1)c(I1COC(=O)c3ccccc31)cn2C</smiles>

9g

$87 \%$ yield<smiles>Cn1cc(I2COC(=O)c3ccccc32)c2cc([SbH3])ccc21</smiles>

9k

$54 \%$ yield

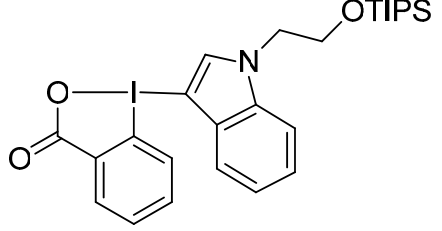

9d

$60 \%$ yield<smiles>Cn1cc(I2COC(=O)c3ccccc32)c2cc(F)ccc21</smiles>

9h

$85 \%$ yield<smiles>Cn1cc(I2COC(=O)c3ccccc32)c2cccnc21</smiles>

91

$26 \%$ yield

\section{OTHERS}<smiles>Cn1c2ccccc2c2cc(I3COC(=O)c4ccccc43)ccc21</smiles>

9m

$33 \%$ yield<smiles></smiles>

9q<smiles>O=C1OI(c2cc[nH]c2)c2ccccc21</smiles>

9n<smiles>O=C1Oc2c(I)cccc2I1Cn1cccc1Cc1ccccc1</smiles>

9r<smiles>Cn1ccc(I2COC(=O)c3ccccc32)c1</smiles>

9o

$95 \%$ yield 1:1 ratio $\mathrm{C} 2: \mathrm{C} 3$<smiles>O=C1OI(c2cccs2)c2ccccc21</smiles>

9s<smiles>Cn1cccc1I1COC(=O)c2ccccc21</smiles>

$9 p$<smiles>Cc1cc(I2COC(=O)c3ccccc32)c(C)o1</smiles>

$9 t$ $14 \%$ yield

$85 \%$ yield 1:3 ratio $\mathrm{C} 2: \mathrm{C} 3$

Figure S1: Scope of Heterocyclic-BX reagents 


\section{1-(3-1-Methyl-1 $H$-indole)-1 $H$-1 $\lambda_{3}$-benzo $[b]$ iodo-3(2H)-one (9a)}<smiles>Cn1cc(I2COC(=O)c3ccccc32)c2ccccc21</smiles>

9a

The synthesis of 1-(3-1-methyl-1H-indole)-1H-1 $\lambda_{3}$-benzo[b]iodo-3(2H)one 9 a was scaled up to $10 \mathrm{mmol}$ without reoptimization of the protocol.

Starting from commercially available 1-methyl-1-H-indole 8a (1.35 g, 10.0 mmol), after 16 hours 1-(3-1-methyl- $1 H$-indole)- $1 H$ - $1 \lambda_{3}$-benzo[b]iodo$3(2 H)$-one 9a (3.28 g, $8.70 \mathrm{mmol}, 87 \%$ yield) was obtained as a brown foam. Rf: 0.4 (DCM:MeOH 9:1). ${ }^{1} \mathbf{H}$ NMR $\left(400 \mathrm{MHz}, \mathrm{CDCl}_{3}\right) \delta 8.40(\mathrm{dd}, J=7.5,1.7 \mathrm{~Hz}, 1 \mathrm{H}$, $\operatorname{Ar} H), 7.82$ (s, 1H, NCHCI), 7.55 - 7.48 (m, 2H, ArH), 7.39 - 7.35 (m, 2H, ArH), $7.34-7.23$ (m, $\left.2 \mathrm{H}, \mathrm{ArH}+\mathrm{CDCl}_{3}\right), 6.84(\mathrm{~d}, J=8.3 \mathrm{~Hz}, 1 \mathrm{H}, \mathrm{ArH}), 4.02(\mathrm{~s}, 3 \mathrm{H}, \mathrm{NCH}) .{ }^{13} \mathbf{C}$ NMR $(101 \mathrm{MHz}$, $\mathrm{CDCl} 3) \delta 166.8,138.6,137.6,133.4,133.3,132.5,130.5,129.3,125.2,124.3,122.6,119.9,116.1$, 110.7, 78.9, 33.9. IR v 3107 (w), 3059 (w), 2948 (w), 1599 (s), 1552 (m), 1506 (m), 1454 (w), 1392 (m), 1277 (s), 1245 (s), 1225 (s), 1166 (s), 1131 (m), 1031 (s), 1004 (w), 842 (w). HRMS (ESI) calcd for $\mathrm{C}_{16} \mathrm{H}_{13} \mathrm{NNO}_{2}^{+}[\mathrm{M}+\mathrm{H}]^{+} 377.9986$; found 377.9990. The structure of the obtained regioisomer was assigned by NMR correlation to compound $\mathbf{9 b}$. DSC-analysis was performed on compound 9a: see par.5 of Supplementary informations.

\section{1-(3-1H-indole)-1H-1 $\lambda_{3}$-benzo[b]iodo-3(2H)-one (9b)}<smiles>O=C1OI(c2c[nH]c3ccccc23)c2ccccc21</smiles>

$9 b$

Starting from 1-(tert-butyldimethylsilyl)-1H-indole $36(231 \mathrm{mg}, 1.00 \mathrm{mmol})$ and using $\mathrm{Sc}(\mathrm{OTf})_{3}$ as the Lewis acid (20 mol \%), after 16 hours 1-(3-1Hindole)- $1 H-1 \lambda_{3}$-benzo[b]iodo-3(2H)-one $9 \mathbf{b}$ (294 mg, $8.10 \mathrm{mmol}, 78 \%$ yield) was obtained as a brown solid. Rf: 0.4 (DCM:MeOH 9:1). Mp: $174.2^{\circ} \mathrm{C}$ (decomposition) ${ }^{1} \mathbf{H}$ NMR (400 MHz, DMSO- $\left.d_{6}\right) \delta 12.33(\mathrm{~s}, 1 \mathrm{H}$, $\mathrm{NH}), 8.21(\mathrm{~s}, 1 \mathrm{H}, \mathrm{NCHCI}), 8.08(\mathrm{dd}, J=7.3,1.7 \mathrm{~Hz}, 1 \mathrm{H}, \mathrm{Ar} H), 7.59(\mathrm{~d}, J=8.2 \mathrm{~Hz}, 1 \mathrm{H}, \operatorname{Ar} H), 7.52$ (t, $J=7.3 \mathrm{~Hz}, 1 \mathrm{H}, \operatorname{Ar} H), 7.43$ (d, $J=7.5 \mathrm{~Hz}, 1 \mathrm{H}, \operatorname{Ar} H$ ), 7.36 (ddd, $J=8.5,7.3,1.7 \mathrm{~Hz}, 1 \mathrm{H}, \mathrm{Ar} H$ ), 7.26 (ddd, $J=8.2,7.0,1.2 \mathrm{~Hz}, 1 \mathrm{H}, \operatorname{Ar} H), 7.15$ (t, $J=7.5 \mathrm{~Hz}, 1 \mathrm{H}, \operatorname{Ar} H), 6.72$ (d, $J=8.5 \mathrm{~Hz}, 1 \mathrm{H}$, $\operatorname{Ar} H) .{ }^{13} \mathbf{C}$ NMR $\left(101 \mathrm{MHz}, \mathrm{DMSO}-d_{6}\right) \delta 166.1,136.6,134.5,133.3,131.4,130.2,128.5,126.3$, 123.4, 122.3, 121.6, 119.2, 116.1, 112.9, 80.0. IR $v 3302$ (w), 2975 (w), 2903 (w), 1722 (w), 1609 (m), 1584 (m), 1557 (w), 1490 (w), 1457 (w), 1385 (w), 1277 (s), 1258 (s), 1230 (m), 1174 (m), 1087 (w), 1036 (s), 880 (w), 841 (w). HRMS (ESI) calcd for $\mathrm{C}_{15} \mathrm{H}_{11} \mathrm{INO}_{2}{ }^{+}[\mathrm{M}+\mathrm{H}]^{+} 363.9829$; 
found 363.9832. The structure of the reagent was determined through X-Ray diffraction of a single crystal (CCDC number: 1540821).

\section{1-(3-1-(But-3-en-1-yl)-1H-indole)-1H-1 $\lambda_{3}$-benzo[b]iodo-3(2H)-one (9c)}

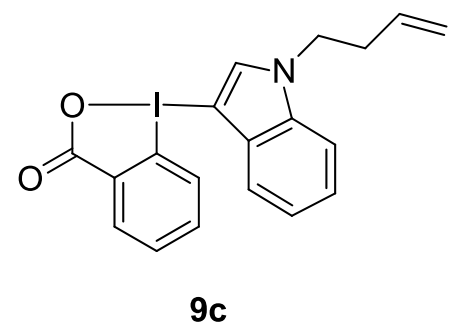

Starting from 1-(but-3-en-1-yl)-1H-indole 37 (171 mg, $1.00 \mathrm{mmol}$ ), after 16 hours 1-(3-1-(but-3-en-1-yl)-1H-indole)-1H-1 $\lambda_{3} \quad$ benzo[b]iodo-3(2H)-one 9c (359 mg, $0.860 \mathrm{mmol}, 86 \%$ yield) was obtained, as a yellow amorphous solid. Rf: 0.44 (DCM:MeOH 9:1). ${ }^{1} \mathbf{H}$ NMR $\left(400 \mathrm{MHz}, \mathrm{CDCl}_{3}\right) \delta 8.35(\mathrm{dd}, J=7.4,1.7 \mathrm{~Hz}, 1 \mathrm{H}, \mathrm{Ar} H)$, 7.91 (s, 1H, NCHCI), 7.54 (m, 1H, ArH), 7.49 (td, $J=7.3,0.9 \mathrm{~Hz}, 1 \mathrm{H}, \operatorname{Ar} H), 7.46-7.39$ (m, 2H, $\operatorname{Ar} H$ ), $7.32-7.19$ (m, 2H, ArH), 6.73 (dd, $J=8.3,0.9$ Hz, 1H, ArH), 5.82 (ddt, $J=17.1,10.3,6.9$ $\left.\mathrm{Hz}, 1 \mathrm{H}, \mathrm{NCH}_{2} \mathrm{CH}_{2} \mathrm{CH}=\mathrm{CH}_{2}\right), 5.12-4.97\left(\mathrm{~m}, 2 \mathrm{H}, \mathrm{NCH}_{2} \mathrm{CH}_{2} \mathrm{CH}=\mathrm{CH}_{2}\right), 4.41(\mathrm{t}, J=6.9 \mathrm{~Hz}, 2 \mathrm{H}$, $\left.\mathrm{NCH}_{2}\right), 2.71\left(\mathrm{~m}, 2 \mathrm{H}, \mathrm{CH}_{2}\right) .{ }^{13} \mathbf{C} \mathbf{N M R}\left(101 \mathrm{MHz}, \mathrm{CDCl}_{3}\right) \delta 166.8,137.9,136.7,133.6,133.4$, $133.2,132.5,130.5,129.4,125.2,124.2,122.5,120.0,118.6,116.2,110.9,79.0,46.9,34.2$. IR v 3082 (w), 1607 (s), 1557 (m), 1500 (m), 1456 (w), 1437 (w), 1389 (w), 1358 (m), 1262 (w), 1160 (w), 1005 (w), 919 (w), 830 (w). HRMS (ESI) calcd for $\mathrm{C}_{19} \mathrm{H}_{17} \mathrm{INO}_{2}{ }^{+}[\mathrm{M}+\mathrm{H}]^{+}$418.0299; found 418.0294. The structure of the obtained regioisomer was assigned by NMR correlation to compound $9 b$.

\section{1-(3-1-(2-((Triisopropylsilyl)oxy)ethyl)-1H-indole)-1 $H$-1 $\lambda_{3}$-benzo[b]iodo-3(2H)-one (9d)}

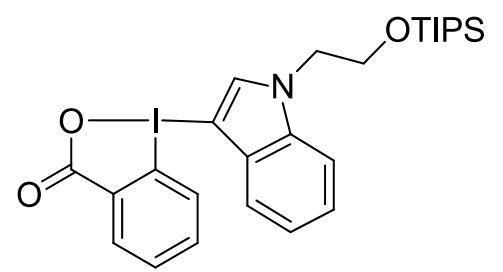

9d

Starting from 1-(2-((triisopropylsilyl)oxy)ethyl)-1H-indole $\mathbf{4 0}$ (318 $\mathrm{mg}, \quad 1.00 \mathrm{mmol})$, after 16 hours 1-(3-1-(2((triisopropylsilyl)oxy)ethyl)- $1 H$-indole)- $1 H-1 \lambda_{3} \quad$-benzo[b]iodo$3(2 H)$-one $9 d$ ( $873 \mathrm{mg}, 0.929 \mathrm{mmol}, 60 \%$ yield) was obtained as an orange oil. Rf: 0.40 (DCM:MeOH 9:1). ${ }^{1} \mathbf{H}$ NMR (400 MHz, $\left.\mathrm{CDCl}_{3}\right) \delta 8.47(\mathrm{~d}, J=7.5 \mathrm{~Hz}, 1 \mathrm{H}, \mathrm{Ar} H), 7.99$ (s, $\left.1 \mathrm{H}, \mathrm{NCHCI}\right), 7.65$ (d, $\left.J=8.5 \mathrm{~Hz}, 1 \mathrm{H}, \mathrm{Ar} H\right), 7.59$ $(\mathrm{t}, J=7.5 \mathrm{~Hz}, 1 \mathrm{H}, \operatorname{Ar} H), 7.51-7.45(\mathrm{~m}, 2 \mathrm{H}, \operatorname{Ar} H), 7.35(\mathrm{~m}, 2 \mathrm{H}, \operatorname{Ar} H), 6.94(\mathrm{~d}, J=8.3 \mathrm{~Hz}, 1 \mathrm{H}$, $\mathrm{ArH}), 4.54$ (t, $\left.J=5.0 \mathrm{~Hz}, 2 \mathrm{H}, \mathrm{CH}_{2}\right), 4.20$ (t, $\left.J=5.0 \mathrm{~Hz}, 2 \mathrm{H}, \mathrm{CH}_{2}\right), 1.09(\mathrm{dd}, J=8.7,5.6 \mathrm{~Hz}, 3 \mathrm{H}$, $\left.\mathrm{SiCH}\left(\mathrm{CH}_{3}\right)_{2}\right), 1.01\left(\mathrm{~d}, J=7.1 \mathrm{~Hz}, 18 \mathrm{H}, \mathrm{SiCH}\left(\mathrm{CH}_{3}\right)_{2}\right) .{ }^{13} \mathbf{C} \mathbf{N M R}\left(101 \mathrm{MHz}, \mathrm{CDCl}_{3}\right) \delta 167.0,138.8$, 
137.1, 133.2, 133.2, 132.4, 130.4, 129.1, 125.3, 124.0, 122.4, 119.7, 115.8, 111.0, 78.3, 62.3, 49.8, 17.7, 11.6. IR $v 2942(\mathrm{w}), 2890$ (w), 2865 (m), 1716 (w), 1604 (s), 1555 (w), 1500 (w), 1458 (w), 1358 (m), 1249 (m), 1169 (w), 1115 (w), 1015 (w), 883 (m), 831 (m). HRMS (ESI) calcd for $\mathrm{C}_{26} \mathrm{H}_{35} \mathrm{INO}_{3} \mathrm{Si}^{+}[\mathrm{M}+\mathrm{H}]^{+}$564.1425; found 564.1431. The structure of the obtained regioisomer was assigned by NMR correlation to compound $\mathbf{9 b}$.

\section{1-(3-1-(3-Phenylpropyl)-1 $H$-indole)-1 $H$-1 $1 \lambda_{3}$-benzo $[b]$ iodo-3(2H)-one (9e)}<smiles>O=C1OI(c2cn(CCCc3ccccc3)c3ccccc23)c2ccccc21</smiles>

$9 e$

Starting from 1-(3-phenylpropyl)--indole 41 (235 mg, $1.00 \mathrm{mmol})$, after 16 hours 1-(3-1-(3-phenylpropyl)-1H-indole)-1H-1 $\lambda_{3} \quad$ benzo[b]iodo-3(2H)-one 9e (436 mg, $0.906 \mathrm{mmol}$, 91\% yield) was obtained as an orange resin. Rf: 0.45 (DCM:MeOH 9:1). ${ }^{1} \mathbf{H}$ NMR $\left(400 \mathrm{MHz}, \mathrm{CDCl}_{3}\right) \delta 8.37(\mathrm{dd}, J=7.5,1.7 \mathrm{~Hz}, 1 \mathrm{H}, \mathrm{Ar} H), 7.86(\mathrm{~s}$, 1H, NCHCI), $7.54-7.36$ (m, 4H, ArH), $7.33-7.19$ (m, 5H, ArH), $7.18-7.14$ (m, 2H, ArH), 6.79 $(\mathrm{dd}, J=8.3,0.8 \mathrm{~Hz}, 1 \mathrm{H}, \mathrm{Ar} H), 4.34\left(\mathrm{t}, J=7.2 \mathrm{~Hz}, 2 \mathrm{H}, \mathrm{NCH}_{2}\right), 2.73\left(\mathrm{t}, J=7.5 \mathrm{~Hz}, 2 \mathrm{H}, \mathrm{CH}_{2} \mathrm{Ph}\right.$ ), $2.35\left(\mathrm{~m}, 2 \mathrm{H}, \mathrm{CH}_{2}-\mathrm{CH}_{2}-\mathrm{CH}_{2}\right) .{ }^{13} \mathbf{C}$ NMR $\left(101 \mathrm{MHz}, \mathrm{CDCl}_{3}\right) \delta 166.8,140.0,137.8,136.8,133.4$, 133.2, 132.4, 130.4, 129.3, 128.6, 128.3, 126.4, 125.2, 124.1, 122.5, 120.0, 116.1, 110.9, 78.9, 46.7, 32.8, 31.1. IR v 3060 (w), 3026 (w), 2939 (w), 1717 (w), 1601 (s), 1551 (m), 1498 (m), 1455 (w), 1364 (w), 1245 (w), 1165 (w), 1005 (w), 828 (m). HRMS (ESI) calcd for $\mathrm{C}_{24} \mathrm{H}_{21} \mathrm{INO}_{2}{ }^{+}[\mathrm{M}+\mathrm{H}]^{+} 482.0612$; found 482.0614 . The structure of the obtained regioisomer was assigned by NMR correlation to compound $\mathbf{9 b}$.

\section{1-(3-1,2-Dimethyl-1 $H$-indole)-1 $H$-1 $\lambda_{3}$-benzo $[b]$ iodo-3(2H)-one (9f)}<smiles>Cc1c(I2COC(=O)c3ccccc32)c2ccccc2n1C</smiles>

9f

Starting from 2-methyl-1-methyl- $1 H$-indole 28 (145 mg, $1.00 \mathrm{mmol})$, after 16 hours 1-(3-1,2-dimethyl- $1 H$-indole)-1H-1 $\lambda_{3}$-benzo[b]iodo-3(2H)-one 9f (364 mg, $0.930 \mathrm{mmol}, 93 \%$ yield) was obtained as a dark violet foam. Rf: 0.43 (DCM:MeOH 9:1). ${ }^{1} \mathbf{H}$ NMR $\left(400 \mathrm{MHz}, \mathrm{CDCl}_{3}\right) \delta 8.42(\mathrm{dd}, J=$ 7.3, $1.7 \mathrm{~Hz}, 1 \mathrm{H}, \operatorname{Ar} H), 7.52$ (td, $J=7.3,0.9 \mathrm{~Hz}, 1 \mathrm{H}, \operatorname{Ar} H), 7.44$ (d, $J=8.2$ $\mathrm{Hz}, 1 \mathrm{H}, \operatorname{Ar} H), 7.35$ (m, 2H, ArH), 7.28 (m, 1H, ArH), 7.23 (ddd, $J=8.2,6.9,1.0 \mathrm{~Hz}, 1 \mathrm{H}, \operatorname{Ar} H$ ), $6.77(\mathrm{~m}, 1 \mathrm{H}, \mathrm{ArH}), 3.91\left(\mathrm{~s}, 3 \mathrm{H}, \mathrm{CH}_{3} \mathrm{~N}\right), 2.65(\mathrm{~s}, 3 \mathrm{H}, \mathrm{ICH}=\mathrm{CHCH}) .{ }^{13} \mathbf{C} \mathbf{N M R}\left(101 \mathrm{MHz}, \mathrm{CDCl}_{3}\right)$ 
$\delta 166.8,145.3,137.9,133.7,133.2,132.7,130.5,128.9,124.7,123.6,122.4,119.2,115.5,110.4$, 80.1, 31.1, 13.2. IR $v 3055$ (w), $2987(\mathrm{w}), 2948$ (w), 1717 (w), 1605 (s), $1584(\mathrm{~m}), 1553$ (m), 1516 (w), $1472(\mathrm{w}), 1437$ (w), 1395 (m), 1378 (m), $1268(\mathrm{~m}), 1154$ (w), 1032 (w), $1011(\mathrm{w}), 829(\mathrm{w})$. HRMS (ESI) calcd for $\mathrm{C}_{17} \mathrm{H}_{15} \mathrm{INO}_{2}{ }^{+}[\mathrm{M}+\mathrm{H}]^{+}$392.0142; found 392.0146. The structure of the obtained regioisomer was assigned by NMR correlation to compound $\mathbf{9 b}$.

\section{1-(3-5-Methoxy-1-methyl-1H-indole)-1H-1 $\lambda_{3}$-benzo[b]iodo-3(2H)-one (9g)}<smiles>COc1ccc2c(c1)c(-c1ccccc1C(=O)O)cn2C</smiles>

$9 \mathrm{~g}$

Starting from 5-methoxy-1-methyl- $1 H$-indole 29 (161 mg, $1.00 \mathrm{mmol})$, after 16 hours 1-(3-5-methoxy-1-methyl-1 $H$-indole)- $1 H$ - $1 \lambda_{3}$-benzo[b]iodo$3(2 H)$-one $9 \mathrm{~g}$ (346 mg, $0.850 \mathrm{mmol}, 85 \%$ yield) was obtained as an orange resin. Rf: 0.42 (DCM:MeOH 9:1). NB: the reagent was isolated in presence of a small amount of the polymerized form of the heterocycles (4\% of estimated impurity). ${ }^{1} \mathrm{H}$ NMR (400 MHz, $\left.\mathrm{CDCl}_{3}\right) \delta 8.35(\mathrm{dd}, J=7.5,1.6 \mathrm{~Hz}, 1 \mathrm{H}, \mathrm{Ar} H$ ), 7.82 (s, 1H, NCHCI), 7.48 (t, $J=7.3 \mathrm{~Hz}, 1 \mathrm{H}, \operatorname{Ar} H), 7.40$ (d, $J=9.0 \mathrm{~Hz}, 1 \mathrm{H}, \operatorname{Ar} H), 7.27$ (m, 1H, $\operatorname{Ar} H), 7.04(\mathrm{dd}, J=9.0,2.4 \mathrm{~Hz}, 1 \mathrm{H}, \operatorname{Ar} H), 6.84(\mathrm{~d}, J=8.3 \mathrm{~Hz}, 1 \mathrm{H}, \operatorname{Ar} H), 6.79$ (d, $J=2.3 \mathrm{~Hz}, 1 \mathrm{H}$, $\mathrm{ArH}), 3.99\left(\mathrm{~s}, 3 \mathrm{H}, \mathrm{CH}_{3} \mathrm{~N}\right), 3.78\left(\mathrm{~s}, 3 \mathrm{H}, \mathrm{OCH}_{3}\right) .{ }^{13} \mathbf{C} \mathbf{N M R}\left(101 \mathrm{MHz}, \mathrm{CDCl}_{3}\right) \delta 167.1,156.4,138.9$, $133.3,132.5,132.5,130.5,130.1,125.2,116.0,114.8,111.7,100.8,77.4,55.8,34.0$ (the signal for one aromatic C could not be resolved). IR $v 3110$ (w), 3062 (w), 3000 (w), 2944 (w), 2837 (w), 1719 (w), 1604 (m), 1584 (m), 1556 (w), 1504 (m), 1489 (m), 1439 (w), 1378 (w), 1249 (s), 1223 (s), $1158(\mathrm{~m}), 1031$ (s), $970(\mathrm{w}), 845$ (m). HRMS (ESI) calcd for $\mathrm{C}_{17} \mathrm{H}_{15} \mathrm{INO}_{3}{ }^{+}[\mathrm{M}+\mathrm{H}]^{+} 408.0091$; found 408.0093. The structure of the obtained regioisomer was assigned by NMR correlation to compound $9 b$.

\section{1-(3-5-Fluoro-1-methyl-1H-indole)-1H-1 $\lambda_{3}$-benzo[b]iodo-3(2H)-one (9h)}<smiles>Cn1cc(I)c2cc(F)ccc21</smiles>

$9 \mathrm{~h}$

Starting from 5-fluoro-1-methyl-1 $H$-indole 30 (149 mg, $1.00 \mathrm{mmol})$, after 16 hours 1-(3-5-fluoro-1-methyl-1 $H$-indole)- $1 H$ - $1 \lambda_{3}$-benzo[b]iodo-3(2H)one $9 \mathrm{~h}$ (336 mg, $0.850 \mathrm{mmol}, 85 \%$ yield) was obtained as an yellow foam. Rf: 0.3 (DCM:MeOH 9:1). NB: the reagent was isolated in presence of a small amount of the polymerized form of the heterocycles $(7 \%$ of 
estimated impurity). ${ }^{1} \mathrm{H}$ NMR $\left(400 \mathrm{MHz}, \mathrm{CD}_{3} \mathrm{OD}\right) \delta 8.1(\mathrm{dd}, J=7.6,1.7 \mathrm{~Hz}, 1 \mathrm{H}, \mathrm{Ar} H), 8.01$ (s, 1H, NCHCI), 7.57 (ddd, $J=8.8,4.2,0.8 \mathrm{~Hz}, 1 \mathrm{H}, \operatorname{Ar} H), 7.49$ (td, $J=7.4,1.0 \mathrm{~Hz}, 1 \mathrm{H}, \operatorname{Ar} H), 7.30$ (ddd, $J=8.3,7.2,1.7 \mathrm{~Hz}, 1 \mathrm{H}, \operatorname{Ar} H), 7.14-7.03$ (m, 2H, ArH), 6.80 (dd, $J=8.3,0.9 \mathrm{~Hz}, 1 \mathrm{H}, \operatorname{Ar} H$ ), $3.93\left(\mathrm{~s}, 3 \mathrm{H}, \mathrm{CH}_{3} \mathrm{~N}\right) .{ }^{13} \mathrm{C}$ NMR $\left(101 \mathrm{MHz}, \mathrm{CD}_{3} \mathrm{OD}\right) \delta 170.3,160.91(\mathrm{~d}, J=238.2 \mathrm{~Hz}), 142.6,135.9$, 135.1, 134.4, 133.2, 131.7, 131.40 (d, $J=10.7 \mathrm{~Hz}), 127.7,116.8,113.71$ (d, $J=9.8 \mathrm{~Hz}), 113.47$ $(\mathrm{d}, J=26.7 \mathrm{~Hz}), 105.67(\mathrm{~d}, J=25.3 \mathrm{~Hz}), 77.5,34.3 .{ }^{19} \mathbf{F} \mathbf{N M R}\left(376 \mathrm{MHz}, \mathrm{CDCl}_{3}\right) \delta-119.8$. IR $v$ 3083 (w), 2952 (w), 1717 (w), 1601 (s), 1557 (m), 1503 (m), 1485 (m), 1438 (w), 1338 (m), 1242 (m), 1193 (m), 1121 (m), 1032 (w), 1005 (w), 850 (m). HRMS (ESI) calcd for $\mathrm{C}_{16} \mathrm{H}_{12} \mathrm{FINO}_{2}{ }^{+}[\mathrm{M}+\mathrm{H}]^{+}$395.9891; found 395.9894. The structure of the obtained regioisomer was assigned by NMR correlation to compound $\mathbf{9 b}$.

\section{1-(3-5-Chloro-1-methyl-1H-indole)-1H-1 $\lambda_{3}$-benzo[b]iodo-3(2H)-one (9i)}<smiles>Cn1cc(I)c2cc(Cl)ccc21</smiles>

$9 \mathbf{i}$

Starting from 5-chloro-1-methyl-1 $H$-indole 31 (166 mg, $1.00 \mathrm{mmol})$, after 16 hours 1-(3-5-chloro-1-methyl-1H-indole)-1H-1 $\lambda_{3}$-benzo[b]iodo-3(2H)one 9i (256 mg, $0.622 \mathrm{mmol}, 62 \%$ yield) was obtained as an orange amorphous solid. Rf: 0.4 (DCM:MeOH 9:1). ${ }^{1} \mathbf{H}$ NMR (400 MHz, $\mathrm{CD}_{2} \mathrm{Cl}_{2}$ ) $\delta 8.20(\mathrm{~d}, J=7.4 \mathrm{~Hz}, 1 \mathrm{H}, \operatorname{Ar} H), 7.78(\mathrm{~s}, 1 \mathrm{H}, \mathrm{NCHCI}), 7.38(\mathrm{~d}, J=8.7 \mathrm{~Hz}$, 1H, $\operatorname{Ar} H), 7.34-7.22$ (m, 3H, ArH), 7.17 (t, $J=7.7 \mathrm{~Hz}, 1 \mathrm{H}, \operatorname{Ar} H), 6.68$ (d, $J=8.2 \mathrm{~Hz}, 1 \mathrm{H}, \operatorname{Ar} H$ ), 3.87 (s, 3H, $\left.\mathrm{CH}_{3} \mathrm{NCHCI}\right) .{ }^{13} \mathrm{C}$ NMR $\left(101 \mathrm{MHz}, \mathrm{CD}_{3} \mathrm{OD}\right) \delta 170.3,142.5,137.8,135.1,134.4$, 133.2, 131.7, 131.7, 129.6, 127.8, 125.4, 120.0, 116.8, 113.7, 77.5, 34.2 IR $v 3095$ (w), 2953 (w), 1605 (s), 1556 (m), 1505 (m), 1438 (m), 1388 (m), 1370 (m), 1260 (s), 1226 (m), 1164 (m), 1144 (m), 1115 (w), 1070 (w), 1032 (s), 1004 (w), 837 (w). HRMS (ESI) calcd for $\mathrm{C}_{16} \mathrm{H}_{12} \mathrm{ClINO}_{2}{ }^{+}[\mathrm{M}+\mathrm{H}]^{+}$411.9596; found 411.9603. The structure of the obtained regioisomer was assigned by NMR correlation to compound $\mathbf{9 b}$.

1-(3-5-Iodo-1-methyl-1 $H$-indole)-1 $H$-1 $1 \lambda_{3}$-benzo $[b]$ iodo-3(2H)-one (9j) 
<smiles>Cn1cc(I)c2cc(I)ccc21</smiles>

9j

Starting from 5-iodo-1-methyl- $1 H$-indole 32 (257 mg, $1.00 \mathrm{mmol})$, after 16 hours 1-(3-5-iodo-1-methyl-1H-indole)-1H-1 $\lambda_{3}$-benzo[b]iodo-3(2H)-one 9j (380 mg, $0.755 \mathrm{mmol}, 76 \%$ yield) was obtained as a yellow amorphous solid. Rf: 0.3 (DCM:MeOH 9:1). NB: the reagent is unstable in acidic deuterated solvents and it decompose in short time, we recommend the immediate use after the synthesis. The proton NMR presents about $21 \%$ of the open protonated form. ${ }^{1} \mathbf{H}$ NMR (400 MHz, $\left.\mathrm{CDCl}_{3}\right) \delta 8.24(\mathrm{~m}, 1 \mathrm{H}, \mathrm{ArH}), 7.83$ (s, 1H, NCHCI), $7.58-7.47$ (m, 2H, $\operatorname{Ar} H), 7.26\left(\mathrm{~m}, 1 \mathrm{H}, \mathrm{Ar} H+\mathrm{CDCl}_{3}\right), 7.21-7.18(\mathrm{~m}, 2 \mathrm{H}, \mathrm{Ar} H), 6.67(\mathrm{~d}, J=8.1 \mathrm{~Hz}, 1 \mathrm{H}, \mathrm{ArH}), 3.87$ $\left(\mathrm{s}, 3 \mathrm{H}, \mathrm{CH}_{3} \mathrm{~N}\right) .{ }^{13} \mathbf{C}$ NMR $\left(101 \mathrm{MHz}, \mathrm{CDCl}_{3}\right) \delta 168.6,140.5,136.8,133.7,132.6,132.3,131.4$, 130.3, 128.1, 126.0, 122.3, 119.1, 115.7, 112.9, 86.1, 34.0. IR $v 3092$ (w), 3061 (w), 1600 (s), 1584 (m), 1557 (m), 1503 (m), 1436 (w), 1422 (w), 1371 (m), 1265 (s), 1245 (s), 1225 (m), 1163 (m), 1113 (w), 1031 (s), 1004 (w), 836 (w). HRMS (ESI) calcd for $\mathrm{C}_{16} \mathrm{H}_{12} \mathrm{I}_{2} \mathrm{NO}_{2}{ }^{+}[\mathrm{M}+\mathrm{H}]^{+}$503.8952; found 503.8952. The structure of the obtained regioisomer was assigned by NMR correlation to compound $9 b$.

\section{1-(3-1-Methyl-5-(4,4,5,5-tetramethyl-1,3,2-dioxaborolan-2-yl)-1 $H$-indole)-1 $H$ - $1 \lambda_{3}$ - benzo[b]iodo-3(2H)-one (9k)}<smiles>Cn1cc(I)c2cc(Br)ccc21</smiles>

$9 k$

Starting from commercially available 1-methyl-5-(4,4,5,5-tetramethyl1,3,2-dioxaborolan-2-yl)-1H-indole (149 mg, $1.00 \mathrm{mmol})$, after 16 hours 1 (3-1-methyl-5-(4,4,5,5-tetramethyl-1,3,2-dioxaborolan-2-yl)-1H-indole)$1 H$ - $1 \lambda_{3}$-benzo[ $\left.b\right]$ iodo-3(2H)-one $9 k$ ( $276 \mathrm{mg}, 0.549 \mathrm{mmol}, 54 \%$ yield) was obtained as an orange amorphous solid. Rf: 0.46 (DCM:MeOH 9:1). ${ }^{1} \mathbf{H}$ NMR $\left(400 \mathrm{MHz}, \mathrm{CD}_{2} \mathrm{Cl}_{2}\right) \delta 8.32(\mathrm{dd}, J=7.5,1.7 \mathrm{~Hz}, 1 \mathrm{H}, \mathrm{Ar} H), 7.93$ (s, 1H, NCHCI), 7.81 (dd, $J$ = 8.4, 1.1 Hz, 1H, ArH), 7.74 (s, 1H, CCHCBPin), $7.59-7.48(\mathrm{~m}, 2 \mathrm{H}, \operatorname{Ar} H), 7.31$ (ddd, $J=8.6$, 7.1, $1.7 \mathrm{~Hz}, 1 \mathrm{H}, \operatorname{Ar} H), 6.85(\mathrm{dd}, J=8.3,0.9 \mathrm{~Hz}, 1 \mathrm{H}, \operatorname{Ar} H), 3.99\left(\mathrm{~s}, 3 \mathrm{H}, \mathrm{CH}_{3} \mathrm{~N}\right), 1.30(\mathrm{~s}, 12 \mathrm{H}$, CBPin). ${ }^{13} \mathbf{C}$ NMR (101 MHz, $\left.\mathrm{CD}_{2} \mathrm{Cl}_{2}\right) \delta 167.0,140.1,139.6,134.1,133.8,132.6,131.0,130.5$, 129.5, 127.6, 126.0, 117.1, 110.7, 84.5, 80.3, 34.4, 25.2 (one aromatic Carbon signal not resolved). IR $v 3095$ (w), 2979 (w), 1611 (s), 1558 (w), 1507 (w), 1436 (w), 1360 (s), 1303 (w), 1263 (w), 1142 (s), 1114 (w), 1074 (w), 970 (w), 861 (w). HRMS (ESI) calcd for 
$\mathrm{C}_{22} \mathrm{H}_{24} \mathrm{BINO}_{4}{ }^{+}[\mathrm{M}+\mathrm{H}]^{+}$504.0838; found 504.0835. The structure of the obtained regioisomer was assigned by NMR correlation to compound $\mathbf{9 b}$.

\section{1-(3-1-Methyl-1H-pyrrolo[2,3-b]pyridine)-1H-1 $\lambda_{3}$-benzo[b]iodo-3(2H)-one (9l)}<smiles>Cn1cc(I2COC(=O)c3ccccc32)c2cccnc21</smiles>

9|

Starting from commercially available 1-methyl-1H-pyrrolo[2,3-b]pyridine (132 mg, $1.00 \mathrm{mmol})$, after 16 hours 1-(3-1-methyl-1H-pyrrolo[2,3$b]$ pyridine)-1 $H$ - $1 \lambda_{3}$-benzo[b]iodo-3(2H)-one 91 (114 mg, $0.301 \mathrm{mmol}, 30 \%$ yield) was obtained as a brown amorphous solid. Rf: 0.2 (DCM:MeOH 9:1). ${ }^{1} \mathbf{H}$ NMR $\left(400 \mathrm{MHz}, \mathrm{CD}_{2} \mathrm{Cl}_{2}\right) \delta 8.37(\mathrm{dd}, J=4.7,1.6 \mathrm{~Hz}, 1 \mathrm{H}, \mathrm{Ar} H), 8.06$ (dd, $J=7.9,1.2 \mathrm{~Hz}, 1 \mathrm{H}, \operatorname{Ar} H), 7.96(\mathrm{dd}, J=7.9,1.7 \mathrm{~Hz}, 1 \mathrm{H}, \operatorname{Ar} H), 7.92(\mathrm{dd}, J=7.9,1.7 \mathrm{~Hz}, 1 \mathrm{H}$, $\operatorname{Ar} H), 7.46(\mathrm{td}, J=7.7,1.2 \mathrm{~Hz}, 1 \mathrm{H}, \operatorname{Ar} H), 7.25-7.19(\mathrm{~m}, 2 \mathrm{H}, \operatorname{Ar} H), 7.14(\mathrm{dd}, J=7.9,4.7 \mathrm{~Hz}, 1 \mathrm{H}$, $\mathrm{ArH}), 3.86\left(\mathrm{~s}, 3 \mathrm{H}, \mathrm{CH}_{3} \mathrm{~N}\right) .{ }^{13} \mathrm{C}$ NMR $\left(101 \mathrm{MHz}, \mathrm{CD}_{2} \mathrm{Cl} 2\right) \delta 168.6,144.4,142.3,134.3,133.8$, 132.1, 128.7, 127.0, 126.3, 118.8, 116.5, 103.2, 94.9, 31.7 (one Carbon signal not resolved). IR v 2925 (w), 2852 (w), 1719 (s), 1599 (m), 1585 (m), 1468 (m), 1408 (w), 1348 (w), 1289 (m), 1262 (s), $1136(\mathrm{~m}), 1103$ (w), 1042 (w), 1017 (m), 976 (w). HRMS (ESI) calcd for $\mathrm{C}_{15} \mathrm{H}_{12} \mathrm{IN}_{2} \mathrm{O}_{2}{ }^{+}[\mathrm{M}+\mathrm{H}]^{+}$378.9938; found 378.9945. The structure of the obtained regioisomer was assigned by NMR correlation to compound $\mathbf{9 b}$.

\section{1-(3-1H-Pyrrole)-1H-1 $1 \lambda_{3}$-benzo $[b]$ iodo-3(2H)-one (9m)}<smiles>O=C1OI(c2cc[nH]c2)c2ccccc21</smiles>

$9 \mathrm{~m}$

Starting from 1-(tert-butyldimethylsilyl)-1 $H$-pyrrole $35(181 \mathrm{mg}, 1.00 \mathrm{mmol})$ and using $\mathrm{Sc}(\mathrm{OTf})_{3}$ as the Lewis Acid (20 mol\%), after 16 hours 1-(3-1Hpyrrole)- $1 H-1 \lambda_{3}$-benzo[b]iodo-3(2H)-one 9m (225 mg, $0.719 \mathrm{mmol}, 72 \%$ yield) was obtained as a slightly brown solid. Rf: 0.5 (DCM:MeOH 9:1). Mp: $147^{\circ} \mathrm{C}$ (decomposition). ${ }^{1} \mathbf{H}$ NMR $\left(400 \mathrm{MHz}, \mathrm{CD}_{3} \mathrm{OD}\right) \delta 8.40(\mathrm{dd}, J=7.5,1.7 \mathrm{~Hz}, 1 \mathrm{H}, \mathrm{ArH}), 7.72$ $(\mathrm{d}, J=7.4 \mathrm{~Hz}, 1 \mathrm{H}, \operatorname{Ar} H), 7.68-7.59$ (m, 2H, ArH), 7.23 (t, $J=2.4 \mathrm{~Hz}, 1 \mathrm{H}, \operatorname{Ar} H), 7.14(\mathrm{~d}, J=8.2$ $\mathrm{Hz}, 1 \mathrm{H}, \mathrm{ArH}), 6.74(\mathrm{dd}, J=2.8,1.5 \mathrm{~Hz}, 1 \mathrm{H}, \mathrm{ArH})\left(\mathrm{NH}\right.$ signal exchanges with $\left.\mathrm{CD}_{3} \mathrm{OD}\right) .{ }^{13} \mathbf{C} \mathbf{~ N M R}$ (101 MHz, $\left.\mathrm{CD}_{3} \mathrm{OD}\right) \delta 170.3,135.0,134.4,132.9,131.5,130.4,128.0,123.7,117.3,115.9,83.4$. IR $v 3484(w), 1607$ (w), 1558 (w), 1439 (w), 1397 (w), 1260 (s), 1236 (s), 1174 (s), 1084 (w), 1050 (m), 1038 (m), 903 (w), 882 (w). HRMS (ESI) calcd for $\mathrm{C}_{11} \mathrm{H}_{9} \mathrm{INO}_{2}{ }^{+}[\mathrm{M}+\mathrm{H}]^{+}$313.9673; found 313.9673. The structure of the reagent was assigned based on X-Ray diffraction (CCDC number 1541174). 


\section{1-(3-1-Methyl-1H-pyrrole)-1 $H-1 \lambda_{3}$-benzo $[b]$ iodo-3(2H)-one (9n) and 1-(2-1-methyl-1H- pyrrole)-1 $H-1 \lambda_{3}$-benzo[b]iodo-3(2H)-one (9o)}<smiles>Cn1ccc(I2COC(=O)c3ccccc32)c1</smiles>

9n<smiles>Cn1cccc1I1C(=O)c2ccccc21</smiles>

90

Starting from commercially available 1-methyl- $1 H$ pyrrole $(0.890 \mathrm{ml}, 1.00 \mathrm{mmol})$, after 12 hours 1-(3-1methyl-1 $H$-pyrrole)- $1 H-1 \lambda_{3} \quad$-benzo[b]iodo-3(2H)-one 9n and 1-(2-1-methyl-1H-pyrrole)-1H-1 $\lambda_{3}$ benzo[b]iodo-3(2H)-one 9o were obtained as a $1: 1$ mixture (overall yield 95\%) as an off-white, sticky amorphous solid. Rf: 0.5 (DCM:MeOH 9:1). The two compounds were separated by slow flash column chromatography (EtOAc:MeOH 9:1). The structure of the obtained regioisomers were assigned by NMR correlation to compound $\mathbf{9 m}$.<smiles>Cn1ccc(I2COC(=O)c3ccccc32)c1</smiles>

9n

1-(3-1-methyl-1H-pyrrole)-1H-1 $\lambda_{3}$-benzo[b]iodo-3(2H)-one 9n (158 mg, 0.483 mmol, 48\% yield; off-white, sticky amorphous solid). Rf: 0.25 (EtOAc:MeOH 9:1). ${ }^{1} \mathbf{H}$ NMR (400 MHz, CD $\left.{ }_{3} \mathrm{OD}\right) \delta 8.15$ (dd, $J=7.5,1.7$ $\mathrm{Hz}, 1 \mathrm{H}, \mathrm{Ar} H), 7.53(\mathrm{td}, J=7.3,1.1 \mathrm{~Hz}, 1 \mathrm{H}, \mathrm{Ar} H), 7.49-7.40$ (m, 2H, ArH), $7.04-6.98$ (m, 2H, ArH), 6.58 (d, J=1.2 Hz, 1H, ArH), 3.85 (s, 3H, NMe). ${ }^{13}$ C NMR (101 MHz, $\left.\mathrm{CD}_{3} \mathrm{OD}\right) \delta 170.0,134.9,134.2,133.4,132.8,131.5,127.9,127.7,117.4$, 116.6, 82.8, 37.1. IR $v 3447$ (w), 3106 (w), 2947 (w), 2863 (w), 1603 (s), 1591 (m), 1558 (m), 1512 (m), 1437 (w), 1354 (m), 1294 (w), 1110 (m), 1083 (w), 1007 (w), 829 (m). HRMS (ESI) calcd for $\mathrm{C}_{12} \mathrm{H}_{11} \mathrm{INO}_{2}{ }^{+}[\mathrm{M}+\mathrm{H}]^{+}$327.9829; found 327.9831. DSC-analysis was performed on compound 9n: see par.5 of Supplementary informations.<smiles>Cn1cccc1I1C(=O)c2ccccc21</smiles>

$\mathrm{Hz}, 1 \mathrm{H}, \mathrm{ArH}), 6.43(\mathrm{dd}, J=3.9,2.1 \mathrm{~Hz}, 1 \mathrm{H}, \mathrm{ArH}), 3.78\left(\mathrm{~s}, 1 \mathrm{H}, \mathrm{NCH}_{3}\right) .{ }^{13} \mathbf{C} \mathbf{N M R}(101 \mathrm{MHz}$, $\left.\mathrm{CD}_{3} \mathrm{OD}\right) \delta 170.1,135.5,134.2,133.2,131.9,131.4,127.7,126.6,119.4,112.9,96.0,37.4$. IR $v$ 
$3415(\mathrm{w}), 3105(\mathrm{w}), 3049(\mathrm{w}), 2950(\mathrm{w}), 1604(\mathrm{~s}), 1584(\mathrm{~m}), 1558(\mathrm{w}), 1508(\mathrm{w}), 1437(\mathrm{w}), 1346$ (m), 1288 (m), 1223 (w), 1149 (w), 1091 (w), 1047 (w), 1005 (w), 829 (m). HRMS (ESI) calcd for $\mathrm{C}_{12} \mathrm{H}_{11} \mathrm{INO}_{2}{ }^{+}[\mathrm{M}+\mathrm{H}]^{+}$327.9829; found 327.9842. DSC-analysis was performed on compound 9o: see par.5 of Supplementary informations.

\section{1-(3-1-benzyl-1H-pyrrole)-1H-1 $\lambda_{3}$-benzo[b]iodo-3(2H)-one $(9 \mathrm{p})$ and 1-(2-1-benzyl-1H- pyrrole)-1H-1 $\lambda_{3}$-benzo[b]iodo-3(2H)-one (9q)}
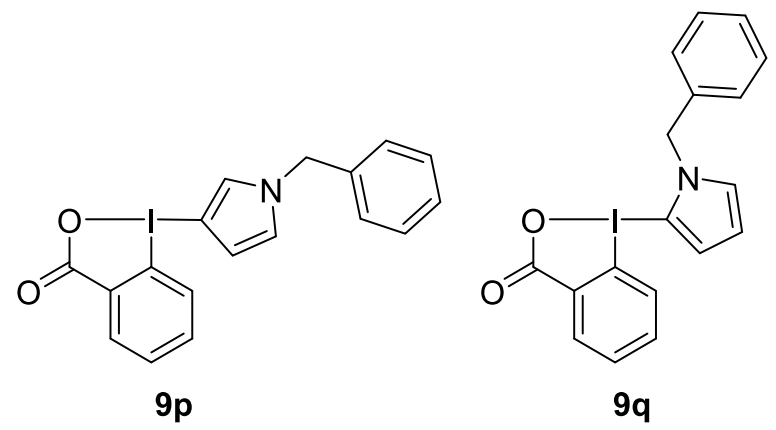

Starting from commercially available 1 -methyl$1 H$-pyrrole $(0.890 \mathrm{ml}, 1.00 \mathrm{mmol})$, after 12 hours 1-(3-1-benzyl-1H-pyrrole)- $1 H-1 \lambda_{3}$ benzo[b]iodo-3(2H)-one 9p and 1-(2-1-benzyl$1 H$-pyrrole)- $1 H$ - $1 \lambda_{3}$-benzo[ $\left.b\right]$ iodo-3(2H)-one $\mathbf{9 q}$ were obtained in 3:1 mixture (overall yield $86 \%$ ), as a colorless amorphous solid. Rf: 0.7

(DCM:MeOH 9:1). The two compounds were separated by slow flash column chromatography (EtOAc:MeOH 9:1). The structure of the obtained regioisomers were assigned by NMR correlation to compound $\mathbf{9 m}$.

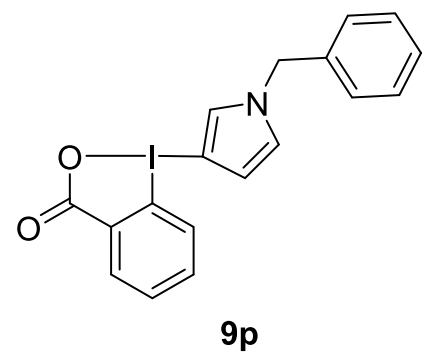

1-(3-1-benzyl-1H-pyrrole)-1H-1 $\lambda_{3}$-benzo[b]iodo-3(2H)-one 9p (260 $\mathrm{mg}, \quad 0.645 \mathrm{mmol}, 65 \%$ yield) as a colorless foam. Rf: 0.46 (EtOAc:MeOH 9:1). ${ }^{1} \mathbf{H}$ NMR (400 MHz, 2:1 mixture $C_{3} O D: C_{6} D_{6}$, refered to $\left.\mathrm{CD}_{3} \mathrm{OD}\right) \delta 8.29(\mathrm{dd}, J=7.5,1.7 \mathrm{~Hz}, 1 \mathrm{H}, \mathrm{Ar} H), 7.36(\mathrm{t}, J=$ $7.3 \mathrm{~Hz}, 1 \mathrm{H}, \mathrm{Ar} H), 7.30-7.20\left(\mathrm{~m}, 3 \mathrm{H} \mathrm{ArH}+\mathrm{C}_{6} \mathrm{D}_{6}\right), 7.18-7.08(\mathrm{~m}$, $3 \mathrm{H}, \operatorname{Ar} H), 6.97(\mathrm{~d}, J=2.0 \mathrm{~Hz}, 1 \mathrm{H}, \operatorname{Ar} H), 6.83-6.78$ (m, 2H, $\operatorname{Ar} H)$, $6.30(\mathrm{dd}, J=3.0,1.7 \mathrm{~Hz}, 1 \mathrm{H}, \mathrm{ArH}), 4.93\left(\mathrm{~s}, 2 \mathrm{H}, \mathrm{NCH}_{2} \mathrm{Ph}\right) .{ }^{13} \mathrm{C}$ NMR (101 MHz, 2:1 mixture $C D_{3} O D: C_{6} D_{6}$, refered to $\left.C D_{3} O D\right) \delta 168.7,136.8,133.5,133.0,131.8,130.9,130.3,128.8,128.1$, 127.4, 126.2, 125.5, 116.3, 115.3, 82.4, 53.5. IR v 3409 (w), 3114 (w), $2971(w), 1609$ (s), 1585 (m), $1558(\mathrm{w}), 1456(\mathrm{w}), 1438(\mathrm{w}), 1365$ (m), 1277 (s), 1160 (w), 1079 (w), 1032 (m), $835(\mathrm{w})$. HRMS (ESI) calcd for $\mathrm{C}_{18} \mathrm{H}_{15} \mathrm{INO}_{2}^{+}[\mathrm{M}+\mathrm{H}]^{+}$404.0142; found 404.0140. 


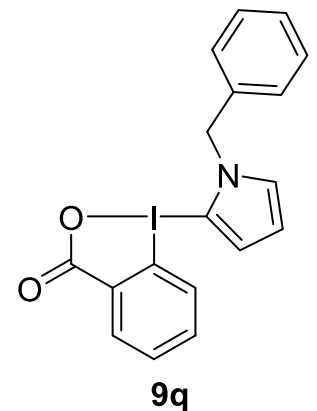

1-(2-1-benzyl-1H-pyrrole)-1H-1 $1 \lambda_{3}$-benzo[b]iodo-3(2H)-one 9q (85.0 mg, $0.211 \mathrm{mmol}, 21 \%$ yield) as a colorless foam. Rf: 0.5 (EtOAc:MeOH 9:1). ${ }^{1} \mathbf{H}$ NMR $\left(400 \mathrm{MHz}, 2: 1\right.$ mixture $C D_{3} O D: C_{6} D_{6}$, referered to $\left.C D_{3} O D\right) \delta 8.18(\mathrm{dd}$, $J=7.5,1.6 \mathrm{~Hz}, 1 \mathrm{H}, \operatorname{Ar} H), 7.28(\mathrm{t}, J=7.4 \mathrm{~Hz}, 1 \mathrm{H}, \operatorname{Ar} H), 7.10(\mathrm{t}, J=2.2 \mathrm{~Hz}$, $1 \mathrm{H}, \operatorname{Ar} H), 6.92\left(\mathrm{~m}, J=7.6,2.9 \mathrm{~Hz}, 6 \mathrm{H}, \mathrm{Ar} H+\mathrm{C}_{6} \mathrm{D}_{6}\right), 6.71(\mathrm{dd}, J=3.9,1.7$ $\mathrm{Hz}, 1 \mathrm{H}, \operatorname{Ar} H), 6.35$ (t, $J=3.4 \mathrm{~Hz}, 1 \mathrm{H}, \operatorname{Ar} H), 6.23$ (d, $J=8.3 \mathrm{~Hz}, 1 \mathrm{H}, \operatorname{Ar} H)$, $4.90\left(\mathrm{~s}, 2 \mathrm{H}, \mathrm{NCH} \mathrm{H}_{2} \mathrm{Ph}\right) .{ }^{13} \mathrm{C}$ NMR $\left(101 \mathrm{MHz}, 2: 1\right.$ mixture $\mathrm{CD}_{3} \mathrm{OD}: \mathrm{C}_{6} D_{6}$, referered to $\left.\mathrm{CD}_{3} \mathrm{OD}\right) \delta$ 169.8, 136.9, 134.6, 132.7, 131.2, 131.1, 129.5, 129.0, 128.5, 127.2, 119.2, 112.9, 94.8, 54.7 (two Carbon signals under the deuterated benzene). IR $v 3109$ (w), 3064 (w), 2968 (w), 2875 (w), 1609 (s), 1585 (m), 1558 (w), 1503 (w), 1456 (w), 1440 (w), 1357 (m), 1277 (w), 1103 (m), 1079 (w), 1032 (w), 1031 (w), 830 (w). HRMS (ESI) calcd for $\mathrm{C}_{18} \mathrm{H}_{15} \mathrm{INO}_{2}{ }^{+}[\mathrm{M}+\mathrm{H}]^{+}$404.0142; found 404.0140 .

\section{1-(3-9-Methyl-9H-carbazole)-1H-1 $\lambda_{3}$-benzo $[b]$ iodo-3(2H)-one (9r)}<smiles>Cn1c2ccccc2c2cc(I3COC(=O)c4ccccc43)ccc21</smiles>

$9 \mathrm{r}$

Me Starting from commercially available 9-methyl-9H-carbazole $(181 \mathrm{mg}$, $1.00 \mathrm{mmol})$, after 16 hours 1-(3-9-methyl-9H-carbazole)-1H-1 $\lambda_{3}$ benzo[b]iodo-3(2H)-one 9r (110 $\mathrm{mg}, 0.257 \mathrm{mmol}, 33 \%$ yield) was obtained as a grey amorphous solid. Rf: 0.2 (DCM:MeOH 9:1). ${ }^{1} \mathbf{H}$ NMR (400 MHz, $\left.\mathrm{CDCl}_{3}\right) \delta 8.67(\mathrm{~d}, J=1.6 \mathrm{~Hz}, 1 \mathrm{H}, \mathrm{Ar} H), 8.47$ (dd, $J=7.5,1.7 \mathrm{~Hz}, 1 \mathrm{H}, \mathrm{Ar} H$ ), $8.14(\mathrm{~d}, J=7.9 \mathrm{~Hz}, 1 \mathrm{H}, \operatorname{Ar} H), 7.96(\mathrm{dd}, J=8.5,1.6 \mathrm{~Hz}, 1 \mathrm{H}, \mathrm{Ar} H), 7.63(\mathrm{ddd}, J=8.4,7.1,1.2 \mathrm{~Hz}$, $1 \mathrm{H}, \operatorname{Ar} H), 7.59$ (d, $J=8.5 \mathrm{~Hz}, 1 \mathrm{H}, \operatorname{Ar} H), 7.57-7.52$ (m, 2H, ArH), 7.37 (td, $J=7.5,1.2 \mathrm{~Hz}, 1 \mathrm{H}$, $\operatorname{Ar} H), 7.30$ (ddd, $J=8.5,7.1,1.7 \mathrm{~Hz}, 1 \mathrm{H}, \operatorname{Ar} H), 6.69$ (dd, $J=8.5,0.9 \mathrm{~Hz}, 1 \mathrm{H}, \operatorname{Ar} H), 3.98$ (s, 3H, $\left.\mathrm{NCH}_{3}\right) .{ }^{13} \mathrm{C}$ NMR $\left(101 \mathrm{MHz}, \mathrm{CDCl}_{3}\right) \delta$ 166.5, 142.4, 141.4, 133.4, 133.3, 132.7, 130.6, 130.0, 127.7, 125.8, 125.7, 121.4, 120.8, 120.7, 116.3, 112.0, 109.3, 101.8, 53.4, 29.5. IR v $3082(\mathrm{w})$, 2974 (w), 2924 (w), 2816 (w), 1652 (s), 1588 (w), 1570 (w), 1456 (w), 1440 (m), 1295 (m), 1245 (w), 1139 (w), 1017 (w), $982(\mathrm{~m}), 831$ (w). HRMS (ESI) calcd for $\mathrm{C}_{20} \mathrm{H}_{15} \mathrm{INO}_{2}{ }^{+}[\mathrm{M}+\mathrm{H}]^{+}$428.0142; found 428.0147 .

1-(2-1H-Thiophene)-1H-1 $\lambda_{3}$-benzo $[b]$ iodo-3(2H)-one (9s) 
<smiles>O=C1OI(c2cccs2)c2ccccc21</smiles>

9s

Starting from commercially available thiophene $(80.0 \mu \mathrm{L}, 1.00 \mathrm{mmol})$ and using $\mathrm{Sc}(\mathrm{OTf})_{3}$ as the Lewis Acid (20 mol\%), after 16 hours 1-(2-1H-

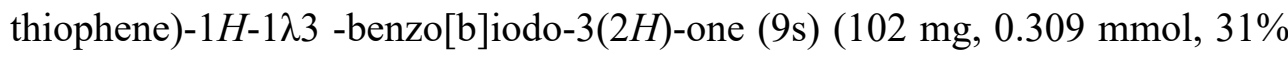
yield) was obtained as a light yellow amorphous solid. Rf: 0.4 (DCM:MeOH

9:1). NB: the reagent is unstable in protic deuterated solvents and it decompose in short time, we recommend the immediate use after the synthesis. The proton NMR presents about $7 \%$ of the open protonated form. ${ }^{1} \mathrm{H}$ NMR ${ }^{1} \mathrm{H}$ NMR (400 MHz, $\left.\mathrm{CD}_{3} \mathrm{OD}\right) \delta 8.28(\mathrm{dd}, J=7.5,1.7 \mathrm{~Hz}, 1 \mathrm{H}$, $\operatorname{Ar} H), 8.05(\mathrm{dd}, J=5.2,1.2 \mathrm{~Hz}, 1 \mathrm{H}, \operatorname{Ar} H), 7.98(\mathrm{dd}, J=3.7,1.2 \mathrm{~Hz}, 1 \mathrm{H}, \operatorname{Ar} H), 7.70(\mathrm{td}, J=7.4$, $0.9 \mathrm{~Hz}, 1 \mathrm{H}, \mathrm{Ar} H$ ), 7.61 (ddd, $J=8.7,7.2,1.7 \mathrm{~Hz}, 1 \mathrm{H}, \operatorname{Ar} H), 7.37$ (dd, $J=5.3,3.6 \mathrm{~Hz}, 1 \mathrm{H}, \operatorname{Ar} H$ ), $6.92(\mathrm{dd}, J=8.3,0.9 \mathrm{~Hz}, 1 \mathrm{H}, \mathrm{Ar} H) .{ }^{13} \mathbf{C}$ NMR $\left(101 \mathrm{MHz}, \mathrm{CDCl}_{3}\right) \delta 166.6,141.5,137.1,134.1$, 132.7, 131.0, 130.4, 125.2, 117.8, 104.2 (one aromatic Carbon signal not resolved). IR v $3080(\mathrm{w})$, 2958 (w), 2925 (m), 2853 (w), 1716 (w), 1622 (s), 1603 (s), 1558 (m), 1438 (w), 1354 (w), 1341 (w), $1299(\mathrm{w}), 1223(\mathrm{w}), 1006(\mathrm{w}), 951$ (w), $834(\mathrm{w}), 830$ (w). HRMS (ESI) calcd for $\mathrm{C}_{11} \mathrm{H}_{8} \mathrm{IO}_{2} \mathrm{~S}^{+}[\mathrm{M}+\mathrm{H}]^{+}$330.9284; found 330.9291 .

\section{1-(3-1H-2,5-dimethylfuran)-1 $H$-1 $1 \lambda_{3}$-benzo $[b]$ iodo-3(2H)-one (9t)}<smiles>Cc1cc(I2COC(=O)c3ccccc32)c(C)o1</smiles>

$9 \mathbf{t}$

Starting from commercially available 2,5-dimethylfuran $(107 \mu \mathrm{L}, 1.00$ mmol) and using $\operatorname{In}(\mathrm{OTf})_{3}$ as the Lewis Acid (20 mol\%), after 24 hours 1-

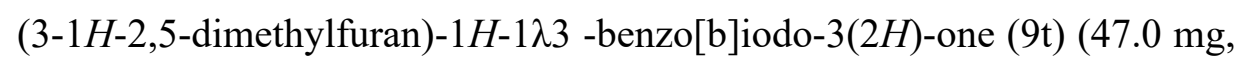
$0.137 \mathrm{mmol}, 14 \%$ yield) was obtained as a brown resin. Rf: 0.3 (DCM:MeOH 9:1). ${ }^{1} \mathbf{H}$ NMR (400 MHz, $\left.\mathrm{CDCl}_{3}\right) \delta 8.28(\mathrm{dd}, J=7.4,1.8 \mathrm{~Hz}$, 1H, ArH), 7.50 (t, $J=7.3 \mathrm{~Hz}, 1 \mathrm{H}, \operatorname{Ar} H), 7.43$ (td, $J=7.7,7.3,1.8 \mathrm{~Hz}, 1 \mathrm{H}, \operatorname{Ar} H), 7.00$ (d, $J=8.1$ $\mathrm{Hz}, 1 \mathrm{H}, \mathrm{ArH}), 6.21$ (s, 1H, ArH), 2.42 (s, 3H, FuraneCH $\left.\mathrm{CH}_{3}\right), 2.31$ (s, 3H, FuraneCH $\left.{ }_{3}\right) .{ }^{13} \mathbf{C}$ NMR $\left(101 \mathrm{MHz}, \mathrm{CDCl}_{3}\right) \delta 166.9,158.9,154.2,133.5,133.1,132.4,130.4,125.4,115.0,110.4,88.0$, 13.4, 13.2. IR $v 3430(\mathrm{w}), 3111(\mathrm{w}), 3065(\mathrm{w}), 2923(\mathrm{w}), 2854(\mathrm{w}), 1607$ (s), $1559(\mathrm{~m}), 1438(\mathrm{~m})$, 1348 (m), 1298 (w), 1229 (w), 1128 (w), 1039 (w), 1007 (w), 926 (w), 831 (m). HRMS (ESI) calcd for $\mathrm{C}_{13} \mathrm{H}_{12} \mathrm{IO}_{3}{ }^{+}[\mathrm{M}+\mathrm{H}]^{+}$342.9826; found 342.9827. 


\section{$\underline{\text { 2.3 Preparation of } \beta \text {-Phenyliodonioindole Tetrafluoroborate (18). }}$}<smiles>c1ccc2[nH]ccc2c1</smiles>

1) 5.0 equiv. $\mathrm{KOH}$

$\underset{\text { 2) }}{\stackrel{\mathrm{MeOH} 1 \mathrm{M}, 0 \text { equiv. PIDA }}{ } 0^{\circ} \mathrm{C}}$ $0{ }^{\circ} \mathrm{C}$

34

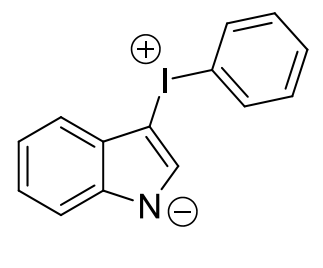

Betaine I
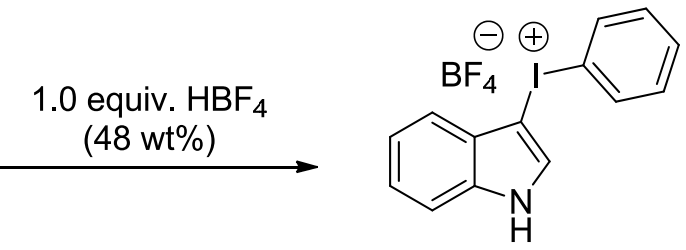

18

Following a reported procedure, ${ }^{13}$ to a stirred solution of fine crushed $\mathrm{KOH}(5.61 \mathrm{~g}, 100 \mathrm{mmol}$, 5.00 equiv.) in $\mathrm{MeOH}(20.0 \mathrm{~mL}, 1.0 \mathrm{M}), 1 H$-indole 34 (2.34 g, $20.0 \mathrm{mmol}, 1.00$ equiv.) was added in portions at $0{ }^{\circ} \mathrm{C}$. The resulting mixture was left stirring at $0{ }^{\circ} \mathrm{C}$ until complete dissolution of the $1 H$-indole $(1.5 \mathrm{~h})$. Then PIDA (6.44 g, $20.0 \mathrm{mmol}, 1.00$ equiv.) was added portionwise and the reaction mixture left stirring at $0{ }^{\circ} \mathrm{C}$ for $1.5 \mathrm{~h}$. Betaine $\mathbf{I}$ intermediate (dark yellow green solid in suspension) was then removed by filtration over a glass-synthered funnel, washed with cold $\mathrm{MeOH}$ and $\mathrm{CHCl}_{3}$ and air dried in the dark at $0{ }^{\circ} \mathrm{C}$. Betaine $\mathbf{I}$ intermediate $(2.20 \mathrm{~g}, 6.89 \mathrm{mmol}, 35 \%$ yield $)$ was obtained as a dark yellow green amorphous solid.

Caution: Betaine I is a highly unstable intermediate, it is reported to detonate at room temperature; do NOT grind it, it may explode. The Betaine I intermediate decomposes at $-20^{\circ} \mathrm{C}$, it is recommended to be used immediately after its preparation.

$\mathrm{HBF}_{4}\left(450 \mu \mathrm{L}, 6.89 \mathrm{mmol}, 1.00\right.$ equiv., 48 wt.\% solution in $\left.\mathrm{H}_{2} \mathrm{O}\right)$ was added to EtOH $(10 \mathrm{~mL})$ and the resulting solution cooled at $-15^{\circ} \mathrm{C}$; then Betaine I intermediate $(2.20 \mathrm{~g}, 6.89 \mathrm{mmol}, 1.00$ equiv.) was added portionwise under vigorous stirring at $-15^{\circ} \mathrm{C}$. After all of Betaine $\mathbf{I}$ had been added, the dark brown resulting reaction mixture was diluted with $\mathrm{Et}_{2} \mathrm{O}(50 \mathrm{~mL})$ and stirred for $1.5 \mathrm{~h}$ at -15 ${ }^{\circ} \mathrm{C}$. Then stirring was stopped and the reaction left at $-15{ }^{\circ} \mathrm{C}$ for $30 \mathrm{~min}$. The resulting precipitate was then removed by filtration over a glass-synthered funnel and dried at $0{ }^{\circ} \mathrm{C}$ to give $\beta$ Phenyliodonioindole Tetrafluoroborate 18 (2.10 g, $5.16 \mathrm{mmol}, 75 \%$ yield $)$ as a yellowish green amorphous solid.

[13] B. Y. Karele, L. é. Treigute, S. V. Kalnin', I. P. Grinberga, O. Y. Neiland, Chem. Heterocycl. Compd. 1974, 10, 189-192. 
Caution: $\beta$-Phenyliodonioindole Tetrafluoroborate 18 is an unstable salt and it was immediately used after its preparation.

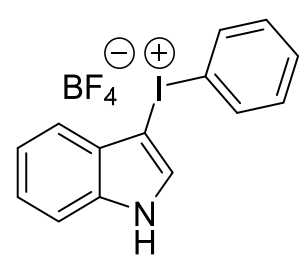

18

${ }^{1}$ H NMR (400 MHz, DMSO-d $) \delta 12.32($ brs, $1 \mathrm{H}, \mathrm{N} H), 8.40(\mathrm{~d}, J=2.7 \mathrm{~Hz}$, $1 \mathrm{H}, \mathrm{ArH}), 8.14-8.12(\mathrm{~m}, 2 \mathrm{H}, \mathrm{ArH}), 7.74(\mathrm{~m}, 1 \mathrm{H}, \mathrm{ArH}), 7.58-7.55(\mathrm{~m}, 2 \mathrm{H})$, $7.47-7.43(\mathrm{~m}, 2 \mathrm{H}), 7.35-7.16(\mathrm{~m}, 2 \mathrm{H})$. (presence of $\mathrm{Et}_{2} \mathrm{O}$ residual solvent as the NMR analysis was performed on the slightly wet compound to prevent decomposition). ${ }^{13} \mathbf{C}$ NMR (101 MHz, DMSO-d 6 ) $\delta 135.8,135.1,134.0,131.5$, 127.1, 123.6, 121.9, 118.8, 117.3, 113.0, 78.0. (one aromatic Carbon signal not resolved). IR v 3375 (s), 3132 (w), 1561 (m), 1491 (m), 1472 (s), 1411 (s), 1340 (m), 1328 (m), 1279 (s), 1244 (s). HRMS (ESI) calcd for $\mathrm{C}_{14} \mathrm{H}_{11} \mathrm{IN}\left[\mathrm{M}^{+}\right]$319.9931; found 319.9932 . 


\subsection{Synthesis of Starting Materials.}

All commercially available chemicals were purchased from the suppliers quoted in Paragraph 1.0 of Supplementary Informations: these chemicals were purified through a short plug of celite prior to their use in catalysis. The synthesis of non commercial available compounds is presented below.

\section{Methyl 4-(pyridin-2-yl)benzoate (44)}

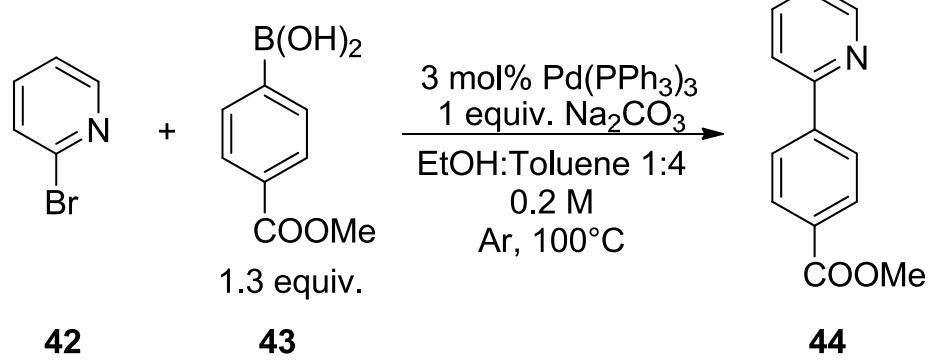

Following a reported procedure, ${ }^{14}$ to a solution of commercially available 2-bromopyridine $\mathbf{4 2}(100$ $\mu \mathrm{L}, 1.00 \mathrm{mmol})$ in a mixture of $4 / 1$ toluene/EtOH $(5 \mathrm{~mL}, 0.2 \mathrm{M})$ was added $\mathrm{Na}_{2} \mathrm{CO}_{3}(106 \mathrm{mg}, 1.00$ mmol, 1.00 equiv.) followed by $\mathrm{Pd}\left(\mathrm{PPh}_{3}\right)_{4} \quad(34.7 \mathrm{mg}, 30.0 \mu \mathrm{mol}, 3 \mathrm{~mol} \%)$ and (4(methoxycarbonyl)phenyl)boronic acid 43 (234 mg, $1.30 \mathrm{mmol}, 1.30$ equiv.) under argon atmosphere in a $50 \mathrm{~mL}$ two-necked flask. The reaction mixture was refluxed for $12 \mathrm{~h}$, and then cooled to room temperature. To the reaction mixture was added sat. aqueous $\mathrm{NH}_{4} \mathrm{Cl}(15 \mathrm{~mL})$, then the mixture was extracted by EtOAc $(3 \times 5 \mathrm{ml})$. The combined organic extracts were dried over $\mathrm{MgSO}_{4}$, filtered and and concentrated in vacuo. The resulting crude product was purified by flash chromatography (Pentane:EtOAc 4:1) to afford methyl 4-(pyridin-2-yl)benzoate 44 (181 mg, 0.849 mmol, $85 \%$ yield) as a white solid. ${ }^{1} \mathbf{H}$ NMR $\left(400 \mathrm{MHz}, \mathrm{CDCl}_{3}\right) \delta 8.64(\mathrm{~d}, J=4.7 \mathrm{~Hz}, 1 \mathrm{H}, \mathrm{Ar} H)$, $8.06(\mathrm{~d}, J=8.4 \mathrm{~Hz}, 2 \mathrm{H}, \operatorname{Ar} H), 7.98(\mathrm{~d}, J=8.3 \mathrm{~Hz}, 2 \mathrm{H}, \operatorname{Ar} H), 7.73-7.63$ (m, 2H, ArH), 7.19 (q, $J$ $=4.6 \mathrm{~Hz}, 1 \mathrm{H}, \mathrm{Ar} H), 3.85$ (s, 3H COOMe). ${ }^{13} \mathbf{C ~ N M R}\left(101 \mathrm{MHz}, \mathrm{CDCl}_{3}\right) \delta 166.8,156.1,149.8$, 143.4, 136.8, 130.3, 130.0, 126.7, 122.8, 120.9, 52.1.IR $v 2944$ (w), 2848 (w), 1708 (s), $1606(w)$, 1586 (m), 1466 (w), 1435 (m), 1405 (w), 1319 (w), 1274 (s), 1194 (m), 1183 (m), 1153 (w), 111 (s), $1014(\mathrm{~m}), 965(\mathrm{~m}), 868(\mathrm{w}), 830(\mathrm{w}), 797(\mathrm{w}), 754(\mathrm{vs}), 699(\mathrm{~m})$. NMR values are in accordance with the data reported in literature. ${ }^{15}$

[14] H. Mizuno, J. Takaya, N. Iwasawa, J. Am. Chem. Soc. 2011, 133, 1251-1253

[15] K. Muto, T. Hatakeyama, K. Itami, J. Yamaguchi, Org. Lett. 2016, 18, 5106-5109. 


\section{1-(Pyrimidin-2-yl)-1H-indole (46)}

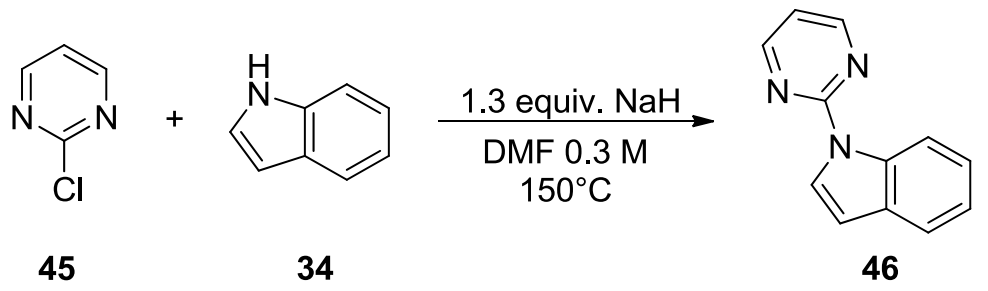

Following a reported procedure, ${ }^{16}$ commercially available $1 H$-indole $34(586 \mathrm{mg}, 5.00 \mathrm{mmol})$ was dissolved in $\mathrm{N}, \mathrm{N}$-dimethylformamide $(15.0 \mathrm{~mL}, 0.3 \mathrm{M})$ and sodium hydride $(60 \%$ suspension in mineral oil, $300 \mathrm{mg}, 7.50 \mathrm{mmol}, 1.50$ equiv.) was added at r.t. and the reaction mixture was stirred for one hour. Upon seizing of gas release, commercially available 2-chloropyrimidine 45 (573 mg, $5.00 \mathrm{mmol}, 1.00$ equiv.) was added portionwise. The reaction was heated up to $150{ }^{\circ} \mathrm{C}$ and stirred overnight. After 10 hours the reaction was allowed cooling to r.t. and was then quenched with water $(20 \mathrm{~mL})$. The majority of the solvent was removed under reduced pressure, then the crude was diluted with $\mathrm{Et}_{2} \mathrm{O}(25 \mathrm{~mL})$, the organic layer washed with brine $(3 \times 10 \mathrm{~mL})$, dried over $\mathrm{MgSO}_{4}$, filtered and concentrated under reduced pressure. Flash column chromatography (Pentane:EtOAc 4:1) afforded 1-(pyrimidin-2-yl)-1 $H$-indole 46 (926 mg, $4.74 \mathrm{mmol}, 95 \%$ yield) as a light brown oil. ${ }^{1} \mathbf{H}$ NMR (400 MHz, $\left.\mathrm{CDCl}_{3}\right) \delta 8.94(\mathrm{~d}, J=8.4 \mathrm{~Hz}, 1 \mathrm{H}, \mathrm{Ar} H), 8.75(\mathrm{~d}, J=4.8 \mathrm{~Hz}, 2 \mathrm{H}, \mathrm{Ar} H)$, $8.40(\mathrm{~d}, J=3.7 \mathrm{~Hz}, 1 \mathrm{H}, \operatorname{Ar} H), 7.75(\mathrm{~d}, J=7.8 \mathrm{~Hz}, 1 \mathrm{H}, \operatorname{Ar} H), 7.51-7.43$ (m, 1H, ArH), 7.37 (t, $J$ $=7.5 \mathrm{~Hz}, 1 \mathrm{H}, \operatorname{Ar} H), 7.07(\mathrm{t}, J=4.8 \mathrm{~Hz}, 1 \mathrm{H}, \operatorname{Ar} H), 6.83(\mathrm{~d}, J=3.6 \mathrm{~Hz}, 1 \mathrm{H}, \operatorname{Ar} H) .{ }^{13} \mathbf{C}$ NMR $(101$ $\left.\mathrm{MHz}_{\mathrm{CDCl}}\right) \delta 157.7,157.4,135.1,131.1,125.6,123.4,121.9,120.6,116.2,115.8,106.6$. IR $v$ 3138 (w), 3108 (w), 1575 (s), 1525 (m), 1456 (s), 1309 (m), 1204 (s), 1080 (m), 970 (s), 776 (m), $750(\mathrm{w}), 731(\mathrm{w})$. NMR values are in accordance with the data reported in literature. ${ }^{17}$

$(2 R, 3 R, 4 R, 5 R)-2-(A c e t o x y m e t h y l)-5-(6-p h e n y l-9 H$-purin-9-yl)tetrahydrofuran-3,4-diyl diacetate (50)

[16] M. Nishino, K. Hirano, T. Satoh, M. Miura, Angew. Chem. Int. Ed. 2012, 51, 6993-6997.

[17] L. Ackermann, A. V. Lygin, Org. Lett. 2011, 13, 3332-3335. 


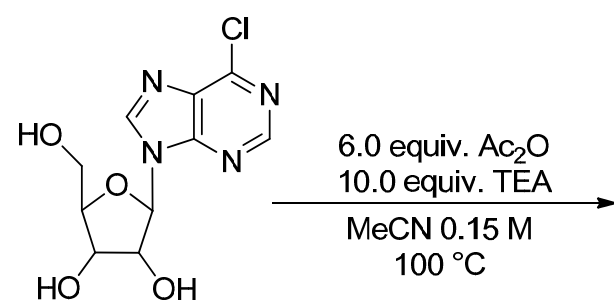

47

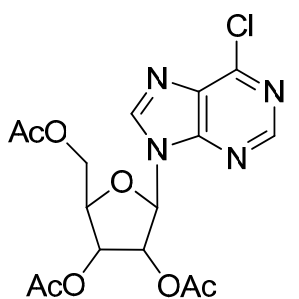

48

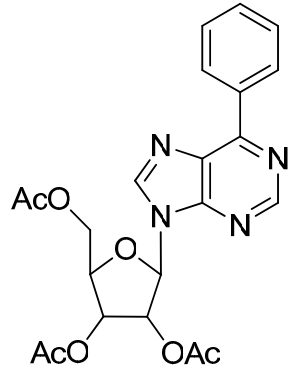

50

Following a reported procedure, ${ }^{18}$ commercially available $(2 R, 3 R, 4 S, 5 R)-2$-(6-chloro-9H-purin-9yl)-5-(hydroxymethyl)tetrahydrofuran-3,4-diol 47 (573 mg, $2.00 \mathrm{mmol}, 1.00$ equiv.) was suspended in MeCN (12.5 mL, 0.15 M). Then Triethylamine (2.90 mL, $20.0 \mathrm{mmol}, 10.0$ equiv.) and Acetic Anhydride ( $1.10 \mathrm{~mL} \mu 1,12.00 \mathrm{mmol}, 6.00$ equiv.) were added at $0^{\circ} \mathrm{C}$. After stirring for $1 \mathrm{~h}$ at room temperature, the mixture was refluxed for 5 hours. The resulting solution was evaporated to dryness and EtOAc $(30 \mathrm{~mL})$ and water $(30 \mathrm{~mL})$ were added. The organic layer was separated, dried over $\mathrm{Na}_{2} \mathrm{SO}_{4}$, filtered and concentrated under reduced pressure to give a light brown oil, which was recrystallized from EtOAc/Ether to give $(2 R, 3 R, 4 R, 5 R)$-2-(acetoxymethyl)5-(6-chloro-9H-purin-9-yl)tetrahydrofuran-3,4-diyl diacetate 48 (693 mg, $1.68 \mathrm{mmol}, 84$ \% yield)

Subsequently, to a solution of $(2 R, 3 R, 4 R, 5 R)$-2-(acetoxymethyl)-5-(6-chloro-9H-purin-9yl)tetrahydrofuran-3,4-diyl diacetate $48(500 \mathrm{mg}, 1.21 \mathrm{mmol})$ in a mixture of $4 / 1$ toluene $(12 \mathrm{~mL}$, $0.1 \mathrm{M})$ was added $\mathrm{K}_{2} \mathrm{CO}_{3}\left(218 \mathrm{mg}, 1.58 \mathrm{mmol}, 1.30\right.$ equiv.) followed by $\mathrm{Pd}\left(\mathrm{PPh}_{3}\right)_{4}(42.0 \mathrm{mg}, 30.0$ $\mu \mathrm{mol}, 3 \mathrm{~mol} \%$ ) and phenylboronic acid 49 (192 $\mathrm{mg}, 1.58 \mathrm{mmol}, 1.30$ equiv.) under argon atmosphere in a $20 \mathrm{~mL}$ two-necked flask. The reaction mixture was refluxed for $12 \mathrm{~h}$, and then cooled to room temperature. To the reaction mixture was added sat. aqueous $\mathrm{NH}_{4} \mathrm{Cl}(15 \mathrm{~mL})$, then the mixture was extracted by EtOAc $(3 \times 5 \mathrm{~mL})$. The combined organic extracts were dried over $\mathrm{MgSO}_{4}$, filtered and and concentrated in vacuo. The resulting crude product was purified by flash chromatography (Pentane:EtOAc 4:1) to afford (2R,3R,4R,5R)-2-(acetoxymethyl)-5-(6-phenyl9H-purin-9-yl)tetrahydrofuran-3,4-diyl diacetate 50 (523 $\mathrm{mg}, 1.15 \mathrm{mmol}, 95 \%$ yield) as a white solid. ${ }^{1} \mathbf{H}$ NMR $\left(400 \mathrm{MHz}, \mathrm{CDCl}_{3}\right) \delta 8.95(\mathrm{~s}, 1 \mathrm{H}, \mathrm{ArH}), 8.68$ (dd, $\left.J=8.0,1.8 \mathrm{~Hz}, \mathrm{ArH}\right), 8.21$ (s, $1 \mathrm{H}, \mathrm{ArH}), 7.52-7.43(\mathrm{~m}, 3 \mathrm{H}, \mathrm{ArH}), 6.22$ (d, $J=5.3 \mathrm{~Hz}, 1 \mathrm{H}, C H), 5.95(\mathrm{t}, J=5.4 \mathrm{~Hz}, 1 \mathrm{H}, \mathrm{CH})$,

[18] M. A. Ali, X. Yao, H. Sun, H. Lu, Org. Lett. 2015, 17, 1513-1516. 
$5.64(\mathrm{~m}, 1 \mathrm{H}, \mathrm{CH}), 4.45-4.24\left(\mathrm{~m}, 3 \mathrm{H}, \mathrm{CH}+\mathrm{CH}_{2}\right), 2.08\left(\mathrm{~s}, 3 \mathrm{H}, \mathrm{CH}_{3}\right), 2.05\left(\mathrm{~s}, 3 \mathrm{H}, \mathrm{CH}_{3}\right), 2.00$ (s, $3 \mathrm{H}, \mathrm{CH}_{3}{ }^{13} \mathrm{C}$ NMR $\left(101 \mathrm{MHz}, \mathrm{CDCl}_{3}\right) \delta 169.9,169.2,169.0,154.7,152.1,151.6,142.5,135.0$, 131.2, 130.7, 129.4, 128.2, 86.1, 79.9, 72.7, 70.2, 62.7, 20.3, 20.1, 20.0. IR v 2926 (m), 1749 (s), $1583(\mathrm{~s}), 1566$ (s), 1439 (m), 1220 (s), 1101 (m), $766(\mathrm{~m}), 693(\mathrm{~m})$. NMR values are in accordance with the data reported in literature. ${ }^{18}$ 


\subsection{Procedure for Thio-indolization via Lewis-acid activated IndoleBX}<smiles>COc1cccc(S)c1</smiles>

10

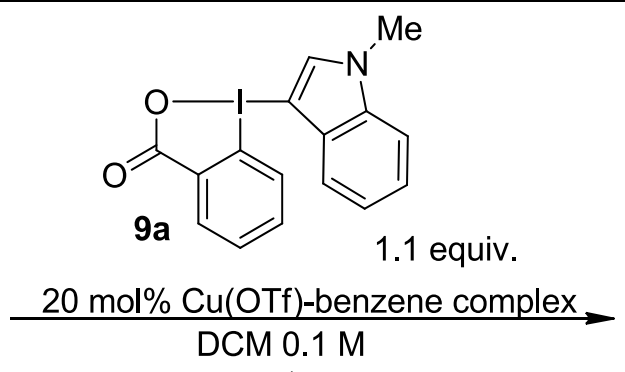

r.t<smiles>COc1cccc(Sc2cn(C)c3ccccc23)c1</smiles>

11

Commercially available 3-methoxybenzenethiol $10(25.0 \mu \mathrm{L}, 0.200 \mathrm{mmol}, 1.00$ equiv.) was dissolved in dry DCM $(2.0 \mathrm{~mL}, 0.1 \mathrm{M})$ under nitrogen atmosphere. Then IndoleBX 9a $(75.0 \mathrm{mg}$, $0.200 \mathrm{mmol}, 1.00$ equiv.) and the reaction left stirring at r.t. overnite. The majority of the solvent was removed under reduced pressure, then the crude was diluted with EtOAc $(25 \mathrm{~mL})$, the organic layer washed with brine $(3 \times 10 \mathrm{~mL})$, dried over $\mathrm{MgSO}_{4}$, filtered and concentrated under reduced pressure. Flash column chromatography (Pentane:EtOAc 4:1) afforded 3-((3methoxyphenyl)thio)-1-methyl-1H-indole $11(18.1 \mathrm{mg}, 67.0 \mu \mathrm{mol}, 34 \%$ yield) as a light yellow oil. ${ }^{1} \mathbf{H}$ NMR $\left(400 \mathrm{MHz}, \mathrm{CDCl}_{3}\right) \delta 7.62(\mathrm{~d}, J=7.9 \mathrm{~Hz}, 1 \mathrm{H}, \mathrm{Ar} H), 7.38(\mathrm{~d}, J=8.1 \mathrm{~Hz}, 1 \mathrm{H}, \mathrm{Ar} H)$, 7.34 (s, 1H, ArH), 7.30 (m, 1H, ArH), 7.16 (ddd, $J=7.9,6.8,0.9 \mathrm{~Hz}, 1 \mathrm{H}, \operatorname{Ar} H), 7.06$ (t, $J=8.0$ $\mathrm{Hz}, 1 \mathrm{H}, \operatorname{Ar} H), 6.71-6.63(\mathrm{~m}, 2 \mathrm{H}, \operatorname{Ar} H), 6.59$ (dd, $J=8.1,2.5 \mathrm{~Hz}, 1 \mathrm{H}, \operatorname{Ar} H), 3.85$ (s, 3H, NMe), $3.68(\mathrm{~s}, 3 \mathrm{H}, \mathrm{OMe}) .{ }^{13} \mathrm{C}$ NMR $\left(101 \mathrm{MHz}, \mathrm{CDCl}_{3}\right) \delta 160.0,141.4,137.7,135.3,129.7,129.6,122.7$, $120.7,119.9,118.3,111.6,110.3,109.8,100.4,55.4,33.4$. NMR values are in accordance with the data reported in literature. ${ }^{19}$

[19] H. Qi, T. Zhang, K. Wan, M. Luo, J. Org. Chem. 2016, 81, 4262-4268. 


\subsection{Optimization of the Rh-Catalyzed Indolization of Arenes via $\mathrm{C}-\mathrm{H}$ activation.}

In a vial, 2-phenylpyridine $12(14.0 \mu \mathrm{l}, 0.100 \mathrm{mmol}), 1$-(3-1-methyl-1H-indole)- $1 H-1 \lambda_{3}$ benzo[b]iodo-3(2H)-one 9a (1.10 equiv.), the catalyst system $(1.25-5 \mathrm{~mol} \%)$ and the relative additive (2.5-20 mol\%) were dissolved in the appropriate dry solvent $(0.1 \mathrm{M})$ under nitrogen. The reaction mixture was degassed (freeze-thaw-pump) and then stirred at the reported $\mathrm{T}$ in ${ }^{\circ} \mathrm{C}$ overnight. The reaction mixture was then allowed to cool to r.t., the organic layer was washed with sat. aqueous $\mathrm{NaHCO}_{3}(2 \mathrm{~mL})$, and the solvent was removed under reduced pressure. Flash column chromatography (Pentane:EtOAc 4:1) afforded the desired product 13a (see compound 13a's characterization for all the chemical data).

Table S3: Screening of solvents

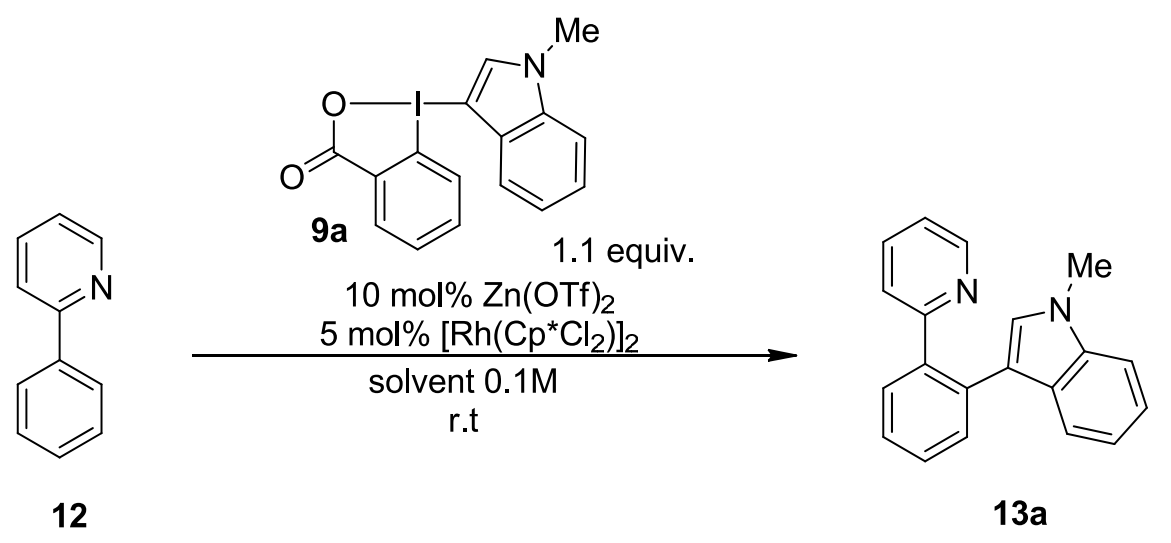

\begin{tabular}{ccc}
\hline Entry & Solvent & Yield\%a $^{\mathbf{a}}$ \\
\hline 1 & DCM & $25 \%$ \\
2 & DCE & $55 \%$ \\
3 & $\mathrm{MeOH}$ & - \\
4 & $\mathrm{EtOH}$ & - \\
5 & $\mathrm{TFE}$ & $16 \%$ \\
6 & $\mathrm{Chlorobenzene}$ & - \\
7 & $1,2-$ Chlorobenzene & - \\
8 & Toluene & - \\
9 & DMF $\left(110^{\circ} \mathrm{C}\right)$ & $66 \%$ \\
\hline
\end{tabular}

a) Substrate $12(0.100 \mathrm{mmol})$, IndoleBX 9a $(0.110 \mathrm{mmol}),\left[\mathrm{Rh}\left(\mathrm{Cp}^{*} \mathrm{Cl}_{2}\right)\right]_{2}$

(5 mol\%), $\mathrm{Zn}(\mathrm{OTf})_{2}(10 \mathrm{~mol} \%)$ and solvent $(0.1 \mathrm{M})$ at $25^{\circ} \mathrm{C}$. Isolated yield after flash chromatography is given. 
Table S4: Screening of additives

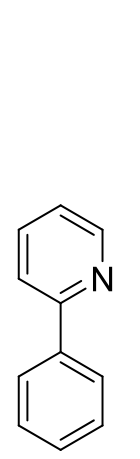

12

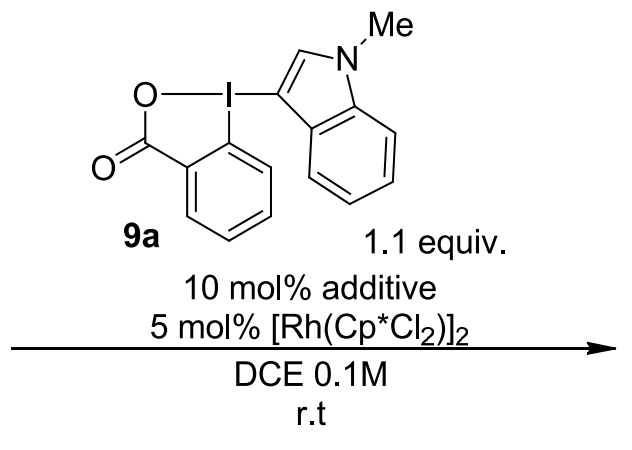

r.t

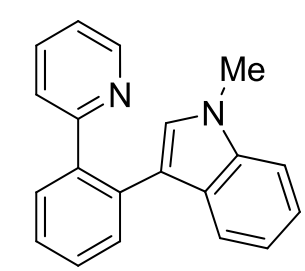

$13 a$

\begin{tabular}{|c|c|c|}
\hline Entry & Additive & Yield\% $\%^{a}$ \\
\hline 1 & - & - \\
\hline 2 & $\mathrm{Zn}(\mathrm{OTf})_{2}$ & $55 \%{ }^{\mathrm{b}}$ \\
\hline 3 & $\mathrm{Sc}(\mathrm{OTf})_{3}$ & $56 \%{ }^{\mathrm{c}}$ \\
\hline 4 & $\mathrm{~K}_{2} \mathrm{CO}_{3}$ & $28 \%$ \\
\hline 5 & $\mathrm{Zn}\left(\mathrm{NTf}_{2}\right)_{2}$ & $56 \%$ \\
\hline 6 & $\mathrm{AgNTf}_{2}$ & - \\
\hline 7 & $\mathrm{AgSbF}_{6}^{\mathrm{d}}$ & $17 \%$ \\
\hline 8 & $\mathrm{NaOAc}$ & $69 \%$ \\
\hline 9 & KOAc & $37 \%$ \\
\hline 10 & NaOPiv & $72 \%$ \\
\hline 11 & KOPiv & $49 \%$ \\
\hline 12 & CsOPiv & $36 \%$ \\
\hline
\end{tabular}

a) Substrate $12(0.100 \mathrm{mmol})$, IndoleBX 9a $(0.110 \mathrm{mmol}),\left[\mathrm{Rh}\left(\mathrm{Cp}^{*} \mathrm{Cl}_{2}\right)\right]_{2}(5$ mol\%), additive (10 mol\%) and DCE $(0.1 \mathrm{M})$ at $25{ }^{\circ} \mathrm{C}$. Isolated yield after flash chromatography is given. b) incomplete conversion, remaining starting material completely recovered. c) decomposition observed. d) $\mathrm{T}$ of the reaction is $50^{\circ} \mathrm{C}$. 
Table S5: Screening of the catalyst:additive ratio at different $\mathrm{T}^{\circ} \mathrm{C}$.<smiles>c1ccc(-c2ccccn2)cc1</smiles>

12

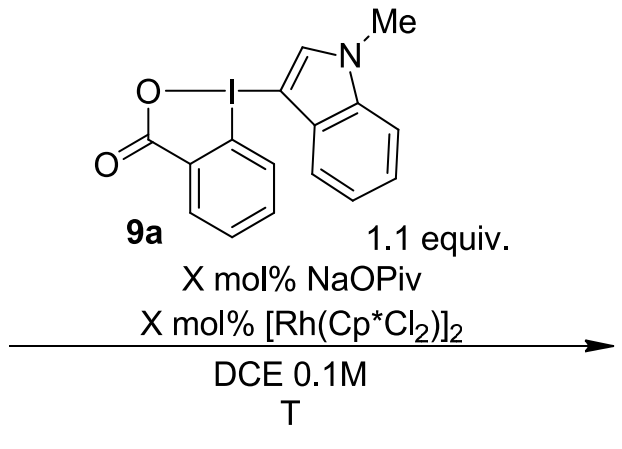

$\mathrm{T}$<smiles>Cn1cc(-c2ccccc2-c2ccccn2)c2ccccc21</smiles>

$13 a$

\begin{tabular}{|c|c|c|c|c|}
\hline Entry & Catalyst (mol\%) & Additive (mol\%) & $\mathbf{T}^{\circ} \mathbf{C}$ & Yield\%a \\
\hline 1 & $\begin{array}{c}{\left[\mathrm{Rh}\left(\mathrm{Cp}^{*} \mathrm{Cl}_{2}\right)_{2}\right]} \\
(1.25) / \mathrm{AgSbF}_{6}(2.5)\end{array}$ & NaOPiv (2.5) & r.t. & traces $^{\mathrm{b}}$ \\
\hline 2 & $\begin{array}{c}{\left[\mathrm{Rh}\left(\mathrm{Cp}^{*} \mathrm{Cl}_{2}\right)_{2}\right]} \\
(1.25) / \mathrm{AgSbF}_{6}(10)\end{array}$ & NaOPiv (10) & r.t. & traces $^{\mathrm{b}}$ \\
\hline 3 & $\begin{array}{c}{\left[\mathrm{Rh}\left(\mathrm{Cp}^{*} \mathrm{Cl}_{2}\right)_{2}\right]} \\
(1.25) / \mathrm{AgSbF}_{6}(10)\end{array}$ & NaOPiv (10) & $40^{\circ} \mathrm{C}$ & traces $^{b}$ \\
\hline 4 & $\begin{array}{c}{\left[\mathrm{Rh}\left(\mathrm{Cp}^{*} \mathrm{Cl}_{2}\right)_{2}\right]} \\
(1.25) / \mathrm{AgSbF}_{6}(10)\end{array}$ & NaOPiv (10) & $50^{\circ} \mathrm{C}$ & $37 \%{ }^{\mathrm{b}}$ \\
\hline 5 & $\begin{array}{c}{\left[\mathrm{Rh}\left(\mathrm{Cp}^{*} \mathrm{Cl}_{2}\right)_{2}\right]} \\
(2.5) / \mathrm{AgSbF}_{6}(5)\end{array}$ & NaOPiv (5) & r.t. & $\operatorname{traces}^{\mathrm{c}}$ \\
\hline 6 & $\begin{array}{c}{\left[\mathrm{Rh}\left(\mathrm{Cp}^{*} \mathrm{Cl}_{2}\right)_{2}\right]} \\
(2.5) / \mathrm{AgSbF}_{6}(10)\end{array}$ & NaOPiv (10) & r.t. & $78 \%$ \\
\hline 7 & $\begin{array}{c}{\left[\mathrm{Rh}\left(\mathrm{Cp}^{*} \mathrm{Cl}_{2}\right)_{2}\right]} \\
(2.5) / \mathrm{AgSbF}_{6}(10)\end{array}$ & NaOPiv (10) & $50^{\circ} \mathrm{C}$ & $82 \%$ \\
\hline 8 & $\begin{array}{c}{\left[\mathrm{Rh}\left(\mathrm{Cp}^{*} \mathrm{Cl}_{2}\right)_{2}\right]} \\
(2.5) / \operatorname{AgSbF}_{6}(10)\end{array}$ & NaOPiv (10) & $80^{\circ} \mathrm{C}$ & $85 \%$ \\
\hline 9 & $\begin{array}{c}{\left[\mathrm{Rh}\left(\mathrm{Cp}^{*} \mathrm{Cl}_{2}\right)_{2}\right](5) / \mathrm{AgSbF}_{6}} \\
(10)\end{array}$ & NaOPiv (10) & r.t. & $90 \%$ \\
\hline
\end{tabular}

a)Substrate $12(0.100 \mathrm{mmol})$, IndoleBX 9a $(0.110 \mathrm{mmol}),\left[\mathrm{Rh}\left(\mathrm{Cp}^{*} \mathrm{Cl}_{2}\right)\right]_{2}(\mathbf{X ~ m o l} \%), \mathrm{NaOPiv}(\mathbf{X ~ m o l} \%)$ and DCE $(0.1 \mathrm{M})$ at $25^{\circ} \mathrm{C}$. Isolated yield after flash chromatography is given. b) the reaction time was 48 hours, hypervalent iodine decomposition was observed. 


\subsection{Control experiments for the Indolization of Arenes via C-H activation.}

In order to assess the superiority of our reagents in catalysis compared to other indole-transfer reagents already known in cross-coupling reactions, we tested the latter under different sets of conditions. Substrates 8a, 16 and $\mathbf{1 7}$ are commercially available, while compound $\mathbf{1 8}$ needed to be prepared and immediately used in the catalytic process.<smiles>Cn1ccc2ccccc21</smiles>

$8 a$<smiles>Cn1cc(Br)c2ccccc21</smiles>

16<smiles>Cn1cc(I)c2ccccc21</smiles>

17<smiles></smiles>

18

Substrates 8a, 16 and 17 were tested under our optimized conditions (table S6, entries 1-3) at different temperatures (r.t.-50-60-110 ${ }^{\circ} \mathrm{C}$ ): no conversion was observed. The hypervalent indolinium salt 18 was also tested in presence of $\mathrm{NaSbF}_{6}$ (table $S 6$, entry 4) as an alternative salt to promote the exchange of $\mathrm{BF}_{4}^{-}$counteranion with $\mathrm{SbF}_{6}^{-}$, but no conversion was observed. Substrates 16 and 17 were then tested under established conditions for Pd-catalyzed cross couplings of C3halogenated indoles. 3- Bromo-N-methylindole 16 was tested under Hartwig Pd-catalyzed amination conditions ${ }^{20}$ (table S6, entry 5), carbonylative Sonogashira conditions ${ }^{21}$ (table S6, entry 6) and Heck conditions ${ }^{22}$ (table S6, entry 7): the desired product was never observed. Furthermore, when 3-Iodo- $N$-methylindole 17 was tested under Suzuki and Sonogashira cross coupling conditions $^{23}$ (table S6, entries 8-9) no product was detected.

In Table $S 7$ oxidative methods were examined (table S7, entries 1,4$),{ }^{24}$ Shi conditions (table $S 7$, entries 2,5), ${ }^{25}$ and Fagnou conditions (table S7, entries 3,6), ${ }^{26}$ the desired compound was never observed.

[20] M. W. Hooper, M. Utsunomiya, J. F. Hartwig, J. Org. Chem. 2003, 68, 2861-2873.

[21] K. T. Neumann, S. R. Laursen, A. T. Lindhardt, B. Bang-Andersen, T. Skrydstrup, Org. Lett. 2014, 16, 22162219.

[22] D. S. G. Spinella, A. Izzo, Synlett 2006, 1319-1322.

[23] B. Witulski, J. R. Azcon, C. Alayrac, A. Arnautu, V. Collot, S. Rault, Synlett 2005, 771-780.

[24] J. Wencel-Delord, C. Nimphius, H. Wang, F. Glorius, Angew. Chem. Int. Ed. 2012, 51, 13001-13005.

[25] B.-J. Li, S.-L. Tian, Z. Fang, Z.-J. Shi, Angew. Chem. Int. Ed. 2008, 47, 1115-1118.

[26] D. R. Stuart, E. Villemure, K. Fagnou, J. Am. Chem. Soc. 2007, 129, 12072-12073. 
Table S6: Control experiments (I)

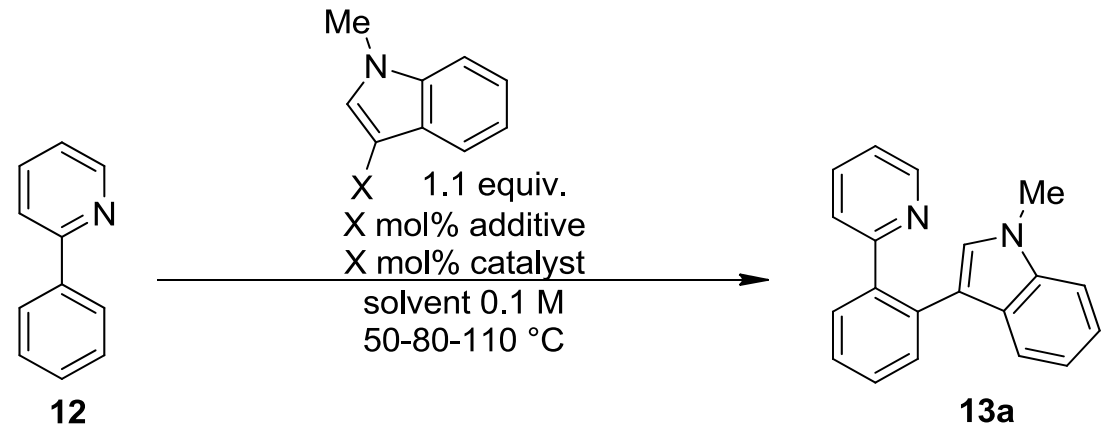

\begin{tabular}{|c|c|c|c|c|c|}
\hline Entry & $\mathbf{X}$ & Catalyst (mol\%) & Additive (mol\%) & Solvent (M) & Yield $\%^{a}$ \\
\hline 1 & H (8a) & $\begin{array}{c}{\left[\mathrm{Rh}\left(\mathrm{Cp}^{*} \mathrm{Cl}_{2}\right)_{2}\right]} \\
(2.5) / \mathrm{AgSbF}_{6}(10)\end{array}$ & NaOPiv (10) & $\operatorname{DCE}(0.1 \mathrm{M})$ & - \\
\hline 2 & I (17) & $\begin{array}{c}{\left[\mathrm{Rh}\left(\mathrm{Cp}^{*} \mathrm{Cl}_{2}\right)_{2}\right]} \\
(2.5) / \mathrm{AgSbF}_{6}(10)\end{array}$ & NaOPiv (10) & $\operatorname{DCE}(0.1 \mathrm{M})$ & - \\
\hline 3 & $\mathrm{I}^{(\mathrm{III})} \mathbf{( 1 8 )}$ & $\begin{array}{c}{\left[\mathrm{Rh}\left(\mathrm{Cp}^{*} \mathrm{Cl}_{2}\right)_{2}\right]} \\
(2.5) / \mathrm{NaSbF}_{6}(10)\end{array}$ & NaOPiv (10) & $\operatorname{DCE}(0.1 \mathrm{M})$ & - \\
\hline 4 & $\mathrm{I}^{(\mathrm{III})}(\mathbf{1 8})$ & $\begin{array}{c}{\left[\mathrm{Rh}\left(\mathrm{Cp}^{*} \mathrm{Cl}_{2}\right)_{2}\right]} \\
(2.5) / \mathrm{AgSbF}_{6}(10)\end{array}$ & NaOPiv (10) & $\operatorname{DCE}(0.1 \mathrm{M})$ & - \\
\hline 5 & $\operatorname{Br}(\mathbf{1 6})$ & $\begin{array}{l}\mathrm{Pd}(\mathrm{dba})_{2}(5) / \\
\mathrm{PPh}_{3}(5)\end{array}$ & - & Toluene $(0.2 \mathrm{M})$ & - \\
\hline 6 & $\operatorname{Br}(\mathbf{1 6})$ & $\begin{array}{c}\mathrm{PdCl}_{2}(5) / \\
\text { XanthPhos (5) }\end{array}$ & TEA (3 equiv.) & Dioxane $(0.2 \mathrm{M})$ & - \\
\hline 7 & $\operatorname{Br}(\mathbf{1 6})$ & $\begin{array}{l}\mathrm{Pd}(\mathrm{OAc})_{2}(20) / \\
\mathrm{P}(o-\mathrm{Tol})_{3}(20)\end{array}$ & TEA (3 equiv.) & Toluene $(0.2 \mathrm{M})$ & - \\
\hline 8 & I (17) & $\mathrm{PdCl}_{2} \mathrm{PPh}_{3}(5)$ & $\begin{array}{c}\mathrm{CuI}(10) / \mathrm{TEA}(3 \\
\text { equiv. })\end{array}$ & $\operatorname{DMF}(0.1 \mathrm{M})$ & - \\
\hline 9 & I (17) & $\mathrm{Pd}\left(\mathrm{PPh}_{3}\right)_{3}(2.5)$ & $\mathrm{Na}_{2} \mathrm{CO}_{3}$ (1 equiv.) & $\operatorname{DMF}(0.1 \mathrm{M})$ & - \\
\hline
\end{tabular}

a)Substrate $(0.100 \mathrm{mmol})$, Indole source $(0.110 \mathrm{mmol})$, Catalyst $(\mathbf{X} \mathrm{mol} \%)$, Additive $(\mathbf{X} \mathrm{mol} \%)$ and Solvent $(0.1 \mathrm{M})$ at $50^{\circ}-80^{\circ}-110^{\circ} \mathrm{C} . \mathrm{T}$ 
Table S7: Control experiments (II)

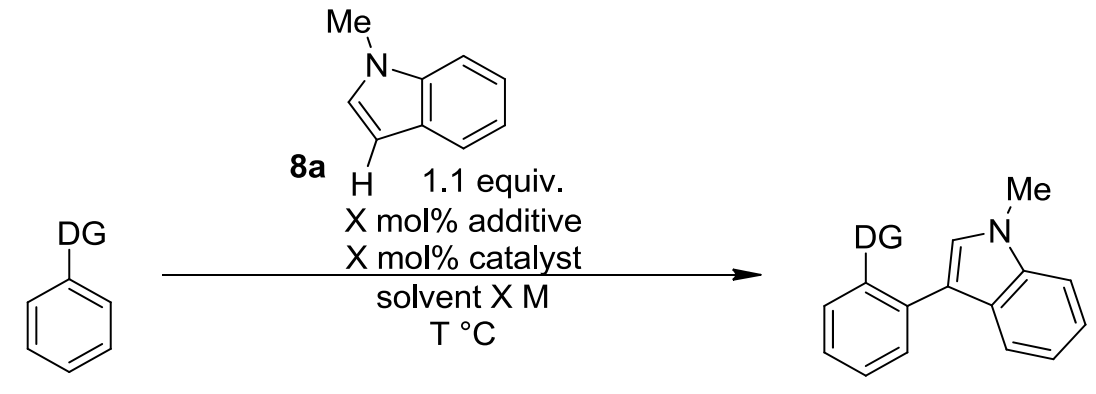

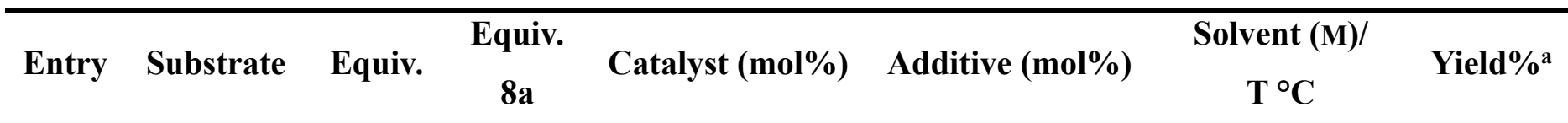

\begin{tabular}{|c|c|c|c|c|c|c|}
\hline 1 & 1 & 20 & $\begin{array}{c}{\left[\mathrm{Rh}\left(\mathrm{Cp}^{*} \mathrm{Cl}_{2}\right)_{2}\right]} \\
(2.5) / \mathrm{CsOPiv}(20)\end{array}$ & $\begin{array}{c}\mathrm{PivOH}(50) \\
2.0 \text { equiv. } \\
\mathrm{C}_{6} \mathrm{Br}_{6} / 2.2 \text { equiv. } \\
\mathrm{Cu}(\mathrm{OAc})_{2}\end{array}$ & $\begin{array}{l}\text { 2Cl-p-xylene } \\
(0.33 \mathrm{M}) / 140{ }^{\circ} \mathrm{C}\end{array}$ & - \\
\hline 2 & 1 & 6.0 & $\mathrm{Pd}(\mathrm{OAc})_{2}(10)$ & $\begin{array}{l}\mathrm{Cu}(\mathrm{OTf})_{2}(20) \\
\mathrm{O}_{2} 1 \mathrm{~atm}\end{array}$ & $\begin{array}{c}\mathrm{EtCOOH} \\
(0.2 \mathrm{M}) / 120^{\circ} \mathrm{C}\end{array}$ & - \\
\hline 3 & 60 & 1 & $\operatorname{Pd}(\mathrm{TFA})_{2}(5)$ & $\begin{array}{c}3.0 \text { equiv. } \\
\mathrm{Cu}(\mathrm{OAc})_{2} / 6.0 \\
\text { equiv. } \mathrm{PivOH}\end{array}$ & $\begin{array}{l}\text { neat in arene } \\
(0.15 \mathrm{M})\end{array}$ & - \\
\hline 4 & 1 & 20 & $\begin{array}{c}{\left[\mathrm{Rh}\left(\mathrm{Cp}^{*} \mathrm{Cl}_{2}\right)_{2}\right]} \\
(2.5) / \mathrm{CsOPiv}(20)\end{array}$ & $\begin{array}{c}\mathrm{PivOH}(50) \\
2.0 \text { equiv. } \\
\mathrm{C}_{6} \mathrm{Br}_{6} / 2.2 \text { equiv. } \\
\mathrm{Cu}(\mathrm{OAc})_{2}\end{array}$ & $\begin{array}{l}\text { 2Cl-p-xylene } \\
(0.33 \mathrm{M}) / 140{ }^{\circ} \mathrm{C}\end{array}$ & - \\
\hline 5 & 1 & 6.0 & $\mathrm{Pd}(\mathrm{OAc})_{2}(10)$ & $\begin{array}{c}\mathrm{Cu}(\mathrm{OTf})_{2}(20) \\
\mathrm{O}_{2} 1 \mathrm{~atm} .\end{array}$ & $\begin{array}{c}\mathrm{EtCOOH} \\
(0.2 \mathrm{M}) / 120^{\circ} \mathrm{C}\end{array}$ & - \\
\hline 6 & 60 & 1 & $\operatorname{Pd}(\mathrm{TFA})_{2}(5)$ & $\begin{array}{c}3.0 \text { equiv. } \\
\mathrm{Cu}(\mathrm{OAc})_{2} / 6.0 \\
\text { equiv. } \mathrm{PivOH}\end{array}$ & $\begin{array}{c}\text { neat in arene } \\
(0.15 \mathrm{M}) / 110^{\circ} \mathrm{C}\end{array}$ & - \\
\hline
\end{tabular}

a)Reaction performed on $0.1 \mathrm{mmol}$ of the limiting reagent; substrate $(\mathbf{X ~ m m o l})$, Indole $\mathbf{8 a}(\mathbf{X} \mathrm{mmol})$, Catalyst $(\mathbf{X}$ mol\%), Additive ( $\mathbf{X}$ mol\%) and Solvent $(\mathbf{X ~ M})$ at $\mathrm{T}^{\circ} \mathrm{C}$. 
Finally, in Table $S 8$ are reported control experiments employing conditions suitable for hypervalent iodine reagents such as Kita conditions (table S8, entry 1), ${ }^{27}$ Sanford conditions (table S8, entry 2), ${ }^{28}$ and Daugulis conditions (table $S 8$, entry 3 ), ${ }^{29}$ the desired compounds was not observed also in this case; All conditions screened in Tables $S 6, S 7$ and $S 8$ failed to afford the desired product, thus demonstrating the unique reactivity of our reagents.

Table S8: Control experiments (III)

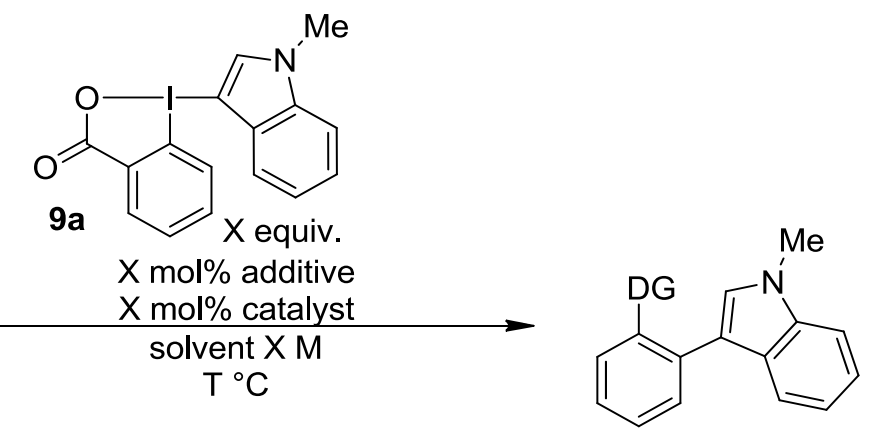

\begin{tabular}{|c|c|c|c|c|c|c|c|}
\hline Entry & Substrate & Equiv. & $\begin{array}{c}\text { Equiv. } \\
13 a\end{array}$ & Catalyst (mol\%) & $\begin{array}{l}\text { Additive } \\
\text { (mol\%) }\end{array}$ & $\begin{array}{c}\text { Solvent (M)/ } \\
\mathbf{T}^{\circ} \mathbf{C}\end{array}$ & Yield $\%{ }^{\mathrm{a}}$ \\
\hline 1 & & 1.5 & 1 & - & $\begin{array}{l}2.0 \text { equiv. } \\
\text { TMSBr }\end{array}$ & HFIP (0.1 M) & - \\
\hline 2 & 12 & 1 & 1.5 & $\mathrm{Pd}(\mathrm{OAc})_{2}(5)$ & - & $\begin{array}{c}\mathrm{MeCOOH} \\
(0.1 \mathrm{M}) / 110^{\circ} \mathrm{C}\end{array}$ & - \\
\hline 3 & & 1 & 5 & $\mathrm{Pd}(\mathrm{OAc})_{2}(5)$ & - & $\begin{array}{c}\mathrm{MeCOOH} \\
(1.5 \mathrm{M}) / 90^{\circ} \mathrm{C}\end{array}$ & - \\
\hline & 13 & & & & & & \\
\hline
\end{tabular}

a)Reaction performed on $0.1 \mathrm{mmol}$ of the limiting reagent; substrate (X mmol), Indole source 19a (X mmol), Catalyst (X mol\%), Additive (X mol\%) and Solvent (X M) at T ${ }^{\circ} \mathrm{C}$.

[27] Y. Kita, K. Morimoto, M. Ito, C. Ogawa, A. Goto, T. Dohi, J. Am. Chem. Soc. 2009, 131, 1668-1669.

[28] D. Kalyani, N. R. Deprez, L. V. Desai, M. S. Sanford, J. Am. Chem. Soc. 2005, 127, 7330-7331.

[29] O. Daugulis, V. G. Zaitsev, Angew. Chem. Int. Ed. 2005, 44, 4046-4048. 


\subsection{Scope of the Rh-Catalyzed Indolization via $\mathrm{C}-\mathrm{H}$ activation.}

\section{General Procedures GP3-GP4 for Rh-Catalyzed Hetero-arylation via C-H activation}

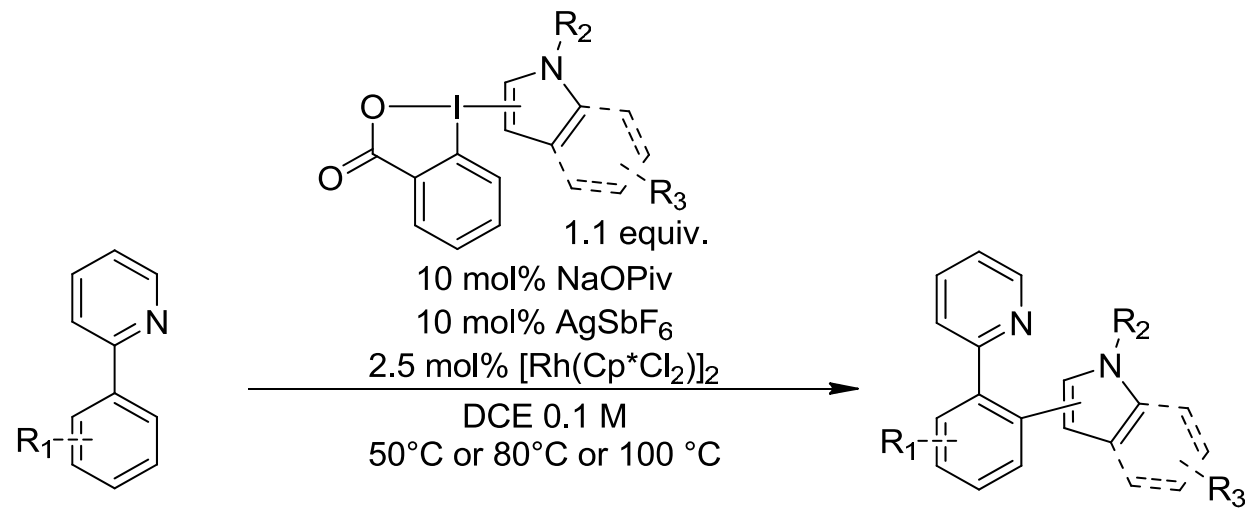

GP3: In a vial, the corresponding aryl-pyridine ( $0.300 \mathrm{mmol}, 1.00$ equiv. $)$, the relative heterocyclic hypervalent iodine reagent 9a-9r $\left(0.330 \mathrm{mmol}, 1.10\right.$ equiv.), $\left[\mathrm{Rh}\left(\mathrm{Cp}^{*} \mathrm{Cl}_{2}\right)\right]_{2}(4.60 \mathrm{mg}, 7.50 \mu \mathrm{mol}$, $2.5 \mathrm{~mol} \%$ ), $\mathrm{AgSbF}_{6}(10.3 \mathrm{mg}, 30.0 \mu \mathrm{mol}, 10 \mathrm{~mol} \%$ ) and NaOPiv (3.70 mg, $30.0 \mu \mathrm{mol}, 10 \mathrm{~mol} \%)$ were dissolved in dry 1,2-DCE $(3 \mathrm{ml}, 0.1 \mathrm{M})$ under nitrogen. the reaction mixture was degassed (freeze-thaw-pump) and stirred at the reported $\mathrm{T}$ in ${ }^{\circ} \mathrm{C}$ overnight. The reaction mixture was then allowed to cool to r.t., the organic layer was washed with sat. aquoeus $\mathrm{NaHCO}_{3}(2 \mathrm{ml})$, and the solvent was removed under reduced pressure. Flash column chromatography (Pentane:EtOAc) afforded the desired products 13a-13r.

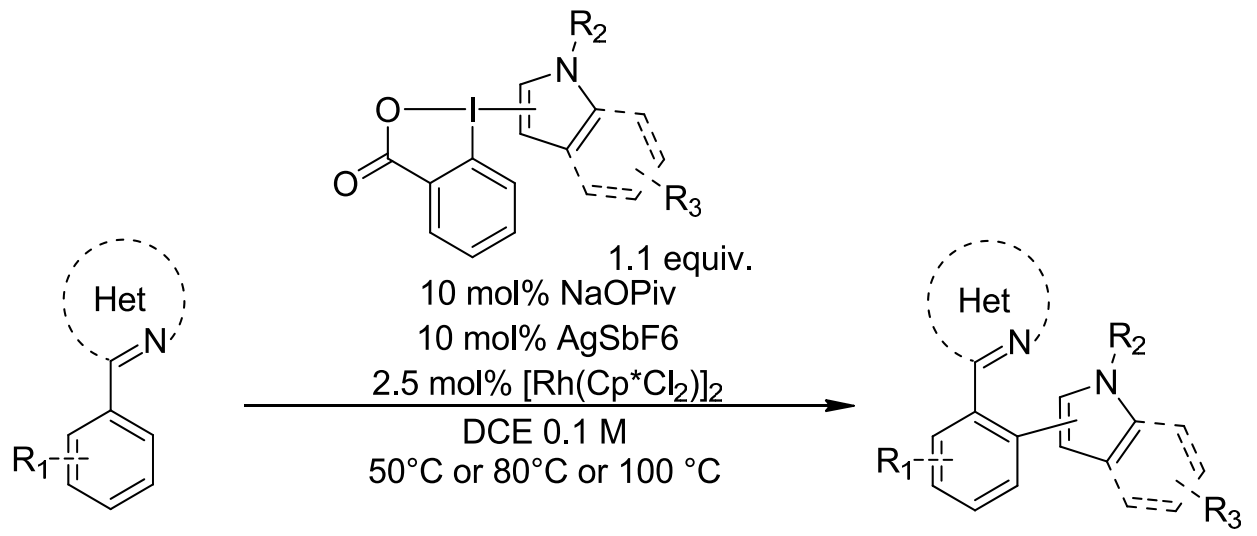

GP4: In a screw capped vial, the corresponding heterocycle $(0.300 \mathrm{mmol}, 1.00$ equiv.), the relative heterocyclic hypervalent iodine reagent 9a $(0.330 \mathrm{mmol}, 1.10$ equiv. $),\left[\mathrm{Rh}\left(\mathrm{Cp}^{*} \mathrm{Cl}_{2}\right)\right]_{2}(4.60 \mathrm{mg}$, $7.50 \mu \mathrm{mol}, 2.5 \mathrm{~mol} \%$ ), $\mathrm{AgSbF}_{6}(10.3 \mathrm{mg}, 30.0 \mu \mathrm{mol}, 10 \mathrm{~mol} \%$ ) and NaOPiv (3.70 mg, $30.0 \mu \mathrm{mol}$, $10 \mathrm{~mol} \%$ ) were dissolved in dry 1,2-DCE $(3 \mathrm{ml}, 0.1 \mathrm{M})$ under nitrogen. the reaction mixture was 
degassed (freeze-thaw-pump) and stirred at the reported $\mathrm{T}$ in ${ }^{\circ} \mathrm{C}$ overnight. The reaction mixture was then allowed to cool to r.t., the organic layer was washed with sat. aqueous $\mathrm{NaHCO}_{3}(2 \mathrm{~mL})$, and the solvent was removed under reduced pressure. Flash column chromatography (Pentane:EtOAc) afforded the desired products 19-25.<smiles>Cn1cc(-c2ccccc2-c2ccccn2)c2ccccc21</smiles>

13a $82 \%$ yield<smiles>Cn1cc(-c2ccccc2-c2ccccn2)c2cc(Cl)ccc21</smiles>

$81 \%$ yield<smiles>c1ccc(-c2ccccc2-c2c[nH]c3ccccc23)nc1</smiles>

13b

$74 \%$ yield<smiles>Cn1cc(-c2ccccc2-c2ccccn2)c2cc(I)ccc21</smiles>

$13 \mathbf{g}$

$74 \%$ yield<smiles>Cn1cccc1-c1ccccc1-c1ccccn1</smiles>

131

13k $58 \%$ yield<smiles>c1ccc(Cn2ccc(-c3ccccc3-c3ccccn3)c2)cc1</smiles>

$83 \%$ yield<smiles>c1ccc(CCCn2cc(-c3ccccc3-c3ccccn3)c3ccccc32)cc1</smiles>

$13 \mathrm{c}$

$74 \%$ yield<smiles>Cn1cc(-c2ccccc2-c2ccccn2)c2cc(Cc3ccccc3)ccc21</smiles>

$13 \mathrm{~h}$ $59 \%$ yield<smiles>c1ccc(Cn2cccc2-c2ccccc2-c2ccccn2)cc1</smiles>

$13 \mathrm{~m}$

$68 \%$ yield<smiles>COc1ccc2c(c1)c(-c1ccccc1-c1ccccn1)cn2C</smiles>

13d

$89 \%$ yield<smiles>Cn1ccc(-c2ccccc2-c2ccccn2)c1</smiles>

$13 \mathrm{i}$

$85 \%$ yield<smiles>Cn1cc(-c2ccccc2-c2ccccn2)c2cc(F)ccc21</smiles>

$73 \%$ yield<smiles>c1ccc(-c2ccccc2-c2cc[nH]c2)nc1</smiles>

13j

$79 \%$ yield<smiles>COc1ccc(-c2ccccn2)c(-c2cn(C)c3ccccc23)c1</smiles><smiles>Cn1cc(-c2cc(Cl)ccc2-c2ccccn2)c2ccccc21</smiles><smiles>Cn1cc(-c2cc(Br)ccc2-c2ccccn2)c2ccccc21</smiles><smiles>COC(=O)c1ccc(-c2ccccn2)c(-c2cn(C)c3ccccc23)c1</smiles><smiles>Cn1cc(-c2cc(C#N)ccc2-c2ccccn2)c2ccccc21</smiles>

Figure S2: Scope with Aryl-pyridines. 


\section{1-Methyl-3-(2-(pyridin-2-yl)phenyl)-1H-indole (13a)}

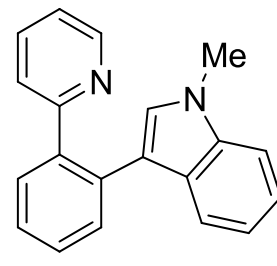

$13 a$

Starting from commercially available 2-phenylpyridine $12(43.0 \mu 1,0.300$ mmol, 1.00 equiv.) and with $1-\left(3-1\right.$-methyl- $1 \mathrm{H}$-indole)-1H-1 $\lambda_{3}$-benzo[b]iodo$3(2 \mathrm{H})$-one 9a (124 mg, $0.330 \mathrm{mmol}, 1.10$ equiv.) at $50{ }^{\circ} \mathrm{C}, 2-(2-(1-\mathrm{methyl}-1 \mathrm{H}-$ pyrrol-2-yl)phenyl)pyridine 9a (60.0 mg, $0.256 \mathrm{mmol}, 85 \%$ yield) was obtained as a pale yellow oil. Rf: 0.48 (Pentane:EtOAc 4:1). ${ }^{1} \mathbf{H}$ NMR (400 MHz, $\mathrm{CDCl}_{3}$ ) $\delta 8.65(\mathrm{~m}, 1 \mathrm{H}, \operatorname{Ar} H), 7.73(\mathrm{dd}, J=7.4,1.7 \mathrm{~Hz}, 1 \mathrm{H}, \operatorname{Ar} H), 7.59(\mathrm{~m}, 1 \mathrm{H}, \operatorname{Ar} H), 7.52-7.38$ (m, 3H, $\operatorname{Ar} H), 7.35-7.22(\mathrm{~m}, 2 \mathrm{H}, \operatorname{Ar} H), 7.19(\mathrm{ddd}, J=8.2,7.0,1.1 \mathrm{~Hz}, 1 \mathrm{H}, \operatorname{Ar} H), 7.11-6.94(\mathrm{~m}, 3 \mathrm{H}$, $\mathrm{ArH}), 6.71(\mathrm{~s}, 1 \mathrm{H}, \mathrm{NCHC}), 3.70\left(\mathrm{~s}, 3 \mathrm{H}, \mathrm{NCH}_{3}\right) .{ }^{13} \mathbf{C} \mathbf{N M R}\left(101 \mathrm{MHz}, \mathrm{CDCl}_{3}\right) \delta 159.9,149.3$, $139.8,136.8,135.1,133.3,131.0,130.5,128.4,128.2,127.0,126.7,124.9,121.6,121.2,119.9$, 119.5, 115.4, 109.1, 32.7. IR $v 3057$ (w), 2934 (w), 1725 (w), 1586 (s), 1545 (m), 1461 (s), 1425 (s), 1377 (s), 1330 (m), 1219 (m), 1162 (w), 1091 (w), 1024 (w), 942 (w), 910 (w). HRMS (ESI) calcd for $\mathrm{C}_{20} \mathrm{H}_{17} \mathrm{~N}_{2}{ }^{+}[\mathrm{M}+\mathrm{H}]^{+}$285.1386; found 285.1388.

\section{3-(2-(pyridin-2-yl)phenyl)-1H-indole (13b)}

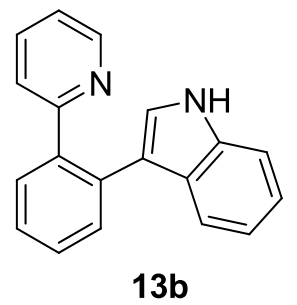

Starting from commercially available 2-phenylpyridine 8 (43.0 $\mu 1,0.300 \mathrm{mmol}$, 1.00 equiv.) and with $1-\left(3-1 H\right.$-indole)- $1 H-1 \lambda_{3}$-benzo[b]iodo-3(2H)-one 9b (120 mg, $0.330 \mathrm{mmol}, 1.10$ equiv.) at $50{ }^{\circ} \mathrm{C}$, 2-(2-(1-methyl- $1 H$-pyrrol-2yl)phenyl)pyridine 13b (60.3 $\mathrm{mg}, 0.223 \mathrm{mmol}, 74 \%$ yield) was obtained as a slightly brown foam. Rf: 0.30 (Pentane:EtOAc 4:1). ${ }^{1} \mathbf{H}$ NMR $(400 \mathrm{MHz}$, $\left.\mathrm{CDCl}_{3}\right) \delta 8.63(\mathrm{~m}, 1 \mathrm{H}, \mathrm{Ar} H), 8.40(\mathrm{~s}, 1 \mathrm{H}, \mathrm{N} H), 7.72(\mathrm{dd}, J=7.3,1.8 \mathrm{~Hz}, 1 \mathrm{H}, \operatorname{Ar} H), 7.60(\mathrm{dd}, J=$ 7.3, $1.8 \mathrm{~Hz}, 1 \mathrm{H}, \operatorname{Ar} H), 7.51-7.39$ (m, 3H, $\operatorname{Ar} H), 7.33-7.22$ (m, 2H, $\operatorname{Ar} H$ ), 7.14 (ddd, $J=8.2,6.9$, $1.2 \mathrm{~Hz}, 1 \mathrm{H}, \operatorname{Ar} H), 7.02(\mathrm{~m}, 3 \mathrm{H}, \operatorname{Ar} H), 6.76(\mathrm{~d}, J=2.5 \mathrm{~Hz}, 1 \mathrm{H}, \mathrm{NHCHC}) .{ }^{13} \mathbf{C}$ NMR $(101 \mathrm{MHz}$, $\left.\mathrm{CDCl}_{3}\right) \delta 159.8,149.0,139.8,135.9,135.3,133.3,131.0,130.5,128.5,126.9,126.6,125.0,123.7$, 122.0, 121.3, 119.9, 119.7, 116.7, 111.0. IR $v 3409$ (w), 3170 (w), 3058 (w), $2921(w), 1668(w)$, 1600 (m), 1589 (s), 1544 (w), 1489 (w), 1464 (s), 1332 (w), 1245 (m), 1153 (w), 1098 (w), 910 (m). HRMS (ESI) calcd for $\mathrm{C}_{19} \mathrm{H}_{15} \mathrm{~N}_{2}{ }^{+}[\mathrm{M}+\mathrm{H}]^{+}$271.1230; found 271.1233. 


\section{1-(3-Phenylpropyl)-3-(2-(pyridin-2-yl)phenyl)-1H-indole (13c)}

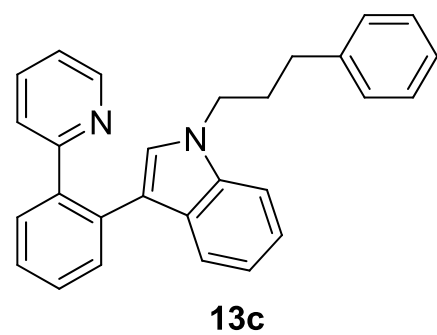

Starting from commercially available 2-phenylpyridine $12(43.0 \mu 1$, $0.300 \mathrm{mmol})$ and with 1-(3-1-(3-phenylpropyl)- $1 H$-indole)-1H-1 $\lambda_{3}-$ benzo[b]iodo-3(2H)-one $9 \mathrm{e}(159 \mathrm{mg}, 0.330 \mathrm{mmol}, 1.10$ equiv.) at 80 ${ }^{\circ} \mathrm{C}$, 1-(3-phenylpropyl)-3-(2-(pyridin-2-yl)phenyl)-1H-indole $\mathbf{1 3 c}$ (86.0 mg, $0.221 \mathrm{mmol}, 74 \%$ yield) was obtained as an orange oil. Rf: 0.48 (Pentane:EtOAc 4:1). ${ }^{1} \mathbf{H}$ NMR $\left(400 \mathrm{MHz}, \mathrm{CDCl}_{3}\right) \delta 8.67(\mathrm{~m}, 1 \mathrm{H}, \mathrm{Ar} H), 7.76(\mathrm{dd}, J=7.4$, $1.7 \mathrm{~Hz}, 1 \mathrm{H}, \operatorname{Ar} H), 7.67(\mathrm{dd}, J=7.4,1.5 \mathrm{~Hz}, 1 \mathrm{H}, \operatorname{Ar} H), 7.58(\mathrm{~d}, J=7.9 \mathrm{~Hz}, 1 \mathrm{H}, \operatorname{Ar} H), 7.51$ (td, $J=$ 7.4, 1.7 Hz, 1H, ArH), 7.47 (td, $J=7.4,1.5 \mathrm{~Hz}, 1 \mathrm{H}, \operatorname{Ar} H$ ), $7.34-7.19$ (m, 6H, $\operatorname{Ar} H$ ), 7.09 (m, 3H, $\operatorname{Ar} H), 7.06-7.01(\mathrm{~m}, 2 \mathrm{H}, \operatorname{Ar} H), 6.71\left(\mathrm{~s}, 1 \mathrm{H}, \mathrm{CH}_{2} \mathrm{NCHC}\right), 4.04(\mathrm{t}, J=6.8 \mathrm{~Hz}, 2 \mathrm{H}$, $\mathrm{PhCH}_{2} \mathrm{CH}_{2} \mathrm{CH}_{2} \mathrm{~N}$ ), $2.48\left(\mathrm{dd}, J=8.6,6.7 \mathrm{~Hz}, 2 \mathrm{H}, \mathrm{PhCH}_{2} \mathrm{CH}_{2} \mathrm{CH}_{2} \mathrm{~N}\right), 2.08$ (dq, $J=9.0,6.9 \mathrm{~Hz}, 2 \mathrm{H}$, $\left.\mathrm{PhCH}_{2} \mathrm{CH}_{2} \mathrm{CH}_{2} \mathrm{~N}\right) .{ }^{13} \mathrm{C}$ NMR $\left(101 \mathrm{MHz}, \mathrm{CDCl}_{3}\right) \delta 160.0,149.1,140.8,139.8,136.0,135.2,133.3$, $130.9,130.5,128.5,128.3,127.4,127.3,126.7,126.1,125.1,121.6,121.3,119.9,119.6,115.2$, 109.3, 45.4, 32.7, 31.3 (one Carbon signal not resolved). IR v 3059 (w), 3027 (w), 2932 (w), 1602 (w), 1585 (m), 1547 (w), 1496 (w), 1462 (s), 1424 (w), 1392 (w), 1372 (w), 1334 (w), 1167 (w), 1024 (w), 911 (w). HRMS (ESI) calcd for $\mathrm{C}_{28} \mathrm{H}_{25} \mathrm{~N}_{2}{ }^{+}[\mathrm{M}+\mathrm{H}]^{+}$389.2012; found 389.2016.

\section{5-Methoxy-1-methyl-3-(2-(pyridin-2-yl)phenyl)-1H-indole (13d)}<smiles>COc1ccc2c(c1)c(-c1ccccc1-c1ccccn1)cn2C</smiles>

$13 d$

Starting from commercially available 2-phenylpyridine $12(43.0 \mu 1,0.300$ mmol) and with 1-(3-5-methoxy-1-methyl-1H-indole)-1H-1 $\lambda_{3} \quad$ benzo[b]iodo-3(2H)-one $9 \mathrm{~g}$ (134 $\mathrm{mg}, 0.330 \mathrm{mmol}, 1.10$ equiv.) at $50{ }^{\circ} \mathrm{C}, 5$ methoxy-1-methyl-3-(2-(pyridin-2-yl)phenyl)- $1 H$-indole 13d $(84.0 \mathrm{mg}$, $0.267 \mathrm{mmol}, 89 \%$ yield) was obtained as a yellow oil. Rf: 0.40 (Pentane:EtOAc 4:1). ${ }^{1} \mathbf{H}$ NMR $\left(400 \mathrm{MHz}, \mathrm{CDCl}_{3}\right) \delta 8.67(\mathrm{dd}, \mathrm{J}=4.9,0.9 \mathrm{~Hz}, 1 \mathrm{H}, \mathrm{ArH}), 7.76(\mathrm{dd}$, $\mathrm{J}=7.2,1.9 \mathrm{~Hz}, 1 \mathrm{H}, \operatorname{Ar} H), 7.56(\mathrm{dd}, \mathrm{J}=7.2,1.9 \mathrm{~Hz}, 1 \mathrm{H}, \operatorname{Ar} H), 7.57-7.42(\mathrm{~m}, 2 \mathrm{H}, \operatorname{Ar} H), 7.26(\mathrm{td}$, $\mathrm{J}=7.7,1.9 \mathrm{~Hz}, 1 \mathrm{H}, \operatorname{Ar} H), 7.14(\mathrm{~d}, \mathrm{~J}=8.9 \mathrm{~Hz}, 1 \mathrm{H}, \operatorname{Ar} H), 7.10-7.02(\mathrm{~m}, 2 \mathrm{H}, \operatorname{Ar} H), 6.84(\mathrm{~s}, 1 \mathrm{H}$, NCHC), 6.79 (dd, J = 8.9, $2.4 \mathrm{~Hz}, 1 \mathrm{H}, \operatorname{ArH}), 6.67$ (d, J = 2.4 Hz, 1H, CCHCOMe), 3.71 (s, 3H, $\left.\mathrm{NCH}_{3}\right), 3.64\left(\mathrm{~s}, 3 \mathrm{H}, \mathrm{OCH}_{3}\right) .{ }^{13} \mathbf{C}$ NMR $\left(101 \mathrm{MHz}, \mathrm{CDCl}_{3}\right) \delta 159.9,153.9,149.3,139.4,135.2$, 133.4, 132.0, 131.0, 130.5, 128.5, 128.3, 126.9, 126.8, 124.9, 121.3, 115.3, 112.2, 109.8, 100.8, 55.6, 32.9. IR $\vee 3051(\mathrm{w}), 2946(\mathrm{w}), 1585$ (m), $1489(\mathrm{~s}), 1463(\mathrm{~m}), 1424$ (m), 1295 (w), $1266(\mathrm{~s})$, 
1228 (m), 1210 (s), 1181 (w), 1136 (m), 1088 (w), 1031 (m), 866 (m). HRMS (ESI) calcd for $\mathrm{C}_{21} \mathrm{H}_{19} \mathrm{~N}_{2} \mathrm{O}^{+}[\mathrm{M}+\mathrm{H}]^{+}$315.1492; found 315.1493.

\section{5-Fluoro-1-methyl-3-(2-(pyridin-2-yl)phenyl)-1H-indole (13e)}<smiles>Cn1cc(-c2ccccc2-c2ccccn2)c2cc(F)ccc21</smiles>

Starting from commercially available 2-phenylpyridine $12(43.0 \mu 1,0.300$ mmol) and with 1-(3-5-fluoro-1-methyl-1H-indole)-1H-1 $\lambda_{3}$-benzo[b]iodo$3(2 H)$-one $9 \mathbf{h}$ (130 mg, $0.330 \mathrm{mmol}, 1.10$ equiv.) at $50{ }^{\circ} \mathrm{C}, 5$-fluoro-1-methyl3-(2-(pyridin-2-yl)phenyl)-1H-indole 13e (66.0 mg, $0.218 \mathrm{mmol}, 73 \%$ yield) was obtained as a yellow oil. Rf: 0.40 (Pentane:EtOAc 4:1). ${ }^{1} \mathbf{H}$ NMR (400 $\left.\mathrm{MHz}, \mathrm{CDCl}_{3}\right) \delta 8.65(\mathrm{~d}, J=4.9 \mathrm{~Hz}, 1 \mathrm{H}, \mathrm{Ar} H), 7.71(\mathrm{~d}, J=7.2 \mathrm{~Hz}, 1 \mathrm{H}, \operatorname{Ar} H), 7.55(\mathrm{~d}, J=7.2 \mathrm{~Hz}$, 1H, ArH), $7.51-7.38(\mathrm{~m}, 2 \mathrm{H}), 7.33$ (t, $J=7.7 \mathrm{~Hz}, 1 \mathrm{H}, \mathrm{Ar} H), 7.17$ (dd, $J=9.0,4.3 \mathrm{~Hz}, 1 \mathrm{H}, \mathrm{Ar} H$ ), $7.08(\mathrm{t}, J=6.3 \mathrm{~Hz}, 1 \mathrm{H}, \operatorname{Ar} H), 7.08-6.97(\mathrm{~m}, 2 \mathrm{H}, \operatorname{Ar} H), 6.91$ (t, $J=9.0 \mathrm{~Hz}, 1 \mathrm{H}, \operatorname{Ar} H), 6.77(\mathrm{~s}, 1 \mathrm{H}$, $\mathrm{NCHC}), 3.70\left(\mathrm{~s}, 3 \mathrm{H}, \mathrm{NCH}_{3}\right) .{ }^{13} \mathbf{C}$ NMR $\left(101 \mathrm{MHz}, \mathrm{CDCl}_{3}\right) \delta 159.8,158.0(\mathrm{~d}, J=234.6 \mathrm{~Hz}), 149.3$, 139.7, 135.2, 133.4, 132.9, 130.8, 130.6, 129.7, 128.5, 127.3 (d, $J=9.9$ Hz), 126.9, 124.8, 121.3, 115.5 (d, $J=4.9 \mathrm{~Hz}), 110.0$ (d, $J=26.5 \mathrm{~Hz}), 109.7$ (d, $J=9.7 \mathrm{~Hz}), 104.7$ (d, $J=24.2 \mathrm{~Hz}), 33.0$. ${ }^{19}$ F NMR (376 MHz, $\left.\mathrm{CDCl}_{3}\right) \delta$-125.1. IR v 3063 (w), 2930 (w), 1624 (m), 1585 (m), 1488 (s), 1464 (m), 1425 (m), 1292 (w), 1192 (s), 1123 (m), 1060 (w), 873 (s). HRMS (ESI) calcd for $\mathrm{C}_{20} \mathrm{H}_{16} \mathrm{FN}_{2}{ }^{+}[\mathrm{M}+\mathrm{H}]^{+}$303.1292; found 303.1295

\section{5-Chloro-1-methyl-3-(2-(pyridin-2-yl)phenyl)-1 $H$-indole (13f)}

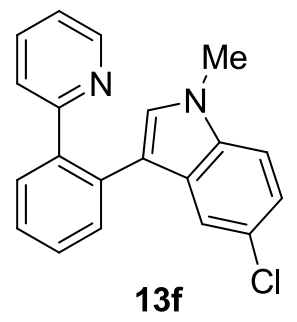

Starting from commercially available 2-phenylpyridine $12(43.0 \mu 1,0.300$ mmol) and with 1-(3-5-chloro-1-methyl- $1 H$-indole)-1H-1 $\lambda_{3}$-benzo[b]iodo$3(2 \mathrm{H})$-one $9 \mathbf{i}$ (136 mg, $0.330 \mathrm{mmol}, 1.10$ equiv.) at $50^{\circ} \mathrm{C}, 5$-fluoro-1-methyl-3(2-(pyridin-2-yl)phenyl)-1 $H$-indole $13 f$ (77.0 mg, $0.242 \mathrm{mmol}, 81 \%$ yield) was obtained as a yellow oil. Rf: 0.36 (Pentane:EtOAc 4:1). ${ }^{1} \mathbf{H}$ NMR (400 MHz, $\left.\mathrm{CDCl}_{3}\right) \delta 8.65(\mathrm{~m}, 1 \mathrm{H}, \mathrm{Ar} H), 7.72(\mathrm{dd}, J=7.3,1.8 \mathrm{~Hz}, 1 \mathrm{H}, \mathrm{Ar} H), 7.55$ (m, 1H, ArH), 7.46 (pd, $J$ $=7.3,1.8 \mathrm{~Hz}, 2 \mathrm{H}, \operatorname{Ar} H), 7.38-7.31(\mathrm{~m}, 2 \mathrm{H}, \operatorname{Ar} H), 7.17(\mathrm{~d}, J=8.6 \mathrm{~Hz}, 1 \mathrm{H}, \operatorname{Ar} H), 7.14-7.07(\mathrm{~m}$, 2H, $\operatorname{ArH}), 7.04(\mathrm{~m}, 1 \mathrm{H}, \mathrm{ArH}), 6.75(\mathrm{~s}, 1 \mathrm{H}, \mathrm{NCHC}), 3.69\left(\mathrm{~s}, 3 \mathrm{H}, \mathrm{NCH}_{3}\right) .{ }^{13} \mathbf{C}$ NMR $(101 \mathrm{MHz}$, $\left.\mathrm{CDCl}_{3}\right) \delta 159.6,149.0,139.5,135.6,135.2,132.7,131.0,130.6,129.4,128.7,128.0,127.1,125.5$, 
124.9, 121.9, 121.4, 119.3, 115.2, 110.2, 33.0. IR v 3055 (w), 3014 (w), 2924 (w), $1586(w), 1543$ (w), $1477(\mathrm{~s}), 1424(\mathrm{~m}), 1374(\mathrm{w}), 1288(\mathrm{w}), 1218(\mathrm{w}), 1152(\mathrm{w}), 1096(\mathrm{w}), 1058(\mathrm{w}), 953(\mathrm{w})$. HRMS (ESI) calcd for $\mathrm{C}_{20} \mathrm{H}_{16} \mathrm{ClN}_{2}{ }^{+}[\mathrm{M}+\mathrm{H}]^{+} 319.0997$; found 319.1006.

\section{5-Iodo-1-methyl-3-(2-(pyridin-2-yl)phenyl)-1H-indole (13g)}<smiles>Cn1cc(-c2ccccc2-c2ccccn2)c2cc(I)ccc21</smiles>

$13 \mathrm{~g}$

Starting from commercially available 2-phenylpyridine 12 (43.0 $\mu 1,0.300$ mmol) and with 1-(3-5-iodo-1-methyl-1H-indole)- $1 H-1 \lambda_{3}$-benzo[b]iodo$3(2 H)$-one $9 \mathbf{j}$ (166 mg, $0.330 \mathrm{mmol}, 1.10$ equiv.) at $50{ }^{\circ} \mathrm{C}, 5$-Iodo-1-methyl-3(2-(pyridin-2-yl)phenyl)-1 $H$-indole $\mathbf{1 3 g}$ (91.0 $\mathrm{mg}, 0.222 \mathrm{mmol}, 74 \%$ yield) was obtained as a yellow oil. Rf: 0.45 (Pentane:EtOAc 4:1). ${ }^{1} \mathbf{H}$ NMR (400 MHz, $\left.\mathrm{CDCl}_{3}\right) \delta 8.66(\mathrm{dd}, J=5.0,0.9 \mathrm{~Hz}, 1 \mathrm{H}, \mathrm{Ar} H), 7.71(\mathrm{~m}, 1 \mathrm{H}, \mathrm{Ar} H), 7.65(\mathrm{~d}, J=1.6 \mathrm{~Hz}, 1 \mathrm{H}, \mathrm{Ar} H)$, $7.53(\mathrm{~m}, 1 \mathrm{H}, \operatorname{Ar} H), 7.50-7.42(\mathrm{~m}, 2 \mathrm{H}, \operatorname{Ar} H), 7.39(\mathrm{dd}, J=8.5,1.6 \mathrm{~Hz}, 1 \mathrm{H}, \operatorname{Ar} H), 7.32(\mathrm{td}, J=$ 7.7, $1.9 \mathrm{~Hz}, 1 \mathrm{H}, \mathrm{Ar} H$ ), 7.08 (ddd, $J=7.7,5.0,1.1 \mathrm{~Hz}, 1 \mathrm{H}, \mathrm{Ar} H), 7.07-6.97$ (m, 2H, ArH), 6.70 (s, 1H, NCHC), 3.67 (s, 3H, NCH3). ${ }^{13} \mathbf{C}$ NMR (101 MHz, $\left.\mathrm{CDCl}_{3}\right) \delta 159.7,149.3,139.8,135.7$, 135.2, 132.5, 130.9, 130.5, 129.8, 129.2, 128.7, 128.7, 128.5, 127.0, 124.7, 121.4, 114.9, 111.1, 83.2, 32.9. IR $v 3058(\mathrm{w}), 2920(\mathrm{w}), 1606(\mathrm{w}), 1585$ (m), 1474 (s), $1422(\mathrm{~m}), 1371(\mathrm{w}), 1287(\mathrm{w})$, 1266 (w), 1217 (w), 1148 (w), 1092 (w), 1024 (w), 943 (w), 874 (w). HRMS (ESI) calcd for $\mathrm{C}_{20} \mathrm{H}_{16} \mathrm{IN}_{2}{ }^{+}[\mathrm{M}+\mathrm{H}]^{+}$411.0353; found 411.0348 .

\section{1-Methyl-3-(2-(pyridin-2-yl)phenyl)-5-(4,4,5,5-tetramethyl-1,3,2-dioxaborolan-2-yl)-1 H- indole (13h)}<smiles>Cn1cc(-c2ccccc2-c2ccccn2)c2cc(Br)ccc21</smiles>

$13 \mathrm{~h}$

Starting from commercially available 2-phenylpyridine $12(43.0 \mu 1,0.300$ mmol) and with 1-(3-1-methyl-5-(4,4,5,5-tetramethyl-1,3,2-dioxaborolan-2yl)-1H-indole)-1H-1 $\lambda_{3}$-benzo[b]iodo-3(2H)-one 9k (166 mg, $0.330 \mathrm{mmol}$, 1.10 equiv.) at $50{ }^{\circ} \mathrm{C}, 1-$ Methyl-3-(2-(pyridin-2-yl)phenyl)-5-(4,4,5,5tetramethyl-1,3,2-dioxaborolan-2-yl)-1H-indole $\mathbf{1 3 h}(73.0 \mathrm{mg}, 0.178 \mathrm{mmol}$, 59\% yield) was obtained as a yellow oil. Rf: 0.30 (Pentane:EtOAc 4:1). ${ }^{1} \mathbf{H}$ NMR (400 MHz, $\left.\mathrm{CDCl}_{3}\right) \delta 8.64(\mathrm{~d}, J=5.0 \mathrm{~Hz}, 1 \mathrm{H}, \mathrm{Ar} H), 8.00(\mathrm{~s}, 1 \mathrm{H}, \mathrm{Ar} H), 7.71(\mathrm{~d}, J=7.5 \mathrm{~Hz}, 1 \mathrm{H}, \mathrm{Ar} H), 7.64$ (dd, $J=8.3,1.1 \mathrm{~Hz}, 1 \mathrm{H}, \mathrm{Ar} H), 7.61(\mathrm{~m}, 1 \mathrm{H}, \mathrm{Ar} H), 7.46(\mathrm{td}, J=7.4,1.7 \mathrm{~Hz}, 1 \mathrm{H}, \mathrm{Ar} H), 7.41(\mathrm{td}, J=7.4$, $1.6 \mathrm{~Hz}, 1 \mathrm{H}, \mathrm{Ar} H), 7.29-7.26$ (m, 2H, $\operatorname{Ar} H), 7.00$ (ddd, $J=7.5,4.9,1.2 \mathrm{~Hz}, 1 \mathrm{H}, \operatorname{Ar} H), 6.95$ (dt, $J$ $=7.9,1.0 \mathrm{~Hz}, 1 \mathrm{H}, \operatorname{Ar} H), 6.62(\mathrm{~s}, 1 \mathrm{H}, \mathrm{Ar} H), 3.68(\mathrm{~s}, 3 \mathrm{H}, \mathrm{NCH}), 1.34(\mathrm{~s}, 12 \mathrm{H}, \mathrm{BPin}) .{ }^{13} \mathbf{C} \mathbf{~ N M R}$ 
$\left(101 \mathrm{MHz}, \mathrm{CDCl}_{3}\right) \delta 159.9,149.1,140.0,138.6,135.0,133.1,131.2,130.3,128.4,128.2,127.7$, 127.7, 126.9, 126.7, 124.9, 121.1, 116.0, 108.5, 83.3, 32.6, 24.8 (one aromatic Carbon signal not resolved). IR $v 3063(\mathrm{w}), 2978(\mathrm{w}), 2940(\mathrm{w}), 2245(\mathrm{w}), 2214(\mathrm{w}), 1608(\mathrm{~m}), 1605(\mathrm{~m}), 1568(\mathrm{w})$, 1463 (m), 1438 (m), 1383 (s), 1349 (s), 1311 (s), 1273 (m), 1142 (s), 1097 (m), 967 (m), 910 (s), 866 (m). HRMS (ESI) calcd for $\mathrm{C}_{26} \mathrm{H}_{28} \mathrm{BN}_{2} \mathrm{O}_{2}{ }^{+}[\mathrm{M}+\mathrm{H}]^{+} 411.2238$; found 411.2248.

\section{2-(2-(1-Methyl-1H-pyrrol-3-yl)phenyl)pyridine (13i)}

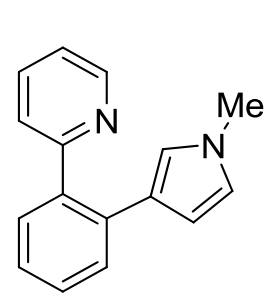

$13 \mathbf{i}$

Starting from commercially available 2-phenylpyridine $12(43.0 \mu 1,0.300 \mathrm{mmol}$, 1.00 equiv.) and with 1-(3-1-methyl- $1 H$-pyrrole)-1H-1 $1 \lambda_{3}$-benzo[b]iodo-3(2H)one 90 (108 mg, $0.330 \mathrm{mmol}, 1.10$ equiv.) at $80^{\circ} \mathrm{C}$, 2-(2-(1-Methyl- $1 H$-pyrrol-3yl)phenyl)pyridine $\mathbf{1 3 i}$ (60.0 $\mathrm{mg}, 0.256 \mathrm{mmol}, 85 \%$ yield) was obtained as a yellow oil. Rf: 0.38 (Pentane:EtOAc 4:1). ${ }^{1} \mathbf{H}$ NMR (400 MHz, $\left.\mathrm{CDCl}_{3}\right) \delta 8.67$ $(\mathrm{d}, J=5.0 \mathrm{~Hz}, 1 \mathrm{H}, \operatorname{Ar} H), 7.57-7.46$ (m, 3H, $\operatorname{Ar} H), 7.37$ (t, $J=7.5 \mathrm{~Hz}, 1 \mathrm{H}, \operatorname{Ar} H), 7.31$ (d, $J=7.5$ $\mathrm{Hz}, 1 \mathrm{H}, \operatorname{Ar} H), 7.22(\mathrm{~d}, J=7.9 \mathrm{~Hz}, 1 \mathrm{H}, \operatorname{Ar} H), 7.18(\mathrm{dd}, J=7.4,5.1 \mathrm{~Hz}, 1 \mathrm{H}, \operatorname{Ar} H), 6.43(\mathrm{t}, J=2.5$ $\mathrm{Hz}, 1 \mathrm{H}, \operatorname{Ar} H), 6.30(\mathrm{~d}, J=1.6 \mathrm{~Hz}, 1 \mathrm{H}, \mathrm{Ar} H), 5.80$ (d, $J=1.6 \mathrm{~Hz}, 1 \mathrm{H}, \mathrm{ArH}), 3.54\left(\mathrm{~s}, 3 \mathrm{H}, \mathrm{NCH}_{3}\right)$. ${ }^{13}$ C NMR $\left(101 \mathrm{MHz}, \mathrm{CDCl}_{3}\right) \delta 160.6,149.1,138.7,135.2,134.8,130.2,129.3,128.3,125.8$, 125.2, 123.8, 121.5, 121.3, 120.6, 109.2, 36.1. IR $v 3056(\mathrm{w}), 3007$ (w), $2943(\mathrm{w}), 1586(\mathrm{~s}), 1551$ (m), 1508 (m), 1463 (s), 1424 (s), 1361 (m), 1260 (w), 1202 (s), 1150 (w), 1087 (w), 1024 (w), $990(w), 926(w)$. HRMS (ESI) calcd for $\mathrm{C}_{16} \mathrm{H}_{15} \mathrm{~N}_{2}{ }^{+}[\mathrm{M}+\mathrm{H}]^{+}$235.1230; found 235.1230.

\section{2-(2-(1H-Pyrrol-3-yl)phenyl)pyridine (13j)}

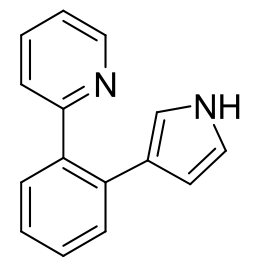

13j

Starting from commercially available 2-phenylpyridine $12(43.0 \mu 1,0.300 \mathrm{mmol}$, 1.00 equiv.) and with 1-(3-1H-pyrrole)- $1 H$ - $1 \lambda_{3}$-benzo[b]iodo-3(2H)-one 9n (103 $\mathrm{mg}, 0.330 \mathrm{mmol}, 1.10$ equiv.) in a mixture of 1,2-DCE:MeOH $(2: 1,0.1 \mathrm{M})$ at 50 ${ }^{\circ} \mathrm{C}$, 2-(2-(1H-pyrrol-3-yl)phenyl)pyridine 13j (52.0 mg, $0.236 \mathrm{mmol}, 79 \%$ yield) was obtained as a light brown foam. Rf: 0.42 (Pentane:EtOAc 4:1) ${ }^{\mathbf{1}} \mathbf{H}$ NMR (400 $\left.\mathrm{MHz}, \mathrm{CDCl}_{3}\right) \delta 8.67(\mathrm{~m}, 1 \mathrm{H}, \mathrm{Ar} H), 8.29(\mathrm{~m}, 1 \mathrm{H}, \mathrm{NH}), 7.57-7.47(\mathrm{~m}, 3 \mathrm{H}, \mathrm{Ar} H), 7.40(\mathrm{dd}, J=8.2$, $6.8 \mathrm{~Hz}, 1 \mathrm{H}, \operatorname{Ar} H), 7.32$ (t, $J=7.3 \mathrm{~Hz}, 1 \mathrm{H}, \operatorname{Ar} H), 7.23-7.15$ (m, 2H, $\operatorname{Ar} H), 6.62$ (d, $J=2.6 \mathrm{~Hz}$, $1 \mathrm{H}, \operatorname{Ar} H), 6.43(\mathrm{~d}, J=2.6 \mathrm{~Hz}, 1 \mathrm{H}, \operatorname{Ar} H), 5.95(\mathrm{dd}, J=2.9,1.6 \mathrm{~Hz}, 1 \mathrm{H}, \operatorname{Ar} H) .{ }^{13} \mathbf{C}$ NMR $(101 \mathrm{MHz}$, $\left.\mathrm{CDCl}_{3}\right) \delta 160.6,149.2,139.0,135.2,134.7,130.2,129.6,128.4,126.1,125.2,123.8,121.4,117.6$, 116.8, 109.3. IR $v 3191(\mathrm{~m}), 3054(\mathrm{~m}), 2928(\mathrm{w}), 1601(\mathrm{~s}), 1589$ (s), $1562(\mathrm{~m}), 1505$ (m), 1464 (s), 
1426 (s), 1267 (w), 1152 (w), 1078 (w), 1028 (m), 996 (w), 917 (w). HRMS (ESI) calcd for $\mathrm{C}_{15} \mathrm{H}_{13} \mathrm{~N}_{2}^{+}[\mathrm{M}+\mathrm{H}]^{+} 221.1073$; found 221.1077.

\section{2-(2-(1-Benzyl-1H-pyrrol-3-yl)phenyl)pyridine (13k)}

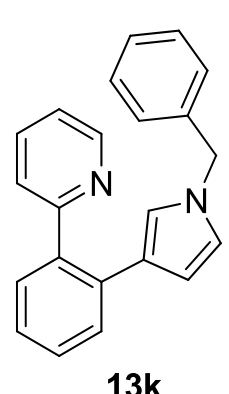

Starting from commercially available 2-phenylpyridine $12(43.0 \mu 1,0.300 \mathrm{mmol}$, 1.00 equiv.) and with 1 -(3-1-benzyl- $1 H$-pyrrole)- $1 H$ - $1 \lambda_{3}$-benzo[b]iodo-3(2H)-one 9q (133 mg, $0.330 \mathrm{mmol}, 1.10$ equiv.) at $80{ }^{\circ} \mathrm{C}, 2-(2-(1-B e n z y l-1 H-p y r r o l-3-$ yl)phenyl)pyridine 13k (54.0 mg, $0.174 \mathrm{mmol}, 58 \%$ yield) was obtained as an pale yellow oil. Rf: 0.30 (Pentane:EtOAc 4:1). ${ }^{1} \mathbf{H}$ NMR (400 MHz, $\left.\mathrm{CDCl}_{3}\right) \delta 8.62(\mathrm{~m}$, $1 \mathrm{H}, \operatorname{Ar} H), 7.51-7.45(\mathrm{~m}, 3 \mathrm{H}, \operatorname{Ar} H), 7.38(\mathrm{td}, J=7.5,1.6 \mathrm{~Hz}, 1 \mathrm{H}, \operatorname{Ar} H), 7.34-$ $7.26(\mathrm{~m}, 4 \mathrm{H}, \operatorname{Ar} H), 7.20(\mathrm{~d}, J=7.9 \mathrm{~Hz}, 1 \mathrm{H}, \operatorname{Ar} H) 7.13(\mathrm{ddd}, J=7.4,4.9,1.2 \mathrm{~Hz}$, 1H, $\operatorname{Ar} H), 7.03-6.97(\mathrm{~m}, 2 \mathrm{H}, \operatorname{Ar} H), 6.54(\mathrm{t}, J=2.5 \mathrm{~Hz}, 1 \mathrm{H}, \operatorname{Ar} H), 6.25$ (t, $J=1.8 \mathrm{~Hz}, 1 \mathrm{H}, \operatorname{Ar} H)$, $5.99(\mathrm{dd}, J=2.5,1.8 \mathrm{~Hz}, 1 \mathrm{H}, \mathrm{Ar} H), 4.92\left(\mathrm{~s}, 2 \mathrm{H}, \mathrm{NCH}_{2} \mathrm{Ph}\right) .{ }^{13} \mathbf{C ~ N M R}\left(101 \mathrm{MHz}, \mathrm{CDCl}_{3}\right) \delta 160.5$, $149.1,138.8,137.9,135.2,134.8,130.1,129.4,128.6,128.3,127.6,126.9,126.0,125.2,123.9$, 121.3, 121.0, 120.4, 109.4, 53.3. IR v $3062(\mathrm{~m}), 2925(\mathrm{~m}), 2854(\mathrm{w}), 1708(\mathrm{~m}), 1586(\mathrm{~s}), 1562(\mathrm{~m})$, 1498 (s), 1463 (s), 1425 (s), 1355 (m), 1190 (m), 1082 (m), 1025 (m). HRMS (ESI) calcd for $\mathrm{C}_{22} \mathrm{H}_{19} \mathrm{~N}_{2}^{+}[\mathrm{M}+\mathrm{H}]^{+}$311.1543; found 311.1545.

\section{2-(2-(1-methyl-1H-pyrrol-2-yl)phenyl)pyridine (13l)}

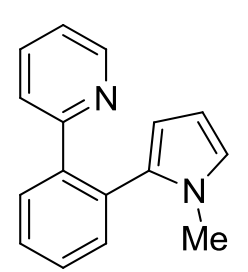

Starting from commercially available 2-phenylpyridine $12(43.0 \mu 1,0.300 \mathrm{mmol}$, 1.00 equiv.) and with 1-(2-1-methyl- $1 H$-pyrrole)- $1 H-1 \lambda_{3}$-benzo[ $\left.b\right]$ iodo-3(2H)-one 9p (108 mg, $0.330 \mathrm{mmol}, 1.10$ equiv.) at $80{ }^{\circ} \mathrm{C}, 2-(2-(1-\mathrm{methyl}-1 H$-pyrrol-2yl)phenyl)pyridine 131 (60.0 $\mathrm{mg}, 0.256 \mathrm{mmol}, 85 \%$ yield) was obtained as a pale 131 yellow oil. Rf: 0.42 (Pentane:EtOAc 4:1) ${ }^{1} \mathbf{H}$ NMR (400 MHz, $\left.\mathrm{CDCl}_{3}\right) \delta 8.67$ (m, $1 \mathrm{H}, \operatorname{Ar} H), 7.86(\mathrm{~d}, J=7.5 \mathrm{~Hz}, 1 \mathrm{H}, \operatorname{Ar} H), 7.55-7.40$ (m, 4H, ArH), 7.14 (ddd, $J=$ 7.5, 4.9, $1.1 \mathrm{~Hz}, 1 \mathrm{H}, \operatorname{Ar} H), 6.80(\mathrm{~m}, 1 \mathrm{H}, \operatorname{Ar} H), 6.51$ (t, $J=2.3 \mathrm{~Hz}, 1 \mathrm{H}, \operatorname{Ar} H), 6.18-6.15$ (m, 2H, $\mathrm{Ar} H), 2.90\left(\mathrm{~s}, 3 \mathrm{H}, \mathrm{NCH}_{3}\right) .{ }^{13} \mathbf{C}$ NMR $\left(101 \mathrm{MHz}, \mathrm{CDCl}_{3}\right) \delta 158.6,149.5,140.0,135.7,133.2,131.8$, 131.5, 130.1, 128.5, 128.4, 123.7, 122.2, 121.5, 109.2, 107.7, 33.8. IR $v 3098$ (w), 3059 (w), 2926 (m), 2854 (w), 1707 (w), 1585 (s), 1473 (s), 1429 (s), 1310 (s), 1239 (w), 1090 (m), 1056 (m), 1024 (m). HRMS (ESI) calcd for $\mathrm{C}_{16} \mathrm{H}_{15} \mathrm{~N}_{2}{ }^{+}[\mathrm{M}+\mathrm{H}]^{+}$235.1230; found 235.1233. 


\section{2-(2-(1-benzyl-1H-pyrrol-2-yl)phenyl)pyridine (13m)}

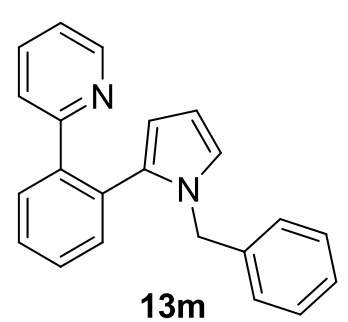

Starting from commercially available 2-phenylpyridine $\mathbf{1 2}(43.0 \mu 1,0.300$

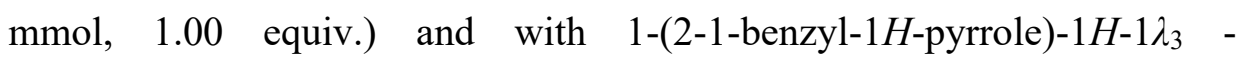
benzo[b]iodo-3(2H)-one 9r (133 mg, $0.330 \mathrm{mmol}, 1.10$ equiv.) at $80{ }^{\circ} \mathrm{C}, 2-$ (2-(1-benzyl-1H-pyrrol-2-yl)phenyl)pyridine 13m (63.0 mg, $0.203 \mathrm{mmol}$, $68 \%$ yield) as an pale yellow oil. Rf: 0.32 (Pentane:EtOAc 4:1). ${ }^{1} \mathbf{H}$ NMR $\left(400 \mathrm{MHz}, \mathrm{CDCl}_{3}\right) \delta 8.68(\mathrm{~d}, J=4.9 \mathrm{~Hz}, 1 \mathrm{H}, \mathrm{Ar} H), 7.85(\mathrm{~m}, 1 \mathrm{H}, \mathrm{Ar} H), 7.53$ - 7.42 (m, 2H, ArH), 7.42 - 7.33 (m, 2H, ArH), $7.17-7.13$ (m, 4H, $\operatorname{Ar} H), 6.83(\mathrm{~d}, J=7.9 \mathrm{~Hz}, 1 \mathrm{H}$, $\operatorname{Ar} H), 6.72(\mathrm{dd}, J=6.6,2.9 \mathrm{~Hz}, 2 \mathrm{H}, \operatorname{Ar} H), 6.52(\mathrm{~m}, 1 \mathrm{H}, \operatorname{Ar} H), 6.24-6.13$ (m, 2H, ArH), 4.45 (s, $\left.2 \mathrm{H}, \mathrm{NCH}_{2} \mathrm{Ph}\right) .{ }^{13} \mathbf{C}$ NMR $\left(101 \mathrm{MHz}, \mathrm{CDCl}_{3}\right) \delta 158.7,149.5,140.2,138.1,135.8,133.2,132.1$, 131.7, 130.3, 128.6, 128.6, 128.4, 127.3, 127.2, 124.2, 121.7, 110.1, 108.3, 50.6. (2 Cs overlapping at 121.7, shown by HSQC) IR v 3062 (m), 3029 (w), 2925 (w), 1585 (s), 1471 (s), 1427 (s), 1311 (m), 1298 (m), 1236 (m), 1153 (w), 1076 (m), 1024 (m), 989 (w). HRMS (ESI) calcd for $\mathrm{C}_{22} \mathrm{H}_{19} \mathrm{~N}_{2}{ }^{+}[\mathrm{M}+\mathrm{H}]^{+}$311.1543; found 311.1542.

\section{3-(5-Methoxy-2-(pyridin-2-yl)phenyl)-1-methyl-1H-indole (13n)}

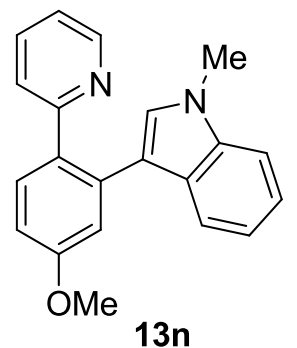

Starting from commercially available 2-(4-methoxyphenyl)pyridine (55.6 mg, $0.300 \mathrm{mmol})$ and with 1-(3-1-methyl- $1 H$-indole)- $1 H-1 \lambda_{3}$-benzo[b]iodo-3(2H)one $9 \mathrm{a}$ (124 mg, $0.330 \mathrm{mmol}, 1.10$ equiv.) at $50{ }^{\circ} \mathrm{C}, 3-(5$-methoxy-2-(pyridin2-yl)phenyl)-1-methyl-1H-indole 13n ( $83.0 \mathrm{mg}, 0.264 \mathrm{mmol}, 88 \%$ yield) was obtained as a yellow oil. Rf: 0.38 (Pentane:EtOAc 4:1). ${ }^{1} \mathbf{H}$ NMR (400 MHz, $\left.\mathrm{CDCl}_{3}\right) \delta 8.64(\mathrm{~d}, J=5.0 \mathrm{~Hz}, 1 \mathrm{H}, \mathrm{Ar} H), 7.70(\mathrm{~d}, J=8.5 \mathrm{~Hz}, 1 \mathrm{H}, \mathrm{Ar} H), 7.45(\mathrm{~d}$, $J=8.0 \mathrm{~Hz}, 1 \mathrm{H}, \operatorname{Ar} H), 7.31-7.23(\mathrm{~m}, 2 \mathrm{H}, \operatorname{Ar} H), 7.20(\mathrm{t}, J=7.6 \mathrm{~Hz}, 1 \mathrm{H}, \operatorname{Ar} H), 7.13(\mathrm{~d}, J=2.7 \mathrm{~Hz}$, 1H, $\mathrm{ArH}$ ), $7.06-6.95$ (m, 4H, $\mathrm{ArH}), 6.73$ (s, 1H, $\left.\mathrm{CH}_{3} \mathrm{NCHC}\right), 3.89$ (s, 3H, NCH3), 3.71 (s, 3H, $\left.\mathrm{OCH}_{3}\right) .{ }^{13} \mathbf{C}$ NMR $\left(101 \mathrm{MHz}, \mathrm{CDCl}_{3}\right) \delta 159.6,159.4,148.9,136.7,135.2,134.6,132.3,131.9$, $128.2,126.8,125.0,121.6,120.8,119.9,119.5,115.9,115.3,112.6,109.1,55.3,32.8$. IR v 3049 (w), $2933(\mathrm{w}), 2834$ (w), 1602 (s), 1568 (m), 1463 (s), 1426 (m), 1278 (s), 1232 (s), 1210 (m), 1062 (m), 1017 (m), 844 (w). HRMS (ESI) calcd for $\mathrm{C}_{21} \mathrm{H}_{19} \mathrm{~N}_{2} \mathrm{O}^{+}[\mathrm{M}+\mathrm{H}]^{+}$315.1492; found 315.1494. 


\section{3-(5-Chloro-2-(pyridin-2-yl)phenyl)-1-methyl-1 $H$-indole (130)}

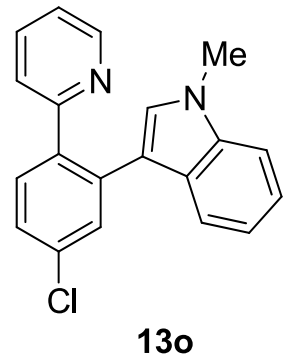

Starting from commercially available 2-(4-chlorophenyl)pyridine $(57.0 \mathrm{mg}$, $0.300 \mathrm{mmol})$ and with 1-(3-1-methyl-1H-indole)- $1 H-1 \lambda_{3}$-benzo[ $\left.b\right]$ iodo-3(2H)one 9a (124 mg, $0.330 \mathrm{mmol}, 1.10$ equiv.) at $50{ }^{\circ} \mathrm{C}, 3$-(5-chloro-2-(pyridin-2yl)phenyl)-1-methyl-1H-indole 130 ( $81.2 \mathrm{mg}, 0.255 \mathrm{mmol}, 85 \%$ yield) was obtained as a yellow oil. Rf: 0.40 (Pentane:EtOAc 4:1). ${ }^{1}$ H NMR (400 MHz, $\left.\mathrm{CDCl}_{3}\right) \delta 8.65(\mathrm{~m}, 1 \mathrm{H}, \mathrm{Ar} H), 7.68(\mathrm{~d}, J=8.3 \mathrm{~Hz}, 1 \mathrm{H}, \mathrm{Ar} H), 7.59(\mathrm{~d}, J=2.2 \mathrm{~Hz}$, $1 \mathrm{H}, \operatorname{Ar} H), 7.40(\mathrm{dd}, J=8.3,2.2 \mathrm{~Hz}, 1 \mathrm{H}, \operatorname{Ar} H), 7.37$ (dd, $J=8.0,1.0 \mathrm{~Hz}, 1 \mathrm{H}, \operatorname{Ar} H), 7.30$ (dd, $J=$ 7.7, $1.7 \mathrm{~Hz}, 1 \mathrm{H}, \operatorname{Ar} H), 7.27$ (m, 1H, ArH), 7.19 (ddd, $J=8.2,7.0,1.1 \mathrm{~Hz}, 1 \mathrm{H}, \operatorname{Ar} H), 7.08$ (ddd, $J$ $=7.5,4.9,1.1 \mathrm{~Hz}, 1 \mathrm{H}, \mathrm{ArH}), 7.04-6.99(\mathrm{~m}, 2 \mathrm{H}, \mathrm{ArH}), 6.75(\mathrm{~s}, 1 \mathrm{H}, \mathrm{NCHC}), 3.71\left(\mathrm{~s}, 3 \mathrm{H}, \mathrm{NCH}_{3}\right)$. ${ }^{13} \mathrm{C}$ NMR $\left(101 \mathrm{MHz}, \mathrm{CDCl}_{3}\right) \delta 158.7,149.2,137.9,136.8,135.4,135.1,134.3,132.0,130.7$, 128.4, 126.7, 126.6, 124.9, 121.8, 121.5, 119.8, 119.7, 114.3, 109.2, 32.8. IR v 3055 (w), $2926(w)$, 1595 (s), 1566 (m), 1476 (m), 1461 (s), 1427 (w), 1329 (m), 1219 (w), 1163 (w), 1100 (m), 1026 (w), 913 (w), 830 (w). HRMS (ESI) calcd for $\mathrm{C}_{20} \mathrm{H}_{16} \mathrm{ClN}_{2}{ }^{+}[\mathrm{M}+\mathrm{H}]^{+}$319.0997; found 319.0999.

\section{3-(5-Bromo-2-(pyridin-2-yl)phenyl)-1-methyl-1H-indole (13p)}

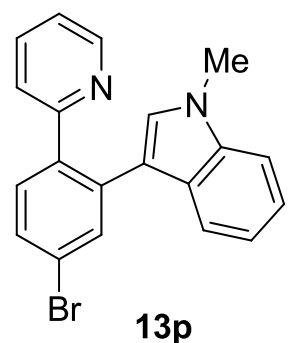

1H, $\operatorname{Ar} H), 7.56(\mathrm{dd}, \mathrm{J}=8.3,2.0 \mathrm{~Hz}, 1 \mathrm{H}, \operatorname{Ar} H), 7.36-7.31(\mathrm{~m}, 2 \mathrm{H}, \operatorname{Ar} H), 7.28(\mathrm{dt}, J=8.2,0.9 \mathrm{~Hz}$, 1H, ArH), $7.19(\mathrm{ddd}, \mathrm{J}=8.2,6.9,0.9 \mathrm{~Hz}, 1 \mathrm{H}, \operatorname{Ar} H), 7.11(\mathrm{t}, \mathrm{J}=6.3 \mathrm{~Hz}, 1 \mathrm{H}, \operatorname{Ar} H), 7.04(\mathrm{~d}, \mathrm{~J}=8.0$ $\mathrm{Hz}, 1 \mathrm{H}, \mathrm{ArH}), 6.99$ (m, 1H, ArH), 6.77 (s, 1H, NCHC), 3.72 (s, 3H, NCH3). ${ }^{13} \mathbf{C}$ NMR (101 MHz, $\left.\mathrm{CDCl}_{3}\right) \delta 158.7,149.3,138.5,136.7,135.4,135.2,133.5,132.1,129.6,128.3,126.5,124.7,122.4$, 121.8, 121.5, 119.7, 119.6, 114.2, 109.2, 32.7. IR $v 2956$ (w), 2929 (w), 2851 (w), 1678 (w), 1592 (m), 1510 (m), 1453 (m), 1453 (m), 1434 (s), 1399 (s), 1364 (s), 1264 (m), 1108 (m), 1031 (m), 956 (m). HRMS (ESI) calcd for $\mathrm{C}_{20} \mathrm{H}_{16}{ }^{79} \mathrm{BrN}_{2}{ }^{+}[\mathrm{M}+\mathrm{H}]^{+} 363.0491$; found 363.0490 


\section{Methyl 3-(1-methyl-1H-indol-3-yl)-4-(pyridin-2-yl)benzoate (13q)}

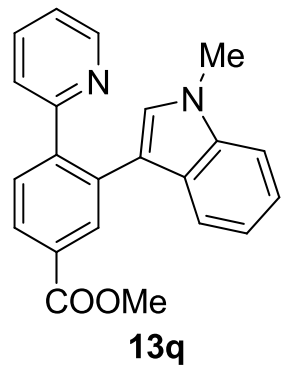

Starting from methyl 4-(pyridin-2-yl)benzoate $44(64.0 \mathrm{mg}, 0.300 \mathrm{mmol})$ and with 1-(3-1-methyl-1H-indole)-1H-1 $\lambda_{3}$-benzo[b]iodo-3(2H)-one 9a (124 mg, $0.330 \mathrm{mmol}, 1.10$ equiv.) at $50^{\circ} \mathrm{C}$, methyl 3-(1-methyl-1H-indol-3-yl)-4(pyridin-2-yl)benzoate 13q ( $94.0 \mathrm{mg}, 0.275 \mathrm{mmol}, 92 \%$ yield) was obtained as an orange oil. Rf: 0.33 (Pentane:EtOAc 4:1). ${ }^{1} \mathbf{H}$ NMR $\left(400 \mathrm{MHz}, \mathrm{CDCl}_{3}\right) \delta$ $8.61(\mathrm{dd}, J=5.1,1.6 \mathrm{~Hz}, 1 \mathrm{H}, \operatorname{Ar} H), 8.20(\mathrm{~d}, J=1.7 \mathrm{~Hz}, 1 \mathrm{H}, \operatorname{Ar} H), 7.99(\mathrm{dd}, J=8.1,1.8 \mathrm{~Hz}, 1 \mathrm{H}$, $\operatorname{Ar} H), 7.72(\mathrm{~d}, J=8.1 \mathrm{~Hz}, 1 \mathrm{H}, \operatorname{Ar} H), 7.26(\mathrm{~d}, J=8.0 \mathrm{~Hz}, 1 \mathrm{H}, \operatorname{Ar} H), 7.24-7.16(\mathrm{~m}, 2 \mathrm{H}, \mathrm{ArH}), 7.09$ (t, $J=7.6 \mathrm{~Hz}, 1 \mathrm{H}, \operatorname{Ar} H), 7.03-6.96(\mathrm{~m}, 2 \mathrm{H}, \operatorname{Ar} H), 6.90$ (t, $J=7.5 \mathrm{~Hz}, 1 \mathrm{H}, \operatorname{Ar} H), 6.71(\mathrm{~s}, 1 \mathrm{H}$, $\mathrm{NCHC}), 3.85$ (s, 3H, COOMe), 3.61 (s, $\left.3 \mathrm{H}, \mathrm{NCH}_{3}\right) .{ }^{13} \mathbf{C}$ NMR $\left(101 \mathrm{MHz}, \mathrm{CDCl}_{3}\right) \delta 167.0,158.8$, $149.4,143.8,136.8,135.3,133.7,132.3,130.7,129.9,128.3,127.6,126.7,124.7,121.8,121.7$, 119.7, 114.7, 109.1, 52.1, 32.8 (two aromatic Carbon signals overlapping at 119.7). IR v 3052 (w), 2950 (w), 2252 (w), 2218 (w), 1718 (s), 1587 (m), 1436 (m), 1365 (m), 1330 (m), 1286 (s), 1251 (s), 1162 (m), 1113 (m), 994 (w), 911 (s). HRMS (ESI) calcd for $\mathrm{C}_{22} \mathrm{H}_{19} \mathrm{~N}_{2} \mathrm{O}_{2}{ }^{+}[\mathrm{M}+\mathrm{H}]^{+}$343.1441; found 343.1438.

\section{3-(1-Methyl-1H-indol-3-yl)-4-(pyridin-2-yl)benzonitrile (13r)}

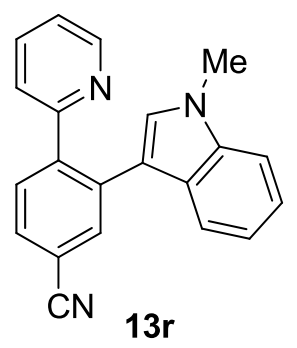

Starting from commercially available 4-(pyridin-2-yl)benzonitrile $(54.0 \mathrm{mg}$, $0.300 \mathrm{mmol})$ and with 1 -(3-1-methyl- $1 H$-indole)- $1 H$-1 $\lambda_{3}$-benzo[b]iodo-3(2H)one 9a (124 mg, $0.330 \mathrm{mmol}, 1.10$ equiv.) at $80{ }^{\circ} \mathrm{C}, 3$-(1-methyl- $1 H$-indol-3yl)-4-(pyridin-2-yl)benzonitrile $\mathbf{1 3 r}(72.0 \mathrm{mg}, 0.233 \mathrm{mmol}, 78 \%$ yield) was obtained as a yellow oil. Rf: 0.35 (Pentane:EtOAc 4:1). ${ }^{1} \mathbf{H}$ NMR $(400 \mathrm{MHz}$, $\left.\mathrm{CDCl}_{3}\right) \delta 8.70(\mathrm{~m}, 1 \mathrm{H}, \mathrm{Ar} H), 7.88(\mathrm{~d}, J=1.7 \mathrm{~Hz}, 1 \mathrm{H}, \operatorname{Ar} H), 7.83(\mathrm{~d}, J=8.0 \mathrm{~Hz}$, 1H, ArH), 7.69 (dd, $J=8.0,1.7 \mathrm{~Hz}, 1 \mathrm{H}, \operatorname{Ar} H), 7.36-7.27$ (m, 3H, $\operatorname{Ar} H$ ), 7.21 (ddd, $J=8.3,7.0$, $1.1 \mathrm{~Hz}, 1 \mathrm{H}, \operatorname{Ar} H$ ), 7.13 (ddd, $J=7.5,4.9,1.2 \mathrm{~Hz}, 1 \mathrm{H}, \operatorname{Ar} H$ ), 7.05 (d, $J=8.0 \mathrm{~Hz}, 1 \mathrm{H}, \operatorname{Ar} H), 7.02$ (ddd, $J=8.0,7.0,1.1 \mathrm{~Hz}, 1 \mathrm{H}, \operatorname{Ar} H), 6.77(\mathrm{~s}, 1 \mathrm{H}, \mathrm{NCHC}), 3.73(\mathrm{~s}, 3 \mathrm{H}, \mathrm{NCH}) .{ }^{13} \mathbf{C}$ NMR $(101$ $\left.\mathrm{MHz} \mathrm{CDCl}_{3}\right) \delta 157.9,149.6,143.6,136.7,135.6,134.8,134.5,131.5,129.8,128.4,126.3,124.7$, 122.2, 122.0, 120.1, 119.3, 118.9, 113.3, 112.1, 109.4, 32.9. IR v $3053(\mathrm{w}), 2932(\mathrm{w}), 2230(\mathrm{~m})$, 
$1587(\mathrm{~m}), 1558(\mathrm{w}), 1475(\mathrm{~m}), 1463(\mathrm{~m}), 1330(\mathrm{w}), 1266(\mathrm{~s}), 1224(\mathrm{w}), 1160(\mathrm{w}), 1091(\mathrm{w}), 1026$ (w), 902 (w), 839 (m). HRMS (ESI) calcd for $\mathrm{C}_{21} \mathrm{H}_{16} \mathrm{~N}_{3}{ }^{+}[\mathrm{M}+\mathrm{H}]^{+}$310.1339; found 310.1344.

\section{Methyl 3,5-bis(1-methyl-1H-indol-3-yl)-4-(pyridin-2-yl)benzoate (19)}

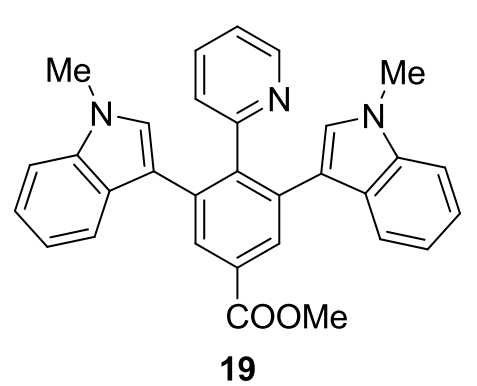

Starting from methyl 4-(pyridin-2-yl)benzoate 44 (64.0 mg, 0.300 mmol) and with 1-(3-1-methyl-1H-indole)-1H-1 $\lambda_{3}$-benzo[b]iodo$3(2 H)$-one 9a (249 mg, $0.660 \mathrm{mmol}, 2.20$ equiv.) at $50{ }^{\circ} \mathrm{C}$, methyl 3,5-bis(1-methyl-1H-indol-3-yl)-4-(pyridin-2-yl)benzoate 19 (75.0 $\mathrm{mg}, 0.159 \mathrm{mmol}, 53 \%$ yield) was obtained as a yellow oil. Rf: 0.30 (Pentane:EtOAc 4:1). ${ }^{1} \mathbf{H}$ NMR (400 MHz, $\left.\mathrm{CDCl}_{3}\right) \delta 8.47(\mathrm{~m}, 1 \mathrm{H}$, $\operatorname{Ar} H), 8.32$ (s, 2H, ArH), 7.68 (d, $J=8.0 \mathrm{~Hz}, 2 \mathrm{H}, \operatorname{Ar} H), 7.29-7.15$ (m, 5H, ArH), 7.08 (ddd, $J=$ 8.2, 6.5, $1.5 \mathrm{~Hz}, 2 \mathrm{H}, \mathrm{ArH}), 6.99$ - 6.93 (m, 2H, ArH), 6.52 (s, 2H, ArH), 3.94 (s, 3H, COOMe), $3.58(\mathrm{~s}, 6 \mathrm{H}, \mathrm{NMe}) .{ }^{13} \mathrm{C} \mathbf{N M R}\left(101 \mathrm{MHz}, \mathrm{CDCl}_{3}\right) \delta 167.2,159.8,147.9,143.2,136.4,135.6,135.4$, 129.7, 128.9, 127.3, 125.7, 121.6, 121.5, 119.8, 119.5, 114.5, 109.0, 52.1, 32.6. IR v $3056(\mathrm{w})$, 2950 (w), 2247 (w), 1720 (m), 1571 (m), 1477 (m), 1427 (m), 1325 (m), 1293 (s), 1247 (s), 1161 (m), 1119 (m), 1004 (m), 908 (s). HRMS (ESI) calcd for $\mathrm{C}_{31} \mathrm{H}_{25} \mathrm{~N}_{3} \mathrm{O}_{2}[\mathrm{M}+\mathrm{H}]^{+}$472.1998; found 472.2021 .

\section{1-Methyl-3-(2-(pyrimidin-2-yl)phenyl)-1H-indole (20)}

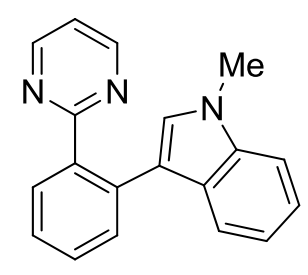

20

Starting from commercially available 2-phenylpyrimidine $(47.0 \mathrm{mg}, 0.300$ mmol) and with 1-(3-1-methyl-1H-indole)- $1 H$-1 $1 \lambda_{3}$-benzo[b]iodo-3(2H)-one 9a (124 mg, $0.330 \mathrm{mmol}, 1.10$ equiv.) at $80{ }^{\circ} \mathrm{C}, 1-$-methyl-3-(2-(pyrimidin-2yl)phenyl)- $1 H$-indole 20 (69.0 $\mathrm{mg}, 0.242 \mathrm{mmol}, 81 \%$ yield) was obtained as a yellow oil. Rf: 0.40 (Pentane:EtOAc 4:1). ${ }^{1} \mathbf{H}$ NMR (400 MHz, $\left.\mathrm{CDCl}_{3}\right) \delta 8.58$ (d, $J=4.9 \mathrm{~Hz}, 2 \mathrm{H}$, Pyrimidine $H$ ), 7.84 (dd, $J=7.7,1.5 \mathrm{~Hz}, 1 \mathrm{H}, \operatorname{Ar} H$ ), 7.59 (dd, $J=7.7,1.5 \mathrm{~Hz}$, $1 \mathrm{H}, \operatorname{Ar} H), 7.51$ (m, 1H, ArH), $7.44(\mathrm{td}, J=7.5,1.5 \mathrm{~Hz}, 1 \mathrm{H}, \operatorname{Ar} H), 7.24$ (d, $J=8.2 \mathrm{~Hz}, 1 \mathrm{H}, \operatorname{Ar} H)$, $7.10(\mathrm{dd}, J=9.3,7.5 \mathrm{~Hz}, 2 \mathrm{H}, \operatorname{Ar} H), 7.03$ (s, 1H, NCHCI), 7.00 (t, $J=4.9 \mathrm{~Hz}, 1 \mathrm{H}, \operatorname{Ar} H), 6.84(\mathrm{t}, J$ $=7.5 \mathrm{~Hz}, 1 \mathrm{H}, \mathrm{ArH}), 3.76\left(\mathrm{~s}, 3 \mathrm{H}, \mathrm{NCH}_{3}\right) .{ }^{13} \mathbf{C}$ NMR $\left(101 \mathrm{MHz}, \mathrm{CDCl}_{3}\right) \delta 168.5,156.7,138.1$, $136.7,134.1,131.3,130.7,129.4,127.5,126.8,126.5,121.3,119.3,119.2,118.2$, 116.2, 109.0, 
32.8 (two aromatic Carbon signals overlapping at 156.7). IR v 3048 (w), 2932 (w), 1599 (w), 1568 (s), $1554(\mathrm{~m}), 1473$ (w), 1414 (s), 1377 (w), 1330 (w), 1268 (w), $1221(\mathrm{w}), 1162(\mathrm{w}), 1135$ (w), 1015 (w), 943 (w), 822 (w). HRMS (ESI) calcd for $\mathrm{C}_{19} \mathrm{H}_{16} \mathrm{~N}_{3}{ }^{+}[\mathrm{M}+\mathrm{H}]^{+}$286.1339; found 286.1344.

\section{3-(2-(1H-Pyrazol-1-yl)phenyl)-1-methyl-1H-indole (21)}

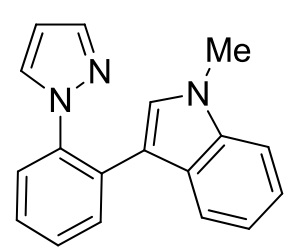

21

Starting from commercially available 1-phenyl- $1 H$-pyrazole $(43.0 \mathrm{mg}, 0.300$ mmol) and with 1-(3-1-methyl-1H-indole)-1H-1 $\lambda_{3}$-benzo[b]iodo-3(2H)-one 9a (124 mg, $0.330 \mathrm{mmol}, 1.10$ equiv.) at $80{ }^{\circ} \mathrm{C}, 3-(2-(1 H$-pyrazol-1-yl)phenyl)-1-

methyl- $1 H$-indole 21 (59.0 mg, $0.216 \mathrm{mmol}, 72 \%$ yield) was obtained as a yellow oil. Rf: 0.40 (Pentane:EtOAc 4:1). ${ }^{1} \mathbf{H}$ NMR (400 MHz, $\left.\mathrm{CDCl}_{3}\right) \delta 7.69$ (dd, J = 7.8, 1.6 Hz, 1H, ArH), 7.67 (d, J = 1.7 Hz, 1H, ArH), 7.62 (dd, J = 7.8, 1.6 Hz, 1H, ArH), $7.52-7.44(\mathrm{~m}, 2 \mathrm{H}, \operatorname{Ar} H), 7.40$ (td, J = 7.8, 1.6 Hz, 1H, ArH), 7.31 (d, J = 8.2 Hz, 1H, ArH), 7.25 - 7.20 (m, 2H, ArH), 7.07 (m, 1H, ArH), 6.60 (s, 1H, NCHC), 6.15 (m, 1H, ArH), 3.72 (s, 3H, $\left.\mathrm{NCH}_{3}\right) .{ }^{13} \mathrm{C}$ NMR $\left(101 \mathrm{MHz}, \mathrm{CDCl}_{3}\right) \delta 139.9,138.5,136.7,131.1,131.0,130.6,128.2,127.6$, 127.0, 126.9, 126.6, 121.8, 119.9, 119.3, 112.0, 109.3, 106.2, 32.8. IR $v 3049$ (w), 2926 (m), 2853 (w), $1680(\mathrm{w}), 1615(\mathrm{w}), 1604(\mathrm{w}), 1548(\mathrm{~m}), 1518$ (s), 1473 (s), $1423(\mathrm{w}), 1394$ (s), 1378 (s), 1329 (s), $1264(\mathrm{w}), 1221$ (m), 1089 (m), 1045 (s), 1019 (m), 936 (s). HRMS (ESI) calcd for $\mathrm{C}_{18} \mathrm{H}_{16} \mathrm{~N}_{3}{ }^{+}[\mathrm{M}+\mathrm{H}]^{+} 274.1339$; found 274.1343.

\section{0-(1-Methyl-1H-indol-3-yl)benzo[h]quinoline (22)}<smiles>Cn1cc(-c2cccc3ccc4cccnc4c23)c2ccccc21</smiles>

22

Starting from commercially available benzo[h]quinoline $(54.0 \mathrm{mg}, 0.300$ mmol) and with 1-(3-1-methyl-1H-indole)-1H-1 $\lambda_{3}$-benzo[b]iodo-3(2H)-one 9a (124 mg, $0.330 \mathrm{mmol}, 1.10$ equiv.) at $50{ }^{\circ} \mathrm{C}, 10-(1-$ methyl- $1 H$-indol-3yl)benzo[h]quinoline 22 (70.0 $\mathrm{mg}, 0.227 \mathrm{mmol}, 76 \%$ yield) was obtained as a light yellow oil. Rf: 0.40 (Pentane:EtOAc 4:1). ${ }^{1} \mathbf{H}$ NMR (400 MHz, $\left.\mathrm{CDCl}_{3}\right) \delta 8.27(\mathrm{~d}, J=2.4 \mathrm{~Hz}, 1 \mathrm{H}, \mathrm{Ar} H), 8.10(\mathrm{dd}, J=8.0,1.9 \mathrm{~Hz}, 1 \mathrm{H}, \operatorname{Ar} H), 7.93-7.90(\mathrm{~m}, 1 \mathrm{H}$, $\operatorname{Ar} H), 7.88(\mathrm{~d}, J=8.7 \mathrm{~Hz}, 1 \mathrm{H}, \operatorname{Ar} H), 7.75-7.65$ (m, 3H, $\operatorname{Ar} H), 7.37$ (d, $J=8.2 \mathrm{~Hz}, 1 \mathrm{H}, \operatorname{Ar} H), 7.29$ (m, 1H, ArH), $7.24(\mathrm{~m}, 1 \mathrm{H}, \operatorname{Ar} H), 7.16$ (ddd, $J=8.2,4.7,3.5 \mathrm{~Hz}, 1 \mathrm{H}, \operatorname{Ar} H), 6.79$ (s, 1H, NCHC), $6.78(\mathrm{t}, J=1.1 \mathrm{~Hz}, 1 \mathrm{H}, \mathrm{Ar} H), 3.93\left(\mathrm{~s}, 3 \mathrm{H}, \mathrm{NCH}_{3}\right) .{ }^{13} \mathbf{C} \mathbf{N M R}\left(101 \mathrm{MHz}, \mathrm{CDCl}_{3}\right) \delta 147.0,146.8$, 
137.0, 135.4, 135.3, 133.9, 132.8, 129.7, 128.5, 128.4, 127.8, 127.5, 127.4, 127.1, 126.2, 125.7, 121.0, 120.8, 120.7, 118.5, 108.9, 32.9. IR v 3046 (m), 2929 (m), 1677 (w), 1615 (w), $1588(\mathrm{~m})$, 1569 (m), 1475 (s), 1418 (s), 1375 (s), 1323 (s), 1263 (w), 1230 (s), 1161 (w), 1129 (w), 1014 (w), 910 (m). HRMS (ESI) calcd for $\mathrm{C}_{22} \mathrm{H}_{17} \mathrm{~N}_{2}{ }^{+}[\mathrm{M}+\mathrm{H}]^{+}$309.1386; found 309.1398.

\section{1'-Methyl-1-(pyrimidin-2-yl)-1 H,1'H-2,3'-biindole (23)}

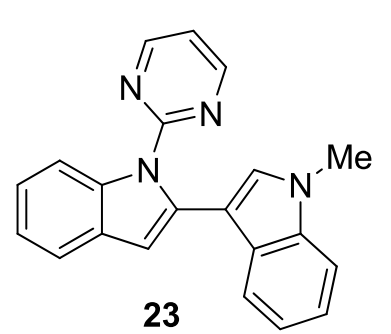

Starting from 1-(pyrimidin-2-yl)-1H-indole 46 (59.0 mg, $0.300 \mathrm{mmol})$ and with 1-(3-1-methyl-1H-indole)-1H-1 $\lambda_{3}$-benzo[b]iodo-3(2H)-one 9a (124 $\mathrm{mg}, 0.330 \mathrm{mmol}, 1.10$ equiv.) at $50{ }^{\circ} \mathrm{C}, 1$ '-methyl-1-(pyrimidin-2-yl)$1 H, 1^{\prime} H$-2,3'-biindole 23 ( $80.0 \mathrm{mg}, 0.247 \mathrm{mmol}, 82 \%$ yield) was obtained as a yellow oil. Rf: 0.38 (Pentane:EtOAc 4:1). ${ }^{1} \mathbf{H}$ NMR (400 MHz, $\left.\mathrm{CDCl}_{3}\right) \delta 8.51(\mathrm{~d}, J=4.8 \mathrm{~Hz}, 2 \mathrm{H}, \mathrm{Ar} H), 8.01(\mathrm{~m}, 1 \mathrm{H}, \mathrm{Ar} H), 7.56(\mathrm{dd}, J=$ 7.9, $1.3 \mathrm{~Hz}, 1 \mathrm{H}, \operatorname{Ar} H), 7.22-7.14$ (m, 3H, $\operatorname{Ar} H), 7.13$ (s, $1 \mathrm{H}, \operatorname{Ar} H), 7.06(\mathrm{dd}, J=8.3,6.9 \mathrm{~Hz}, 2 \mathrm{H}$, $\operatorname{Ar} H), 6.93$ (t, $J=4.8 \mathrm{~Hz}, 1 \mathrm{H}, \mathrm{ArH}), 6.82$ (td, $J=7.4,0.9 \mathrm{~Hz}, 1 \mathrm{H}, \mathrm{ArH}), 6.69$ (s, 1H, $\mathrm{CH}_{3} \mathrm{NCHC}$ ), $3.71\left(\mathrm{~s}, 3 \mathrm{H}, \mathrm{NCH}_{3}\right) .{ }^{13} \mathrm{C}$ NMR $\left(101 \mathrm{MHz}, \mathrm{CDCl}_{3}\right) \delta 158.2,158.2,137.5,136.8,134.6,129.6,127.8$, 126.8, 122.6, 121.8, 121.7, 120.0, 119.8, 119.5, 117.5, 112.4, 109.3, 108.9, 106.5, 32.9. IR v 3049 (w), $2931(\mathrm{w}), 1592(\mathrm{w}), 1563(\mathrm{~m}), 1454$ (m), 1423 (s), 1372 (w), 1350 (w), 1309 (m), $1258(\mathrm{w})$, 1217 (w), 910 (w). HRMS (ESI) calcd for $\mathrm{C}_{21} \mathrm{H}_{17} \mathrm{~N}_{4}^{+}[\mathrm{M}+\mathrm{H}]^{+}$325.1448; found 325.1452.

\section{8-(1-Methyl-1H-indol-3-yl)quinolin-2(1H)-one (24)}

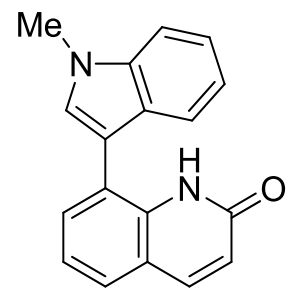

24

Starting from commercially available quinoline 1 -oxide $(44.0 \mathrm{mg}, 0.300 \mathrm{mmol})$ and with 1-(3-1-methyl-1H-indole)- $1 H-1 \lambda_{3}$-benzo[b]iodo-3(2H)-one 9a (124 $\mathrm{mg}, 0.330 \mathrm{mmol}, 1.10$ equiv.) at $100{ }^{\circ} \mathrm{C}, 8$-(1-methyl- $1 H$-indol-3-yl)quinolin2(1H)-one 24 (31.0 mg, $0.113 \mathrm{mmol}, 38 \%$ yield) was obtained as a yellow oil. Rf: 0.58 (EtOAc:MeOH 10:1). ${ }^{1} \mathbf{H}$ NMR $\left(400 \mathrm{MHz}, \mathrm{CDCl}_{3}\right) \delta 9.02(\mathrm{~s}, 1 \mathrm{H}$, $\mathrm{NHCO}), 7.83(\mathrm{~d}, J=9.5 \mathrm{~Hz}, 1 \mathrm{H}, \operatorname{Ar} H), 7.61-7.54$ (m, 2H, ArH), 7.45 (dd, $J$ = 8.3, 6.7 Hz, 2H, ArH), $7.34(\mathrm{~m}, 1 \mathrm{H}, \operatorname{Ar} H), 7.30(\mathrm{~d}, J=7.6 \mathrm{~Hz}, 1 \mathrm{H}, \operatorname{Ar} H), 7.23(\mathrm{~s}, 1 \mathrm{H}, \mathrm{NCHC})$, $7.20-7.15(\mathrm{~m}, 1 \mathrm{H}$, Indole $H), 6.66(\mathrm{~d}, J=9.3 \mathrm{~Hz}, 1 \mathrm{H}, \mathrm{Ar} H), 3.91(\mathrm{~s}, 3 \mathrm{H}, \mathrm{NCH}) .{ }^{13} \mathbf{C}$ NMR $(101$ $\left.\mathrm{MHz}, \mathrm{CDCl}_{3}\right) \delta 162.4,141.0,136.3,132.2,127.8,127.7,126.9,126.7,122.9,122.4,121.9,121.6$, 
120.6, 120.0, 119.4, 109.8, 109.4, 33.1. IR v 3367 (w), 3053 (w), 2923 (m), $2853(\mathrm{w}), 1715$ (w), 1651 (s), 1608 (m), 1541 (w), 1467 (m), 1372 (w), 1333 (w), 1234 (w), $1135(\mathrm{w}), 1014$ (w), 840 (m). HRMS (ESI) calcd for $\mathrm{C}_{18} \mathrm{H}_{15} \mathrm{~N}_{2} \mathrm{O}^{+}[\mathrm{M}+\mathrm{H}]^{+} 275.1179$; found 275.1185 .

(2R,3R,4R,5R)-2-(Acetoxymethyl)-5-(6-(2-(1-methyl-1H-indol-3-yl)phenyl)-9H-purin-9yl)tetrahydrofuran-3,4-diyl diacetate (25)<smiles></smiles>

Starting from $(2 R, 3 R, 4 R, 5 R)$-2-(acetoxymethyl)-5-(6-phenyl- $9 H$ purin-9-yl)tetrahydrofuran-3,4-diyl diacetate $\mathbf{5 0}$ (136 mg, 0.300 mmol) and with 1-(3-1-methyl- $1 H$-indole)-1H-1 $\lambda_{3}$-benzo[b]iodo3(2H)-one 9a (124 mg, $0.330 \mathrm{mmol}, 1.10$ equiv.) at $50{ }^{\circ} \mathrm{C}$, $(2 R, 3 R, 4 R, 5 R)-2-($ Acetoxymethyl)-5-(6-(2-(1-methyl-1H-indol3-yl)phenyl)-9H-purin-9-yl)tetrahydrofuran-3,4-diyl diacetate 25 (72 $\mathrm{mg}, 0.123 \mathrm{mmol}, 41 \%$ yield)) was obtained as a yellow oil. Rf: 0.65 (EtOAc 100\%). ${ }^{1} \mathbf{H}$ NMR $\left(400 \mathrm{MHz}, \mathrm{CDCl}_{3}\right) \delta 8.81(\mathrm{~s}, 1 \mathrm{H}, \mathrm{ArH}), 8.04$ (s, 1H, $\left.\mathrm{ArH}\right), 7.76$ $(\mathrm{dd}, J=7.6,1.4 \mathrm{~Hz}, 1 \mathrm{H}, \operatorname{Ar} H), 7.70(\mathrm{dd}, J=7.6,1.3 \mathrm{~Hz}, 1 \mathrm{H}, \operatorname{Ar} H), 7.56(\mathrm{td}, J=7.6,1.5 \mathrm{~Hz}, 1 \mathrm{H}$, $\operatorname{Ar} H), 7.46(\mathrm{td}, J=7.5,1.3 \mathrm{~Hz}, 1 \mathrm{H}, \operatorname{Ar} H), 7.30-7.23$ (m, $\left.1 \mathrm{H}, \mathrm{Ar} H+C D C l_{3}\right), 7.18$ (d, $J=8.3 \mathrm{~Hz}$, 1H, ArH), 7.08 (ddd, $J=8.1,7.0,1.1 \mathrm{~Hz}, 1 \mathrm{H}, \mathrm{Ar} H), 6.90$ (s, 1H, $\operatorname{Ar} H), 6.86$ (ddd, $J=8.0,6.9,1.0$ $\mathrm{Hz}, 1 \mathrm{H}, \mathrm{Ar} H), 6.18(\mathrm{~d}, J=5.1 \mathrm{~Hz}, 1 \mathrm{H}, \mathrm{CH}), 5.89$ (t, $J=5.3 \mathrm{~Hz}, 1 \mathrm{H}, \mathrm{CH}), 5.65(\mathrm{dd}, J=5.5,4.5 \mathrm{~Hz}$, $1 \mathrm{H}, \mathrm{CH}), 4.47-4.40\left(\mathrm{~m}, 2 \mathrm{H}, \mathrm{CH}+\mathrm{CH}_{2}\right), 4.34\left(\mathrm{dd}, J=13.1,5.2 \mathrm{~Hz}, 1 \mathrm{H}, \mathrm{CH}_{2}\right), 3.67(\mathrm{~s}, 3 \mathrm{H}, \mathrm{NMe})$, $2.14\left(\mathrm{~s}, 3 \mathrm{H}, \mathrm{COCH}_{3}\right), 2.08\left(\mathrm{~s}, 3 \mathrm{H}, \mathrm{COCH}_{3}\right), 2.06\left(\mathrm{~s}, 3 \mathrm{H}, \mathrm{COCH}_{3}\right) .{ }^{13} \mathbf{C ~ N M R}\left(101 \mathrm{MHz}, \mathrm{CDCl}_{3}\right) \delta$ $170.3,169.5,169.2,160.3,152.3,151.0,142.4,136.6,134.7,134.2,132.9,131.3,131.0,129.8$, $128.2,126.9,126.2,121.4,119.5,119.2,115.4,109.0,86.3,80.2,73.0,70.5,62.9,32.7,20.7,20.5$, 20.4. IR $v 3056$ (w), 2934 (w), 2825 (w), 2254 (w), 1749 (s), 1586 (m), 1505 (w), 1484 (w), 1435 (w), 1377 (m), 1332 (m), 1219 (s), 1101 (m), 1048 (m), 912 (m), 817 (w). HRMS (ESI) calcd for $\mathrm{C}_{31} \mathrm{H}_{30} \mathrm{~N}_{5} \mathrm{O}_{7}^{+}[\mathrm{M}+\mathrm{H}]^{+}$584.2140; found 584.2138. 
<smiles>Cc1c(I)c2ccccc2n1C</smiles>

$9 f$<smiles>C=CCCn1cc(I)c2ccccc21</smiles>

$9 c$

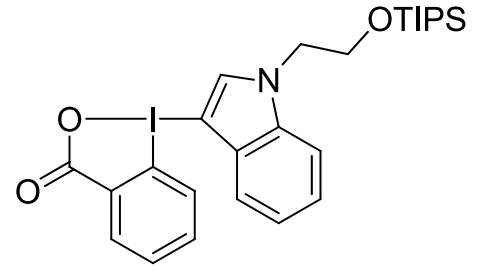

9d<smiles>Cn1cc(I2COC(=O)c3ccccc32)c2cccnc21</smiles>

9l<smiles>O=C1OI(c2cccs2)c2ccccc21</smiles>

9s<smiles>Cn1c2ccccc2c2cc(I3COC(=O)c4ccccc43)ccc21</smiles>

$9 m$

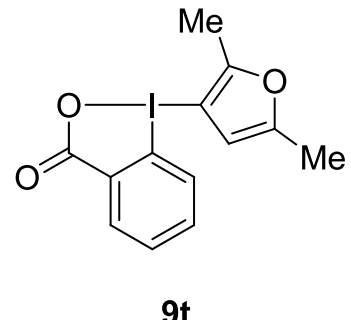

Figure S3: Reagents found ineffective for Rh-catalyzed CH activation. 


\section{Ru-Catalyzed C-H Indolization of Arenes via C-H activation.}

\subsection{Preparation of starting materials for Ru-Catalyzed $\mathrm{C}-\mathrm{H}$ activation.}<smiles>O=C(Cl)c1c[R1]ccc1</smiles>
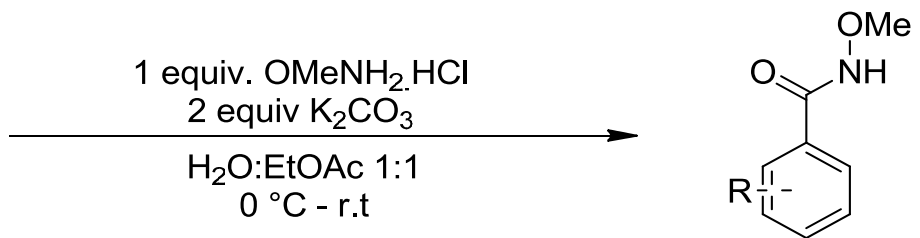

GP5: Following a reported procedure,$^{30}$ O-methylhydroxylamine hydrochloride $(0.418 \mathrm{~g}, 5.00$ mmol, 1.00 equiv.) was added to a solution of $\mathrm{K}_{2} \mathrm{CO}_{3}(1.38 \mathrm{~g}, 10.0 \mathrm{mmol}, 2.00$ equiv) in a mixture of EtOAc/ $\mathrm{H}_{2} \mathrm{O}(20 \mathrm{~mL}, 1: 10.25 \mathrm{M})$ under vigorous stirring. Then the reaction mixture was cooled to $0{ }^{\circ} \mathrm{C}$ and the corresponding (substituted) benzoyl chloride (5.00 mmol, 1.00 equiv) was added dropwise or portionwise. The reaction mixture was warmed to room temperature and stirred for additional 2 hours. The organic layer was separated and the aqueous layer was extracted with EtOAc $(3 \times 10 \mathrm{~mL})$. The combined organic layers were dried over $\mathrm{MgSO}_{4}$, filtered, and the solvent removed under reduced pressure. The crude product was purified by flash column chromatography (Pentane:EtOAc 2:1) to afford the desired N-methoxy benzamides 14-62.

[30] Y. Fukui, P. Liu, Q. Liu, Z.-T. He, N.-Y. Wu, P. Tian, G.-Q. Lin, J. Am. Chem. Soc. 2014, 136, $15607-15614$. 
<smiles>CONC(=O)c1ccccc1</smiles>

14<smiles>CONC(=O)c1ccc(Br)cc1</smiles>

56<smiles>CONC(=O)c1cccc(Cl)c1</smiles>

60<smiles>CONC(=O)c1ccc(C)cc1</smiles>

53<smiles>CONC(=O)c1cccc(C)c1</smiles>

57<smiles>CONC(=O)c1ccc2c(c1)OCO2</smiles>

61<smiles>CONC(=O)c1ccc(OC)cc1</smiles>

54<smiles>CONC(=O)c1cccc(C(F)(F)F)c1</smiles>

58

59

Figure S4: Starting materials for Ru-catalyzed Indolization of Arenes via C-H activation..

\section{N-Methoxybenzamide (14)}

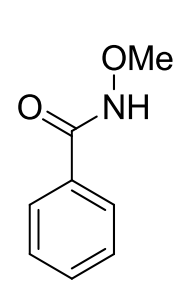

14

The synthesis of N-methoxybenzamide 14 was scaled up to $10 \mathrm{mmol}$ without reoptimization of the protocol.

Starting from commercially available benzoyl chloride $(1.15 \mathrm{~mL}, 10.0 \mathrm{mmol}), \mathrm{N}-$ methoxybenzamide 14 (1.20 g, $7.94 \mathrm{mmol}, 79 \%$ yield) was obtained as a colorless solid. ${ }^{1} \mathbf{H}$ NMR (400 MHz, $\left.\mathrm{CDCl}_{3}\right) \delta 10.70\left(\mathrm{~s}, 1 \mathrm{H}, \mathrm{NHOCH}_{3}\right), 7.70$ (dd, $J=8.3,1.4$ $\mathrm{Hz}, 2 \mathrm{H}, \operatorname{Ar} H), 7.38(\mathrm{~m}, 1 \mathrm{H}, \mathrm{Ar} H), 7.26(\mathrm{ddd}, J=8.3,6.6,1.3 \mathrm{~Hz}, 2 \mathrm{H}, \mathrm{Ar} H), 3.69$ (s, 3H, NHOCH 3 ). ${ }^{13}$ C NMR (100 MHz, $\left.\mathrm{CDCl}_{3}\right) \delta 166.2,131.7,128.3,127.1,63.9,53.3$. IR $v 3197(\mathrm{w}), 2980(\mathrm{w})$, 
2935 (w), 1646 (s), 1579 (m), 1516 (m), 1484 (m), 1310 (m), 1154 (w), 1045 (m), 1026 (m), 945 $(\mathrm{w}), 881(\mathrm{~s})$. NMR values are in accordance with the data reported in literature. ${ }^{30}$

\section{N-Methoxy-4-methylbenzamide (53)}

OMe Starting from commercially available 4-methylbenzoyl chloride (775 mg, $5.00 \mathrm{mmol}$ ), O NH N-methoxy-4-methylbenzamide 53 (822 mg, $4.98 \mathrm{mmol}, 100 \%$ yield) was obtained as a colorless solid. ${ }^{1} \mathbf{H}$ NMR (400 MHz, $\left.\mathrm{CDCl}_{3}\right) \delta 10.73$ (brs, $1 \mathrm{H}, \mathrm{NHOCH}_{3}$ ), 7.67 (d, $J$ $=8.2 \mathrm{~Hz}, 2 \mathrm{H}, \mathrm{ArH}), 7.10(\mathrm{~d}, J=8.0 \mathrm{~Hz}, 2 \mathrm{H}, \mathrm{ArH}), 3.74\left(\mathrm{~s}, 3 \mathrm{H}, \mathrm{NHOCH}_{3}\right), 2.30(\mathrm{~s}$, $\left.3 \mathrm{H}, \mathrm{ArCH}_{3}\right) .{ }^{13} \mathbf{C}$ NMR (100 MHz, $\left.\mathrm{CDCl}_{3}\right) \delta$ (ppm) 166.2, 142.1, 128.9, 127.1, 63.8,

$53 \quad 21.2$ (2 aromatic Carbon signals overlapping at 128.9). IR $v 3201(\mathrm{w}), 2975(\mathrm{w}), 2935$ (w), 1646 (s), 1572 (w), 1494 (m), 1439 (w), 1308 (m), 1155 (w), 1043 (s), 1020 (m), 943 (w), 883 $(\mathrm{s}), 833(\mathrm{~m})$. NMR values are in accordance with the data reported in literature. ${ }^{30}$

\section{N,4-dimethoxybenzamide (54)}

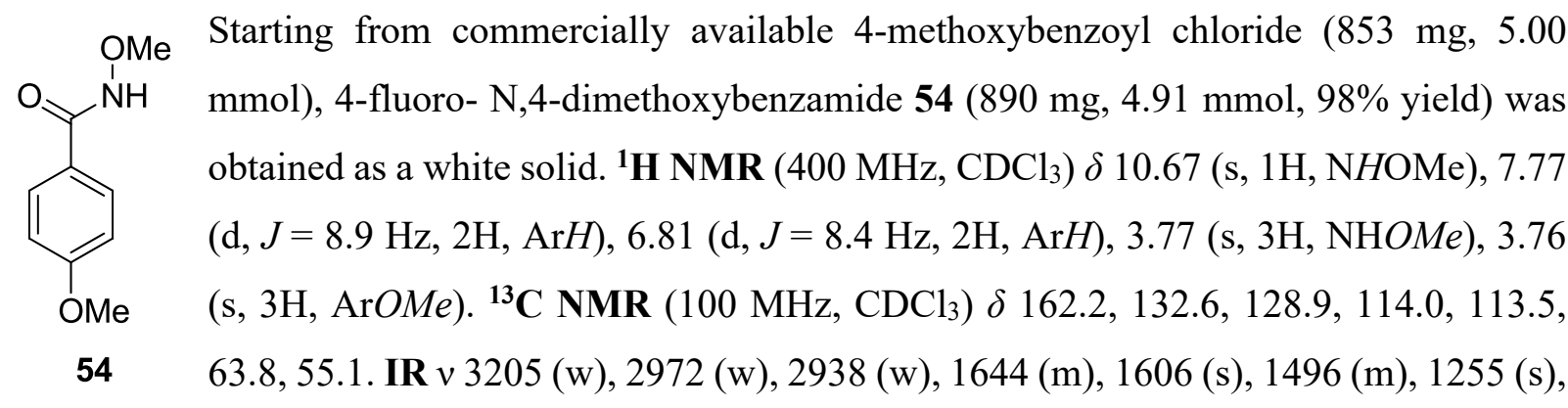
$1181(\mathrm{~m}), 1159(\mathrm{~m}), 1027(\mathrm{~s}), 884(\mathrm{~m}), 844(\mathrm{~m})$. NMR values are in accordance with the data reported in literature. ${ }^{30}$

\section{4-Fluoro-N-methoxybenzamide (55)}<smiles>CONC(=O)c1ccc(F)cc1</smiles>

55

Starting from commercially available 4-fluorobenzoyl chloride (793 mg, $5.00 \mathrm{mmol}$ ), 4-fluoro-N-methoxybenzamide 55 (643 $\mathrm{mg}, 3.80 \mathrm{mmol}, 76 \%$ yield) was obtained as a colorless solid. ${ }^{1} \mathbf{H}$ NMR (400 MHz, $\left.\mathrm{CDCl}_{3}\right) \delta 11.29$ (s, 1H, NHOMe), 7.78 (m, 2H, $\mathrm{ArH}), 6.96(\mathrm{~m}, 2 \mathrm{H}, \mathrm{ArH}), 3.72$ (s, 3H, NHOMe). ${ }^{13} \mathbf{C}$ NMR (101 MHz, $\left.\mathrm{CDCl}_{3}\right)$ 165.2, 164.7 (d, $J=252.5 \mathrm{~Hz}), 129.6$ (d, $J=9.0 \mathrm{~Hz}), 127.6$ (d, $J=3.3 \mathrm{~Hz}), 115.3$ (d, $J=22.0$ 
Hz), 63.7. IR $v 3210$ (w), 2984 (w), 2941 (w), 1656 (s), 1592 (s), 1481 (s), 1439 (w), 1318 (w), $1154(\mathrm{w}), 1072(\mathrm{~s}), 1012$ (s), $944(\mathrm{w}), 879(\mathrm{~s}), 841(\mathrm{~m})$. NMR data is corresponding to the reported values. $^{30}$

\section{4-Bromo-N-methoxybenzamide (56)}

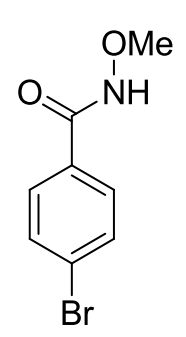

56

Starting from commercially available 4-bromobenzoyl chloride (1.09 g, $5.00 \mathrm{mmol})$, 4-bromo-N-methoxybenzamide 56 (700 mg, $3.04 \mathrm{mmol}, 61 \%$ yield) was obtained as a colorless solid. ${ }^{1} \mathbf{H}$ NMR $\left(400 \mathrm{MHz}, \mathrm{CDCl}_{3}\right) \delta 10.59$ (brs, $\left.1 \mathrm{H}, \mathrm{NHOCH}_{3}\right), 7.56$ (d, $J=$ $8.5 \mathrm{~Hz}, 2 \mathrm{H}, \mathrm{ArH}), 7.41(\mathrm{~d}, J=8.6 \mathrm{~Hz}, 2 \mathrm{H}), 3.71\left(\mathrm{~s}, 3 \mathrm{H}, \mathrm{NHOCH}_{3}\right) .{ }^{13} \mathbf{C}$ NMR (100 $\left.\mathrm{MHz}, \mathrm{CDCl}_{3}\right) \delta 165.4,131.7,130.4,128.8,126.7,64.1$. IR $v 3059(\mathrm{w}), 3006(\mathrm{w}), 1647$ NMR data is corresponding to the reported values. ${ }^{30}$

\section{N-Methoxy-3-methylbenzamide (57)}<smiles>CONC(=O)c1cccc(C)c1</smiles>

57

Starting from commercially available 3-methylbenzoyl chloride (775 mg, 5.00 mmol), N-methoxy-3-methylbenzamide 57 (813 mg, $4.92 \mathrm{mmol}, 98 \%$ yield) was obtained as a white solid. ${ }^{1} \mathbf{H}$ NMR $\left(400 \mathrm{MHz}, \mathrm{CDCl}_{3}\right) \delta(\mathrm{ppm}) 11.09$ (s, 1H, $\left.\mathrm{NHOCH}_{3}\right), 8.37(\mathrm{~s}, 1 \mathrm{H}, \mathrm{Ar} H), 8.03(\mathrm{dt}, J=7.8,1.5 \mathrm{~Hz}, 1 \mathrm{H}), 7.98(\mathrm{dt}, J=7.8,1.5$ $\mathrm{Hz}, 1 \mathrm{H}), 7.39$ (t, $J=7.8 \mathrm{~Hz}, 1 \mathrm{H}), 3.78\left(\mathrm{~s}, 6 \mathrm{H}, \mathrm{NHOMe}+\mathrm{ArCH}_{3}\right) .{ }^{13} \mathbf{C}$ NMR (101 $\left.\mathrm{MHz}, \mathrm{CDCl}_{3}\right) \delta 166.0,132.4,132.0,131.6,130.1,128.5,128.1,63.9,52.1 . \mathbf{I R} v 3004(\mathrm{w}), 2953$ (w), $1726(\mathrm{~s}), 1650(\mathrm{~m}), 1516(\mathrm{w}), 1439(\mathrm{~m}), 1303$ (m), 1264 (s), $1156(\mathrm{~m}), 1109(\mathrm{w}), 982$ (w), 826 (w). NMR values are in accordance with the data reported in literature. ${ }^{30}$

\section{N-Methoxy-3-(trifluoromethyl)benzamide (58)}

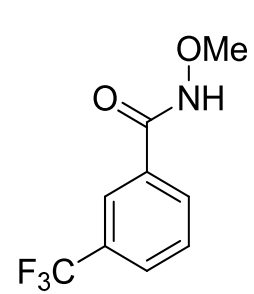

58

Starting from commercially available 3-(trifluoromethyl)benzoyl chloride (1.04 g, 5.00 mmol), N-methoxy-4-methylbenzamide 58 (822 mg, 3.75 mmol, 75\% yield) was obtained as a colorless solid. ${ }^{1} \mathbf{H}$ NMR (400 MHz, $\left.\mathrm{CDCl}_{3}\right) \delta 9.60$ (s, 1H, $\left.\mathrm{NHOCH}_{3}\right), 8.02(\mathrm{~s}, 1 \mathrm{H}, \mathrm{ArH}), 7.95(\mathrm{~d}, \mathrm{~J}=8.1 \mathrm{~Hz}, 1 \mathrm{H}, \mathrm{ArH}), 7.56(\mathrm{t}, \mathrm{J}=7.8$ $\mathrm{Hz}, 1 \mathrm{H}, \mathrm{Ar} H$ ), 3.87 (s, 3H, $\left.\mathrm{NHOCH}_{3}\right) .{ }^{13} \mathbf{C}$ NMR $\left(100 \mathrm{MHz}, \mathrm{CDCl}_{3}\right) \delta 168.3$, 
130.5, 129.3, 128.7, 128.6, 124.2, 124.2, $123.5\left(\mathrm{q}, \mathrm{J}_{\mathrm{F}}=272.6 \mathrm{~Hz}\right), 64.6 . \mathbf{I R} v 3190(\mathrm{w}), 2995(\mathrm{w})$, 1656 (m), 1522 (w), 1440 (w), 1330 (s), 1283 (m), 1169 (s), 1125 (s), 1075 (m), 909 (w). NMR values are in accordance with the data reported in literature. ${ }^{31}$

\section{3-Fluoro-N-methoxybenzamide (59)}<smiles>CONC(=O)c1cccc(F)c1</smiles>

59

Starting from commercially available 3-fluorobenzoyl chloride (775 mg, 5.00 mmol), 3-fluoro-N-methoxybenzamide 59 (710 $\mathrm{mg}, 4.20 \mathrm{mmol}, 84 \%$ yield) was obtained as a colorless solid. Mp: $67.9^{\circ} \mathrm{C} .{ }^{1} \mathbf{H}$ NMR $\left(400 \mathrm{MHz}, \mathrm{CDCl}_{3}\right) \delta 10.76$ (brs, $\left.1 \mathrm{H}, \mathrm{NHOCH}_{3}\right), 7.57$ (m, 1H, $\mathrm{Ar} H$ ), 7.50 (ddd, $J=9.4,2.6,1.6 \mathrm{~Hz}, 1 \mathrm{H}, \mathrm{ArH}$ ), ${ }^{13}$ C NMR $\left(101 \mathrm{MHz}, \mathrm{CDCl}_{3}\right) \delta 165.0,162.4(\mathrm{~d}, J=247.6 \mathrm{~Hz}), 133.7(\mathrm{~d}, J=6.9 \mathrm{~Hz}), 130.1$ (d, $J=$ $7.8 \mathrm{~Hz}), 122.8,118.9$ (d, $J=21.2 \mathrm{~Hz}), 114.4$ (d, $J=23.3 \mathrm{~Hz}), 64.0$. IR 2979 (w), 2940 (w), 1652 (s), $1587(\mathrm{~s}), 1519(\mathrm{~m}), 1483(\mathrm{~m}), 1226$ (s), 1046 (m), 937 (m), 816 (s). Mp and IR significative values are in accordance with the data reported in literature. ${ }^{32}$

\section{3-Chloro-N-methoxybenzamide (60)}<smiles>CONC(=O)c1cccc(Cl)c1</smiles>

60

Starting from commercially available 3-chlorobenzoyl chloride (875 g, 5.00 mmol), 3-chloro-N-methoxybenzamide 60 (830 mg, $4.47 \mathrm{mmol}, 89 \%$ yield) was obtained as a white solid. ${ }^{1} \mathbf{H}$ NMR $\left(400 \mathrm{MHz}, \mathrm{CDCl}_{3}\right)$ : $\delta 9.90$ (br s, $\left.1 \mathrm{H}, \mathrm{NHOCH}_{3}\right)$, $7.75(\mathrm{~s}, 1 \mathrm{H}, \operatorname{Ar} H), 7.64$ (d, $J=7.8 \mathrm{~Hz}, 1 \mathrm{H}, \operatorname{Ar} H), 7.45(\mathrm{dd}, J=8.1,2.1 \mathrm{~Hz}, 1 \mathrm{H}$, $\operatorname{Ar} H), 7.32(\mathrm{t}, J=7.9 \mathrm{~Hz}, 1 \mathrm{H}, \mathrm{Ar} H), 3.83\left(\mathrm{~s}, 3 \mathrm{H}, \mathrm{NHOCH}_{3}\right) .{ }^{13} \mathbf{C} \mathbf{N M R}(101 \mathrm{MHz}$, $\left.\mathrm{CDCl}_{3}\right): \delta 165.1,134.7,133.4,132.0,129.9,127.5,125.3,64.4$. IR $\vee 3187(\mathrm{w})$, 3006 (w), 2937 (w), 1646 (s), 1572 (s), 1519 (m), 1471 (m), 1298 (m), 1162 (m), 1048 (m), 944 (m), $904(\mathrm{~m})$. NMR values are in accordance with the data reported in literature. ${ }^{33}$

[31] N. Guimond, S. I. Gorelsky, K. Fagnou, J. Am. Chem. Soc. 2011, 133, 6449-6457.

[32] V.P. Semenov, O.B. Ratner, K.A. Ogloblin, Zhurnal Organicheskoi Khimii 1979, 15, 1870-1873. Compound 59 is purchaseable via Adlab Chemicals Building Blocks and Aurora Building Blocks.

[33] H. Zhong, D. Yang, S. Wang, J. Huang, Chem. Commun. 2012, 48, 3236-3238. 


\section{N-Methoxybenzo[d][1,3]dioxole-5-carboxamide (61)}

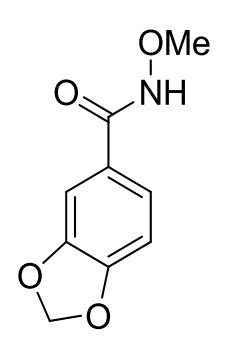

61

Starting from commercially available benzo[d][1,3]dioxole-5-carbonyl chloride (923 mg, $5.00 \mathrm{mmol}$ ), N-methoxybenzo[d][1,3]dioxole-5-carboxamide 61 (865 mg, $4.43 \mathrm{mmol}, 89 \%$ yield) was obtained as a white solid. ${ }^{1} \mathbf{H}$ NMR (400 MHz, $\left.\mathrm{CDCl}_{3}\right)$ $\delta 10.59$ (br s, $1 \mathrm{H}, \mathrm{NHOCH}_{3}$ ), 7.31 (d, $\left.J=8.2,1.8 \mathrm{~Hz}, 1 \mathrm{H}, \mathrm{ArH}\right), 7.23$ (s, 1H, $\mathrm{ArH}$ ), $6.69(\mathrm{~d}, J=8.1, \mathrm{~Hz}, 1 \mathrm{H}, \mathrm{ArH}), 5.92\left(\mathrm{~s}, 2 \mathrm{H}, \mathrm{OCH}_{2} \mathrm{O}\right), 3.75\left(\mathrm{~s}, 3 \mathrm{H}, \mathrm{NHOCH}_{3}\right) .{ }^{13} \mathrm{C}$ 63.9. IR $\vee 2982(\mathrm{w}), 2938(\mathrm{w}), 1650$ (m), 1605 (m), 1478 (s), 1438 (m), 1300 (m), 1252 (s), 1093 (m), 1034 (s), 927 (s), 839 (m). HRMS (ESI) calcd for $\mathrm{C}_{9} \mathrm{H}_{10} \mathrm{NO}_{4}{ }^{+}[\mathrm{M}+\mathrm{H}]^{+}$196.0604; found 196.0604.

\section{N-Methoxy-1-naphthamide (62)}<smiles>CONC(=O)c1cccc2ccccc12</smiles>

62

For this starting material the synthesis was performed on $1.00 \mathrm{mmol}$ scale

Starting from commercially available 1-naphthoyl chloride (191 mg, $1.00 \mathrm{mmol}$ ), N-methoxy-1-naphthamide 62 (183 mg, $0.909 \mathrm{mmol}, 91 \%$ yield) was obtained as a white solid. ${ }^{1} \mathbf{H}$ NMR $\left(300 \mathrm{MHz}, \mathrm{DMSO}-d_{6}\right) \delta 8.72$ (br s, $\left.1 \mathrm{H}, \mathrm{NHOCH}_{3}\right), 8.28$ (m, 1H, ArH), 8.10-7.90 (m, 2H, ArH), 7.71-7.41 (m, 4H, ArH), 3.94 (s, 3H, $\left.\mathrm{NHOCH}_{3}\right) .{ }^{13} \mathrm{C}$ NMR $\left(75 \mathrm{MHz}, \mathrm{CDCl}_{3}\right) \delta 167.3,133.6,131.4,130.3,129.6,128.3,127.4,126.6$, 125.6, 125.1, 124.5, 64.8. IR 2943 (w), 2907 (w), 2827 (w), 1631 (s), 1617 (s), 1591 (m), 1537 (w), $1318(w), 1262(w), 1063(\mathrm{~m}), 958(\mathrm{~m}), 889(\mathrm{w})$. NMR values are in accordance with the data reported in literature. ${ }^{34}$

[34] S. Rakshit, C. Grohmann, T. Besset, F. Glorius, J. Am. Chem. Soc. 2011, 133, 2350-2353. 


\subsection{Optimization of the Ru-Catalyzed Indolization of Arenes via C-H activation.}

GP5: In a vial, N-methoxybenzamide 14 (15.0 mg, $0.100 \mathrm{mmol}), 1$-(3-1-methyl- $1 H$-indole)- $1 H$ $1 \lambda_{3}$-benzo[b]iodo-3(2H)-one 9a (1.10 equiv.), $\left[\mathrm{RuCl}_{2} \text { (p-cymene) }\right]_{2} 67$ (2.45 mg, $4.00 \mu \mathrm{mol}, 4$ mol\%) and the relative additives $(16 \mathrm{~mol} \%)$ were dissolved in the specified dry solvent $(0.1 \mathrm{M})$ under nitrogen. the reaction mixture was degassed (freeze-thaw-pump) and stirred at the reported $\mathrm{T}$ in ${ }^{\circ} \mathrm{C}$ overnight. Then the reaction was stopped, the organic layer washed with a saturated solution of $\mathrm{NaHCO}_{3}(2 \mathrm{ml})$ and the solvent removed under reduced pressure. Flash column chromatography (Pentane:EtOAc 2:1) afforded the desired product 15a (see compound 15a's characterization for all the chemical data).

Table S9: Screening of solvents

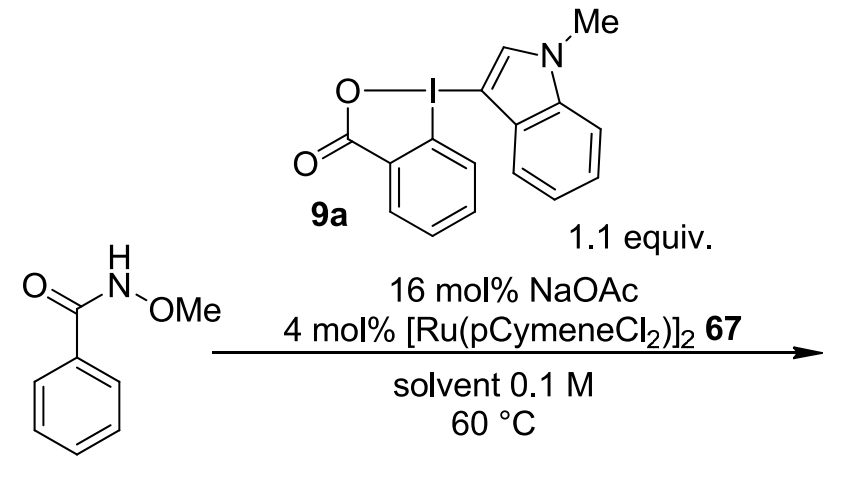

14

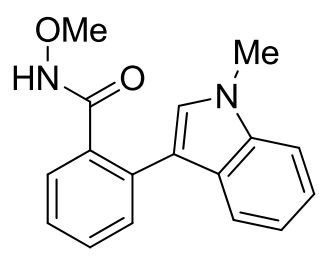

$15 a$

\begin{tabular}{ccc}
\hline Entry & Solvent & Yield\% $^{\mathbf{a}}$ \\
\hline 1 & DCM & - \\
2 & DCE & - \\
$\mathbf{3}$ & MeOH & $\mathbf{4 3 \%}$ \\
4 & EtOH & $34 \%$ \\
5 & $t$-BuOH & - \\
6 & t-AmylOH & - \\
7 & Toluene & - \\
8 & Xylene & - \\
9 & Dioxane & $28 \%$ \\
10 & MeCN & -
\end{tabular}




\begin{tabular}{|c|c|c|}
\hline 11 & DMF & - \\
\hline 12 & 2,2,2-Trifluoroethanol (TFE) & $55 \%$ \\
\hline 13 & Hexafluoroisopropanol (HFIP) & $30 \%$ \\
\hline 14 & $\begin{array}{c}\text { Nonafluoroisopropanol } \\
\text { (NFIP):HFIP 1:9 }\end{array}$ & - \\
\hline 15 & HFIP:DCE 9:1 & - \\
\hline 16 & HFIP:DCE 8:2 & $35 \%$ \\
\hline 17 & HFIP:DCE 1:1 & - \\
\hline 18 & Monofluoroethanol (MFE) & $21 \%$ \\
\hline
\end{tabular}

Table S10: Screening of the Temperature

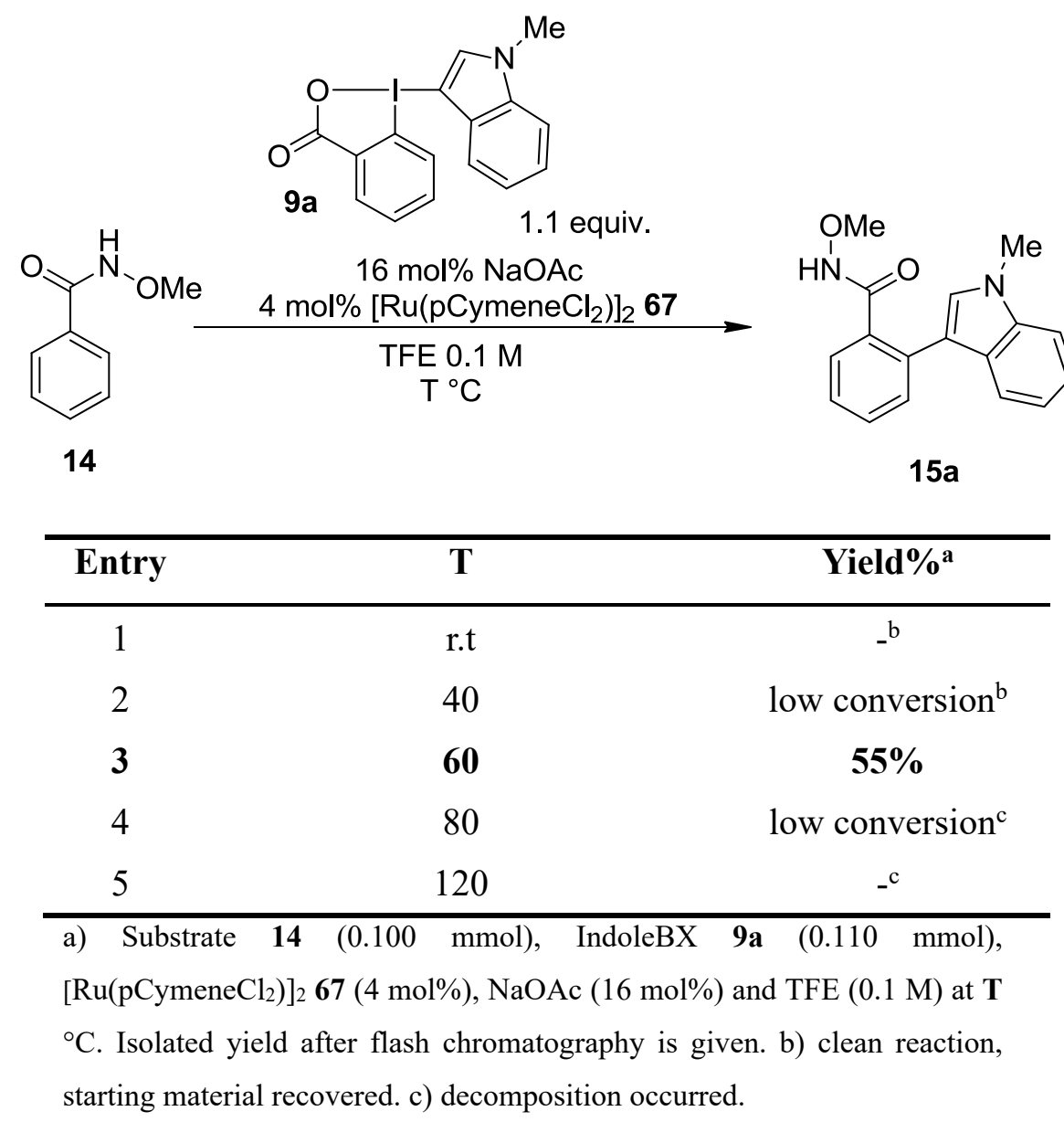


Table S11: Screening of the base

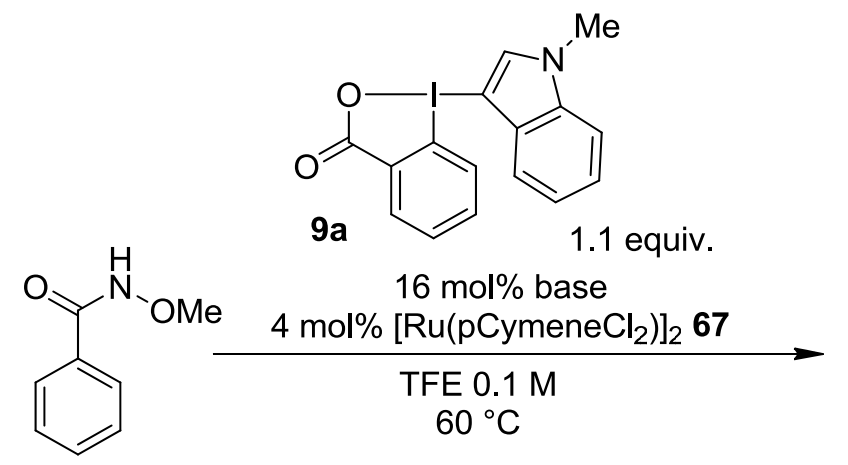

14

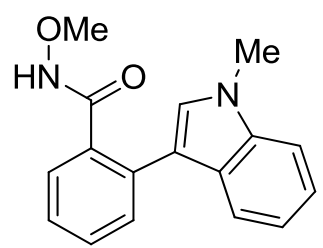

$15 a$

\begin{tabular}{|c|c|c|}
\hline Entry & Base & Yield $\%$ \\
\hline 1 & LiOPiv & - \\
\hline 2 & $\mathrm{Li}_{2} \mathrm{CO}_{3}$ & - \\
\hline 3 & LiMes & $-b$ \\
\hline 4 & $\mathrm{NaOAc}$ & $55 \%$ \\
\hline 5 & $\mathrm{Na}_{2} \mathrm{CO}_{3}$ & - \\
\hline 6 & NaOPiv & $50 \%$ \\
\hline 7 & NaMes & $34 \%$ \\
\hline 8 & 63 & $-b$ \\
\hline 9 & 64 & $68 \% \%^{c}$ \\
\hline 10 & 65 & $65 \%{ }^{b}$ \\
\hline 11 & 66 & $66 \%$ b \\
\hline 12 & KOAc & - \\
\hline 13 & KOPiv & - \\
\hline 14 & KMes & $26 \%$ b \\
\hline 15 & CsOPiv & - \\
\hline 16 & $\mathrm{Cs}_{2} \mathrm{CO}_{3}$ & - \\
\hline
\end{tabular}

a) Substrate $\mathbf{1 4} \quad\left(\begin{array}{llllll}0.100 & \mathrm{mmol}), & \text { IndoleBX } & \mathbf{9 a} & (0.110 & \mathrm{mmol}) \text {, }\end{array}\right.$ $\left.\left[\mathrm{Ru}(\mathrm{pCymeneCl})_{2}\right)\right]_{2} \mathbf{6 7}(4 \mathrm{~mol} \%)$, base $(16 \mathrm{~mol} \%)$ and TFE $(0.1 \mathrm{M})$ at 60 ${ }^{\circ} \mathrm{C}$. Isolated yield after flash chromatography is given. b) decomposition occurred. c)clean reaction, starting material recovered. 
<smiles>[NH3+]OC(=O)c1ccccc1</smiles>

63<smiles>NC(=O)C1c2ccccc2Oc2ccccc21</smiles>

65

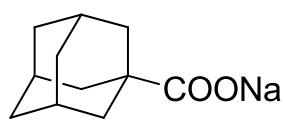

64<smiles>O=C(O[Na])C(c1ccccc1)c1ccccc1</smiles>

66

Table S12: Screening of the silver(I) additive

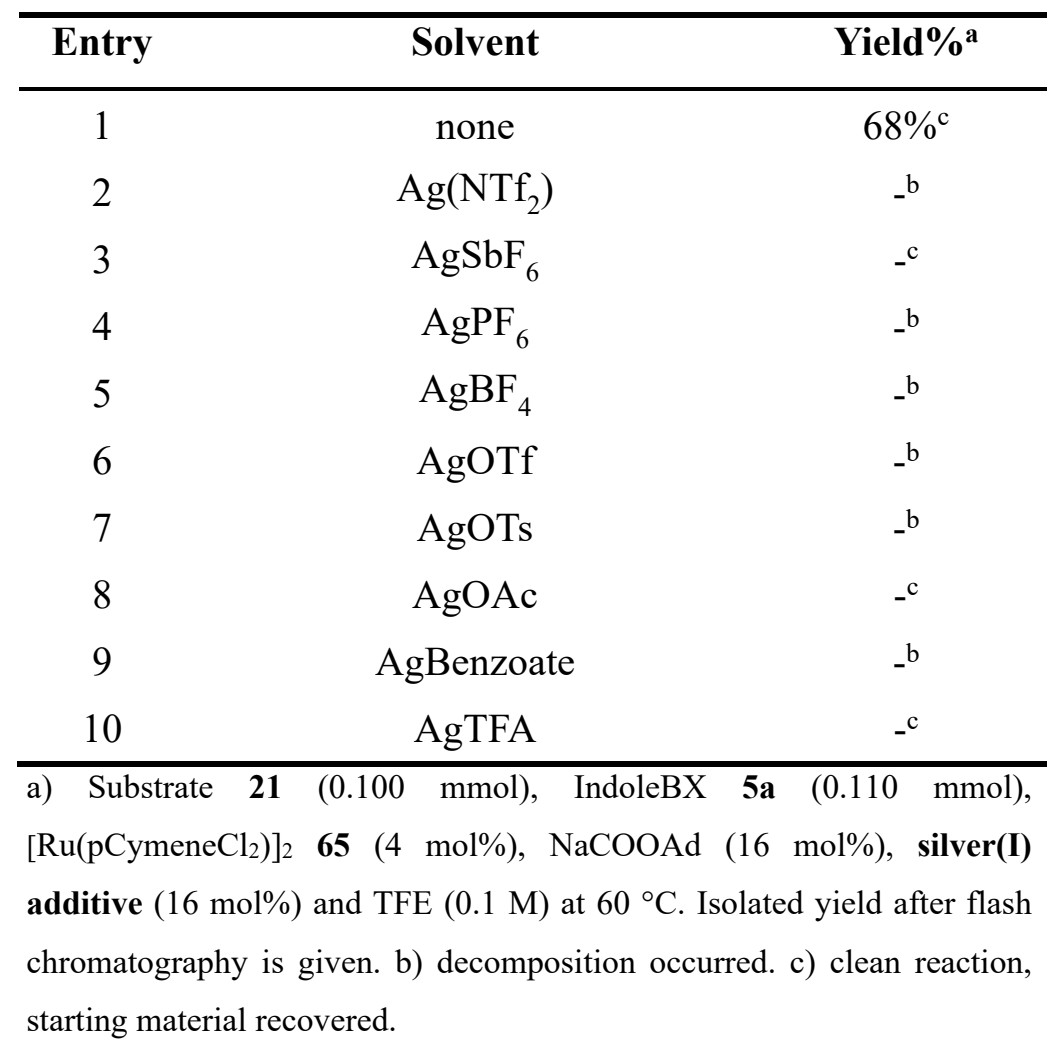




\subsection{Preparation of the Ruthenium Catalyst 68.}<smiles>Cc1cc(C(C)C)cc([R1](Cl)(Cl)C(Cl)(Cl)[R1](Cl)(Cl)c2cc(C)cc(C(C)C)c2)c1</smiles>

67

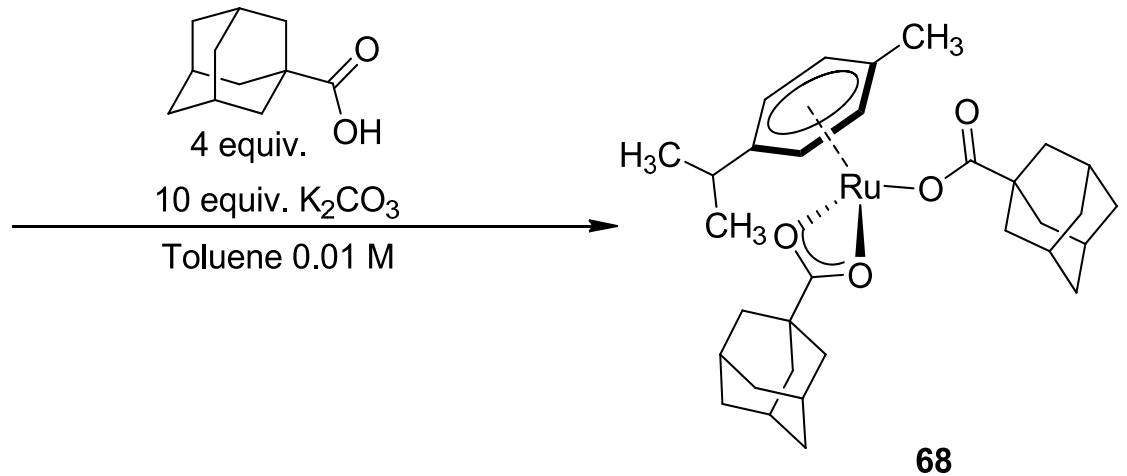

68

Following a reported procedure, ${ }^{35}\left[\mathrm{RuCl}_{2}(\mathrm{p} \text {-cymene })\right]_{2} 67$ (105 mg, $\left.0.163 \mathrm{mmol}\right)$, adamantane-1carboxylic acid (118 mg, $0.652 \mathrm{mmol}, 4.00$ equiv.) and $\mathrm{K}_{2} \mathrm{CO}_{3}$ (225 mg, $1.63 \mathrm{mmol}, 10.0$ equiv.) were suspended in toluene $(16.0 \mathrm{~mL}, 0.01 \mathrm{M})$ under $\mathrm{N}_{2}$. The resulting suspension was stirred for 3 hours at r.t.. The solvent was then removed in vacuo and the residue dissolved in dry $\mathrm{CH}_{2} \mathrm{Cl}_{2}(20$ $\mathrm{mL}$ ). The resulting suspension was filtered under $\mathrm{N}_{2}$ through a short plug of celite. The solvent was removed in vacuo to yield (catalyst) complex $68(90.0 \mathrm{mg}, 0.156 \mathrm{mmol}, 96 \%$ yield) as an orange solid. ${ }^{1} \mathbf{H}$ NMR $\left(300 \mathrm{MHz}, \mathrm{CDCl}_{3}\right): \delta 5.70(\mathrm{~d}, J=5.7 \mathrm{~Hz}, 2 \mathrm{H}, \mathrm{Ar} H), 5.49(\mathrm{~d}, J=5.7 \mathrm{~Hz}, 2 \mathrm{H}, \mathrm{Ar} H)$, 2.88 (hept, $\left.J=7.1 \mathrm{~Hz}, 1 \mathrm{H}, \operatorname{ArCH}\left(\mathrm{CH}_{3}\right)_{2}\right), 2.23$ (s, 3H, $\left.\mathrm{ArCH}\right), 1.92$ (t, $J=3.3 \mathrm{~Hz}, 6 \mathrm{H}$, $\left.\operatorname{ArCH}\left(\mathrm{CH}_{3}\right)_{2}\right), 1.78\left(\mathrm{~s}, 12 \mathrm{H}, \mathrm{AdCH} \mathrm{CH}_{2}\right), 1.63(\mathrm{~s}, 12 \mathrm{H}, \mathrm{AdCH}), 1.33$ (d, J=6.9 Hz, 6H, $\left.\mathrm{AdCH}\right) .{ }^{1} \mathrm{H}-$ NMR values are in accordance with the data reported in literature. ${ }^{35}$

[35] L. Ackermann, P. Novák, R. Vicente, N. Hofmann, Angew. Chem. Int. Ed. 2009, 48, 6045-6048. 


\subsection{Scope of the Ru-Catalyzed Indolization via C-H activation.}
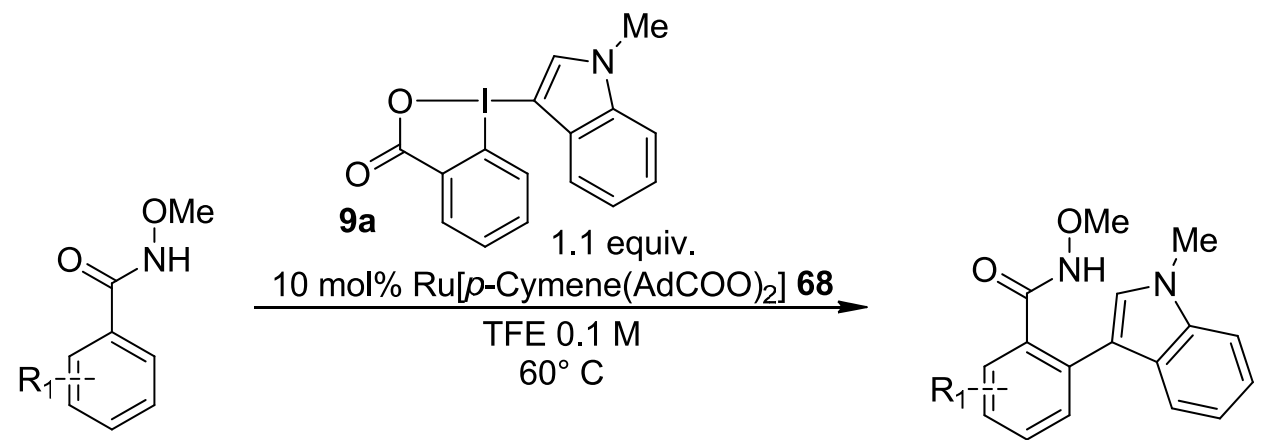

GP6: In a vial, freshly synthetized benzamide 14-62 $(50.0 \mathrm{mg}, 0.300 \mathrm{mmol}), 1-(3-1-$ methyl- $1 H$ indole)- $1 H-1 \lambda_{3}$-benzo[b]iodo-3(2H)-one $(0.330 \mathrm{mmol}, 1.10$ equiv. $)$ and freshly synthetized Ruthenium complex $68(18.2 \mathrm{mg}, 30.0 \mu \mathrm{mol}, 10 \mathrm{~mol} \%)$, were dissolved in dry TFE ( $3 \mathrm{ml}, 0.1 \mathrm{M})$ under nitrogen. the reaction mixture was degassed (freeze-thaw-pump) and stirred at $60{ }^{\circ} \mathrm{C}$ overnight. It was then allowed to cool down to r.t., washed with a saturated aqueous $\mathrm{NaHCO}_{3}(2 \mathrm{ml})$ and concentrated under reduced pressure. Flash column chromatography (Pentane:EtOAc) afforded the desired products 15a-15k. 
<smiles>CONC(=O)c1ccccc1-c1cn(C)c2ccccc12</smiles>

$15 a$

$68 \%$ yield<smiles>CONC(=O)c1ccc(Br)cc1-c1cn(C)c2ccccc12</smiles><smiles>CONC(=O)c1cc(Cl)ccc1-c1cn(C)c2ccccc12</smiles>

$15 i$

$75 \%$ yield
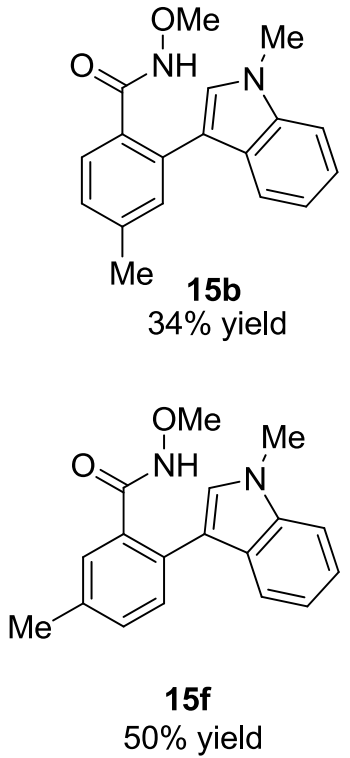
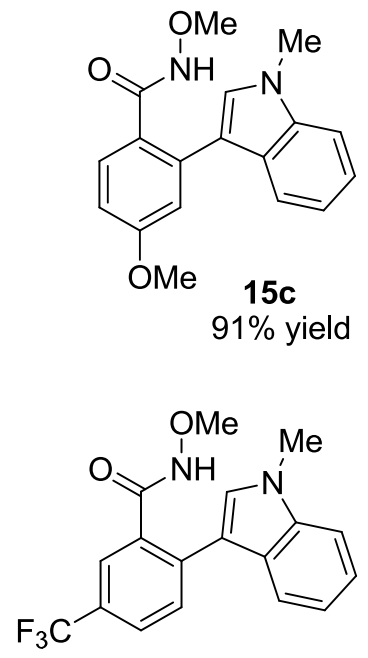

$15 \mathrm{~g}$ $60 \%$ yield

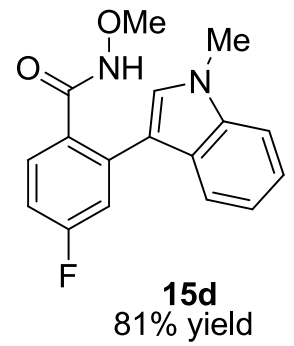<smiles>CONC(=O)c1cc(F)ccc1-c1cn(C)c2ccccc12</smiles>

$15 \mathrm{~h}$ $75 \%$ yield<smiles>CONC(=O)c1cc2c(cc1-c1cn(C)c3ccccc13)OCO2</smiles>

$80 \%$ yield

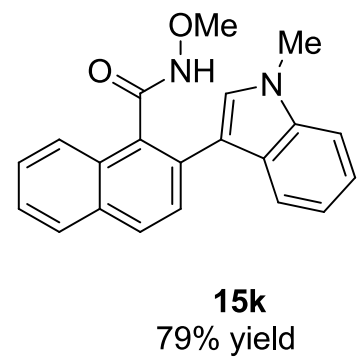

Figure S5: Scope with Methoxy-amides.

\section{N-Methoxy-2-(1-methyl-1H-indol-3-yl)benzamide (15a)}<smiles>COC(=O)c1ccccc1-c1cn(C)c2ccccc12</smiles>

$15 a$

Starting from N-methoxybenzamide 14 (45.3 mg, $0.300 \mathrm{mmol})$, N-methoxy-2(1-methyl-1H-indol-3-yl)benzamide 15a (57.0 mg, $0.203 \mathrm{mmol}, 68 \%$ yield) was obtained as a yellow oil. Rf: 0.4 (Pentane:EtOAc 2:1). ${ }^{1} \mathbf{H}$ NMR $(400 \mathrm{MHz}$, $\left.\mathrm{CDCl}_{3}\right) \delta 7.98\left(\right.$ br s, $\left.1 \mathrm{H}, \mathrm{N} H \mathrm{OCH}_{3}\right), 7.74(\mathrm{dd}, J=7.5,5.9 \mathrm{~Hz}, 1 \mathrm{H}, \mathrm{Ar} H), 7.67$ $(\mathrm{d}, J=7.9 \mathrm{~Hz}, 1 \mathrm{H}$, Indole $H$ ), 7.57 (dd, $J=7.8,1.4 \mathrm{~Hz}, 1 \mathrm{H}, \mathrm{Ar} H), 7.52$ (td, $J=$ 7.5, $1.4 \mathrm{~Hz}, 1 \mathrm{H}, \operatorname{Ar} H), 7.38$ (m, 2H, $\operatorname{Ar} H+$ Indole $H$ ), 7.30 (ddd, $J=8.2,6.9,1.1 \mathrm{~Hz}, 1 \mathrm{H}$, Indole $H$ ), 7.24 (s, $1 \mathrm{H}, \mathrm{CH}_{3} \mathrm{NCHC}$ ), 7.18 (ddd, $J=7.9,6.9,1.0 \mathrm{~Hz}, 1 \mathrm{H}$, Indole $\left.H\right), 3.85$ (s, 3H, NCH3), 3.46 $\left(\mathrm{s}, 3 \mathrm{H}, \mathrm{NHOCH}_{3}\right) .{ }^{13} \mathrm{C}$ NMR $\left(101 \mathrm{MHz}, \mathrm{CDCl}_{3}\right) \delta 168.4,137.0,132.5,132.4,130.8,130.7,129.5$, 128.1, 126.8, 126.7, 122.5, 120.3, 119.5, 113.5, 109.6, 63.9, 33.0. IR v 3209 (w), 3057 (w), 2968 
(w), 2934 (m), 2816 (w), 1655 (s), 1599 (w), 1547 (w), 1482 (s), 1464 (m), 1378 (m), 1329 (m), $1223(\mathrm{~m}), \quad 1161 \quad(\mathrm{w}), \quad 1034 \quad(\mathrm{~m}), 944 \quad(\mathrm{~m}), \quad 885 \quad(\mathrm{~m}), \quad$ HRMS $($ ESI) calcd for $\mathrm{C}_{17} \mathrm{H}_{16} \mathrm{~N}_{2} \mathrm{NaO}_{2}{ }^{+}[\mathrm{M}+\mathrm{Na}]^{+}$303.1104; found 303.1108

\section{N-Methoxy-4-methyl-2-(1-methyl-1H-indol-3-yl)benzamide (15b)}

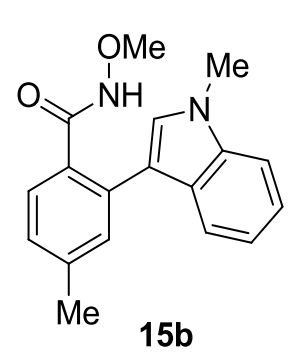

Starting from N-methoxy-4-methylbenzamide 53 (50.0 mg, $0.300 \mathrm{mmol})$, Nmethoxy-4-methyl-2-(1-methyl-1H-indol-3-yl)benzamide 15b (30.0 mg, 0.102 mmol, 34\% yield) was obtained as a yellow oil. Rf: 0.44 (Pentane:EtOAc 2:1). ${ }^{1} \mathbf{H}$ NMR $\left(400 \mathrm{MHz}, \mathrm{CDCl}_{3}\right) \delta 7.97$ (br s, $\left.1 \mathrm{H}, \mathrm{NHOCH}\right), 7.71-7.64(\mathrm{~m}, 2 \mathrm{H}$, $\operatorname{Ar} H), 7.40-7.34(\mathrm{~m}, 2 \mathrm{H}, \operatorname{Ar} H), 7.30(\mathrm{ddd}, J=8.2,6.9,1.2 \mathrm{~Hz}, 1 \mathrm{H}, \operatorname{Ar} H), 7.22$ - 7.16 (m, 3H, $\mathrm{ArH}), 3.85$ (s, 3H, NCH3), 3.44 (s, 3H, $\left.\mathrm{NHOCH}_{3}\right), 2.42$ (s, 3H, $\left.\mathrm{ArCH}_{3}\right) .{ }^{13} \mathrm{C}$ NMR $\left(101 \mathrm{MHz}, \mathrm{CDCl}_{3}\right) \delta 168.5,141.1,137.0,132.4,131.4,129.7,129.6,128.0$, 127.7, 126.8, 122.5, 120.4, 119.6, 113.8, 109.6, 63.8, 33.0, 21.5. IR $v 3200$ (w), 3047 (w), 2936 (w), 1662 (s), 1615 (m), 1543 (w), 1482 (m), $1370(\mathrm{w}), 1331$ (w), 1232 (w), $1160(\mathrm{w}), 1086(\mathrm{w})$, 1040 (w), 1015 (w), 912 (w), 887 (w), 834 (w). HRMS (ESI) calcd for $\mathrm{C}_{18} \mathrm{H}_{18} \mathrm{~N}_{2} \mathrm{NaO}_{2}{ }^{+}[\mathrm{M}+\mathrm{Na}]^{+}$ 317.1260 ; found 317.1264 .

\section{N,4-Dimethoxy-2-(1-methyl-1H-indol-3-yl)benzamide (15c)}

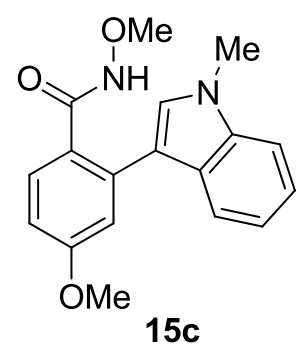

Starting from N,4-dimethoxybenzamide 54 (54.5 mg, $0.300 \mathrm{mmol}$ ), N,4dimethoxy-2-(1-methyl-1H-indol-3-yl)benzamide 15c (85.0 mg, $0.274 \mathrm{mmol}$, 91\% yield) was obtained as a yellow oil. Rf: 0.48 (Pentane:EtOAc 2:1). ${ }^{\mathbf{1}} \mathbf{H}$ NMR $\left(400 \mathrm{MHz}, \mathrm{CDCl}_{3}\right) \delta 8.02\left(\right.$ br s, $\left.1 \mathrm{H}, \mathrm{NHOCH}_{3}\right), 7.75(\mathrm{~d}, J=8.6 \mathrm{~Hz}, 1 \mathrm{H}$, $\operatorname{Ar} H), 7.66(\mathrm{dd}, J=8.0,1.0 \mathrm{~Hz}, 1 \mathrm{H}, \operatorname{Ar} H), 7.38(\mathrm{~d}, J=8.3 \mathrm{~Hz}, 1 \mathrm{H}, \operatorname{Ar} H), 7.30$ (ddd, $J=8.1,7.0,1.1 \mathrm{~Hz}, 1 \mathrm{H}, \operatorname{Ar} H), 7.22$ (s, 1H, NCHC), 7.18 (ddd, $J=8.1$, 7.0, 1.1 Hz, 1H, ArH), 7.03 (d, $J=2.6 \mathrm{~Hz}, 1 \mathrm{H}, \mathrm{Ar} H), 6.92(\mathrm{dd}, J=8.6,2.6 \mathrm{~Hz}, 1 \mathrm{H}, \mathrm{Ar} H), 3.85$ (s, $\left.3 \mathrm{H}, \mathrm{NCH}_{3}\right), 3.85\left(\mathrm{~s}, 3 \mathrm{H}, \mathrm{OCH}_{3}\right), 3.43\left(\mathrm{~s}, 3 \mathrm{H}, \mathrm{NHOCH}_{3}\right) .{ }^{13} \mathbf{C ~ N M R}\left(101 \mathrm{MHz}, \mathrm{CDCl}_{3}\right) \delta 168.1$, 161.3, 137.0, 134.3, 131.5, 128.0, 126.7, 124.8, 122.5, 120.4, 119.6, 115.7, 113.7, 112.5, 109.7, 63.8, 55.4, 33.0. IR v $3203(\mathrm{w}), 2961(\mathrm{w}), 2936(\mathrm{w}), 2838(\mathrm{w}), 1661(\mathrm{~s}), 1603(\mathrm{~s}), 1467$ (m), 1330 
(m), 1279 (m), 1238 (m), 1214 (m), 1084 (m), 1030 (m), 886 (w). HRMS (ESI) calcd for $\mathrm{C}_{18} \mathrm{H}_{18} \mathrm{~N}_{2} \mathrm{NaO}_{3}{ }^{+}[\mathrm{M}+\mathrm{Na}]^{+} 333.1210$; found 333.1210.

\section{5-Fluoro-N-methoxy-2-(1-methyl-1H-indol-3-yl)benzamide (15d)}

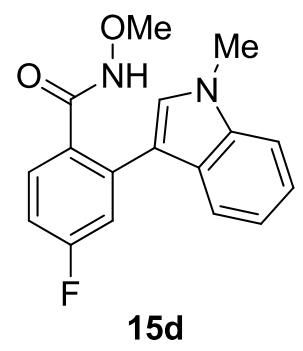

Starting from 4-fluoro-N-methoxybenzamide 55 (50.7 mg, $0.300 \mathrm{mmol})$, 4bromo-N-methoxy-2-(1-methyl-1H-indol-3-yl)benzamide $\quad \mathbf{1 5 d} \quad(78.0 \quad \mathrm{mg}$, $0.243 \mathrm{mmol}, 81 \%$ yield) was obtained as a yellow oil. Rf: 0.40 (Pentane:EtOAc 2:1). ${ }^{1} \mathbf{H}$ NMR $\left(400 \mathrm{MHz}, \mathrm{CDCl}_{3}\right) \delta 8.03\left(\mathrm{~s}, 1 \mathrm{H}, \mathrm{NHOCH}_{3}\right), 7.55(\mathrm{~d}, J=7.7$ $\mathrm{Hz}, 1 \mathrm{H}, \operatorname{Ar} H), 7.48(\mathrm{~m}, 1 \mathrm{H}, \operatorname{Ar} H), 7.42-7.35$ (m, 2H, ArH), 7.33 - 7.27 (m, 2H, ArH), 7.23 (s, 1H, NCHC), 7.17 (ddd, $J=8.0,7.0,1.1 \mathrm{~Hz}, 1 \mathrm{H}, \operatorname{Ar} H), 3.84$ $\left(\mathrm{s}, 3 \mathrm{H}, \mathrm{NCH}_{3}\right), 3.31\left(\mathrm{~s}, 3 \mathrm{H}, \mathrm{NHOCH}_{3}\right) .{ }^{13} \mathrm{C} \mathrm{NMR}\left(101 \mathrm{MHz}, \mathrm{CDCl}_{3}\right.$; two doublet were not resolved $) \delta 166.6,160.1(\mathrm{~d}, J=247.4 \mathrm{~Hz}), 136.8,135.2,129.2,128.6(\mathrm{~d}, J=8.5 \mathrm{~Hz}), 127.1,125.0$, 122.5, 120.4, $119.9(\mathrm{~d}, J=2.2 \mathrm{~Hz}), 118.01(\mathrm{~d}, J=23.3 \mathrm{~Hz}), 109.6,105.9,63.6,33.1 .{ }^{19} \mathbf{F}$ NMR (376 MHz, $\left.\mathrm{CDCl}_{3}\right) \delta$-111.7. IR v $3201(\mathrm{w}), 3055$ (w), 2984 (w), 2938 (w), 1932 (w), 1663 (m), 1551 (w), 1481 (m), 1455 (m), 1374 (m), 1330 (m), 1266 (s), 1245 (m), 1224 (m), 1161 (w), 1052 (m), 943 (w), 829 (s). HRMS (ESI) calcd for $\mathrm{C}_{17} \mathrm{H}_{15} \mathrm{FN}_{2} \mathrm{NaO}_{2}^{+}[\mathrm{M}+\mathrm{Na}]^{+}$321.1010; found 321.1008 .

\section{4-Bromo-N-methoxy-2-(1-methyl-1H-indol-3-yl)benzamide (15e)}

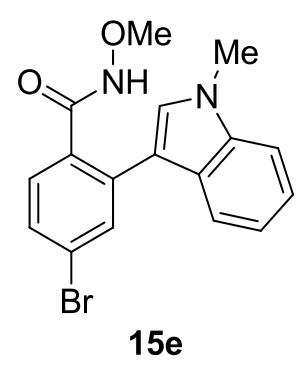

Starting from 4-bromo-N-methoxybenzamide 56 (69.0 mg, $0.300 \mathrm{mmol})$, 4bromo-N-methoxy-2-(1-methyl-1H-indol-3-yl)benzamide 15e (91.0 mg, 0.253 mmol, 84\% yield) was obtained as an orange oil. Rf: 0.35 (Pentane:EtOAc 2:1). ${ }^{1} \mathbf{H}$ NMR $\left(400 \mathrm{MHz}, \mathrm{CDCl}_{3}\right) \delta 8.04$ (br s, $\left.1 \mathrm{H}, \mathrm{NHOCH}_{3}\right), 7.75(\mathrm{~m}, 1 \mathrm{H}, \mathrm{ArH})$, $7.67(\mathrm{~d}, J=8.0 \mathrm{~Hz}, 1 \mathrm{H}, \operatorname{Ar} H), 7.39(\mathrm{~d}, J=8.2 \mathrm{~Hz}, 1 \mathrm{H}, \operatorname{Ar} H), 7.33(\mathrm{~m}, 1 \mathrm{H}$, $\operatorname{ArH}), 7.28$ - $7.24(\mathrm{~m}, 2 \mathrm{H}, \operatorname{Ar} H), 7.21(\mathrm{~m}, 1 \mathrm{H}, \operatorname{Ar} H), 7.07(\mathrm{td}, J=8.3,2.6 \mathrm{~Hz}$,

$1 \mathrm{H}, \mathrm{ArH}), 3.85\left(\mathrm{~s}, 3 \mathrm{H}, \mathrm{NCH}_{3}\right), 3.46\left(\mathrm{~s}, 3 \mathrm{H}, \mathrm{NHOCH}_{3}\right) .{ }^{13} \mathrm{C} \mathrm{NMR}\left(101 \mathrm{MHz}, \mathrm{CDCl}_{3}\right) \delta$ 167.4, 137.0, 134.6, 133.3, 131.2, 129.8, 128.4, 126.4, 125.2, 122.8, 120.7, 119.3, 112.3, 109.8, 63.9, 33.1 (one Carbon signal not resolved). IR $v 3186$ (w), 3063 (w), 2934 (w), 1656 (s), 1605 (s), 1580 (m), 1480 (s), 1364 (w), 1331 (m), 1265 (s), 1195 (m), 1082 (w), 1037 (m), 986 (w), 939 (w), 887 (m), 826 (m). HRMS (ESI) calcd for $\mathrm{C}_{17} \mathrm{H}_{15}{ }^{79} \mathrm{BrN}_{2} \mathrm{NaO}_{2}{ }^{+}[\mathrm{M}+\mathrm{Na}]^{+}$381.0209; found 381.0204 . 


\section{N-Methoxy-5-methyl-2-(1-methyl-1H-indol-3-yl)benzamide (15f)}<smiles>CONC(=O)c1cc(C)ccc1-c1cn(C)c2ccccc12</smiles>

$15 f$

Starting from N-methoxy-3-methylbenzamide $57(50.0 \mathrm{mg}, 0.300 \mathrm{mmol})$, N-methoxy-5-methyl-2-(1-methyl-1H-indol-3-yl)benzamide $\mathbf{1 5 f}$ (44.0 mg, $0.149 \mathrm{mmol}, 50 \%$ yield) was obtained as a yellow oil. Rf: 0.40 (Pentane:EtOAc 2:1). ${ }^{1} \mathbf{H}$ NMR (400 MHz, $\left.\mathrm{CD}_{2} \mathrm{Cl}_{2}\right) \delta 8.27$ (s, $1 \mathrm{H}, \mathrm{Ar} H$ ), $8.24(\mathrm{~s}, 1 \mathrm{H}, \mathrm{NHOCH}), 8.13(\mathrm{dd}, J=8.1,1.9 \mathrm{~Hz}, 1 \mathrm{H}, \mathrm{Ar} H), 7.67$ (dd, $J=$ 9.9, 8.0 Hz, 2H, ArH), 7.41 (dt, $J=8.3,0.9 \mathrm{~Hz}, 1 \mathrm{H}, \operatorname{Ar} H$ ), 7.34 (s, 1H, ArH), 7.29 (ddd, $J=8.2$, 7.0, 1.1 Hz, 1H, ArH), 7.18 (ddd, $J=8.0,7.0,1.1 \mathrm{~Hz}, 1 \mathrm{H}, \mathrm{ArH}), 3.92$ (s, 3H, NCH3), 3.85 (s, 3H, $\left.\mathrm{CH}_{3}\right), 3.49$ (s, 3H, $\left.\mathrm{NHOCH}_{3}\right) .{ }^{13} \mathbf{C}$ NMR $\left(101 \mathrm{MHz}, \mathrm{CD}_{2} \mathrm{Cl}_{2}\right) \delta 166.7,138.2,137.8,133.1,131.8$, 131.2, 131.0, 129.5, 128.6, 127.0, 123.1, 121.0, 119.9, 113.3, 110.4, 64.3, 52.7, 33.6. IR v 3181 (w), 2932 (w), 1713 (s), 1660 (s), 1606 (m), 1538 (w), 1466 (w), 1437 (w), 1289 (m), 1262 (s), 1248 (s), 1162 (w), 1128 (m), 1107 (m), 1038 (w). HRMS (ESI) calcd for $\mathrm{C}_{18} \mathrm{H}_{18} \mathrm{~N}_{2} \mathrm{NaO}_{2}{ }^{+}$ $[\mathrm{M}+\mathrm{Na}]^{+}$317.1260; found 317.1265 .

\section{N-Methoxy-2-(1-methyl-1H-indol-3-yl)-5-(trifluoromethyl)benzamide (15g)}<smiles>CONC(=O)c1cc(C(F)(F)F)ccc1-c1cn(C)c2ccccc12</smiles>

$15 \mathrm{~g}$

Starting from N-methoxy-3-(trifluoromethyl)benzamide $58(65.7 \mathrm{mg}$, $0.300 \quad$ mmol), N-methoxy-2-(1-methyl-1H-indol-3-yl)-5(trifluoromethyl)benzamide $15 \mathrm{~g}$ (63.0 $\mathrm{mg}, 0.181 \mathrm{mmol}, 60 \%$ yield) was obtained as a colorless oil. Rf: 0.37 (Pentane:EtOAc 2:1). ${ }^{1} \mathbf{H}$ NMR (400 $\left.\mathrm{MHz}, \mathrm{CDCl}_{3}\right) \delta 8.00$ (br s, $\left.1 \mathrm{H}, \mathrm{NHOCH}_{3}\right), 7.91(\mathrm{~s}, 1 \mathrm{H}, \mathrm{ArH}), 7.70-7.62$ (m, 2H, ArH), 7.59 (d, $J=8.0 \mathrm{~Hz}, 1 \mathrm{H}, \operatorname{Ar} H), 7.33$ (m, $1 \mathrm{H}, \operatorname{Ar} H), 7.27$ (m, 1H, $\mathrm{ArH}$ ), 7.24 (s, 1H, NCHC), 7.14 (ddd, $J=8.0,6.9,1.1 \mathrm{~Hz}, 1 \mathrm{H}, \mathrm{ArH}), 3.79$ (s, 3H, NCH3), 3.45 $\left(\mathrm{s}, 3 \mathrm{H}, \mathrm{NHOCH}_{3}\right) .{ }^{13} \mathbf{C} \mathbf{N M R}\left(101 \mathrm{MHz}, \mathrm{CDCl}_{3}\right) \delta 167.1,137.1,136.4,132.6,130.9,128.8,128.7$, $127.3,126.7,126.4,123.80$ (q, $\left.J=272.1 \mathrm{~Hz}, C F_{3}\right), 122.8,120.8,119.2,112.2,109.9,64.1,33.1$. ${ }^{19}$ F NMR $\left(376 \mathrm{MHz}, \mathrm{CDCl}_{3}\right) \delta$-62.5. IR $v 3187(\mathrm{w}), 2978(\mathrm{w}), 2935$ (w), 1657 (m), $1617(\mathrm{w})$, 1549 (w), 1469 (w), 1331 (s), 1274 (w), 1173 (m), 1159 (m), 1127 (s), 1092 (m), 947 (w), 911 (w), 849 (w). HRMS (ESI) calcd for $\mathrm{C}_{18} \mathrm{H}_{15} \mathrm{~F}_{3} \mathrm{~N}_{2} \mathrm{NaO}_{2}{ }^{+}[\mathrm{M}+\mathrm{Na}]^{+}$371.0978; found 371.0979. 


\section{5-Fluoro-N-methoxy-2-(1-methyl-1H-indol-3-yl)benzamide (15h)}<smiles>CONC(=O)c1cc(F)ccc1-c1cn(C)c2ccccc12</smiles>

$(\mathrm{dd}, J=8.2,2.0 \mathrm{~Hz}, 1 \mathrm{H}, \operatorname{Ar} H), 7.40(\mathrm{~m}, 1 \mathrm{H}, \operatorname{Ar} H), 7.32(\mathrm{dd}, J=7.0,1.2 \mathrm{~Hz}, 1 \mathrm{H}, \operatorname{Ar} H), 7.25(\mathrm{~s}$, 1H, NCHC), 7.21 (ddd, $J=8.0,6.9,1.1 \mathrm{~Hz}, 1 \mathrm{H}, \mathrm{ArH}), 3.85$ (s, 3H, $\left.\mathrm{NCH}_{3}\right), 3.45$ (s, 3H, $\mathrm{NHOCH}_{3}$ ). ${ }^{13} \mathbf{C}$ NMR $\left(101 \mathrm{MHz}, \mathrm{CDCl}_{3}\right) \delta 167.6,163.8(\mathrm{~d}, J=250.9 \mathrm{~Hz}), 137.0,135.4,135.3(\mathrm{~d}, J=9.4 \mathrm{~Hz})$, $131.8(\mathrm{~d}, J=9.5 \mathrm{~Hz}), 128.4,128.3,126.4,122.7,120.7,117.10(\mathrm{~d}, J=21.5 \mathrm{~Hz}), 113.79(\mathrm{~d}, J=$ 21.9 Hz), 112.5, 109.8, 63.9, 33.1. ${ }^{19} \mathbf{F}$ NMR (376 MHz, $\left.\mathrm{CDCl}_{3}\right) \delta$-109.3. IR v $3179(\mathrm{w}), 3055$ (w), 2932 (m), 2853 (w), 1662 (s), 1586 (m), 1550 (w), 1479 (s), 1329 (w), 1256 (w), 1222 (w), 1163 (w), 1089 (m), 1038 (m), 956 (w), 911 (m), 882 (m), 824 (w). HRMS (ESI) calcd for $\mathrm{C}_{17} \mathrm{H}_{15} \mathrm{FN}_{2} \mathrm{NaO}_{2}^{+}[\mathrm{M}+\mathrm{Na}]^{+}$321.1010; found 321.1011.

\section{5-Chloro-N-methoxy-2-(1-methyl-1H-indol-3-yl)benzamide (15i)}<smiles>CONC(=O)c1cc(Cl)ccc1-c1cn(C)c2ccccc12</smiles>

Starting from 3-chloro-N-methoxybenzamide $60(55.7 \mathrm{mg}, 0.300 \mathrm{mmol})$, N-methoxy-2-(1-methyl-1H-indol-3-yl)-5-(trifluoromethyl)benzamide 15i (70.9 mg, $0.225 \mathrm{mmol}, 75 \%$ yield) was obtained as a yellow oil. Rf: 0.35 (Pentane:EtOAc 2:1). ${ }^{1} \mathbf{H}$ NMR $\left(400 \mathrm{MHz}, \mathrm{CDCl}_{3}\right) \delta 7.81$ (br s, $1 \mathrm{H}$, $\left.\mathrm{NHOCH}_{3}\right), 7.64(\mathrm{dd}, J=8.0,1.3 \mathrm{~Hz}, 1 \mathrm{H}, \mathrm{Ar} H), 7.60(\mathrm{~d}, J=7.7 \mathrm{~Hz}, 1 \mathrm{H}$, $\operatorname{Ar} H), 7.45-7.37(\mathrm{~m}, 2 \mathrm{H}, \operatorname{Ar} H), 7.35(\mathrm{~d}, J=7.9 \mathrm{~Hz}, 1 \mathrm{H}, \operatorname{Ar} H), 7.29(\mathrm{~m}, 1 \mathrm{H}, \operatorname{Ar} H), 7.19(\mathrm{~s}, 1 \mathrm{H}$, $\mathrm{NCHC}), 7.15(\mathrm{t}, J=7.5 \mathrm{~Hz}, 1 \mathrm{H}, \mathrm{ArH}), 3.87\left(\mathrm{~s}, 3 \mathrm{H}, \mathrm{NCH}_{3}\right), 3.15(\mathrm{~s}, 3 \mathrm{H}, \mathrm{NHOCH}) .{ }^{13} \mathbf{C ~ N M R}(101$ $\left.\mathrm{MHz}, \mathrm{CDCl}_{3}\right) \delta 166.9,137.0,133.6,132.7,132.0,131.1,130.9,129.5,128.2,126.6,122.7,120.6$, 119.3, 112.3, 109.8, 64.0, 33.1. IR $v 3185$ (w), 3058 (w), 2931 (m), 2852 (w), 1656 (s), $1543(\mathrm{w})$, 1482 (m), 1466 (m), 1374 (w), 1330 (w), 1256 (w), 1163 (w), 1100 (m), 1041 (w), 943 (m), 824 (w). HRMS (ESI) calcd for $\mathrm{C}_{17} \mathrm{H}_{15} \mathrm{ClN}_{2} \mathrm{NaO}_{2}{ }^{+}[\mathrm{M}+\mathrm{Na}]^{+}$337.0714; found 337.0720. 


\section{N-Methoxy-6-(1-methyl-1H-indol-3-yl)benzo[d][1,3]dioxole-5-carboxamide (15j)}

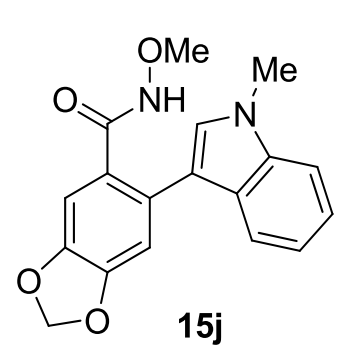

Starting from N-methoxybenzo[d][1,3]dioxole-5-carboxamide 61 (58.6 mg, $0.300 \mathrm{mmol}), \mathrm{N}$-methoxy-6-(1-methyl-1H-indol-3-yl)benzo[d][1,3]dioxole5-carboxamide $\mathbf{1 5 j}$ (78.0 $\mathrm{mg}, 0.240 \mathrm{mmol}, 80 \%$ yield) was obtained as a white oil. Rf: 0.48 (Pentane:EtOAc 2:1). ${ }^{1} \mathbf{H}$ NMR (400 MHz, $\left.\mathrm{CD}_{2} \mathrm{Cl}_{2}\right) \delta$ $7.49(\mathrm{~d}, J=8.0 \mathrm{~Hz}, 1 \mathrm{H}, \mathrm{Ar} H), 7.40(\mathrm{~d}, J=8.3 \mathrm{~Hz}, 1 \mathrm{H}$, Indole $H), 7.33-7.25$ (m, 3H, Indole $H$ ), 7.14 (ddd, $J=8.0,6.9,1.0 \mathrm{~Hz}, 1 \mathrm{H}, \operatorname{Ar} H), 6.85$ (d, $J=8.1$ $\mathrm{Hz}, 1 \mathrm{H}$, Indole $H$ ), 6.01 (s, 2H, $\mathrm{OCH}_{2} \mathrm{O}$ ), 3.84 (s, 3H, NCH$), 3.35$ (s, 3H, NHOCH$)_{3}$. (NHOMe proton present at $8.2 \mathrm{ppm}){ }^{13} \mathbf{C}$ NMR $\left(101 \mathrm{MHz}, \mathrm{CD}_{2} \mathrm{Cl}_{2}\right.$; the signals of two aromatic carbons were not resolved) $\delta 166.8,149.5,146.1,137.0,129.4,127.1,126.9,124.1,122.4,120.2,115.2,109.8$, 107.0, 106.8, 101.7, 63.6, 33.1. IR $v 3191(\mathrm{w}), 2967(\mathrm{w}), 2934(\mathrm{w}), 2899(\mathrm{w}), 1656(\mathrm{~m}), 1628(\mathrm{~m})$, 1480 (m), 1448 (s), 1374 (w), 1340 (m), 1250 (s), 1224 (w), 1132 (w), 1040 (s), 1017 (w), 929 (m), 833 (w). HRMS (ESI) calcd for $\mathrm{C}_{18} \mathrm{H}_{16} \mathrm{~N}_{2} \mathrm{NaO}_{4}{ }^{+}[\mathrm{M}+\mathrm{Na}]^{+}$347.1002; found 347.1002.

\section{N-Methoxy-2-(1-methyl-1H-indol-3-yl)-1-naphthamide (15k)}

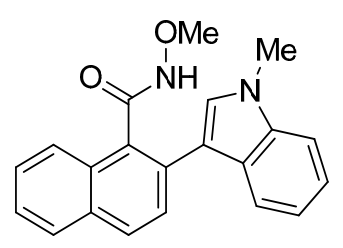

$15 \mathrm{k}$

Starting from N-methoxy-1-naphthamide $62(60.4 \mathrm{mg}, 0.300 \mathrm{mmol}), \mathrm{N}-$ methoxy-2-(1-methyl-1H-indol-3-yl)-1-naphthamide 15k (78.0 mg, 0.236 mmol, 79\% yield) was obtained as a colorless oil. Rf: 0.35 (Pentane:EtOAc 2:1). ${ }^{1} \mathbf{H}$ NMR (400 MHz, $\left.\mathrm{CDCl}_{3}\right) \delta 8.06(\mathrm{~d}, J=8.5 \mathrm{~Hz}, 1 \mathrm{H}, \mathrm{Ar} H), 8.00-$ $7.95\left(\mathrm{~m}, 2 \mathrm{H}, \mathrm{ArH}+\mathrm{NHOCH}_{3}\right), 7.89(\mathrm{~m}, 1 \mathrm{H}, \mathrm{ArH}), 7.80(\mathrm{t}, J=8.6 \mathrm{~Hz}, 2 \mathrm{H}$, $\operatorname{Ar} H$ ), 7.58 (ddd, $J=8.4,6.8,1.4 \mathrm{~Hz}, 1 \mathrm{H}, \operatorname{Ar} H), 7.52$ (ddd, $J=8.1,6.9,1.3 \mathrm{~Hz}, 1 \mathrm{H}, \operatorname{Ar} H), 7.41$ (d, $J=9.7 \mathrm{~Hz}, 2 \mathrm{H}, \mathrm{Ar} H), 7.32(\mathrm{~m}, 1 \mathrm{H}, \mathrm{ArH}), 7.22(\mathrm{~m}, 1 \mathrm{H}, \mathrm{ArH}), 3.86\left(\mathrm{~s}, 3 \mathrm{H}, \mathrm{NCH}_{3}\right), 3.66(\mathrm{~s}, 3 \mathrm{H}$, $\left.\mathrm{NHOCH}_{3}\right) .{ }^{13} \mathrm{C}$ NMR $\left(101 \mathrm{MHz}, \mathrm{CDCl}_{3}\right) \delta 168.3,137.1,131.8,131.3,131.1,130.0,128.9,128.5$, 128.0, 127.6, 127.4, 126.9, 126.0, 124.8, 122.3, 120.2, 119.4, 113.1, 109.8, 64.0, 33.0. IR v 3186 (w), $3056(\mathrm{w}), 2958(\mathrm{w}), 2929(\mathrm{~m}), 2854(\mathrm{w}), 1651$ (s), 1615 (m), 1545 (w), $1479(\mathrm{~m}), 1384(\mathrm{w})$, 1339 (w), 1264 (w), 1230 (w), 1134 (w), 1101 (w), 1074 (m), 1019 (m), 892 (w), 821 (s). HRMS (ESI) calcd for $\mathrm{C}_{21} \mathrm{H}_{18} \mathrm{~N}_{2} \mathrm{NaO}_{2}{ }^{+}[\mathrm{M}+\mathrm{Na}]^{+}$353.1260; found 353.1256. 


\section{Crystal Structure and DSC Measurements.}

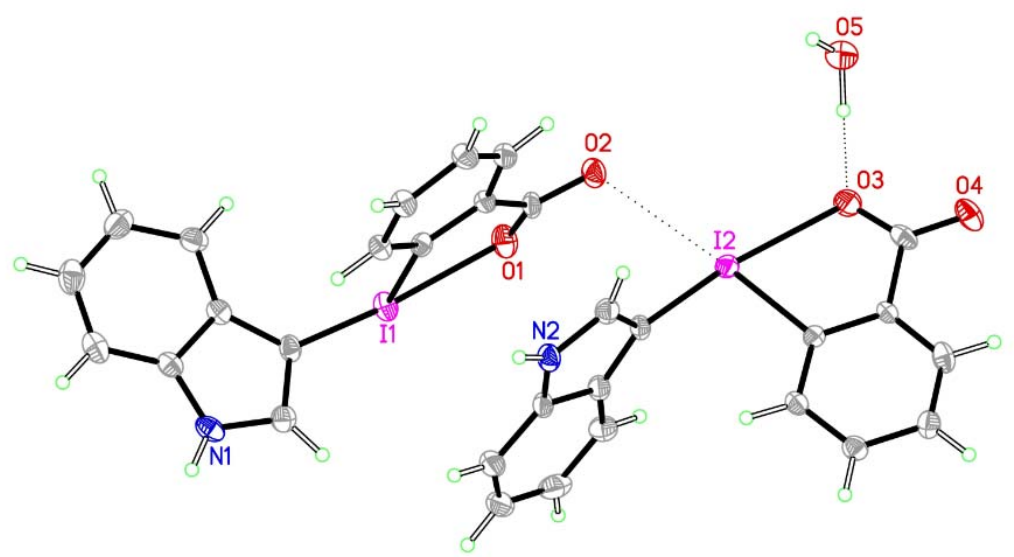

A single crystal was grown by slow diffusion of the solution of $\mathbf{9 b}$ in $\mathrm{MeOD} / \mathrm{CCl}_{4}$ mixture. Supplementary crystallographic data for this compound have been deposited at Cambridge Crystallographic Data Centre (1540821) and can be obtained free of charge via www.ccdc.cam.ac.uk/data_request/cif.

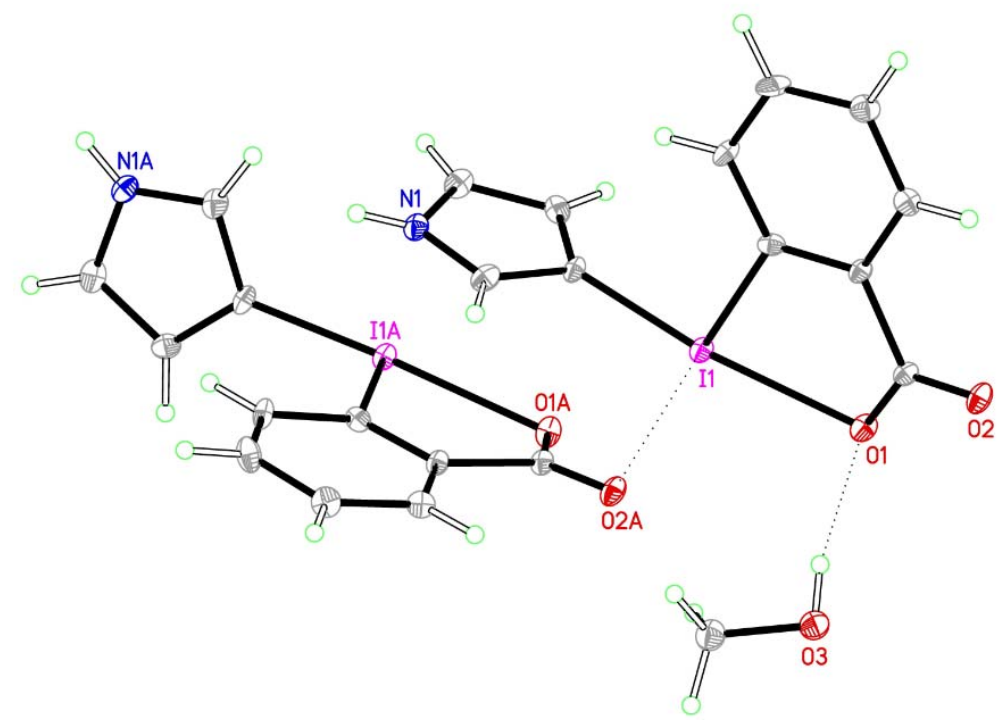

A single crystal was grown by slow diffusion of the solution of $9 m$ in $\mathrm{MeOD} / \mathrm{CCl}_{4}$ mixture. Supplementary crystallographic data for this compound have been deposited at Cambridge Crystallographic Data Centre (1541174) and can be obtained free of charge via www.ccdc.cam.ac.uk/data_request/cif. 
DSC measurements of compound 9a

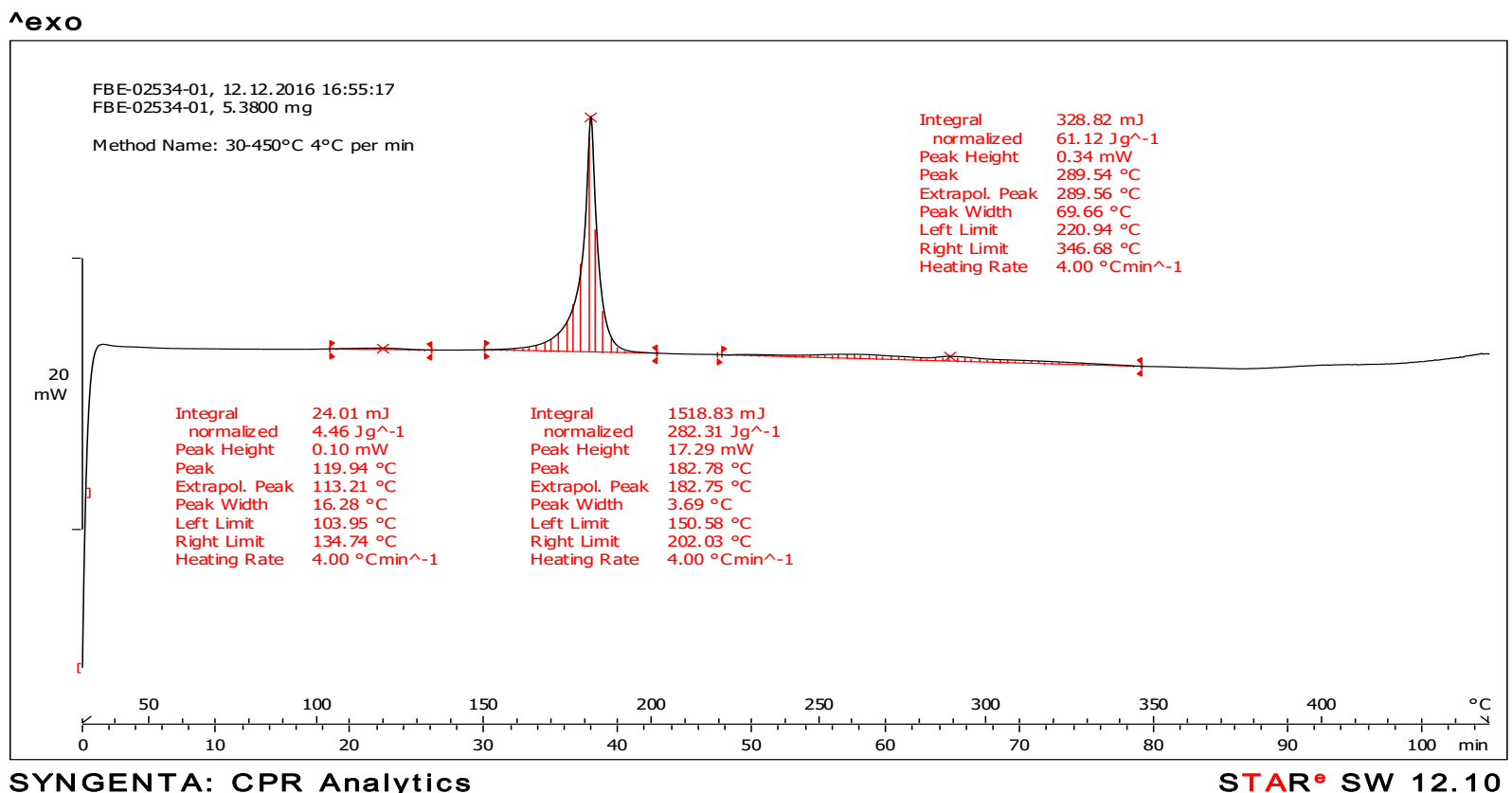

DSC measurements of compound 9n

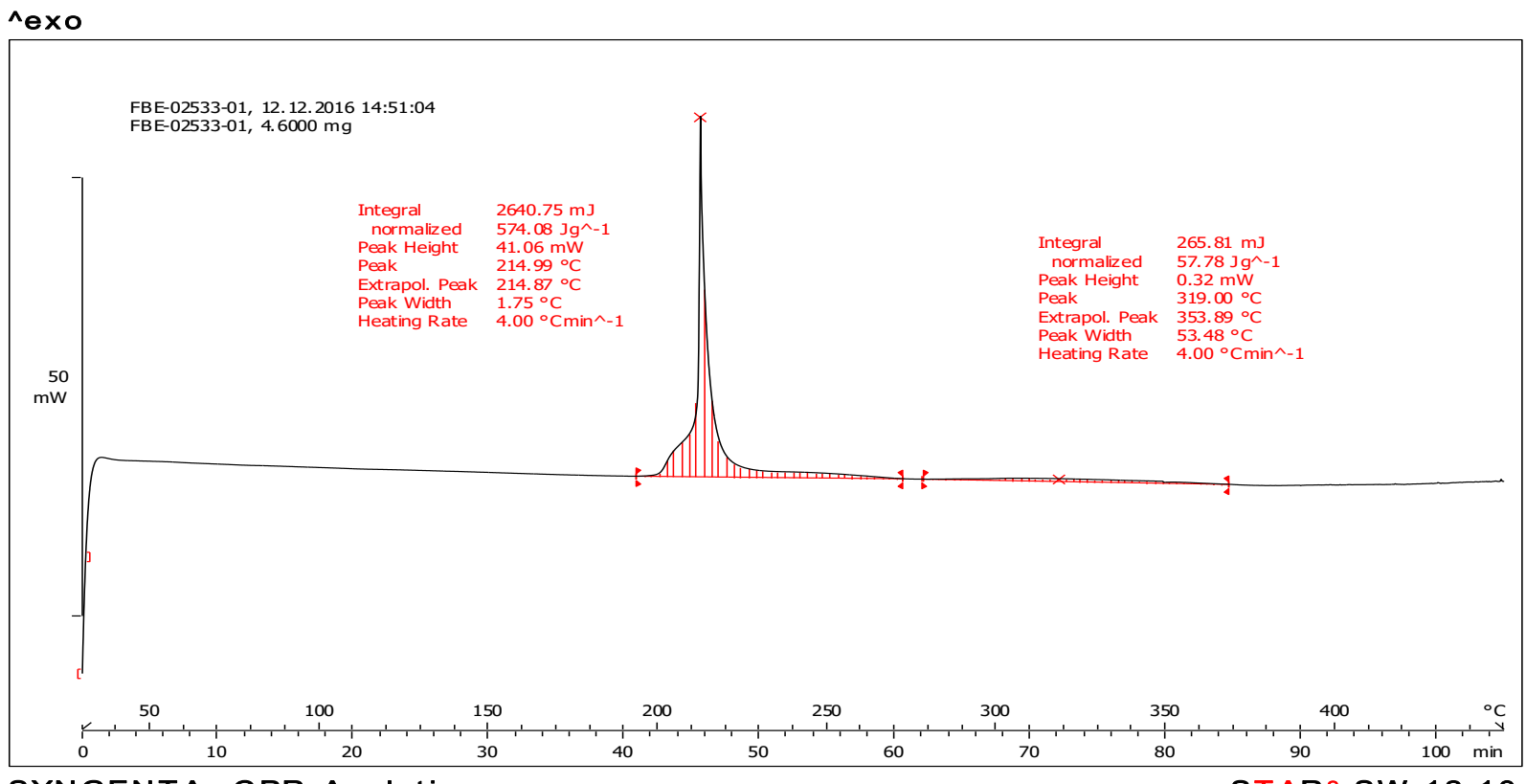

SYNGENTA: CPR Analytics

STAR $^{\mathrm{S}} \mathrm{SW} 12.10$ 
DSC measurements of compound 9o

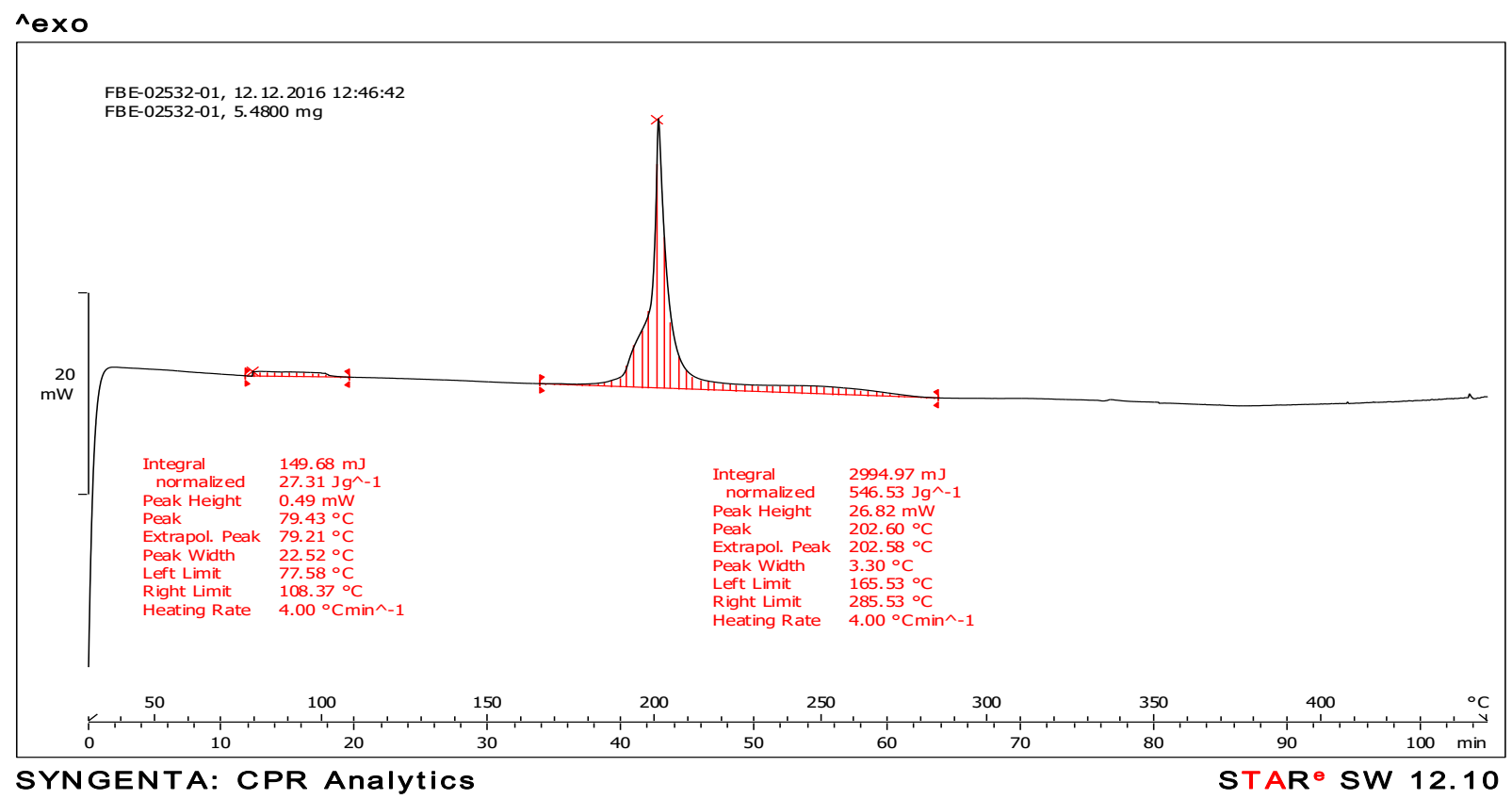


6. Spectra of new compounds 
1-(3-1-Methyl-1 $H$-indole)-1 $H$-1 $\lambda_{3}$-benzo[b]iodo-3(2H)-one (9a)

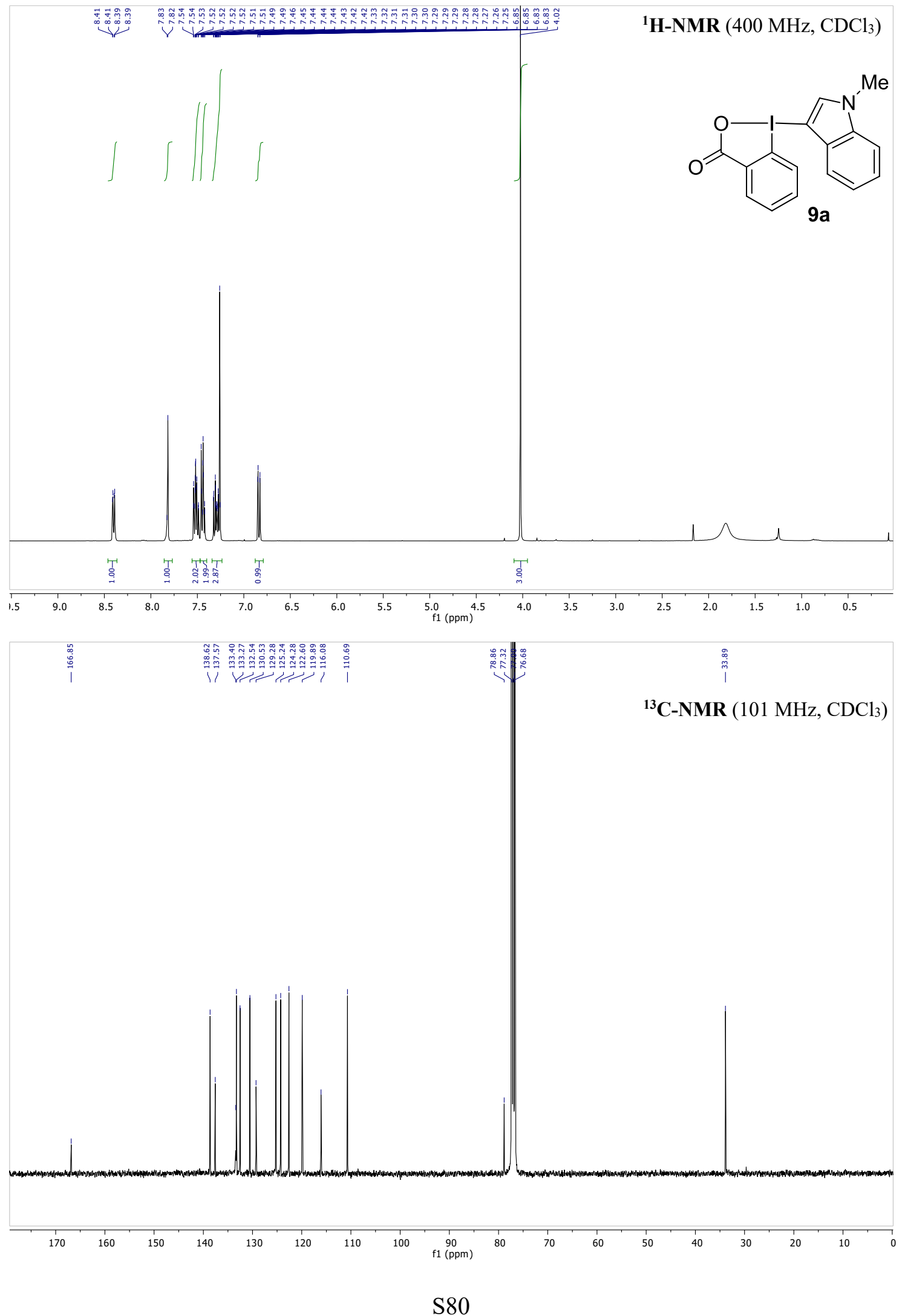


1-(3-1H-Indole)-1H-1 $\lambda_{3}$-benzo $[b]$ iodo-3(2H)-one (9b)

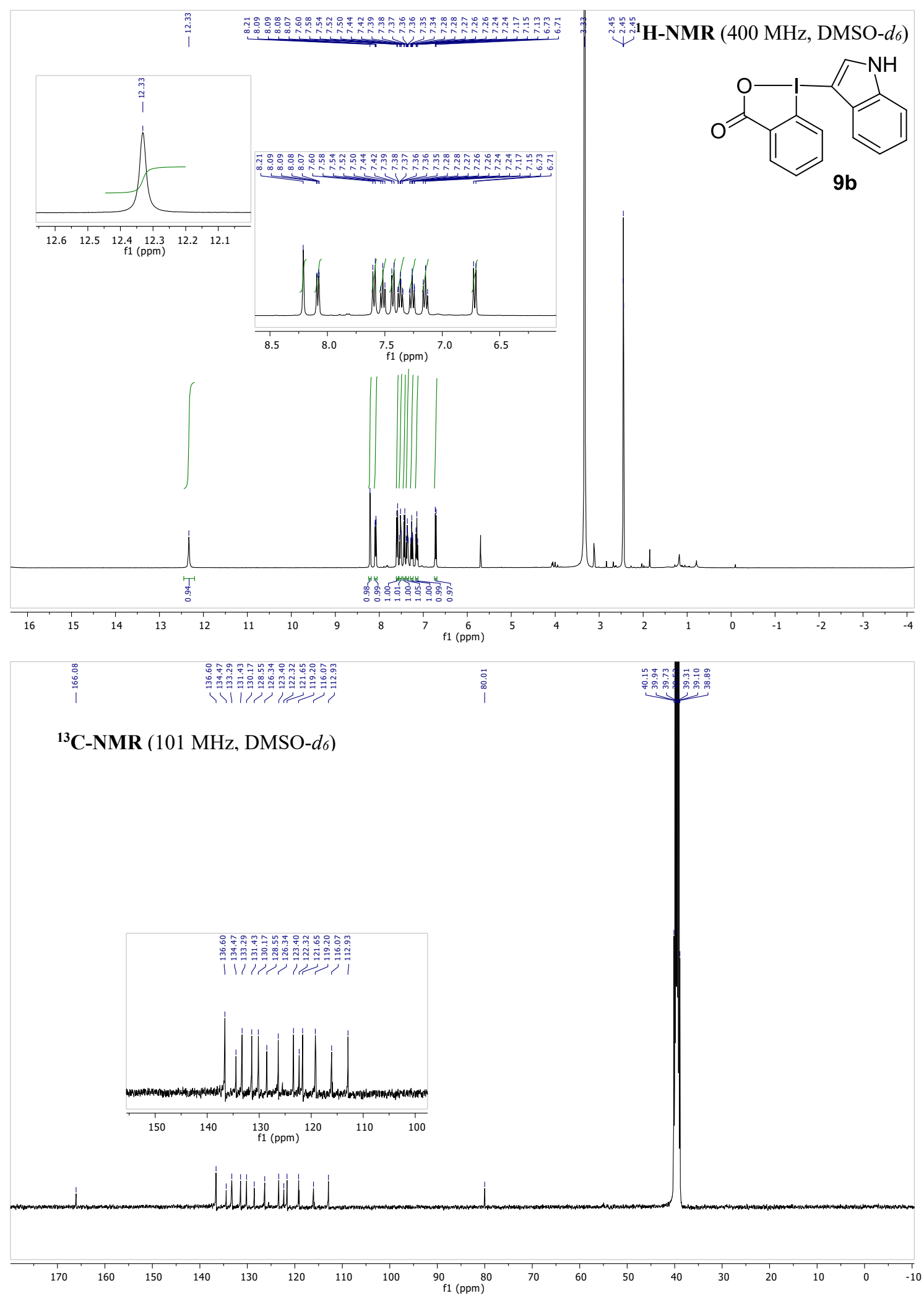


1-(3-1-(But-3-en-1-yl)-1H-indole)-1 $H$-1 $\lambda_{3}$-benzo[b]iodo-3(2H)-one (9c)

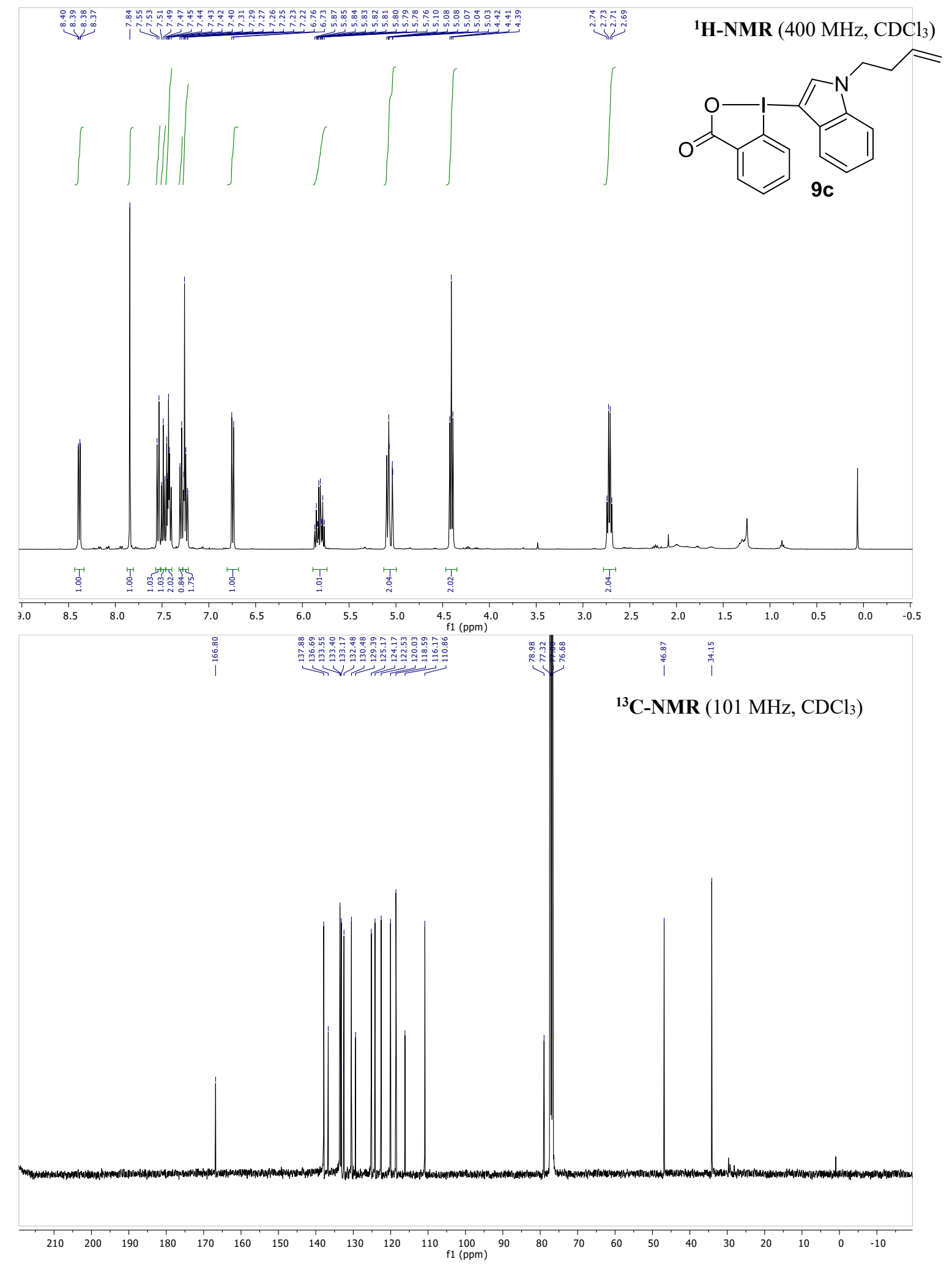


1-(3-1-(2-((Triisopropylsilyl)oxy)ethyl)-1H-indole)-1 $H$-1 $\lambda_{3}$-benzo[b]iodo-3(2H)-one (9d)
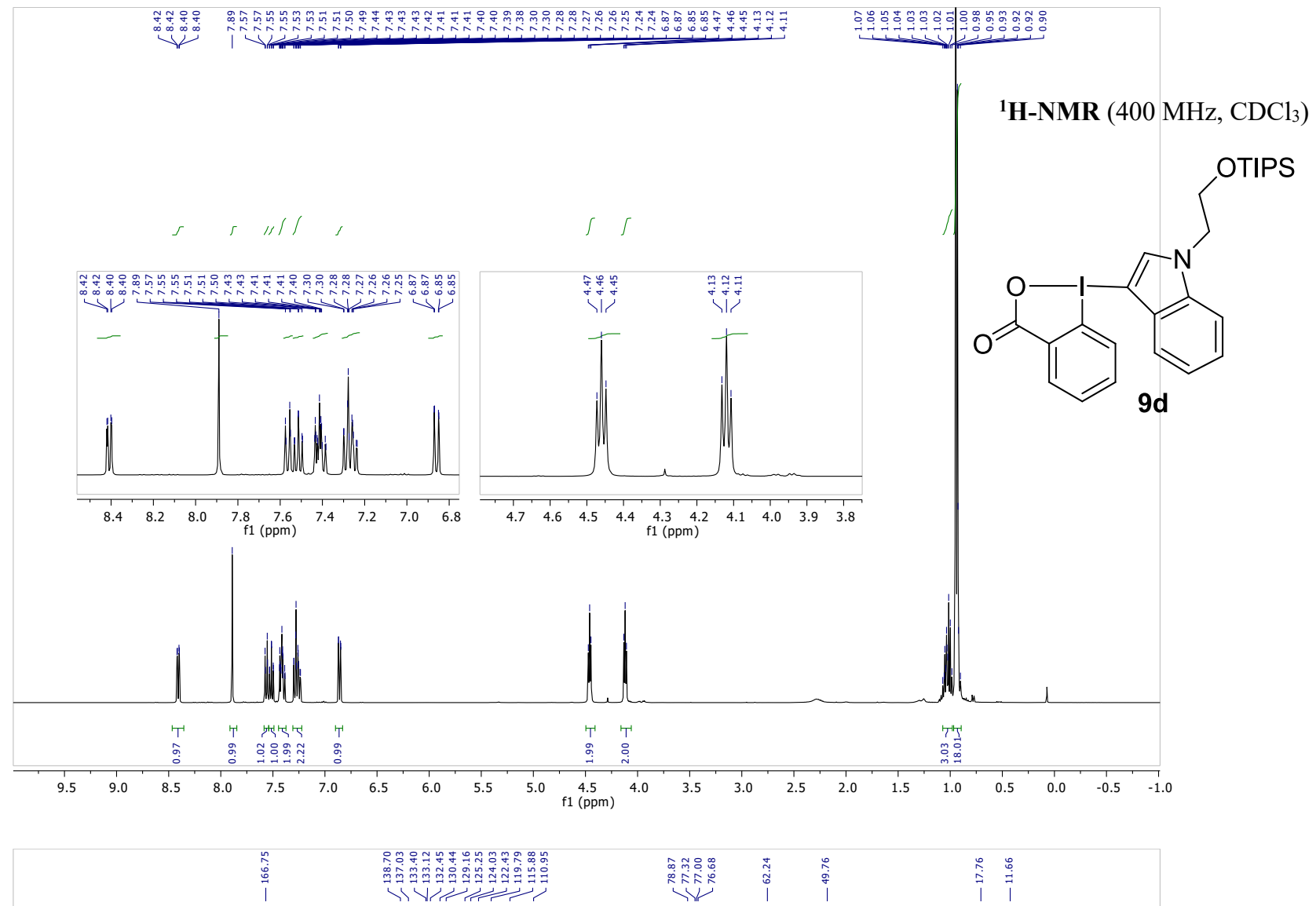

${ }^{13}$ C-NMR (101 MHz, $\left.\mathrm{CDCl}_{3}\right)$

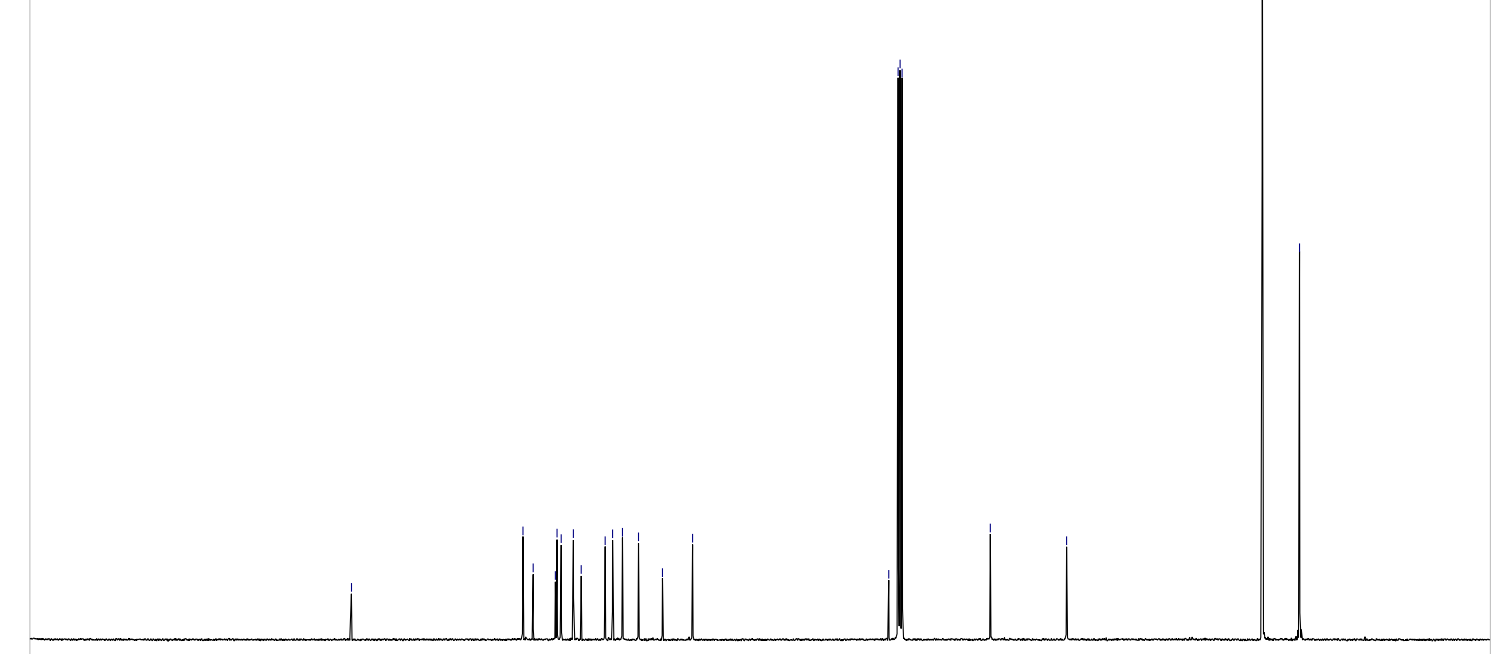

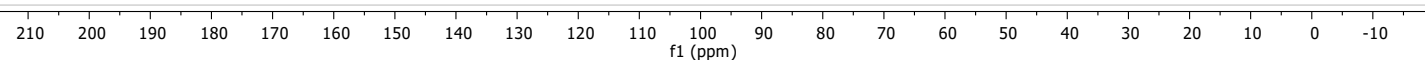




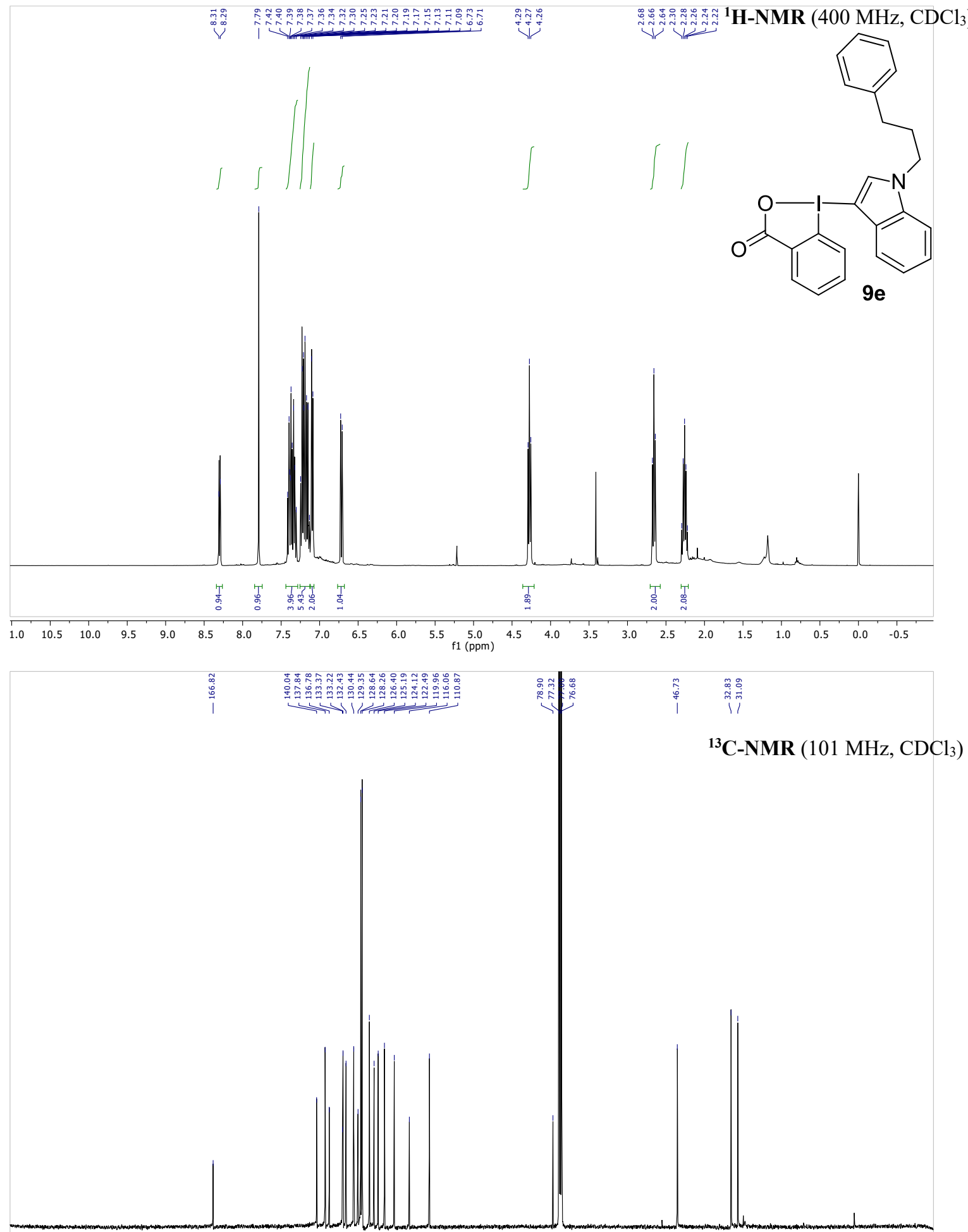

$\begin{array}{lllllllllllllllllllllll}210 & 200 & 190 & 180 & 170 & 160 & 150 & 140 & 130 & 120 & 110 & 100 & 90 & 80 & 70 & 60 & 50 & 40 & 30 & 20 & 10 & 0 & -10\end{array}$ 


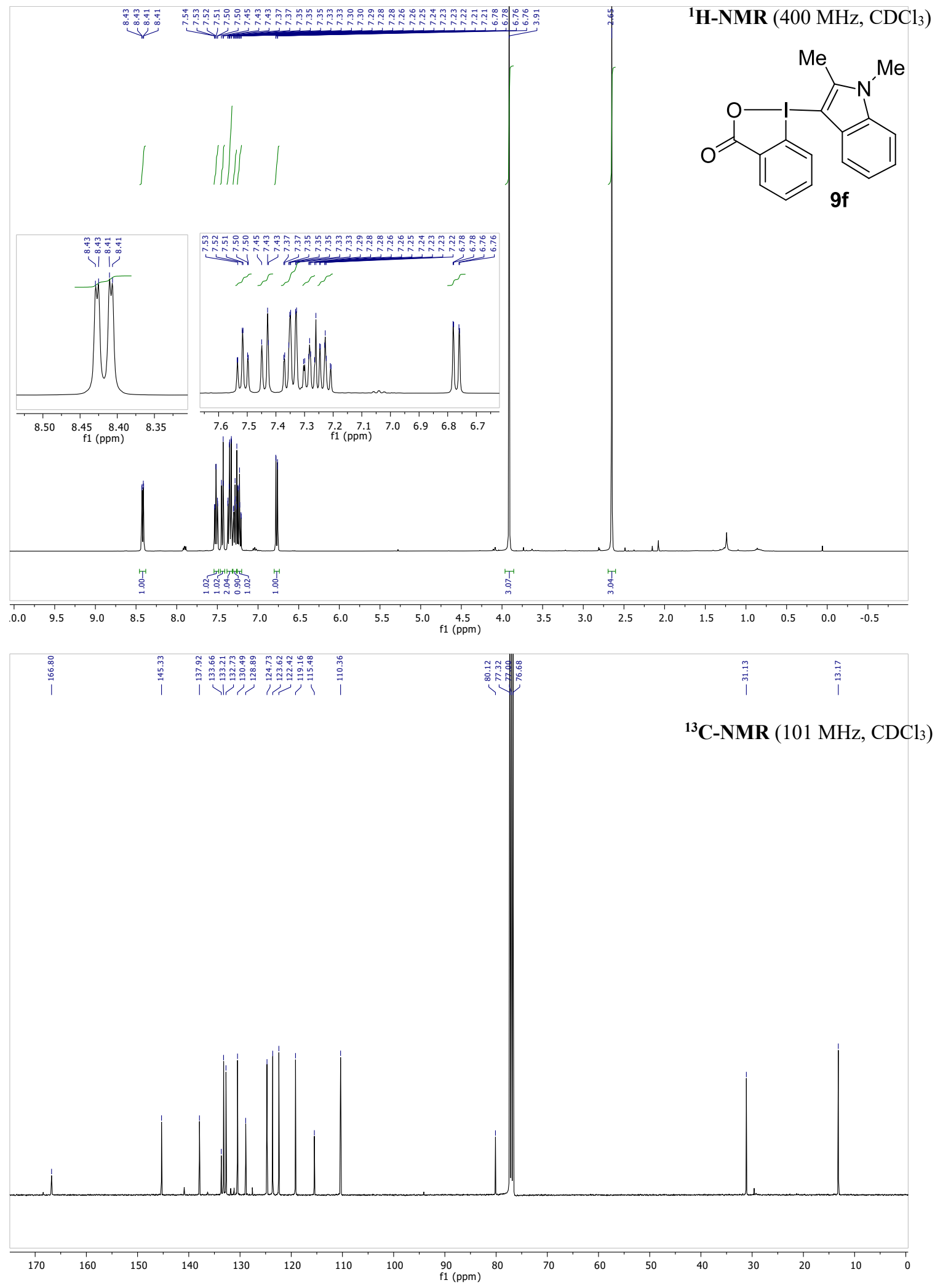


1-(3-5-Methoxy-1-methyl-1H-indole)-1H-1 $\lambda_{3}$-benzo[b]iodo-3(2H)-one (9g)
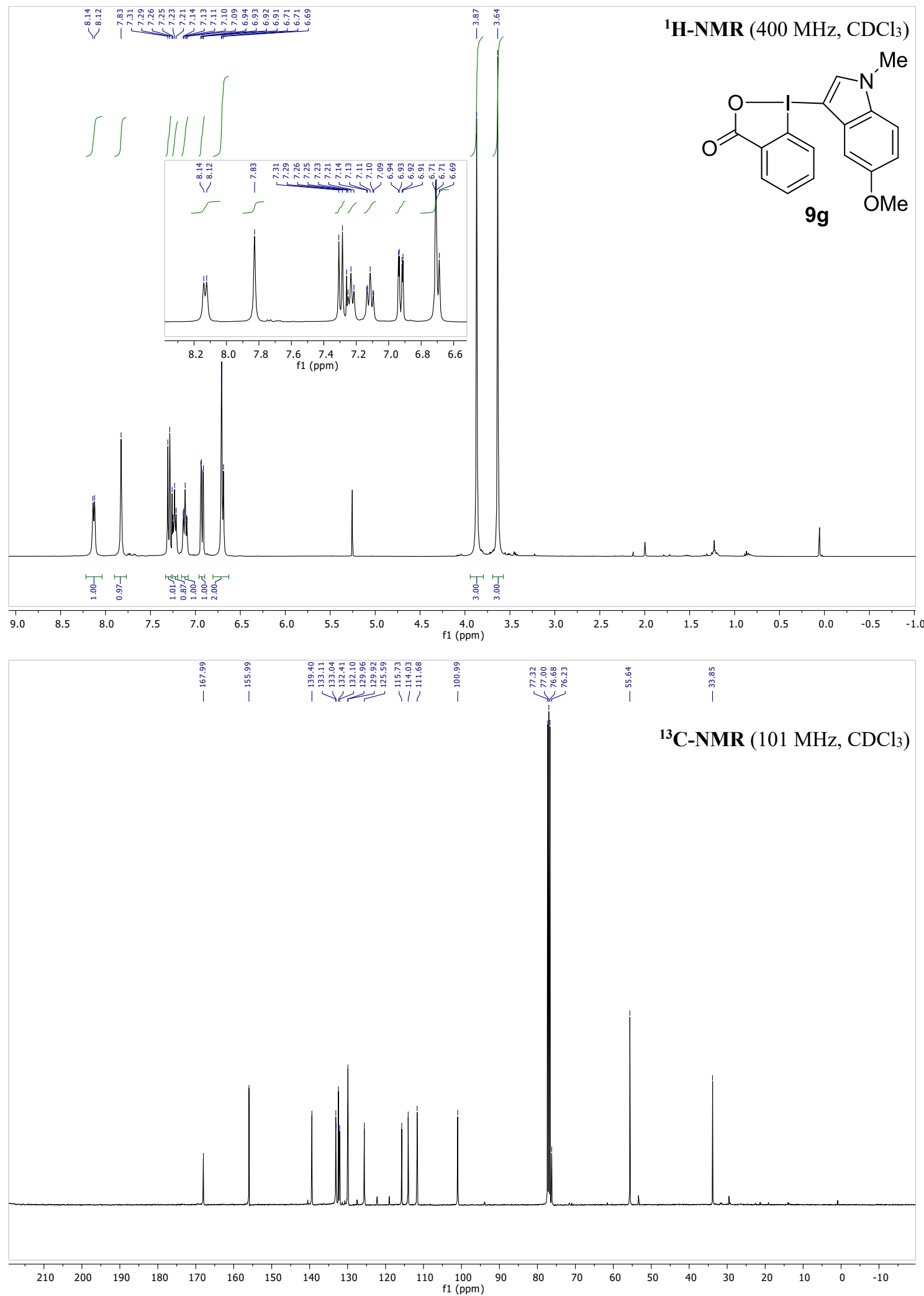
1-(3-5-fluoro-1-methyl-1H-indole)-1H-1 $\lambda_{3}$-benzo $[b]$ iodo-3(2H)-one (9h)

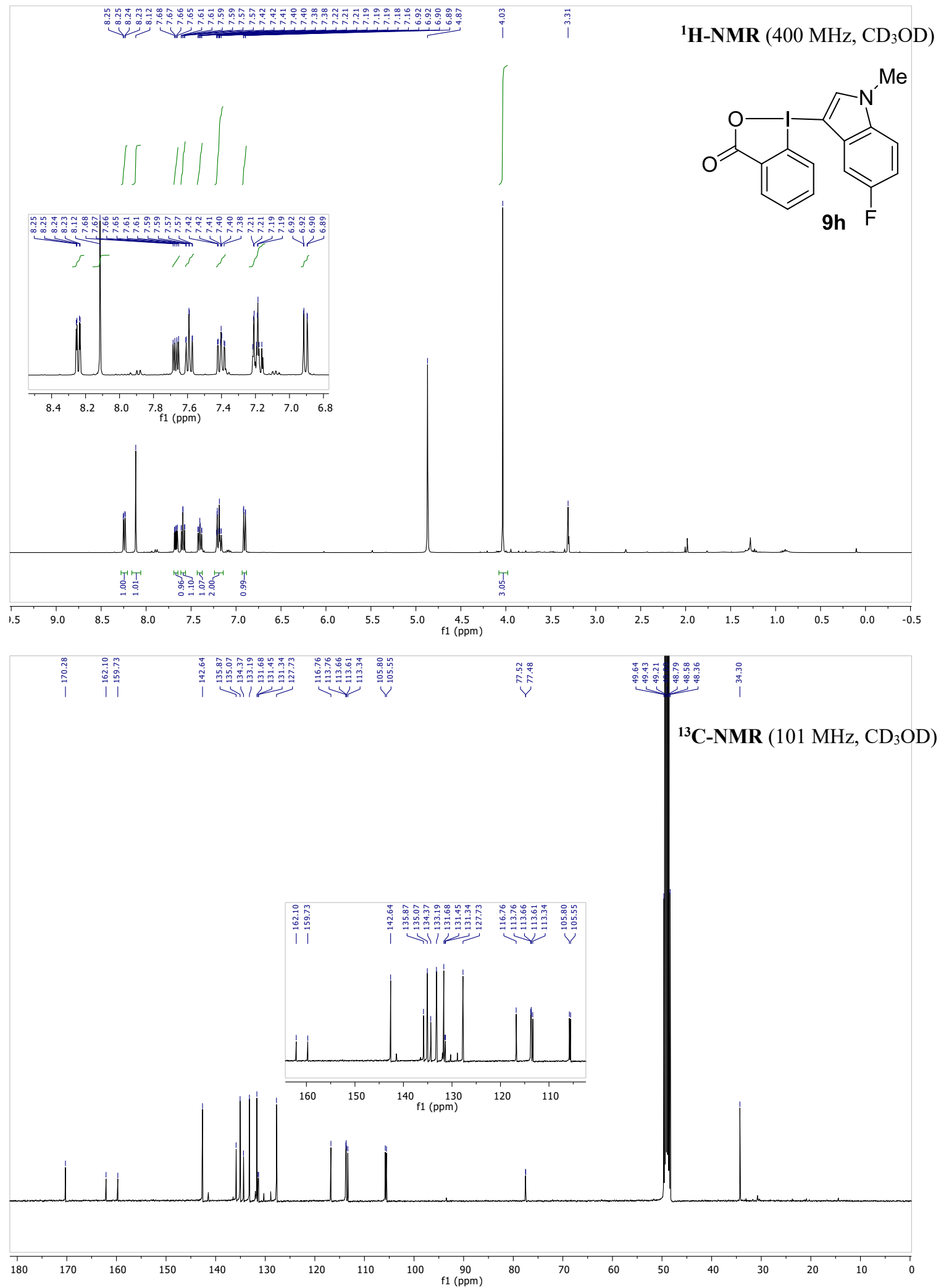


1-(3-5-Chloro-1-methyl-1 $H$-indole)-1 $H$-1 $\lambda_{3}$-benzo[b]iodo-3(2H)-one (9i)

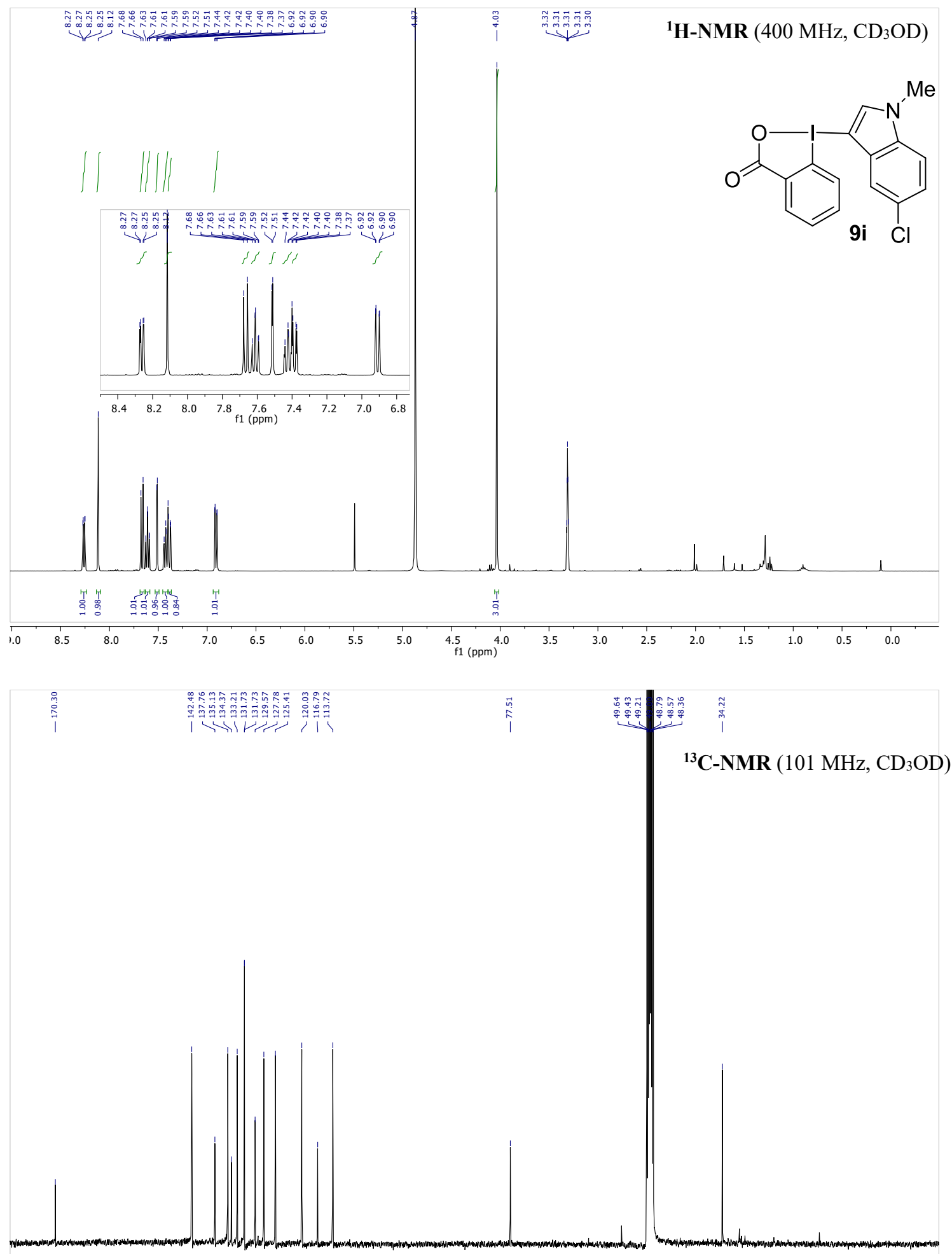

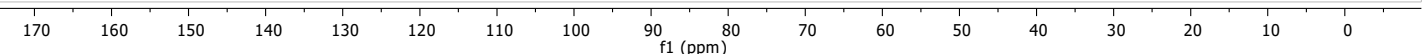


1-(3-5-Iodo-1-methyl-1 $H$-indole)-1H-1 $\lambda_{3}$-benzo $[b]$ iodo-3(2H)-one (9j)

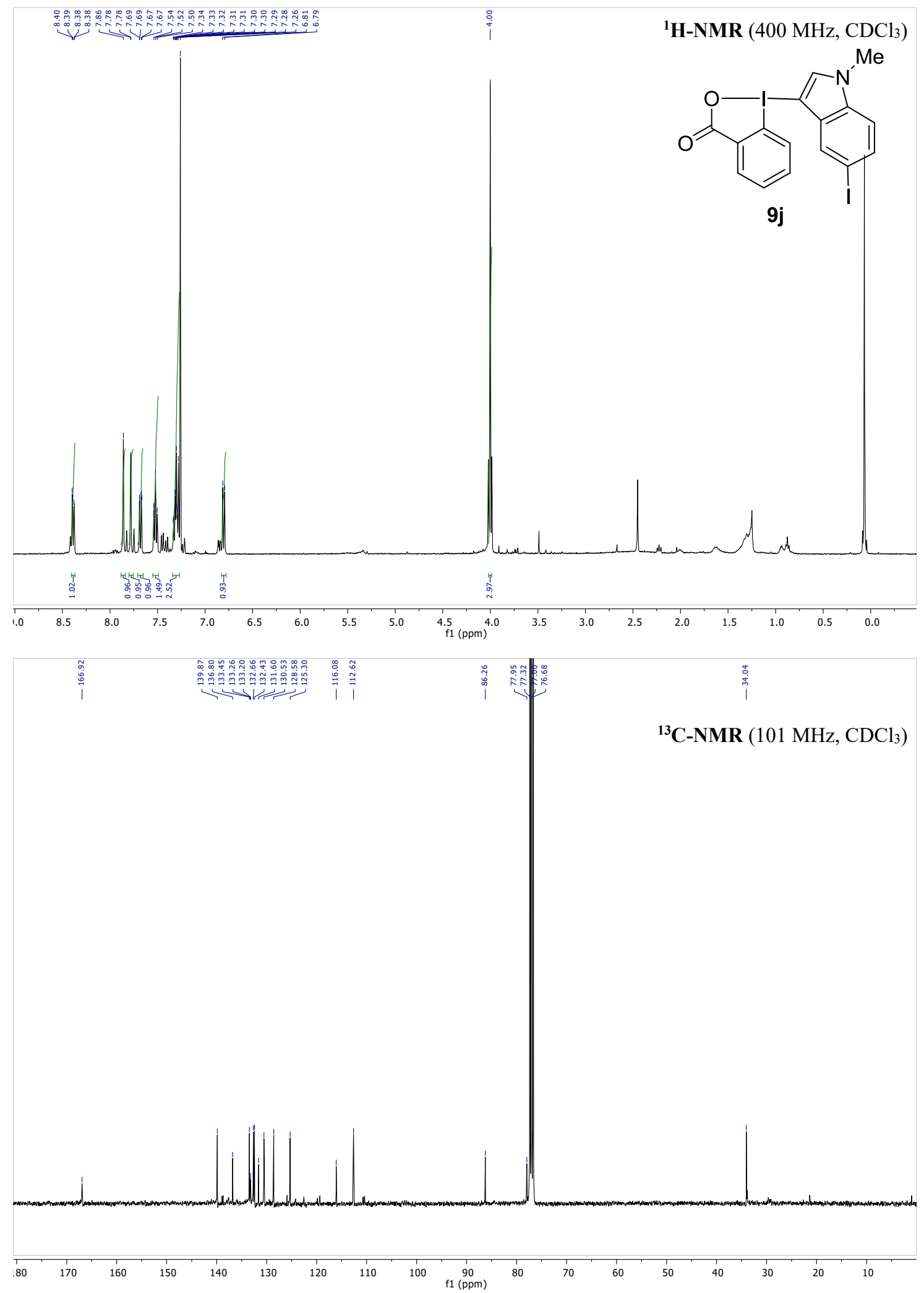


1-(3-1-Methyl-5-(4,4,5,5-tetramethyl-1,3,2-dioxaborolan-2-yl)-1H-indole)-1H-1 $\lambda_{3}$-benzo $[b]$ iodo$3(2 H)$-one (9k)

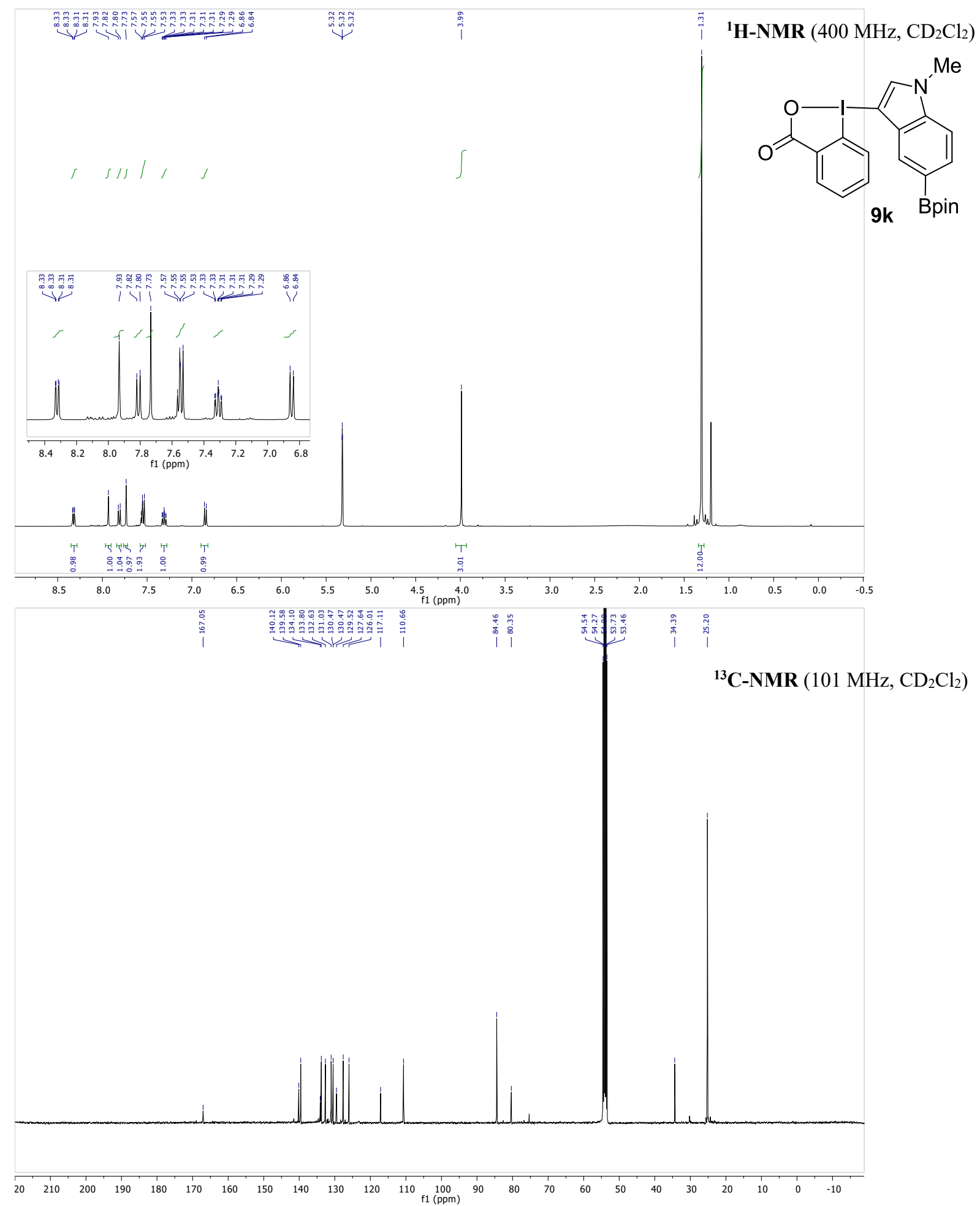


1-(3-1-Methyl-1 $H$-pyrrolo[2,3-b]pyridine)-1H-1 $\lambda_{3}$-benzo[b]iodo-3(2H)-one (91)

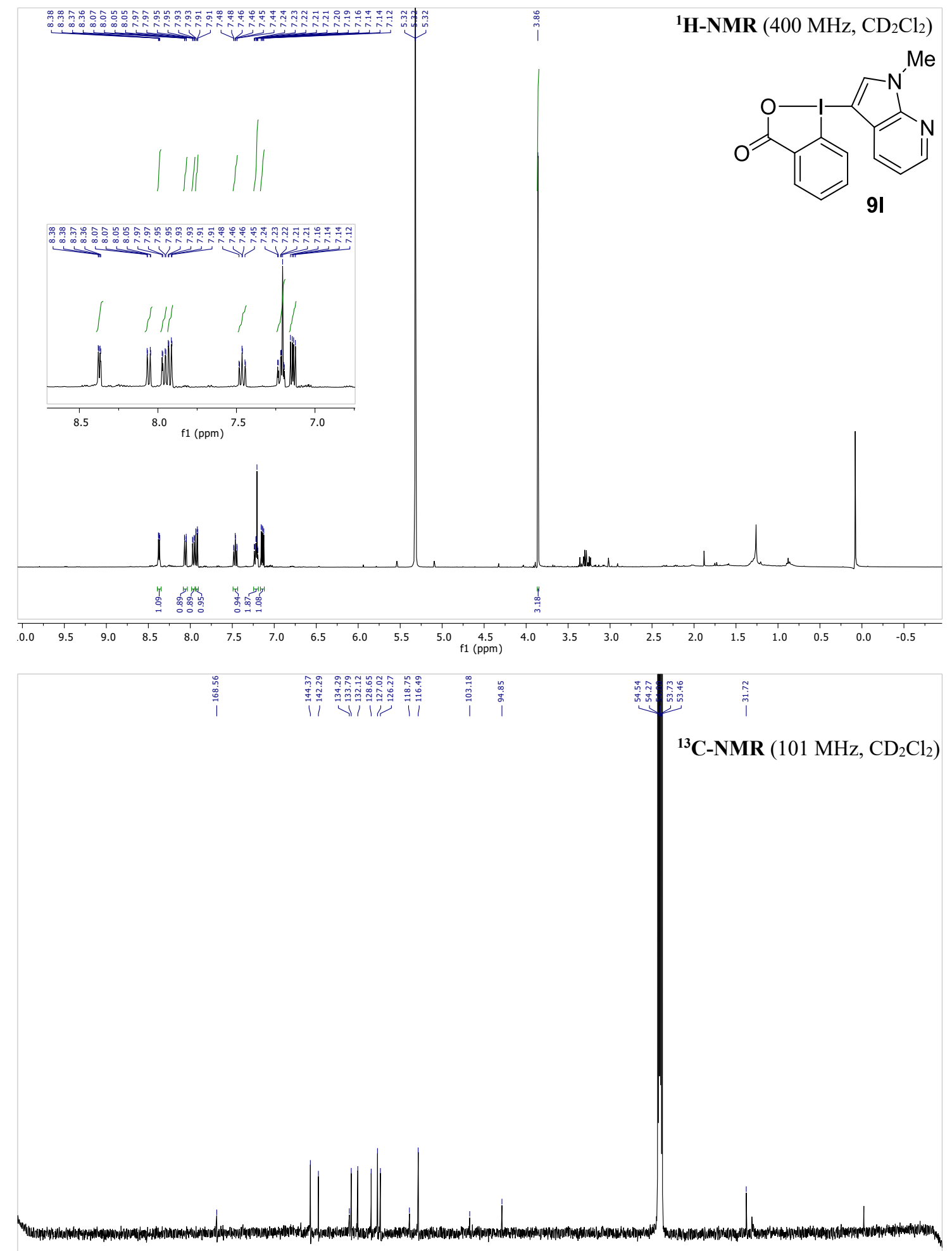

\begin{tabular}{llllllllllllllllllllllllllllll}
\hline & 210 & 200 & 190 & 180 & 170 & 160 & 150 & 140 & 130 & 120 & 110 & 100 & 90 & 80 & 70 & 60 & 50 & 40 & 30 & 20 & 10 & 0 & -10 & 1
\end{tabular} 
1-(3-1H-Pyrrole)-1H-1 $1 \lambda_{3}$-benzo[b]iodo-3(2H)-one (9m)

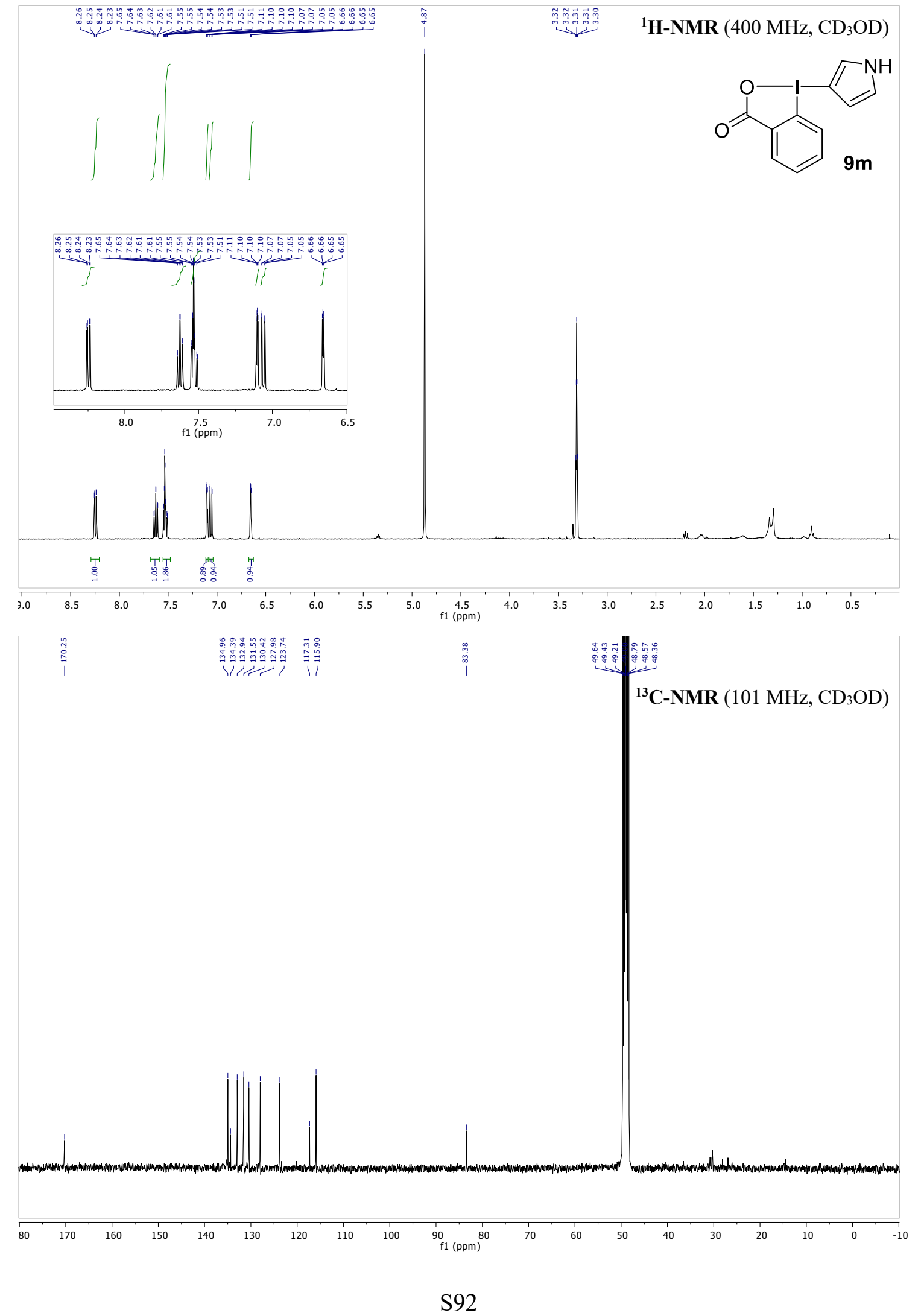


1-(3-1-Methyl-1H-pyrrole)-1H-1 $\lambda_{3}$-benzo[b]iodo-3(2H)-one (9n)

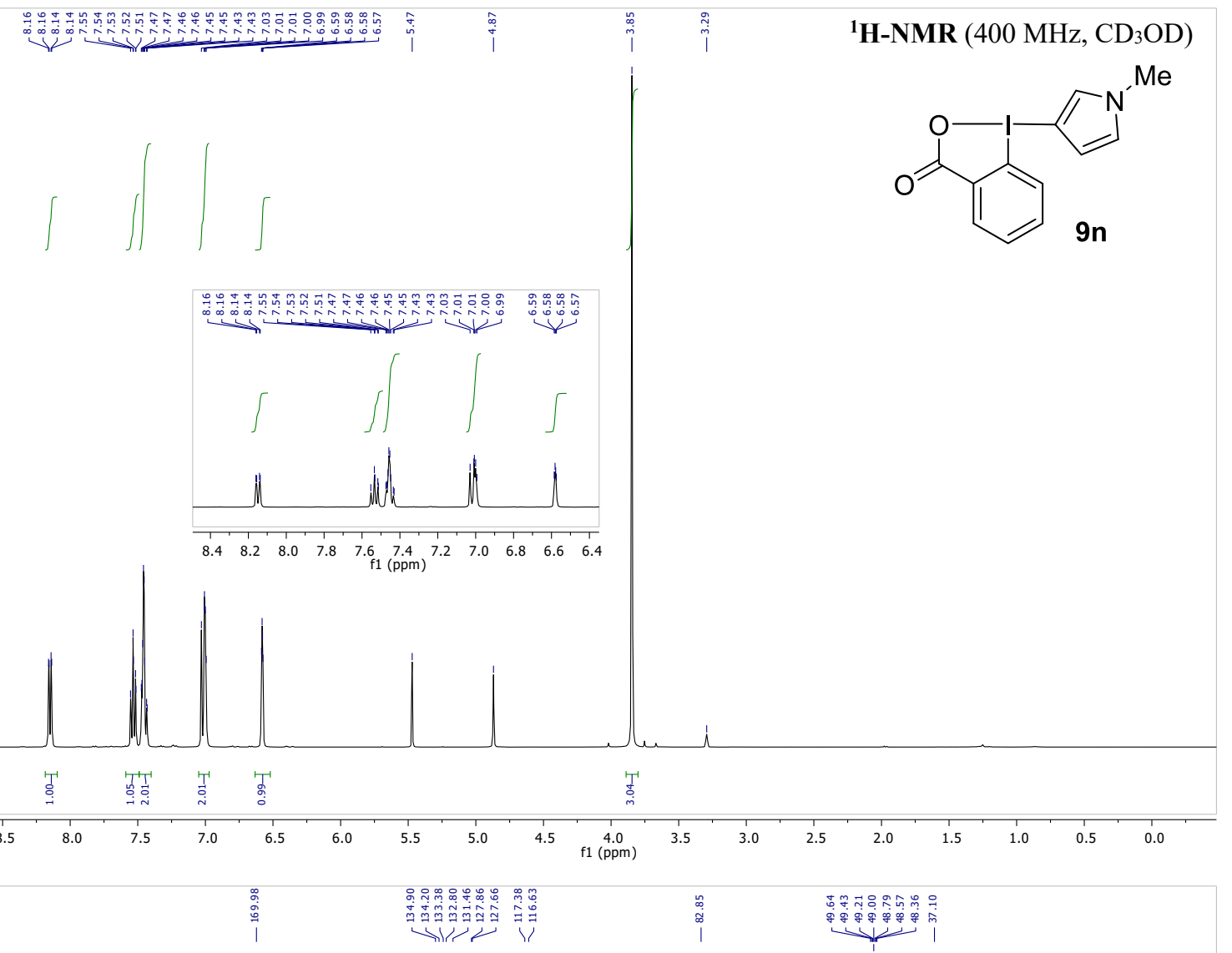

${ }^{13}$ C-NMR (101 MHz, $\left.\mathrm{CD}_{3} \mathrm{OD}\right)$

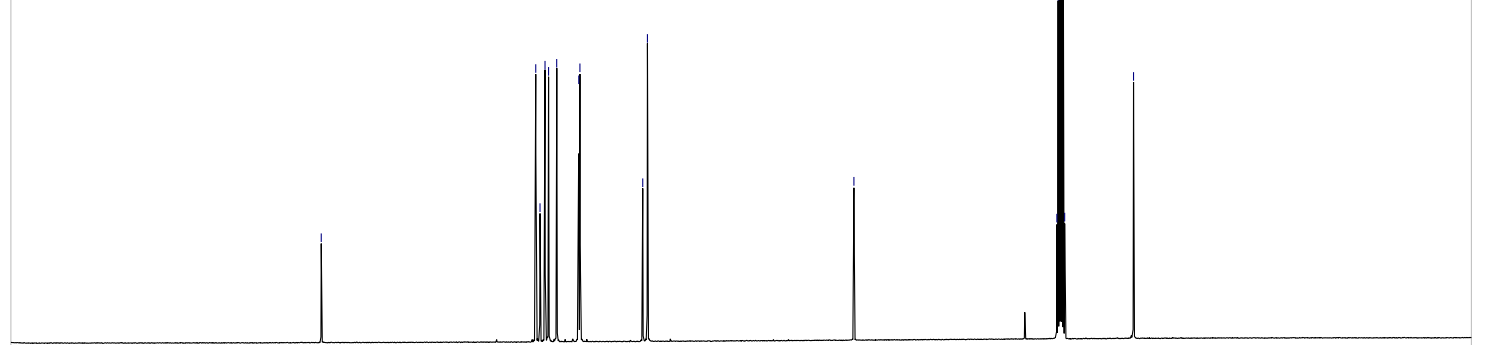

$\begin{array}{llllllllllllllllllllllllllllll}1 & 20 & 210 & 200 & 190 & 180 & 170 & 160 & 150 & 140 & 130 & 120 & 110 & 100 & 90 & 80 & 70 & 60 & 50 & 40 & 30 & 20 & 10 & 0 & -10\end{array}$ 
1-(2-1-Methyl-1H-pyrrole)-1H-1 $\lambda_{3}$-benzo[b]iodo-3(2H)-one (9o)
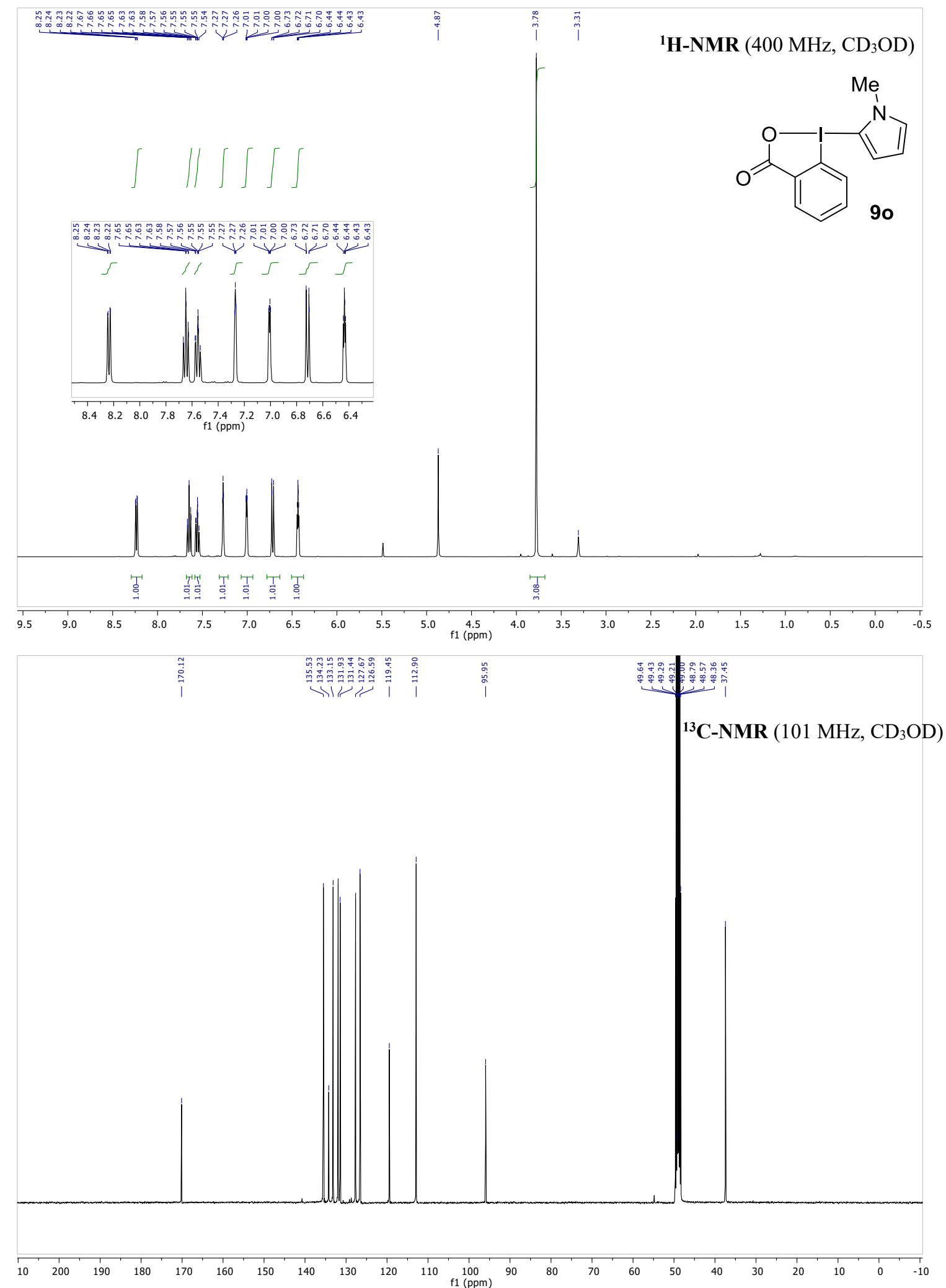
1-(3-1-Benzyl-1H-pyrrole)-1 $H$-1 $1 \lambda_{3}$-benzo[b]iodo-3(2H)-one (9p)
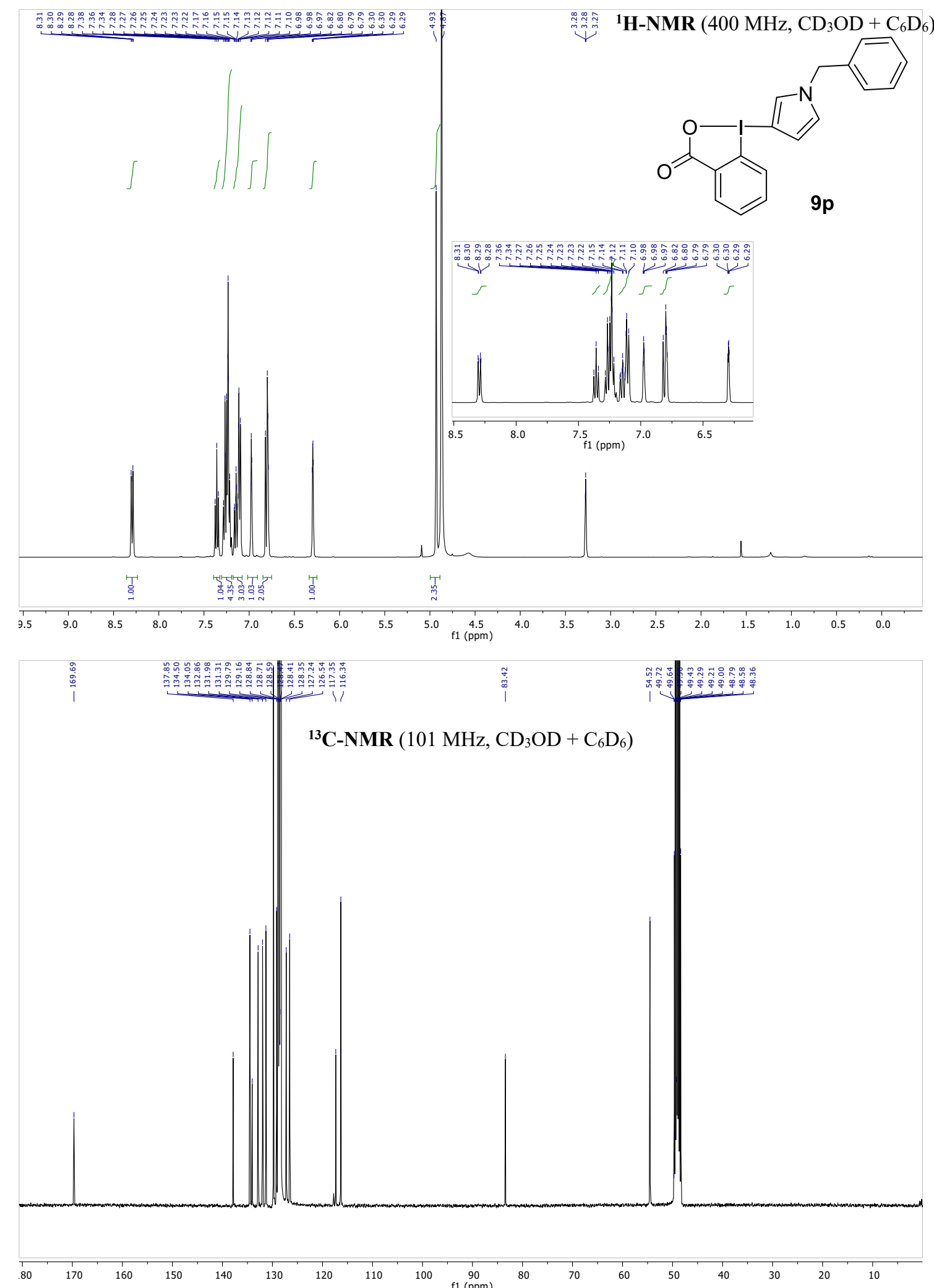
1-(2-1-Benzyl-1 $H$-pyrrole)-1 $H$-1 $\lambda_{3}$-benzo[b]iodo-3(2H)-one (9q)
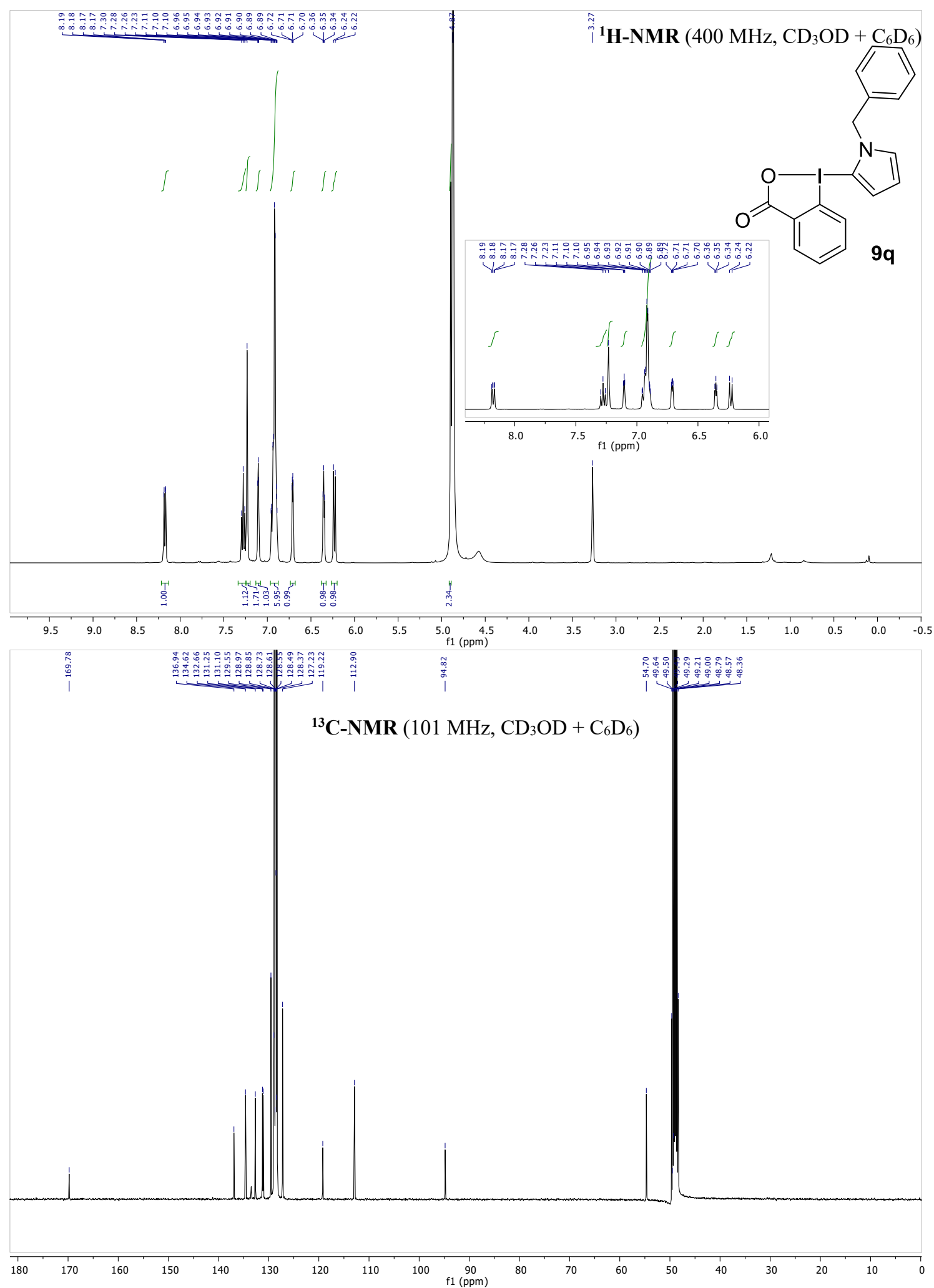
1-(3-9-Methyl-9H-carbazole)-1H-1 $\lambda_{3}$-benzo[b]iodo-3(2H)-one (9r)

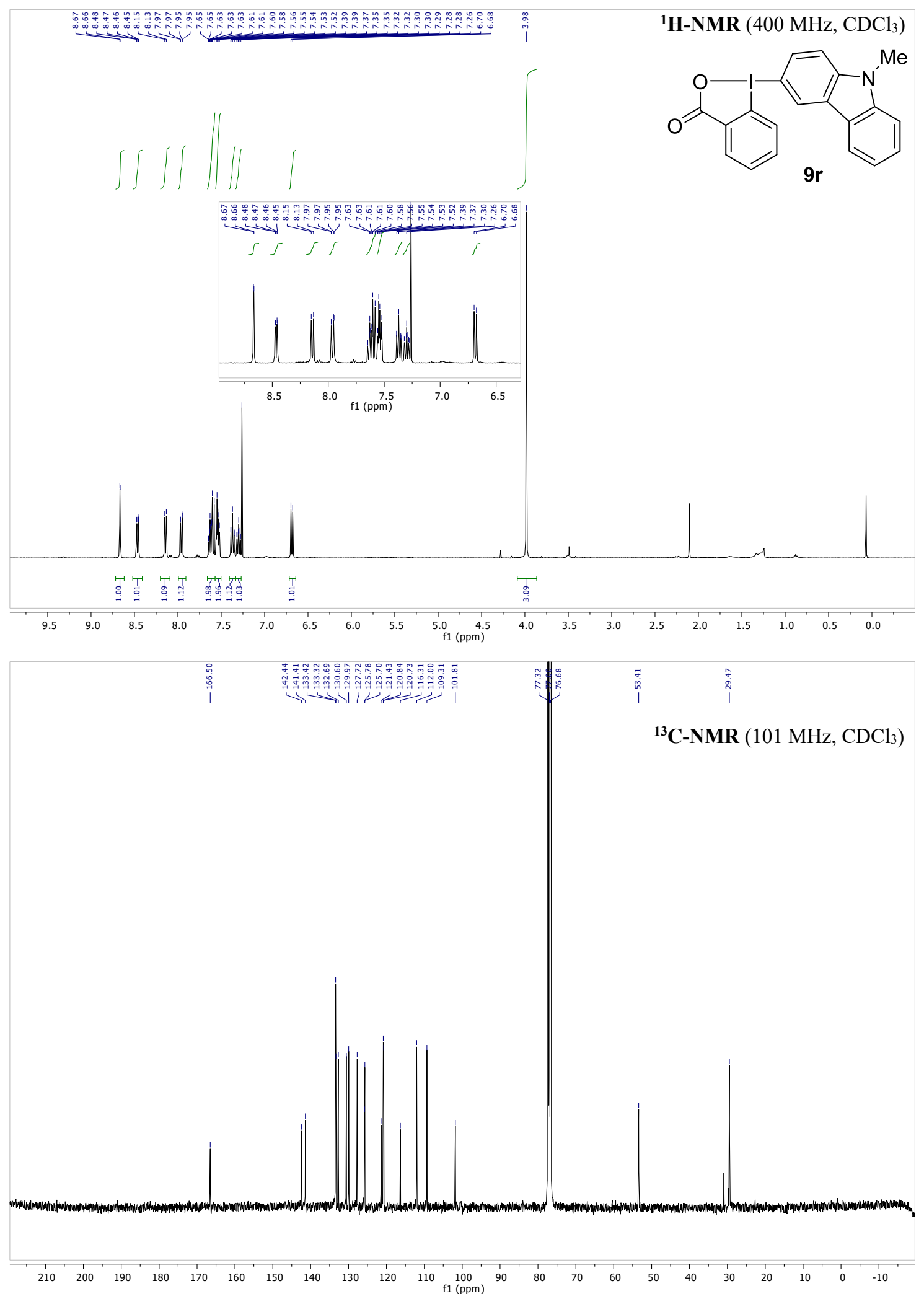


1-(2-1H-Thiophene)-1H-1 $\lambda_{3}$-benzo[b]iodo-3(2H)-one (9s)
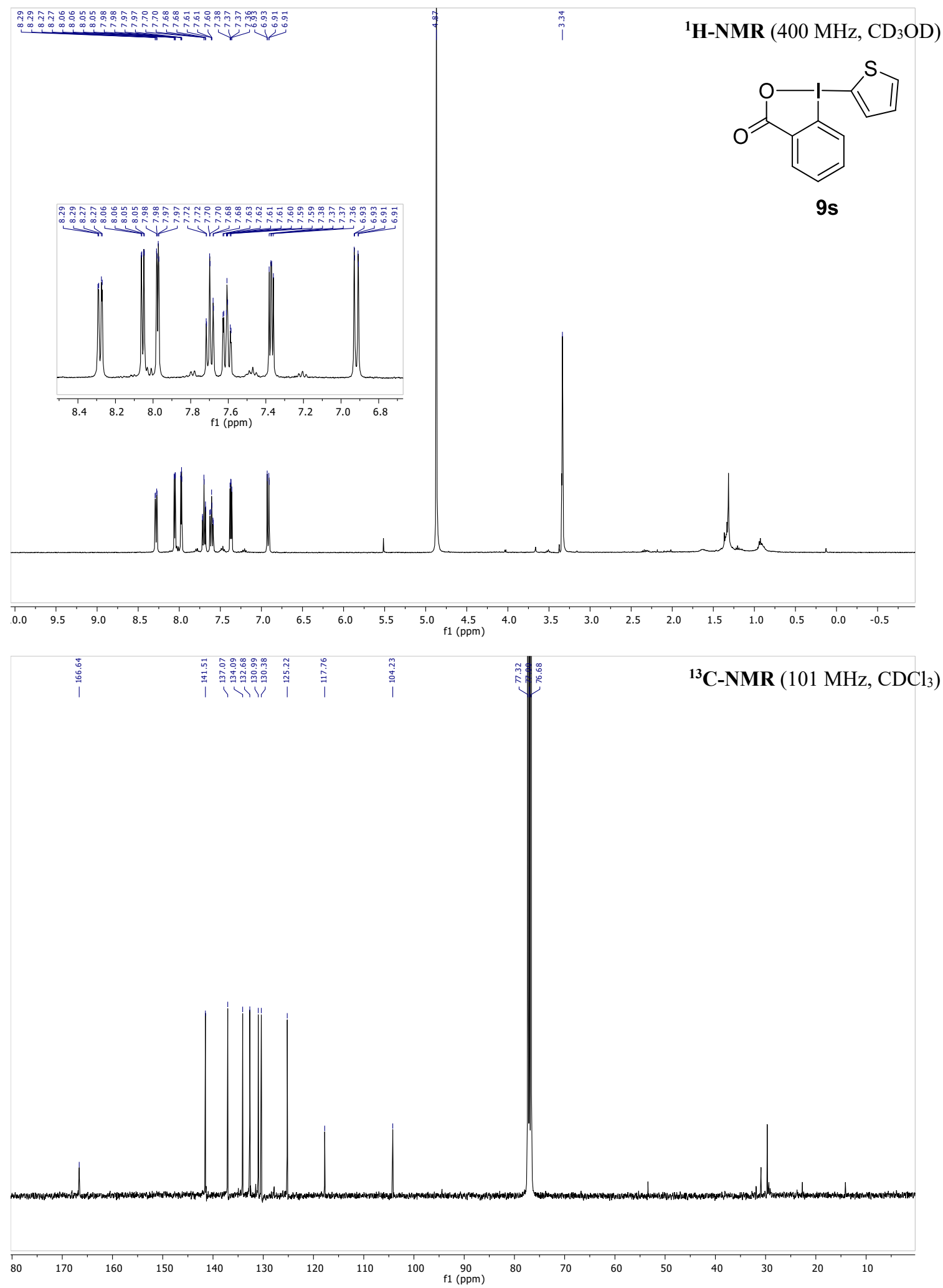
1-(3-1H-2,5-dimethylfuran)-1H-1 $\lambda_{3}$-benzo[b]iodo-3(2H)-one (9t)

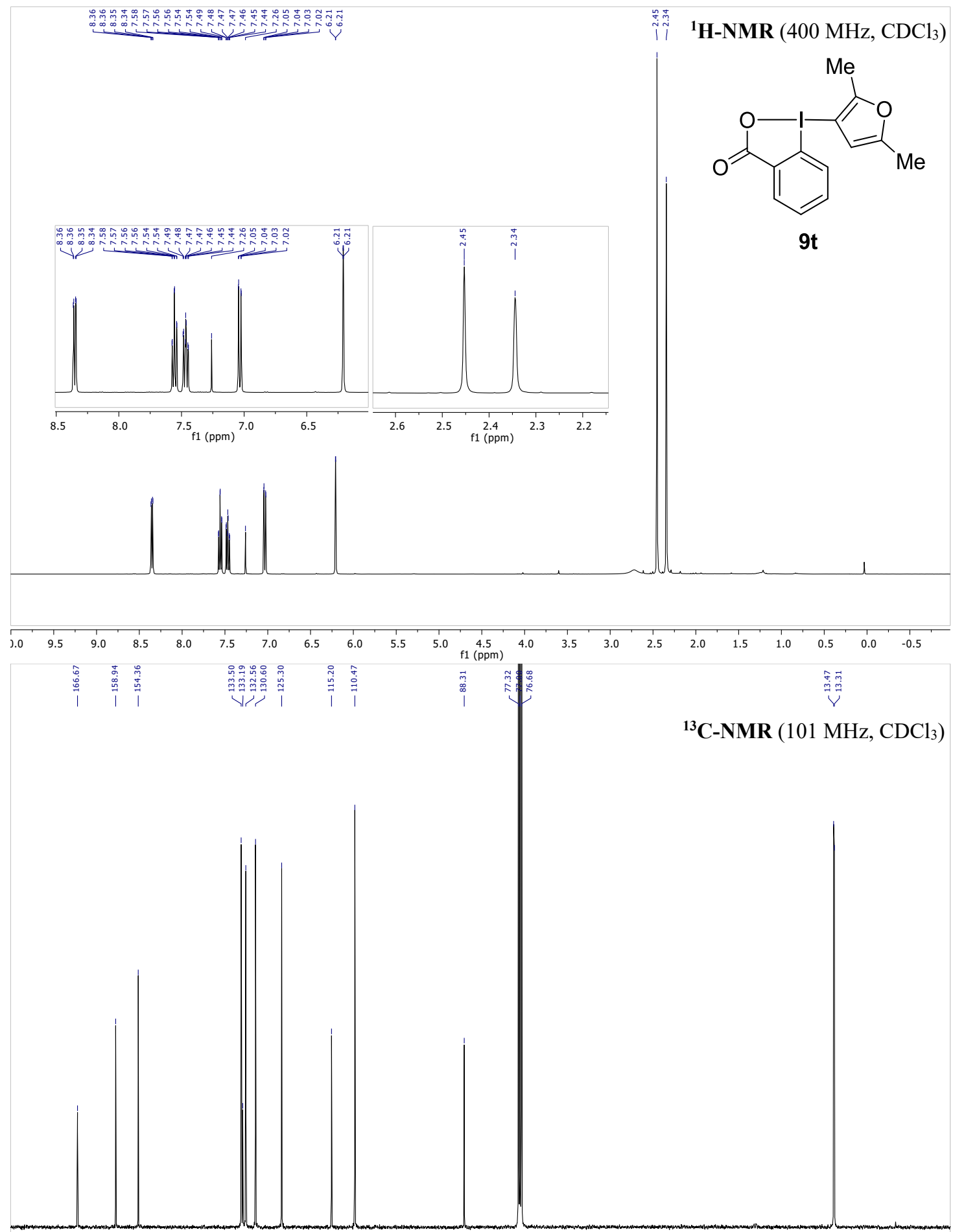

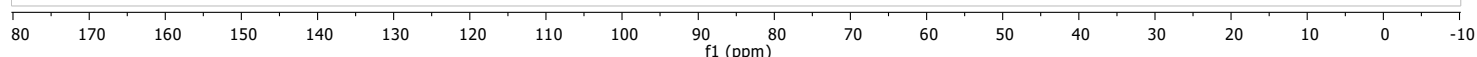

ß-Phenyliodonioindole Tetrafluoroborate (18). 


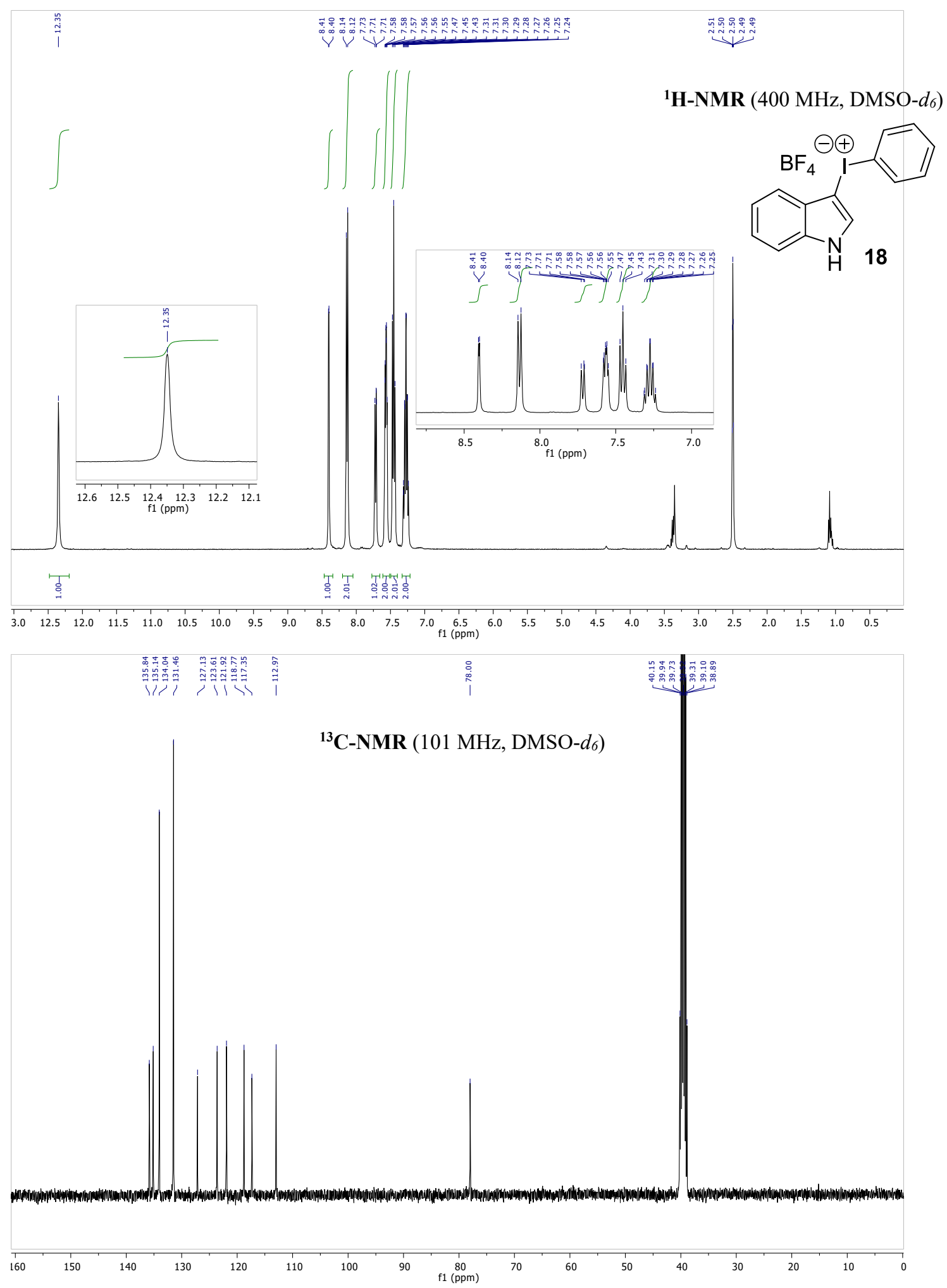

1-Methyl-3-(2-(pyridin-2-yl)phenyl)-1 H-indole (13a) 


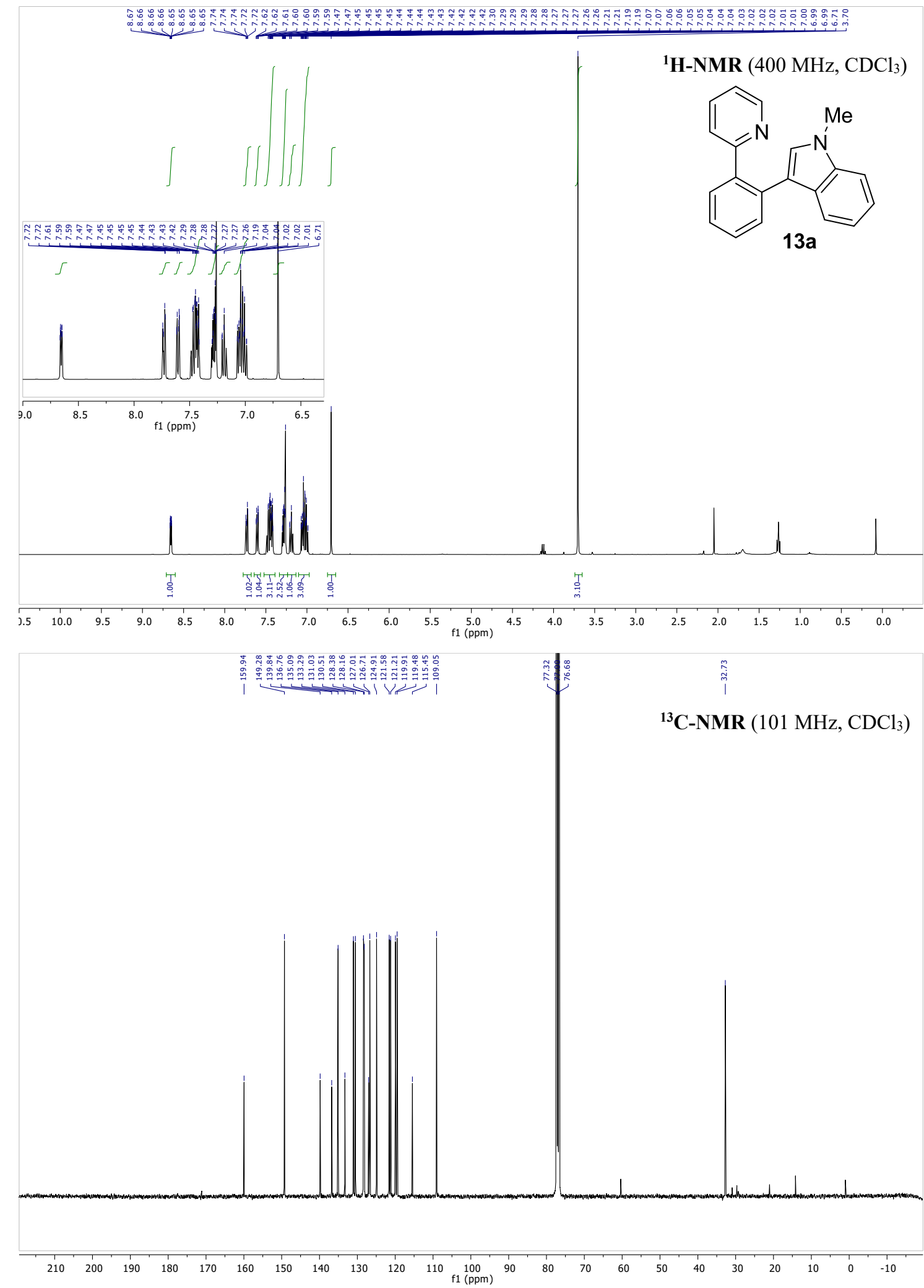

3-(2-(Pyridin-2-yl)phenyl)-1 H-indole (13b) 


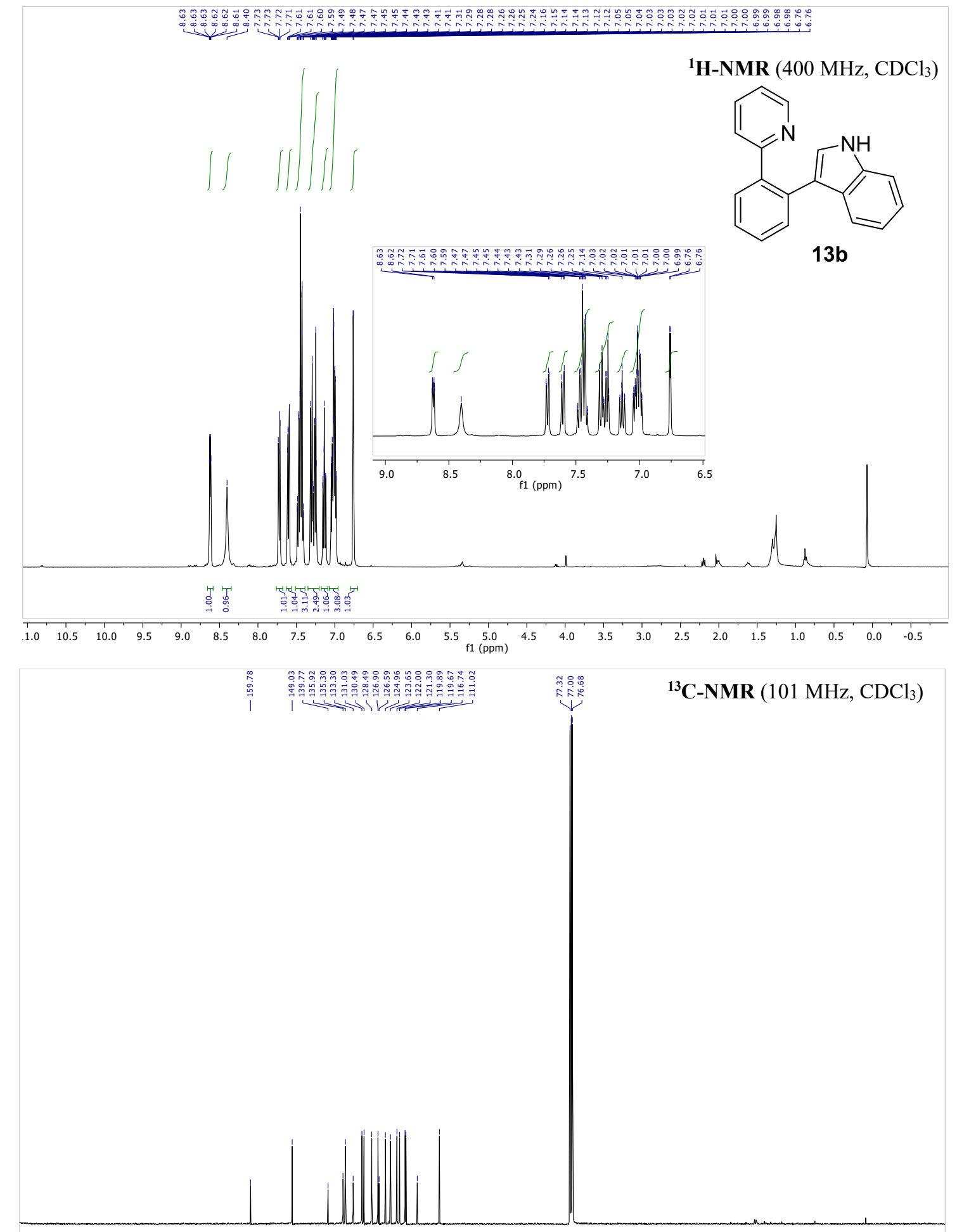

$\begin{array}{lllllllllllllllllllllllllllllllll}210 & 200 & 190 & 180 & 170 & 160 & 150 & 140 & 130 & 120 & 110 & 100 & 90 & 80 & 70 & 60 & 50 & 40 & 30 & 20 & 10 & 0 & -10\end{array}$

1-(3-Phenylpropyl)-3-(2-(pyridin-2-yl)phenyl)-1H-indole (13c) 

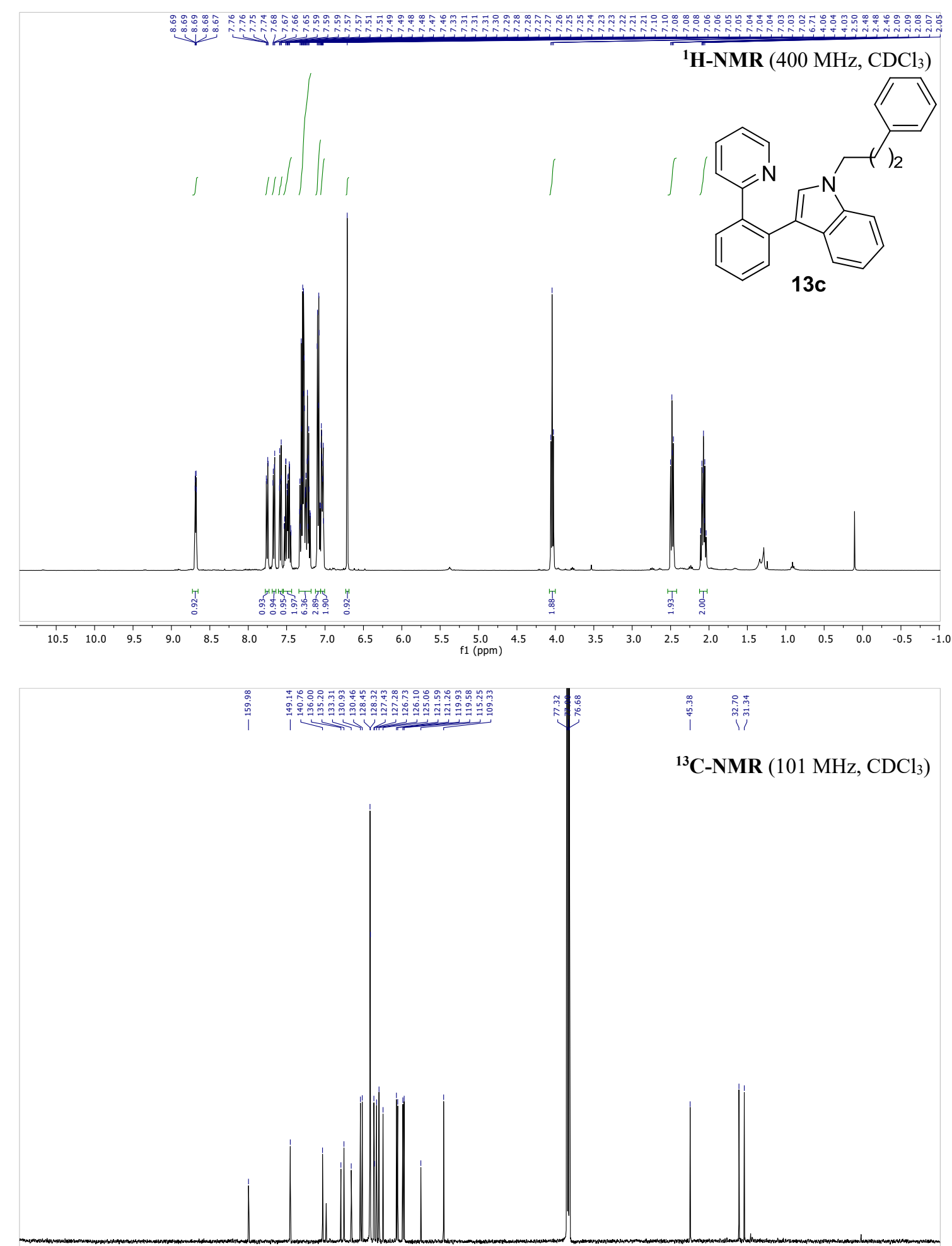

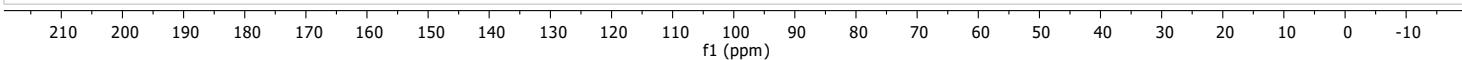

5-Methoxy-1-methyl-3-(2-(pyridin-2-yl)phenyl)-1H-indole (13d) 

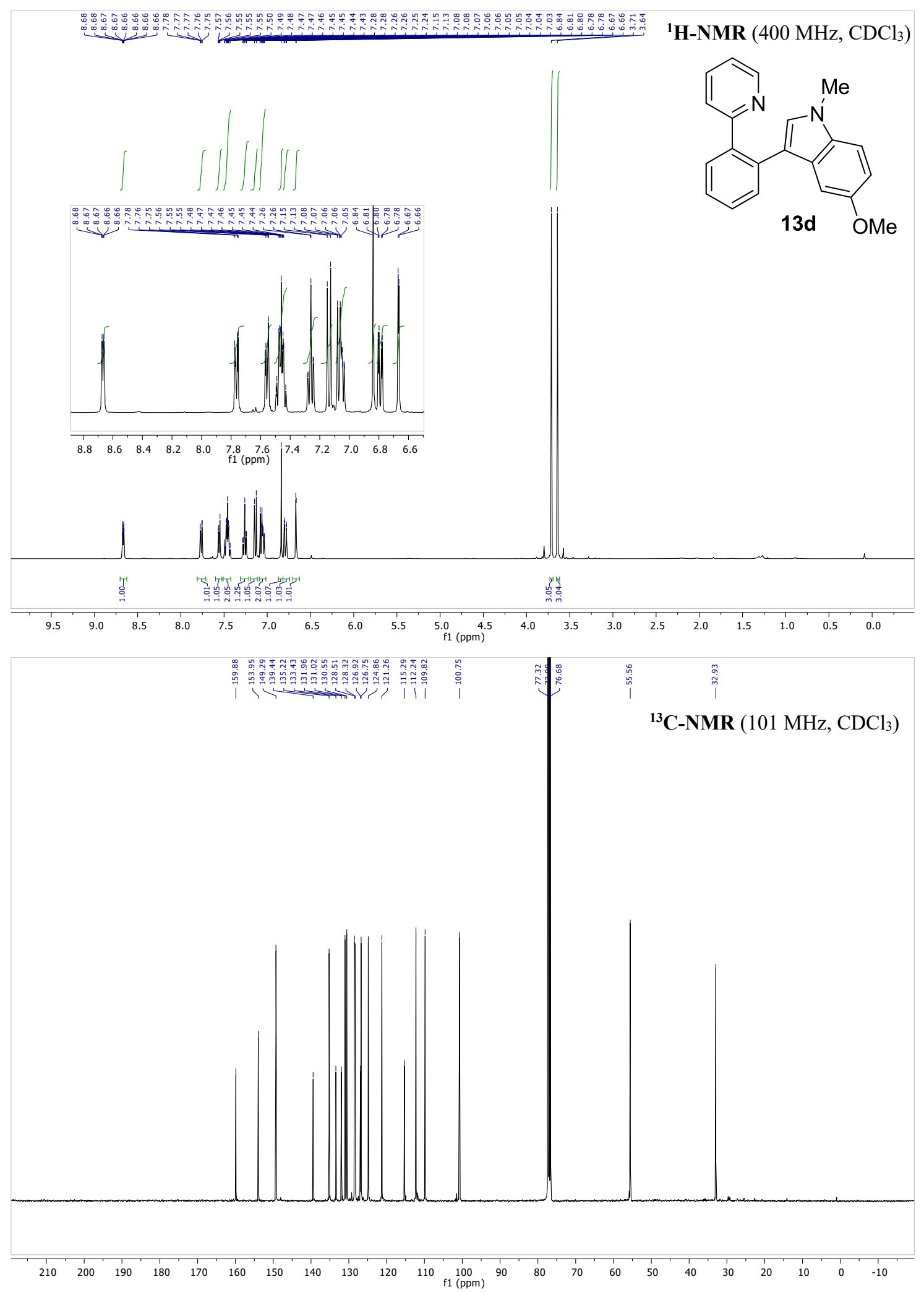

5-Fluoro-1-methyl-3-(2-(pyridin-2-yl)phenyl)-1H-indole (13e)

${ }^{1} \mathbf{H}-\mathbf{N M R}\left(400 \mathrm{MHz}, \mathrm{CDCl}_{3}\right.$ ) 

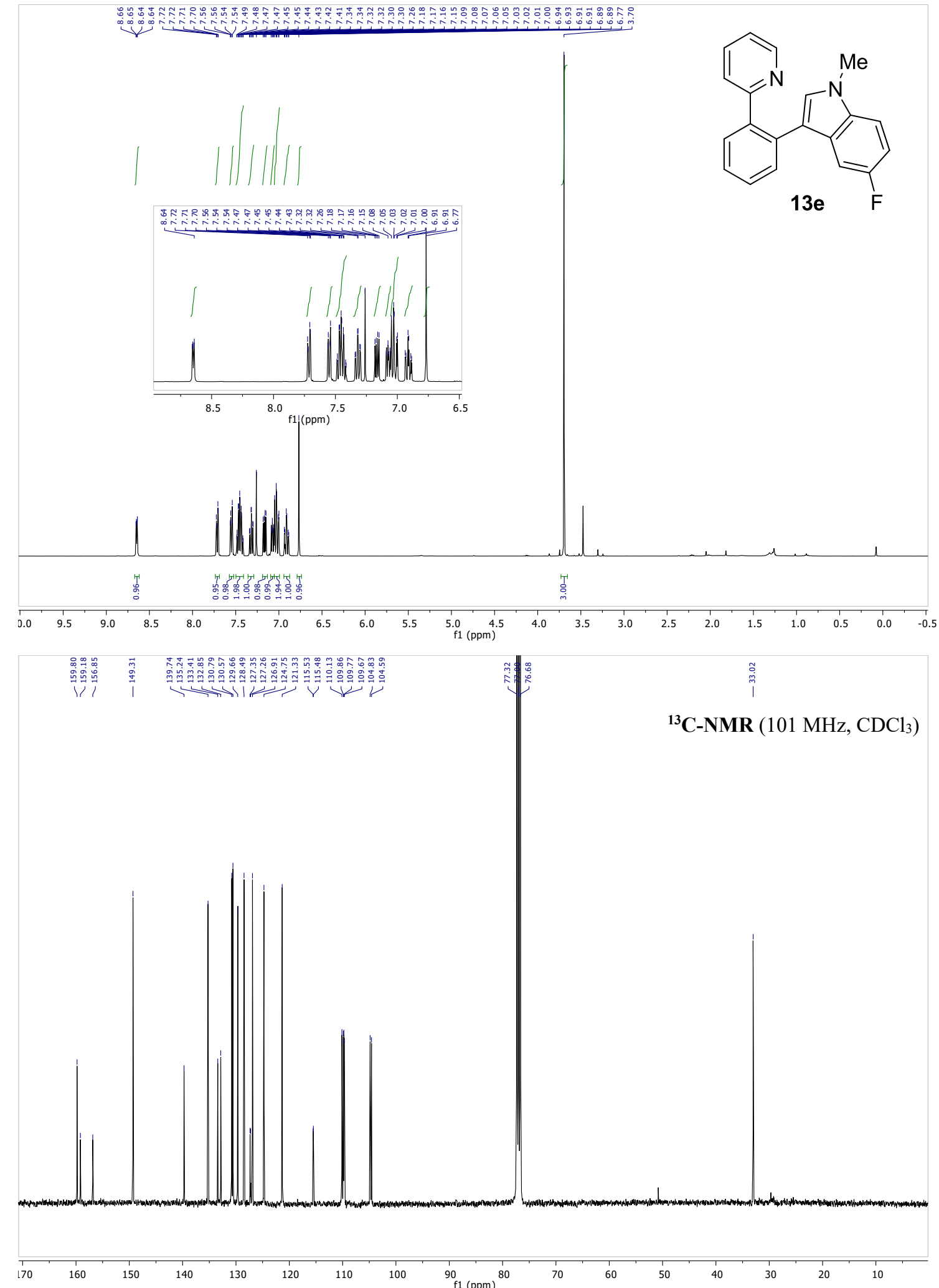

5-Chloro-1-methyl-3-(2-(pyridin-2-yl)phenyl)-1H-indole (13f) 


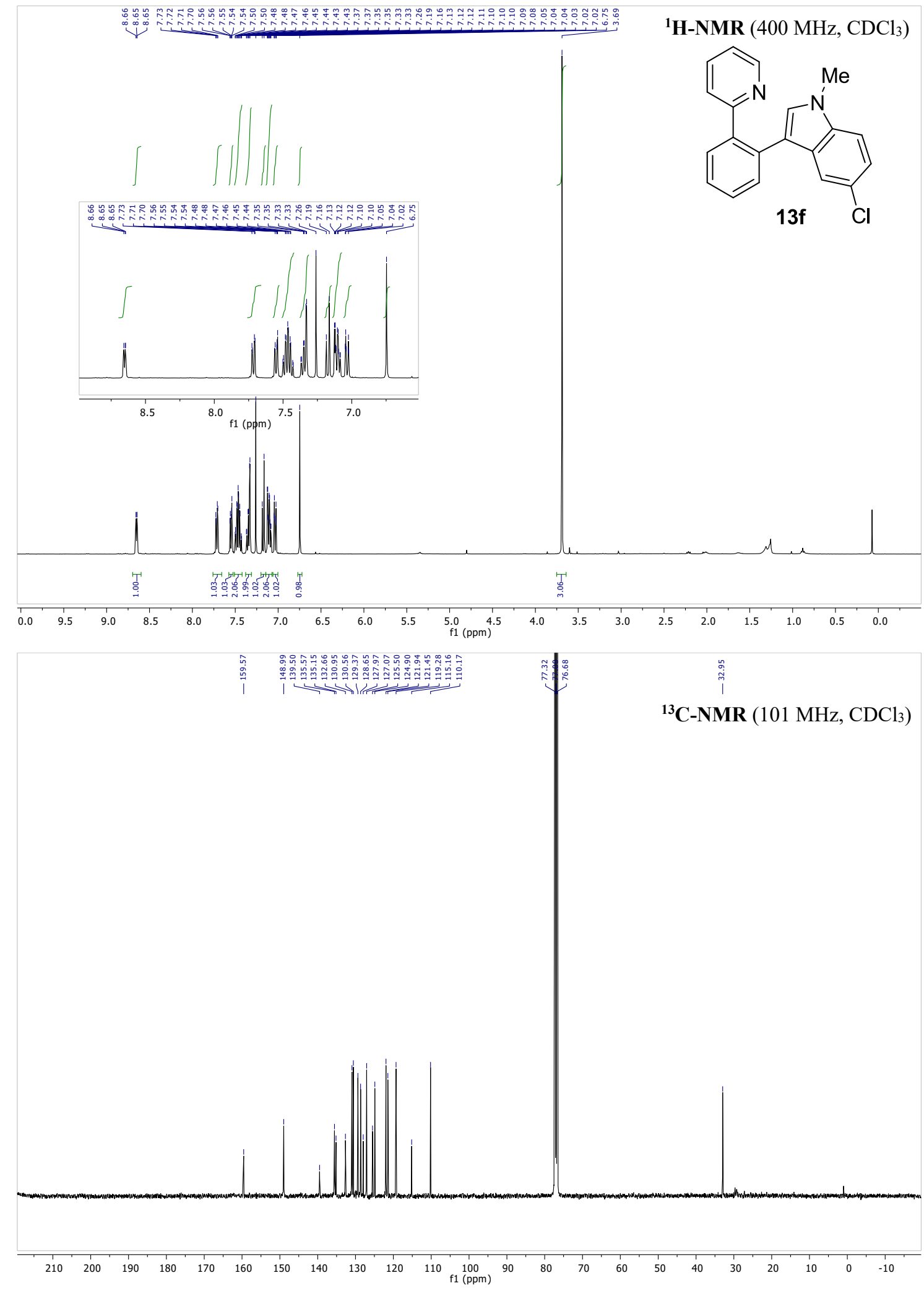

5-Iodo-1-methyl-3-(2-(pyridin-2-yl)phenyl)-1H-indole (13g) 
${ }^{1} \mathbf{H}-\mathbf{N M R}\left(400 \mathrm{MHz}, \mathrm{CDCl}_{3}\right)$
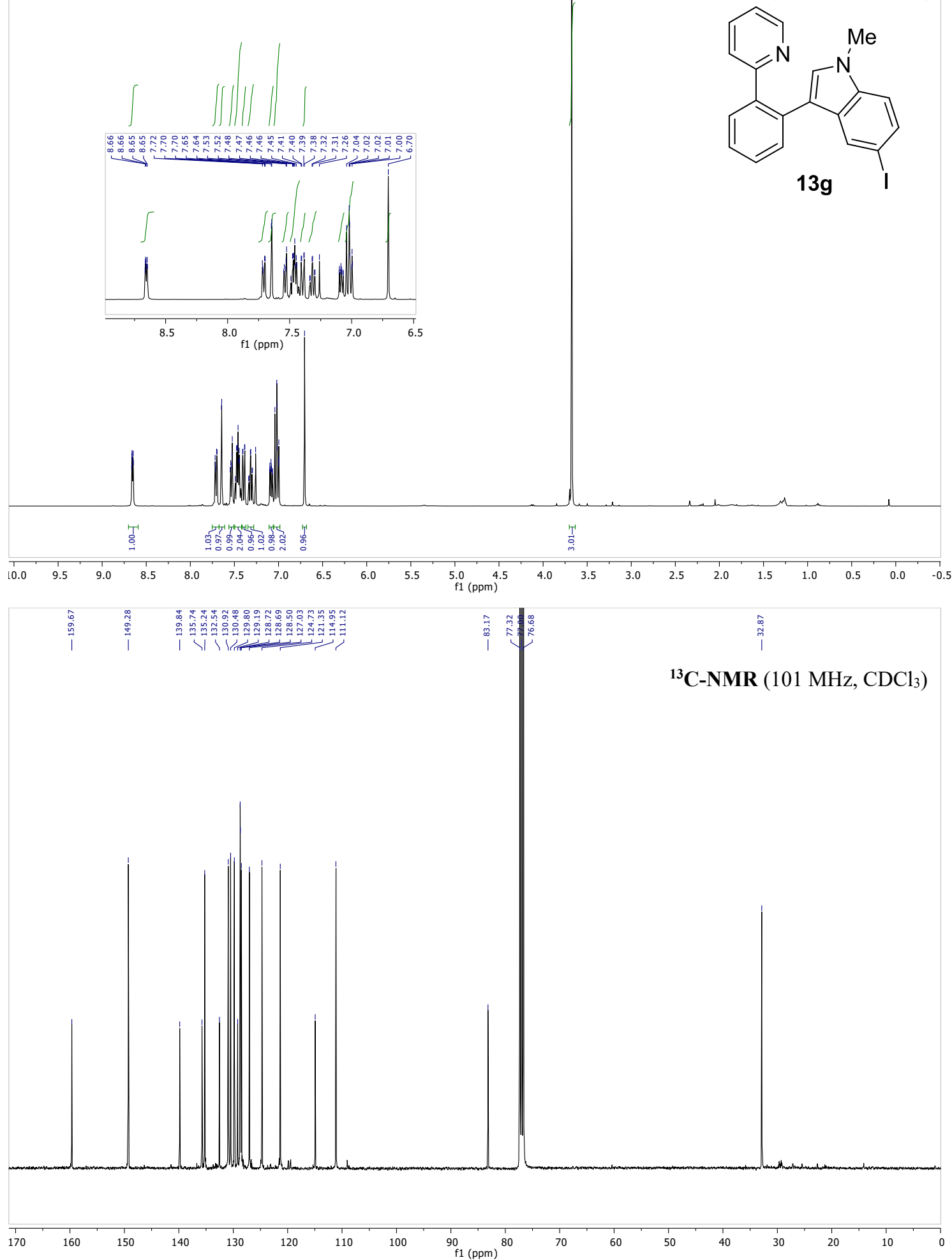
1-Methyl-3-(2-(pyridin-2-yl)phenyl)-5-(4,4,5,5-tetramethyl-1,3,2-dioxaborolan-2-yl)-1 Hindole (13h)

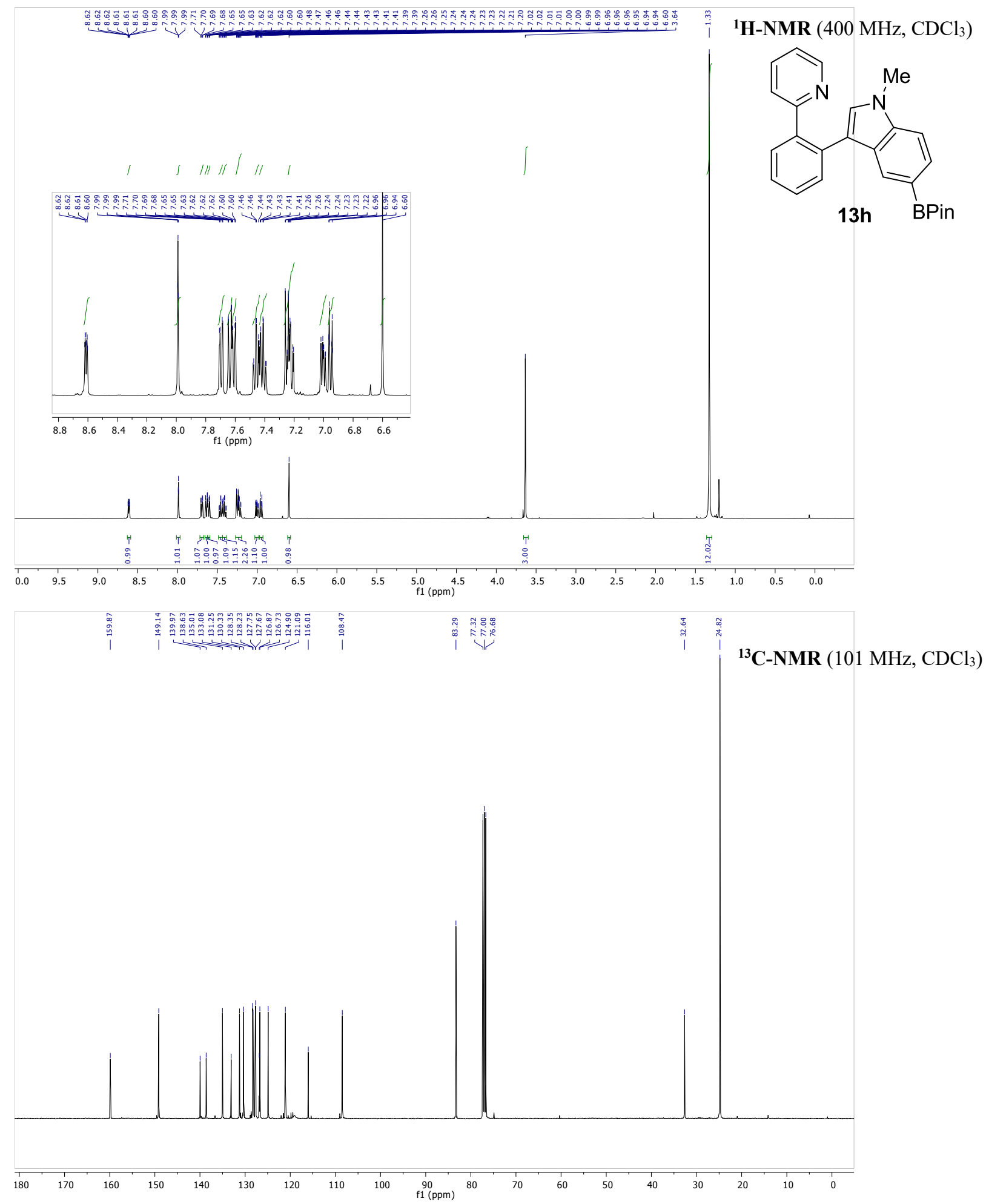




\section{2-(2-(1-Methyl-1H-pyrrol-3-yl)phenyl)pyridine (13i)}
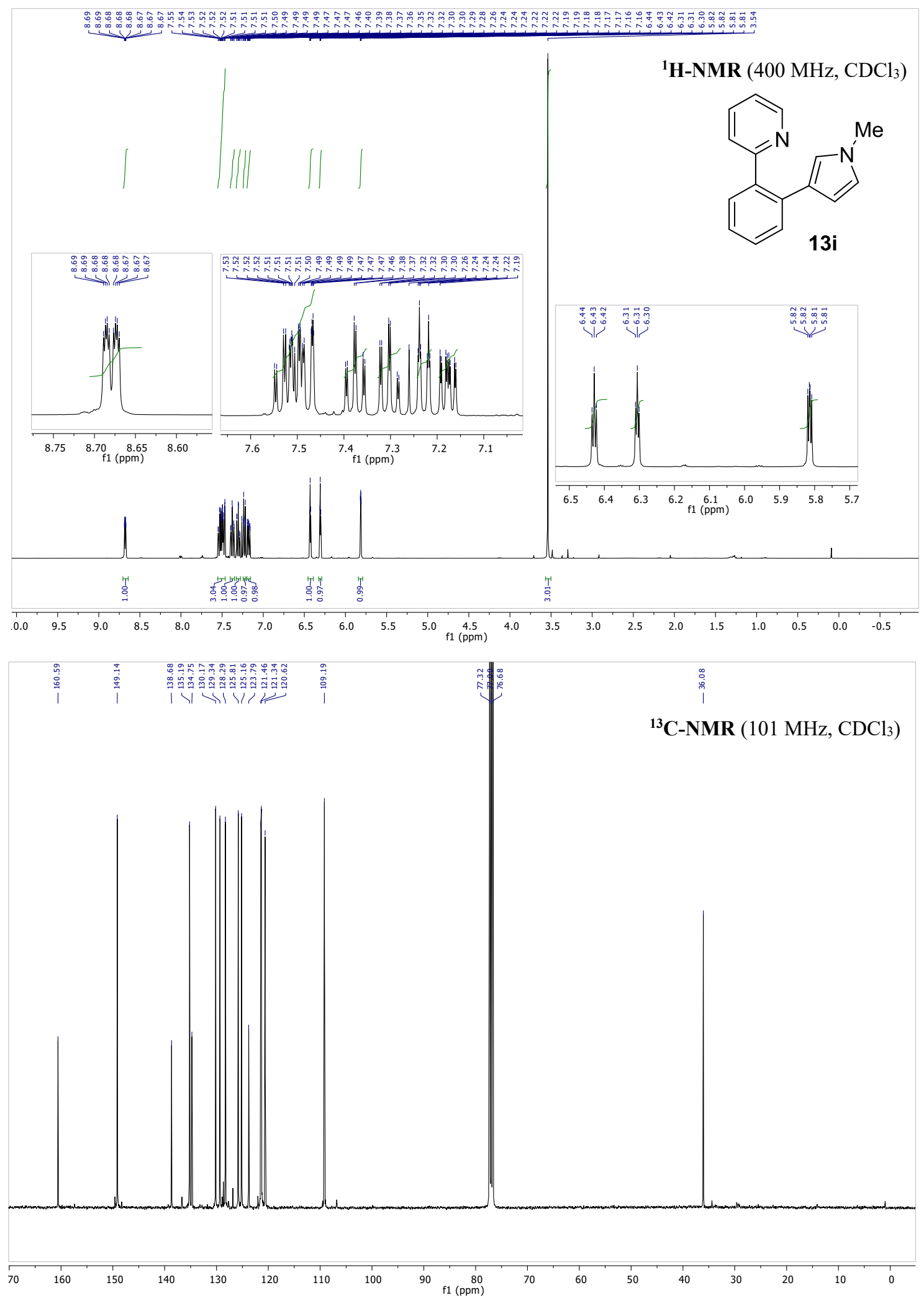
$\underbrace{\prime}$
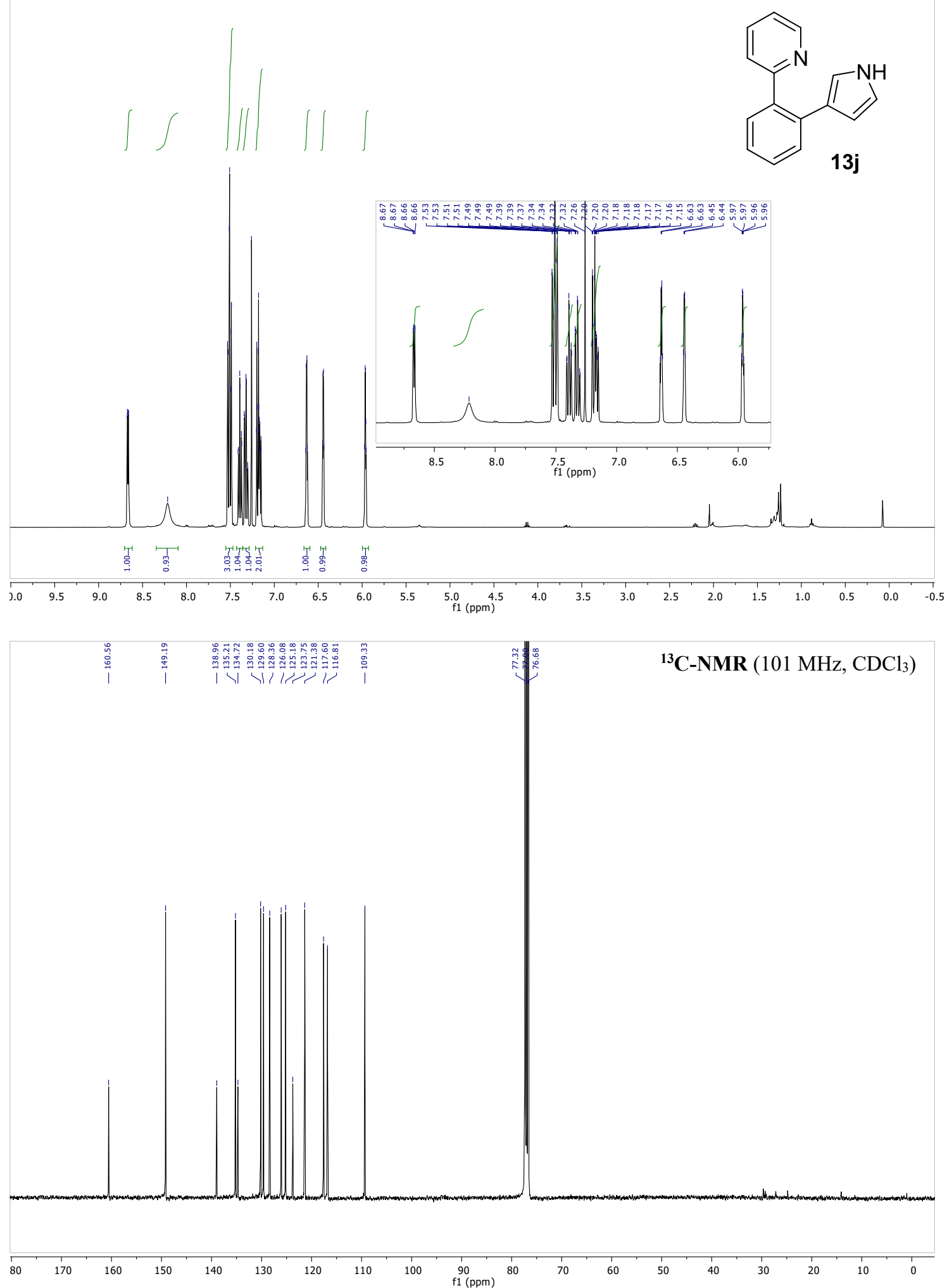


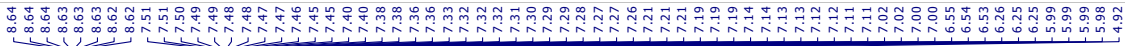
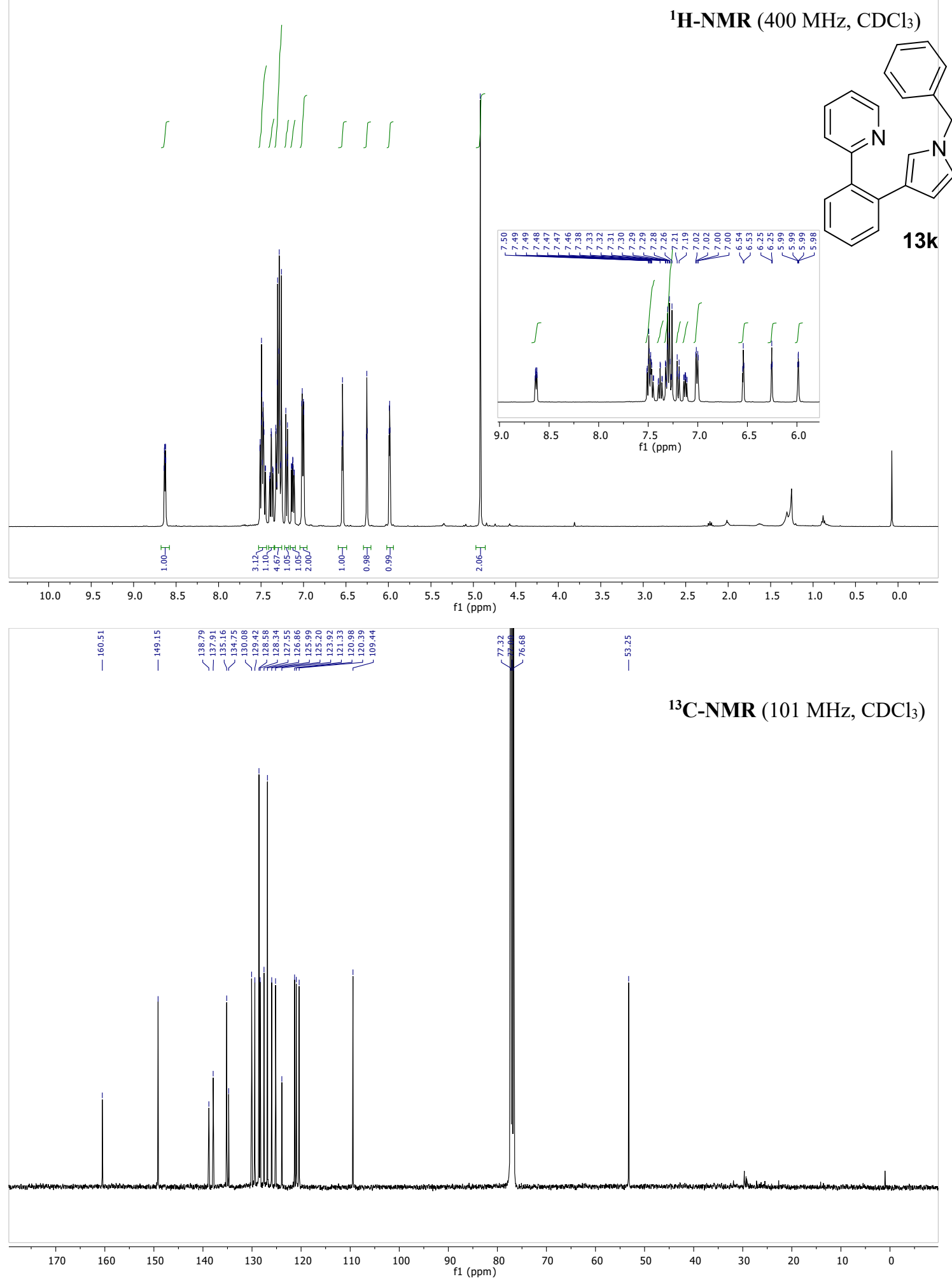
2-(2-(1-Methyl-1H-pyrrol-2-yl)phenyl)pyridine (13l)

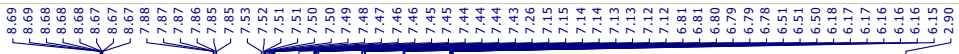
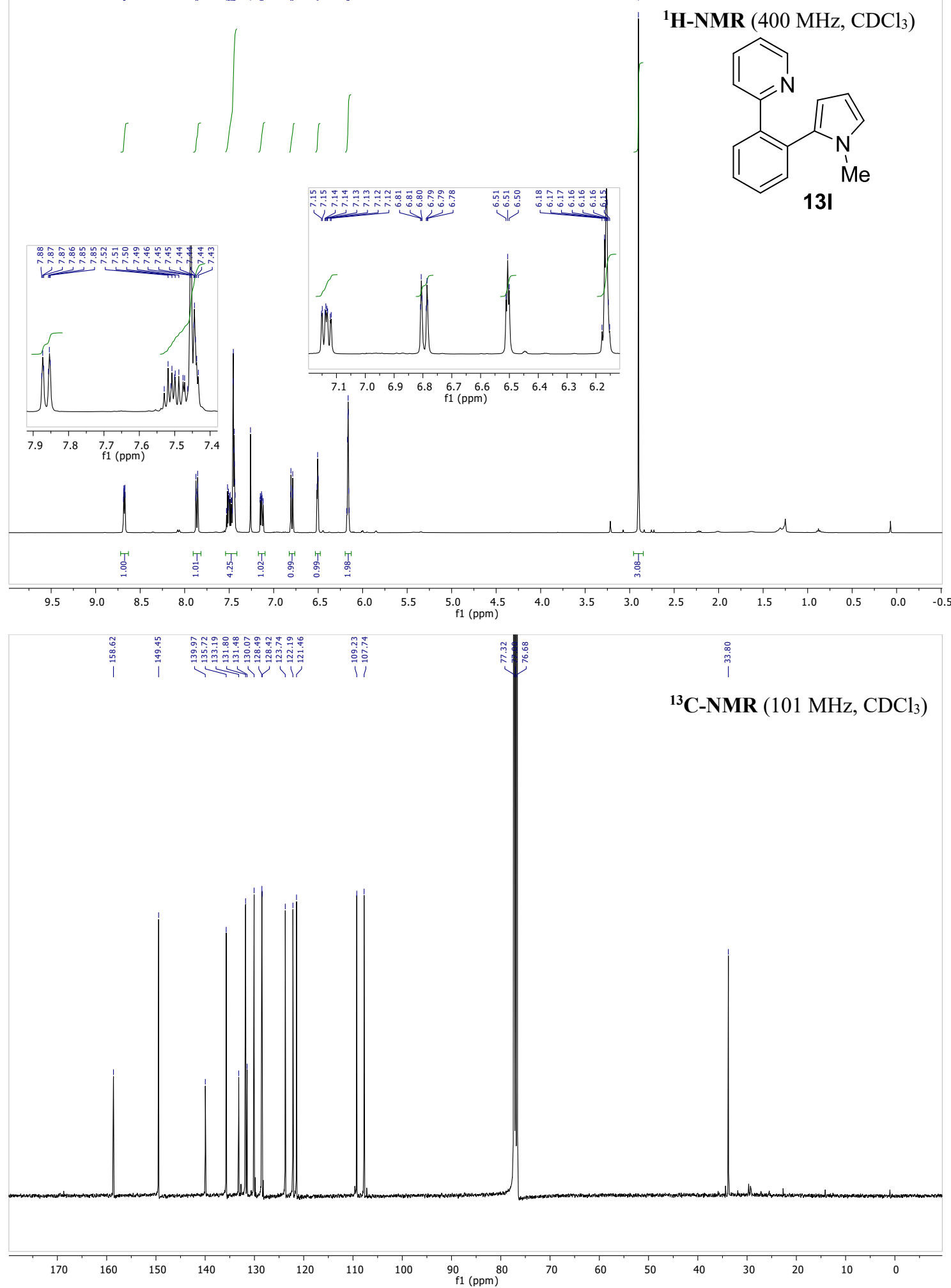


\section{2-(2-(1-Benzyl-1H-pyrrol-2-yl)phenyl)pyridine (13m)}

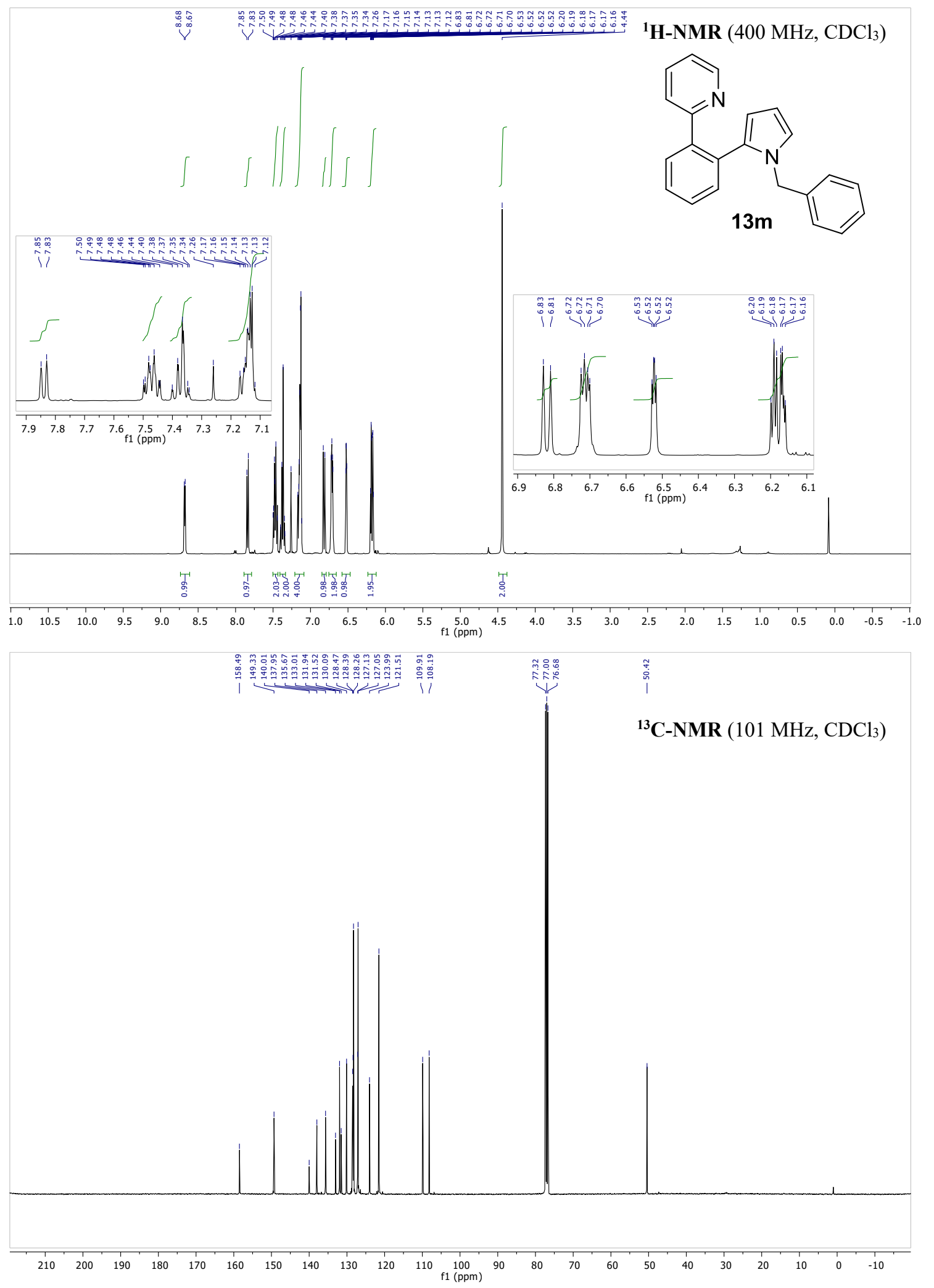


3-(5-Methoxy-2-(pyridin-2-yl)phenyl)-1-methyl-1H-indole (13n)
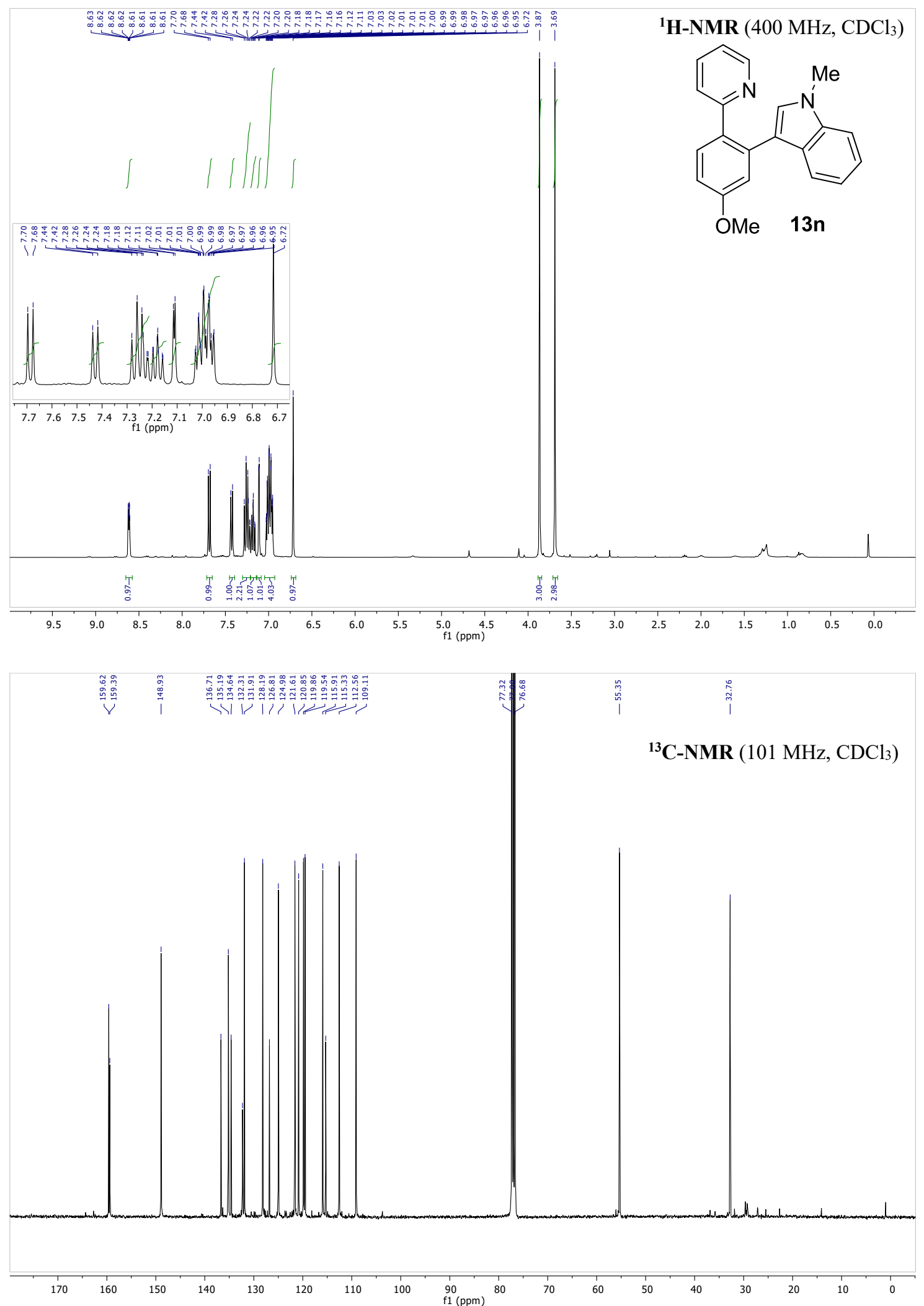
3-(5-Chloro-2-(pyridin-2-yl)phenyl)-1-methyl-1H-indole (130)
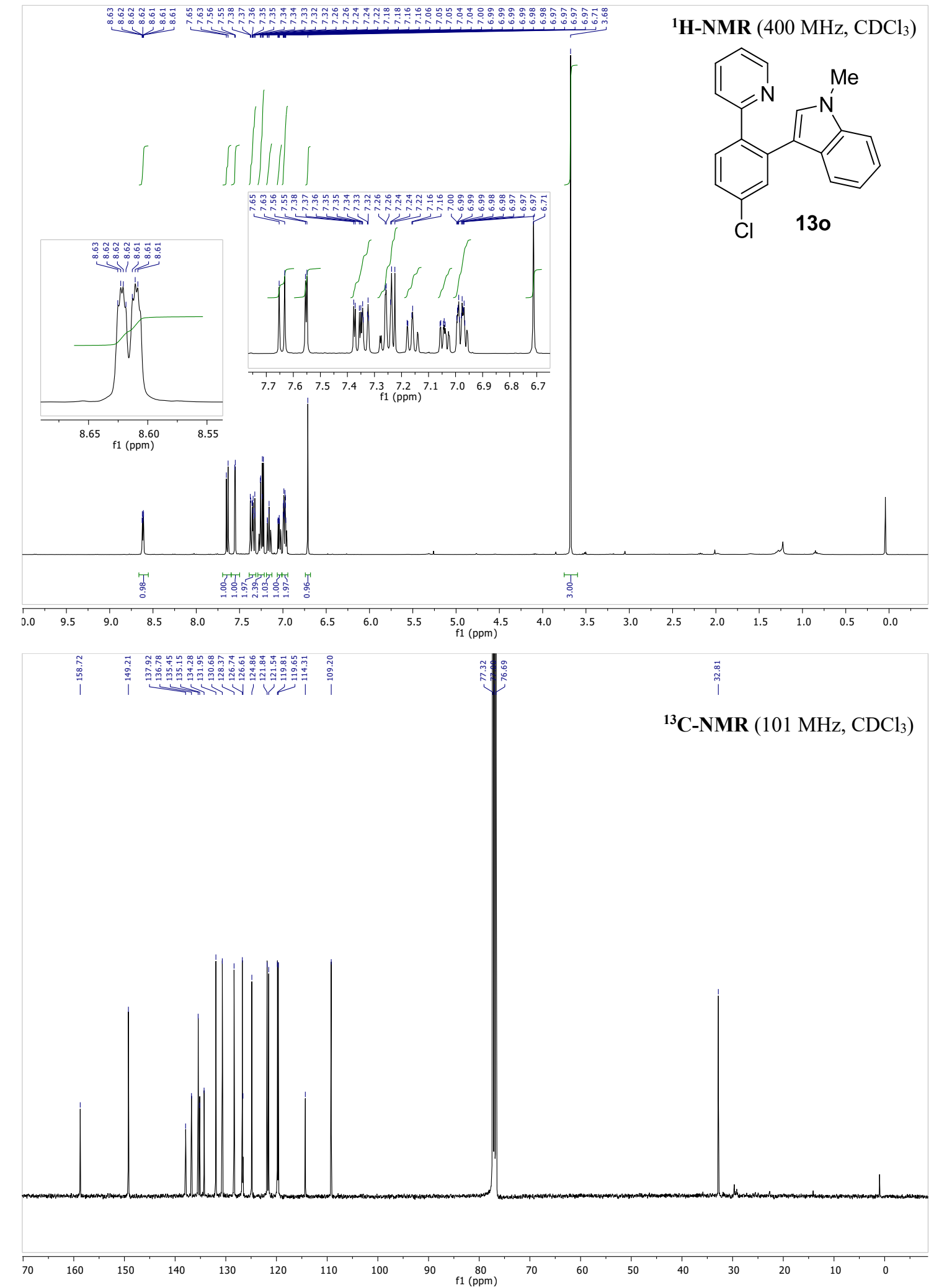
3-(5-Bromo-2-(pyridin-2-yl)phenyl)-1-methyl-1 $H$-indole (13p)

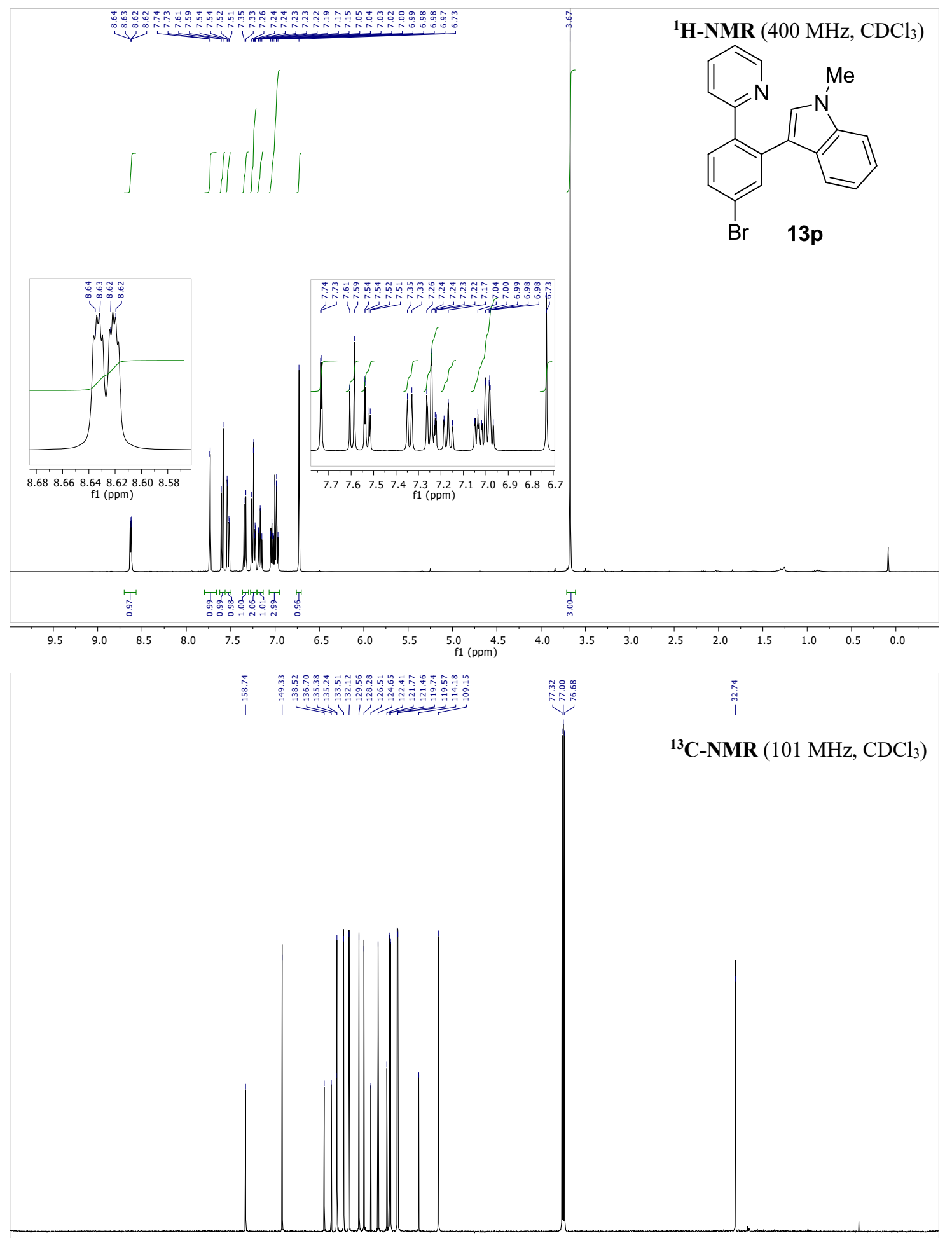

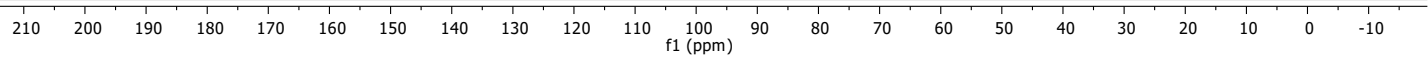


Methyl 3-(1-methyl-1H-indol-3-yl)-4-(pyridin-2-yl)benzoate (13q)

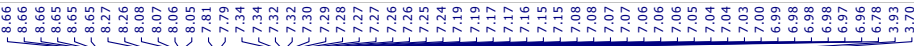
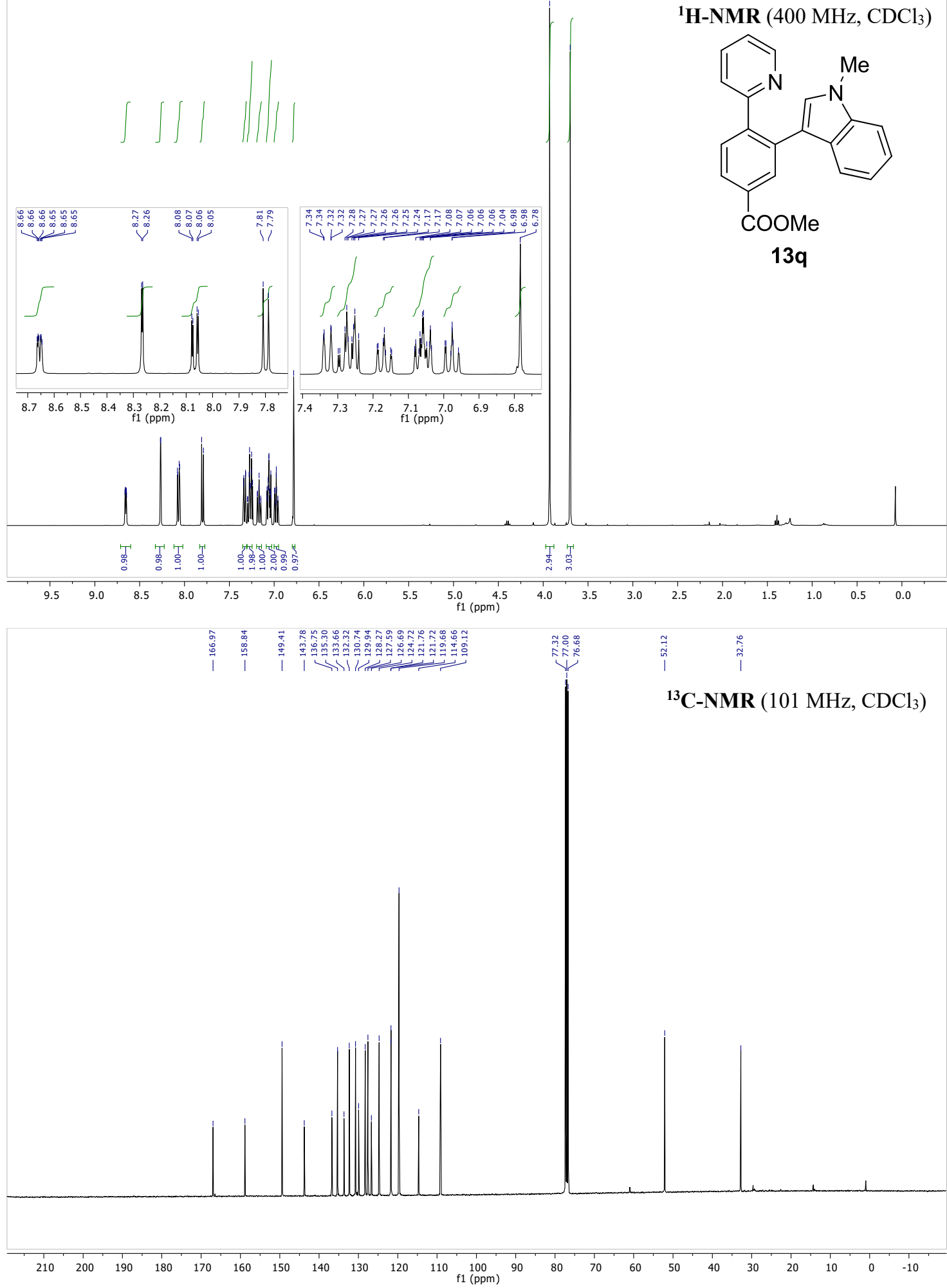


\section{3-(1-Methyl-1H-indol-3-yl)-4-(pyridin-2-yl)benzonitrile (13r)}
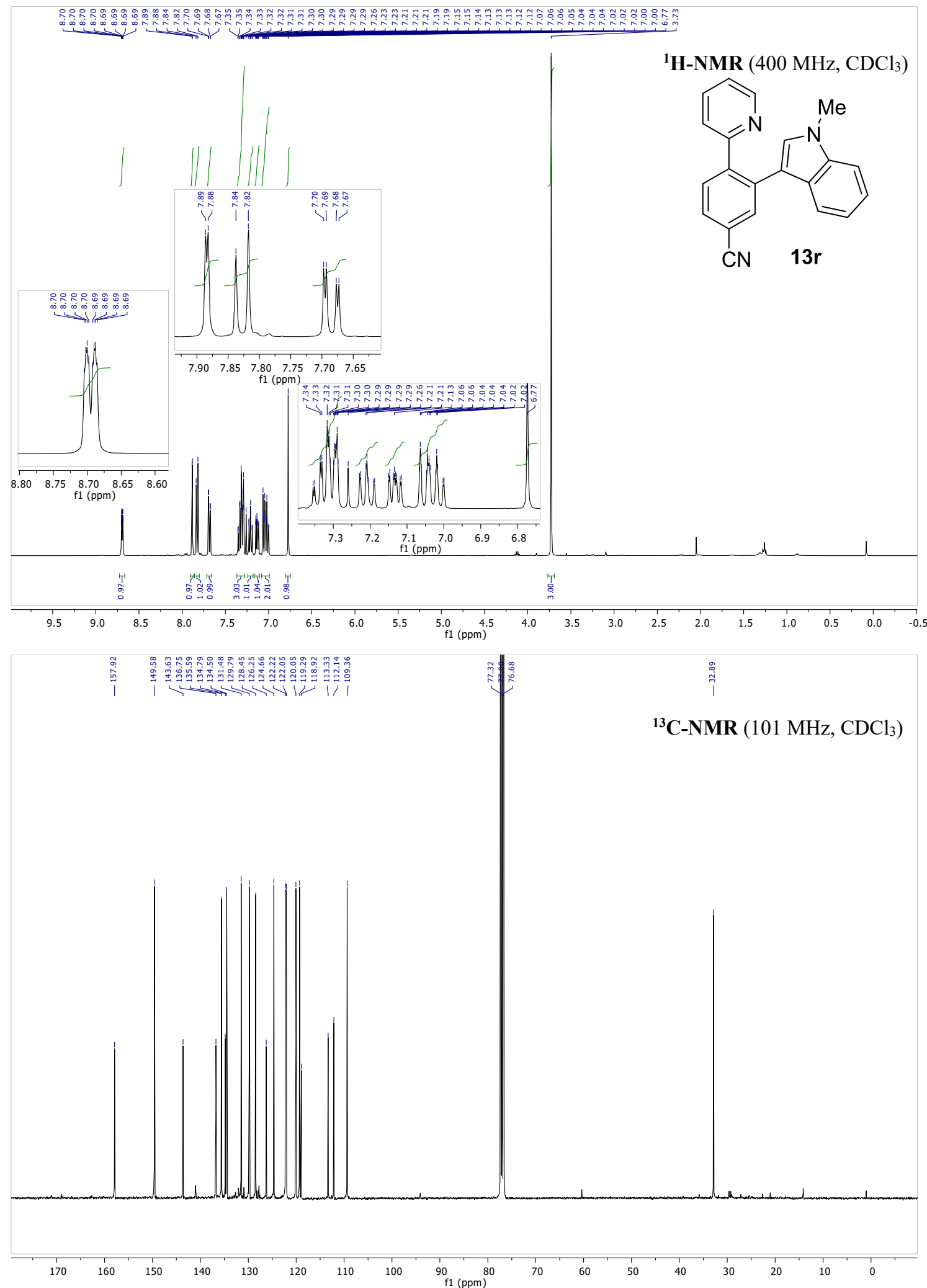
Methyl 3,5-bis(1-methyl-1H-indol-3-yl)-4-(pyridin-2-yl)benzoate (19)

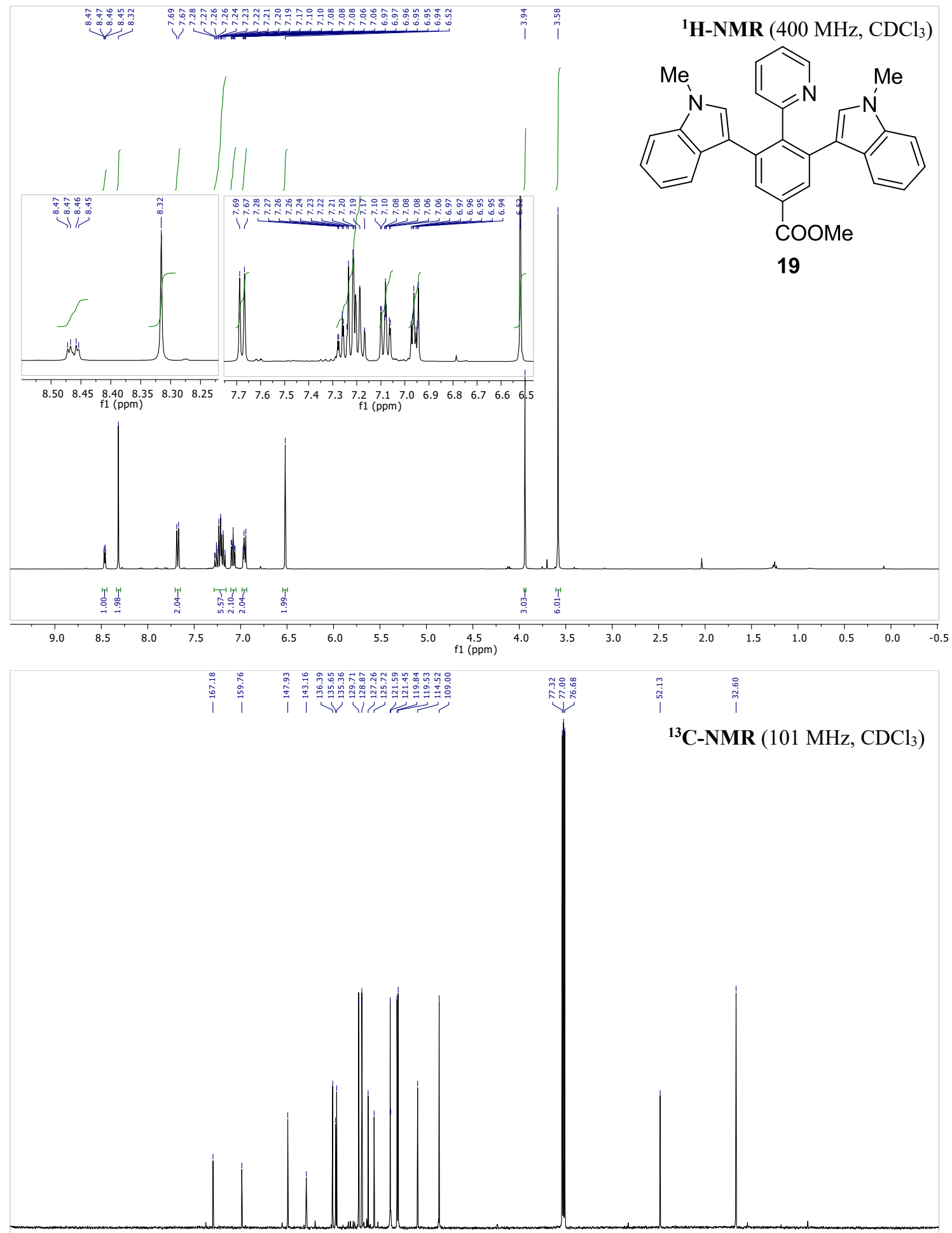

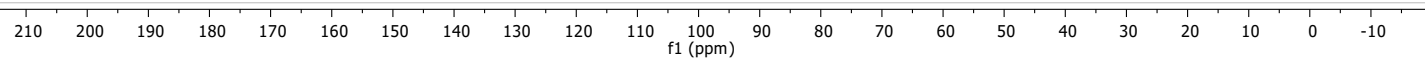


1-Methyl-3-(2-(pyrimidin-2-yl)phenyl)-1H-indole (20)

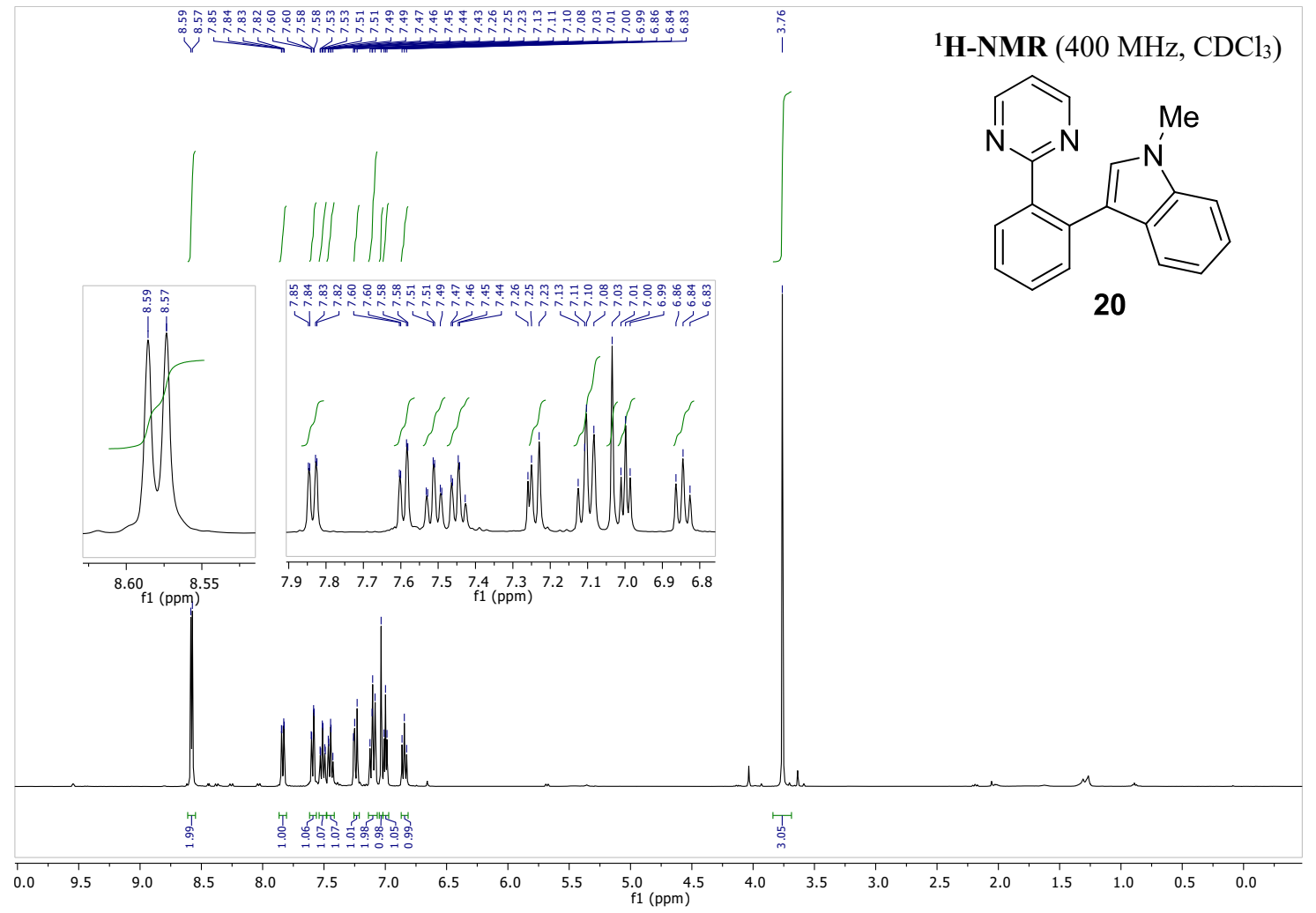

${ }^{13} \mathbf{C}-\mathrm{NMR}\left(101 \mathrm{MHz}, \mathrm{CDCl}_{3}\right)$

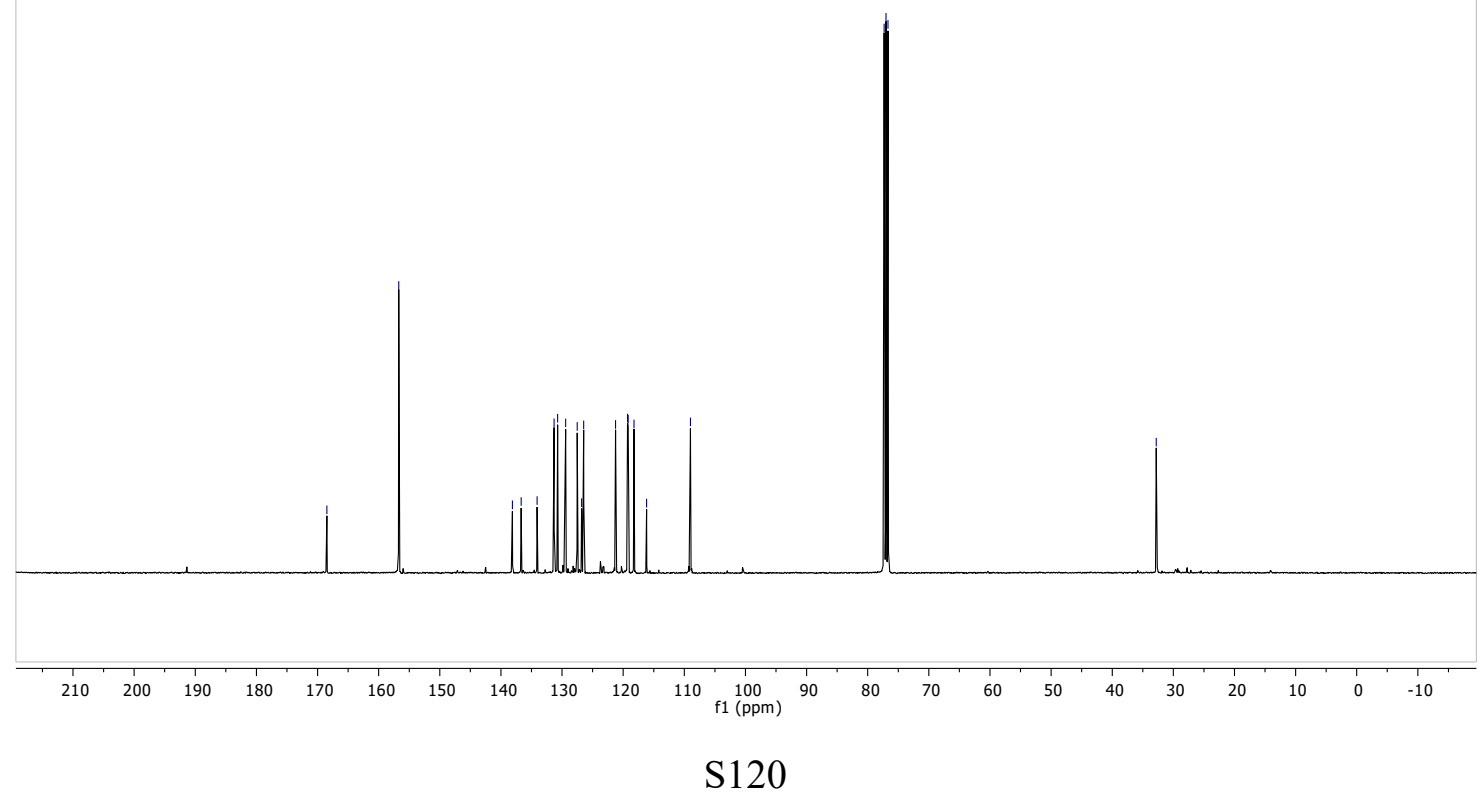


3-(2-(1H-Pyrazol-1-yl)phenyl)-1-methyl-1 $H$-indole (21)
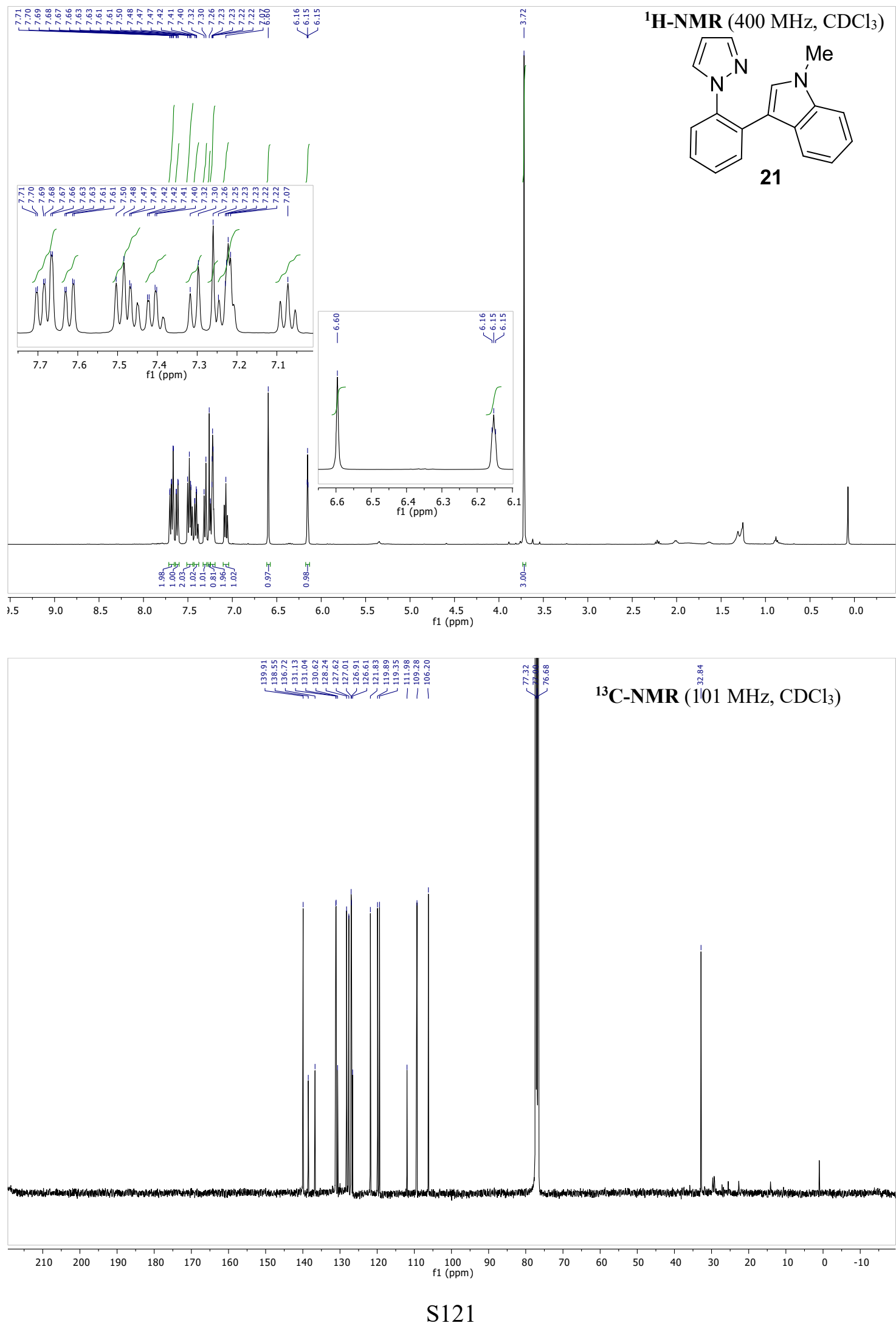
10-(1-Methyl-1 $H$-indol-3-yl)benzo[h]quinoline (22)

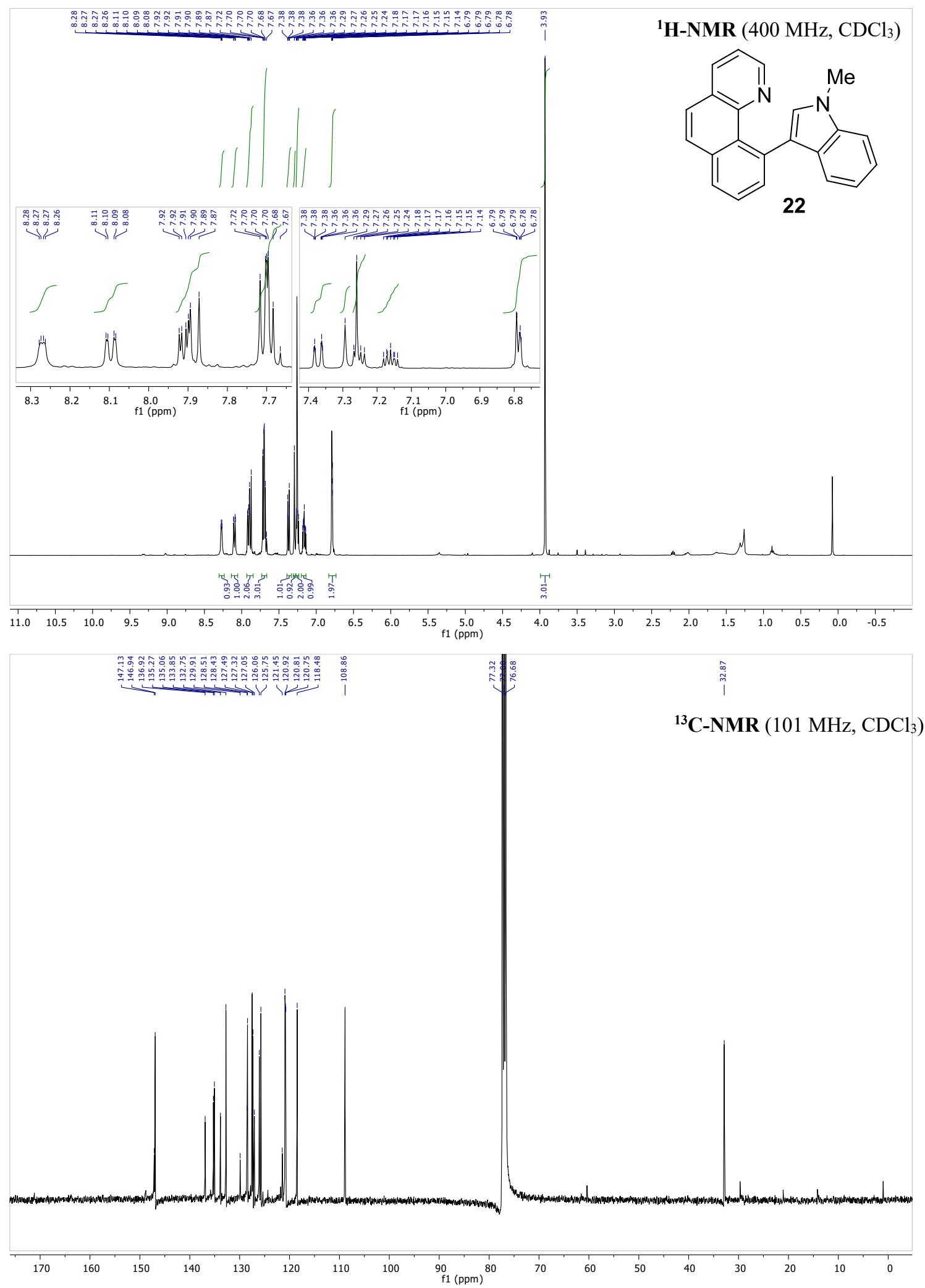


1'-Methyl-1-(pyrimidin-2-yl)-1H,1'H-2,3'-biindole (23)

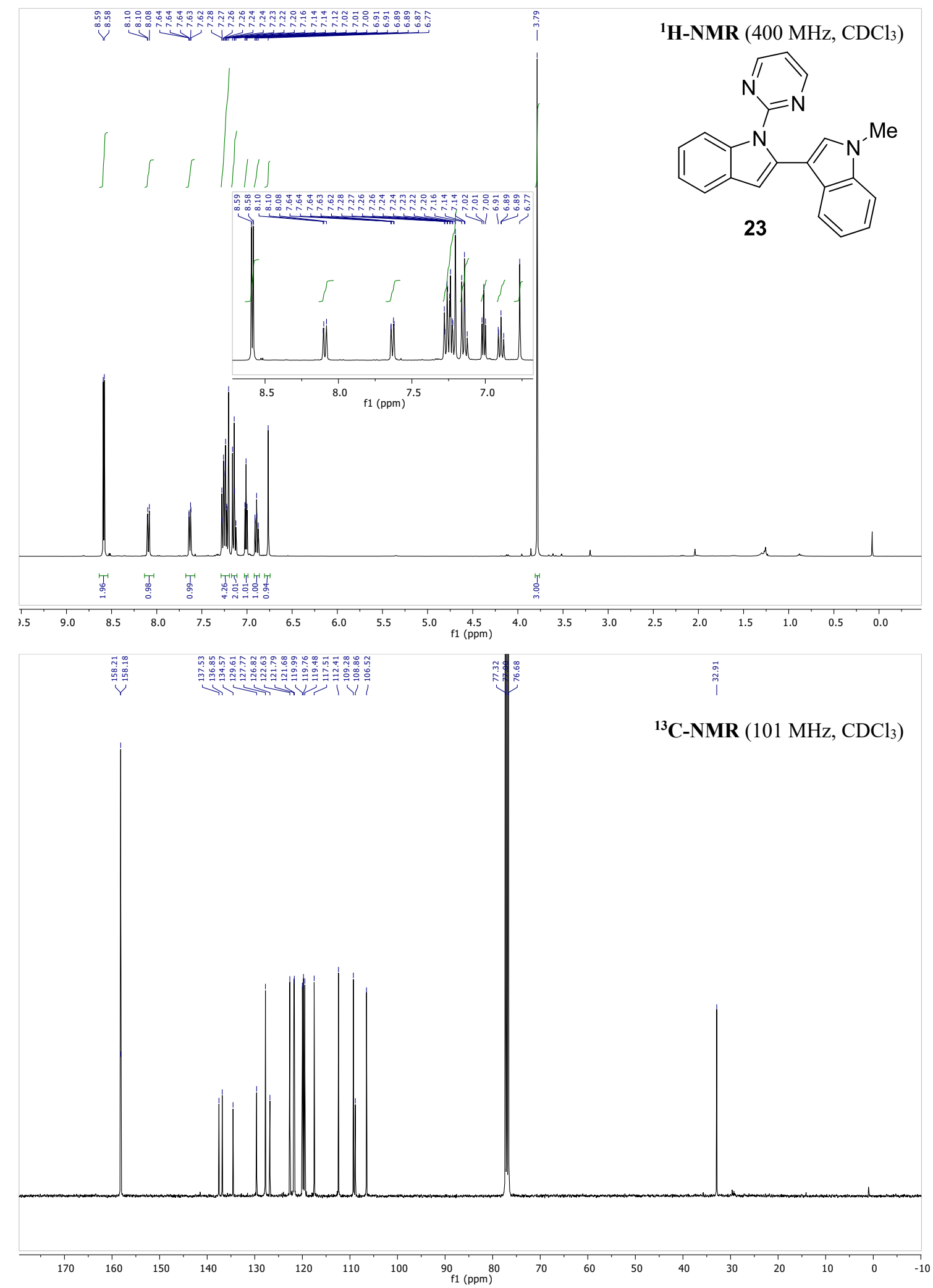


8-(1-Methyl-1H-indol-3-yl)quinolin-2(1H)-one (24)
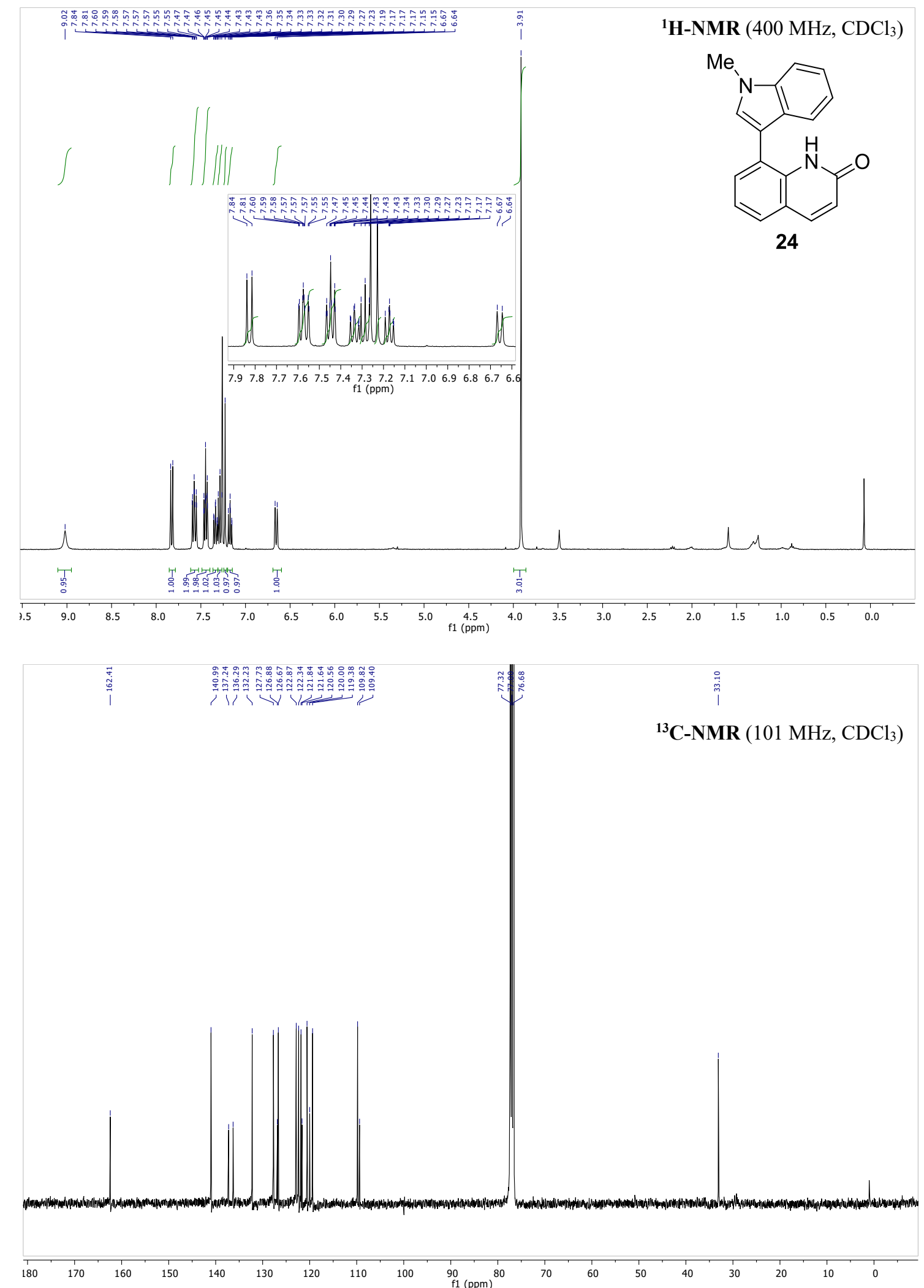
(2R,3R,4R,5R)-2-(Acetoxymethyl)-5-(6-(2-(1-methyl-1H-indol-3-yl)phenyl)-9H-purin-9yl)tetrahydrofuran-3,4-diyl diacetate (25)

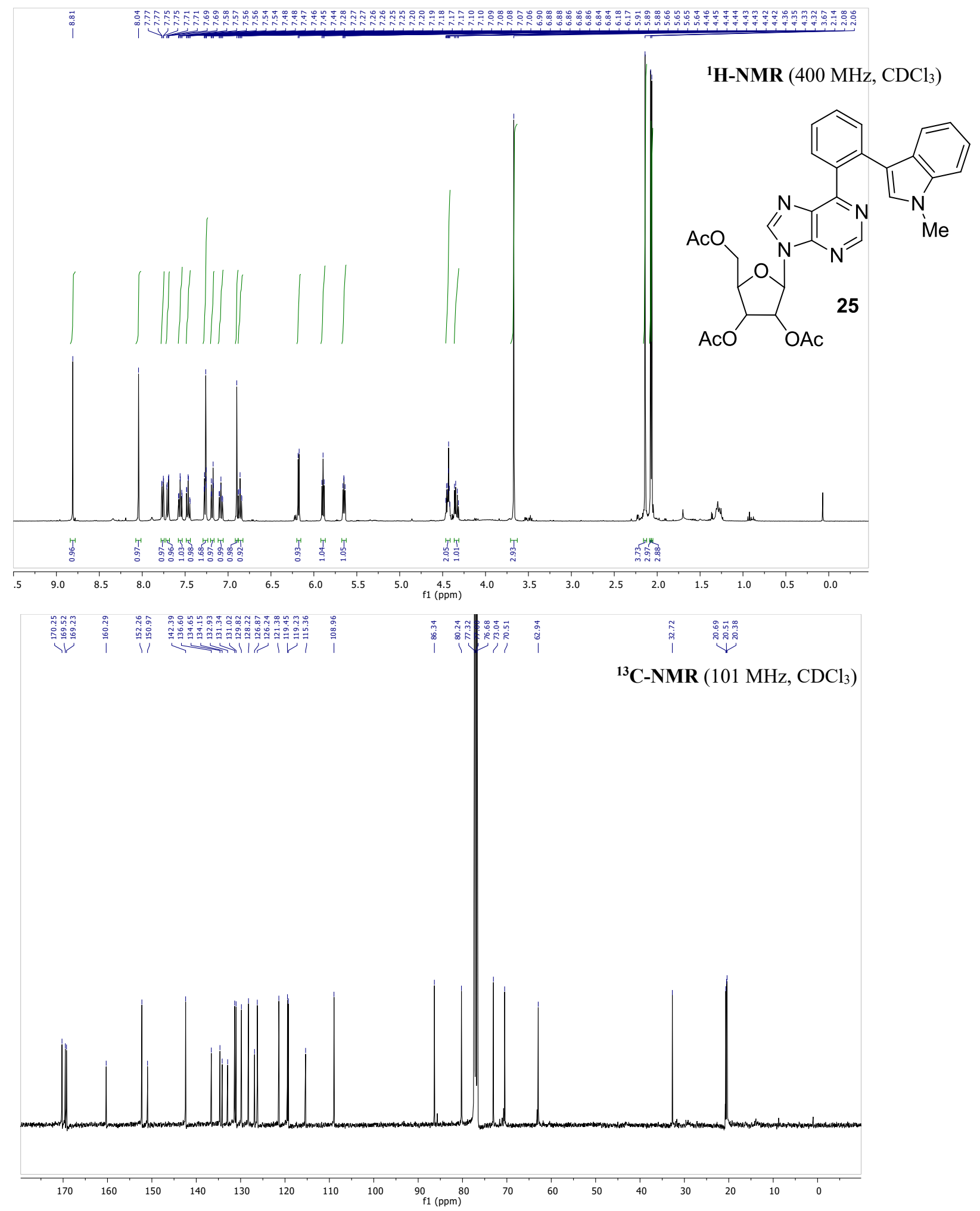

$\mathrm{S} 125$ 
N-Methoxy-2-(1-methyl-1H-indol-3-yl)benzamide (15a)
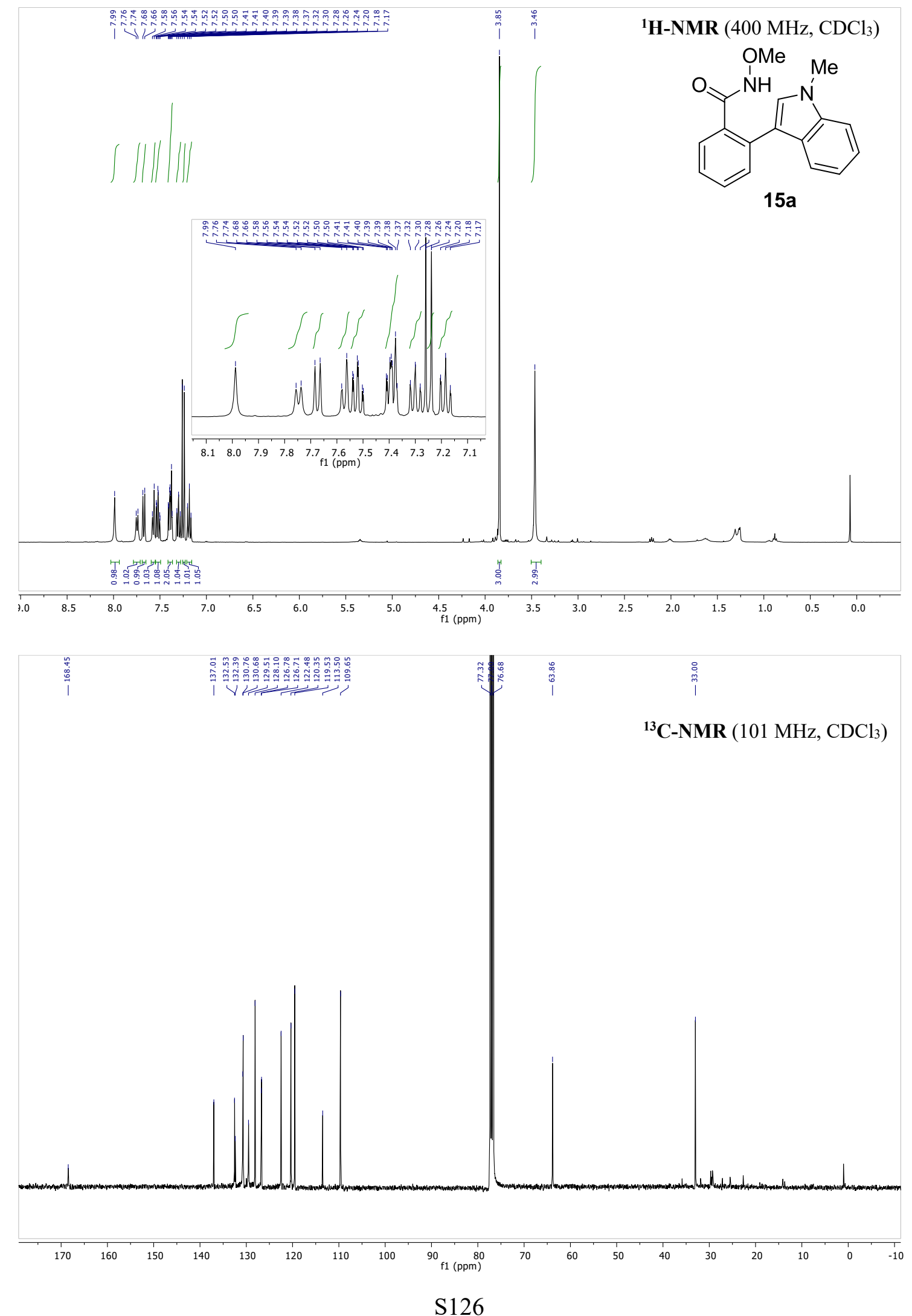
N-Methoxy-4-methyl-2-(1-methyl-1H-indol-3-yl)benzamide (15b)
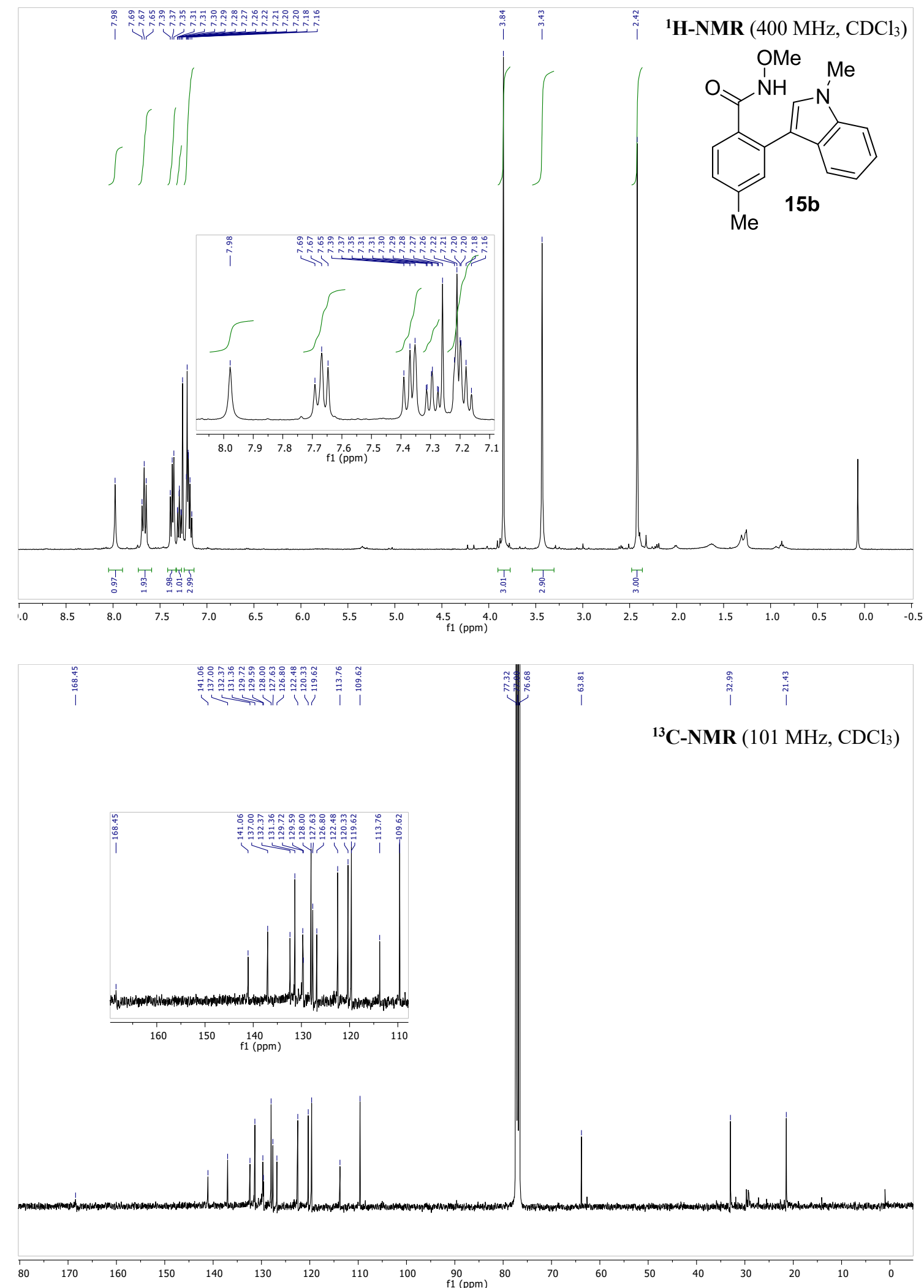

S127 
N,4-Dimethoxy-2-(1-methyl-1H-indol-3-yl)benzamide (15c)
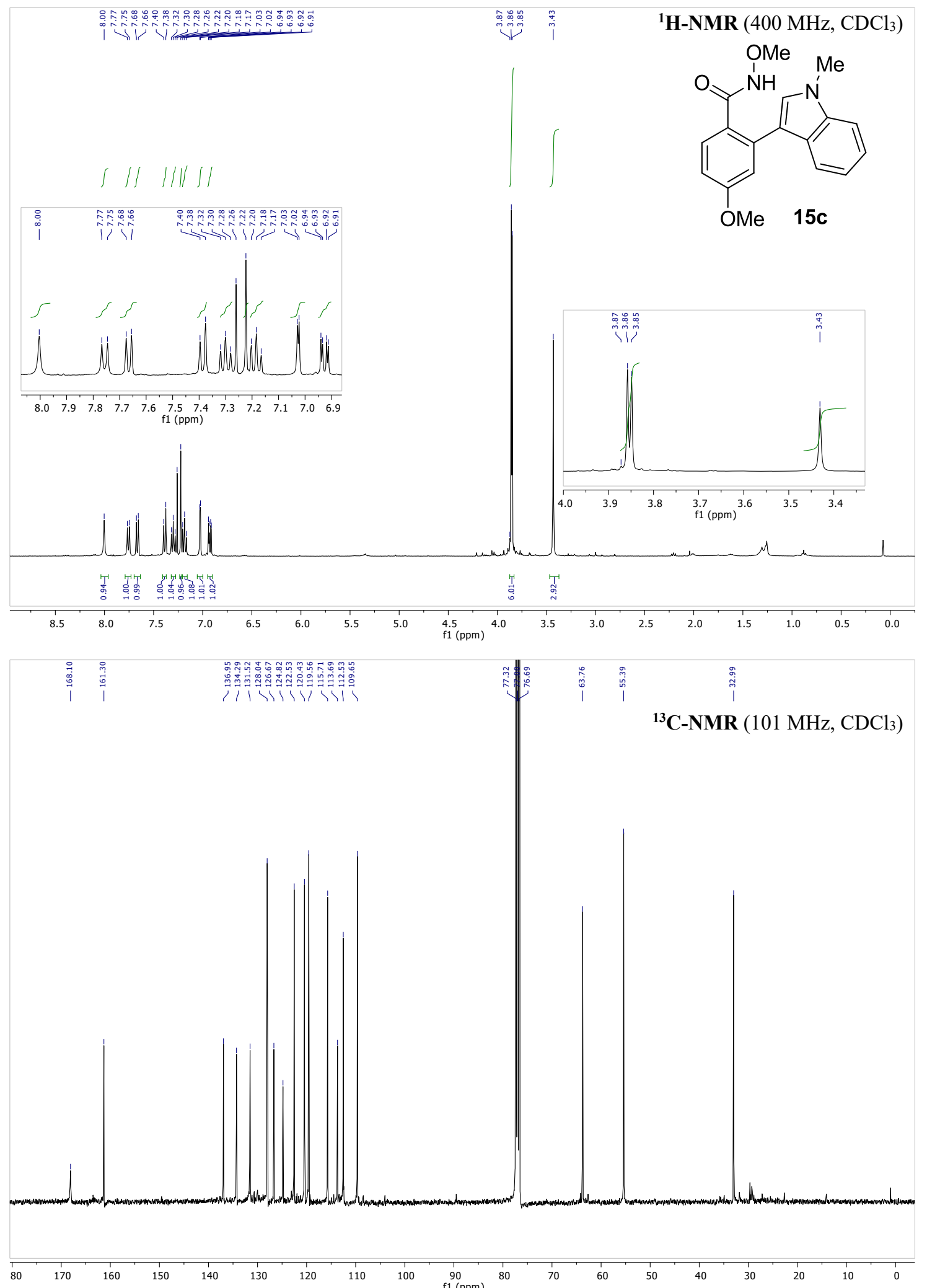
5-Fluoro-N-methoxy-2-(1-methyl-1H-indol-3-yl)benzamide (15d)
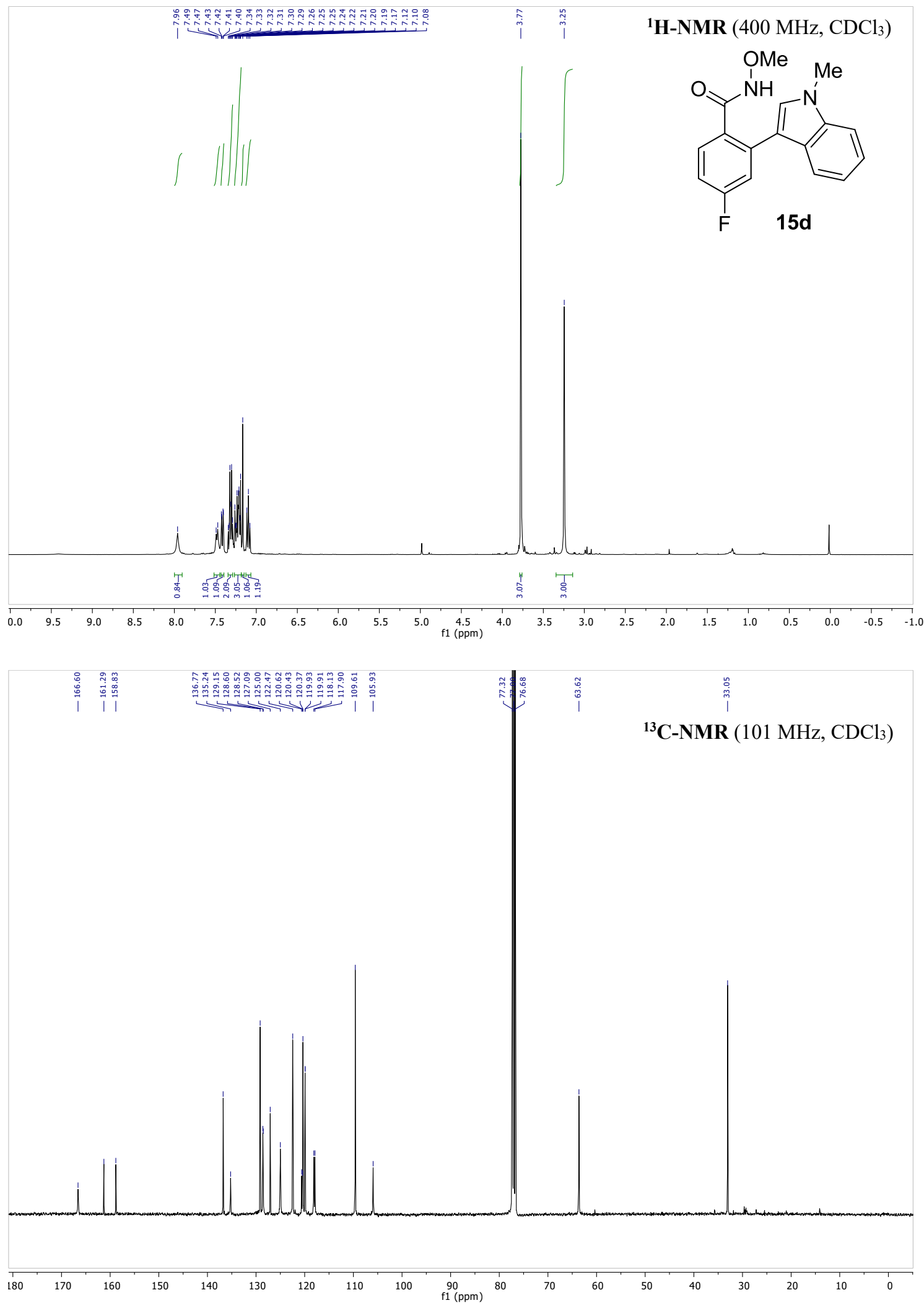

S129 

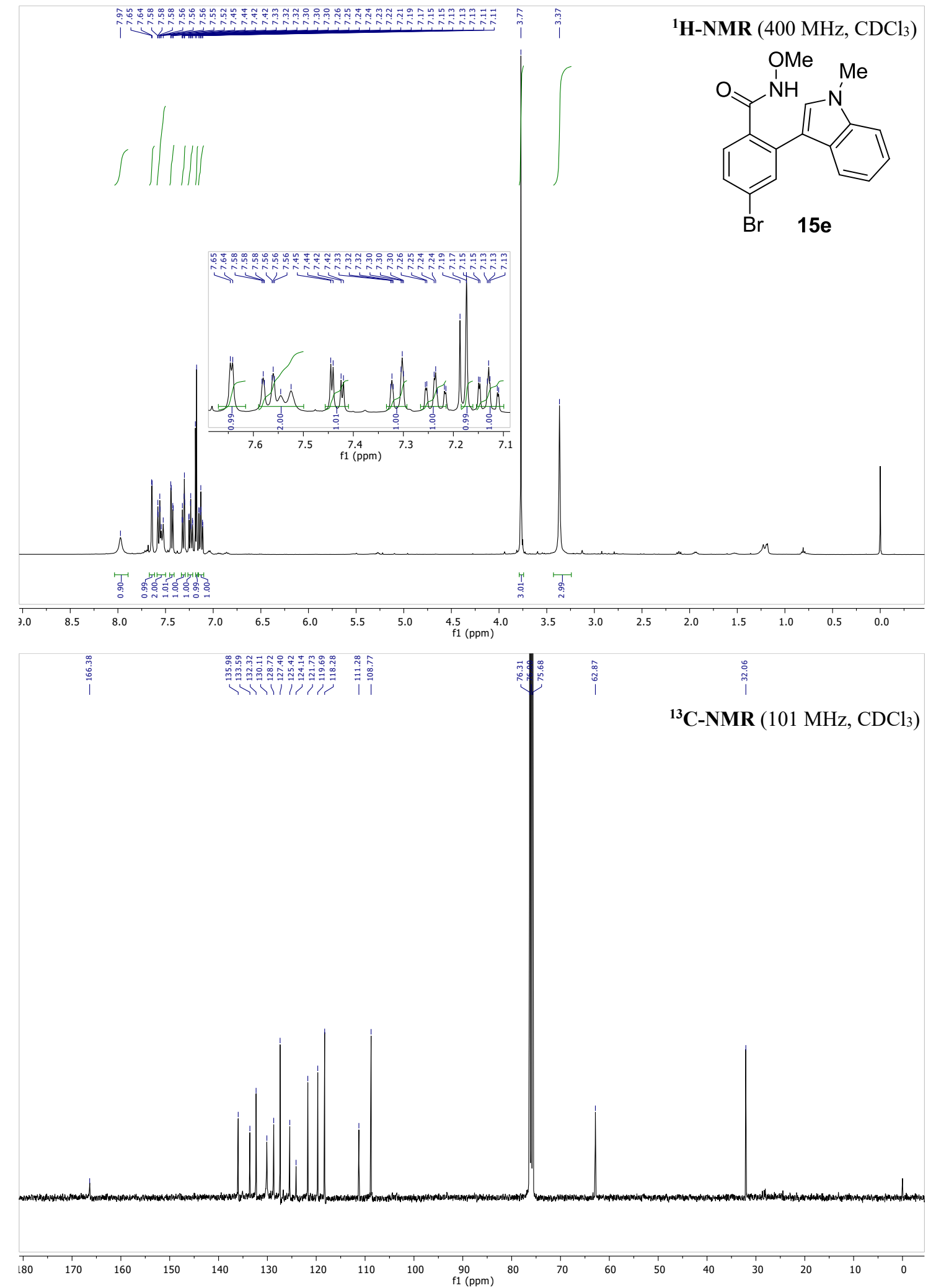
N-Methoxy-5-methyl-2-(1-methyl-1H-indol-3-yl)benzamide (15f)
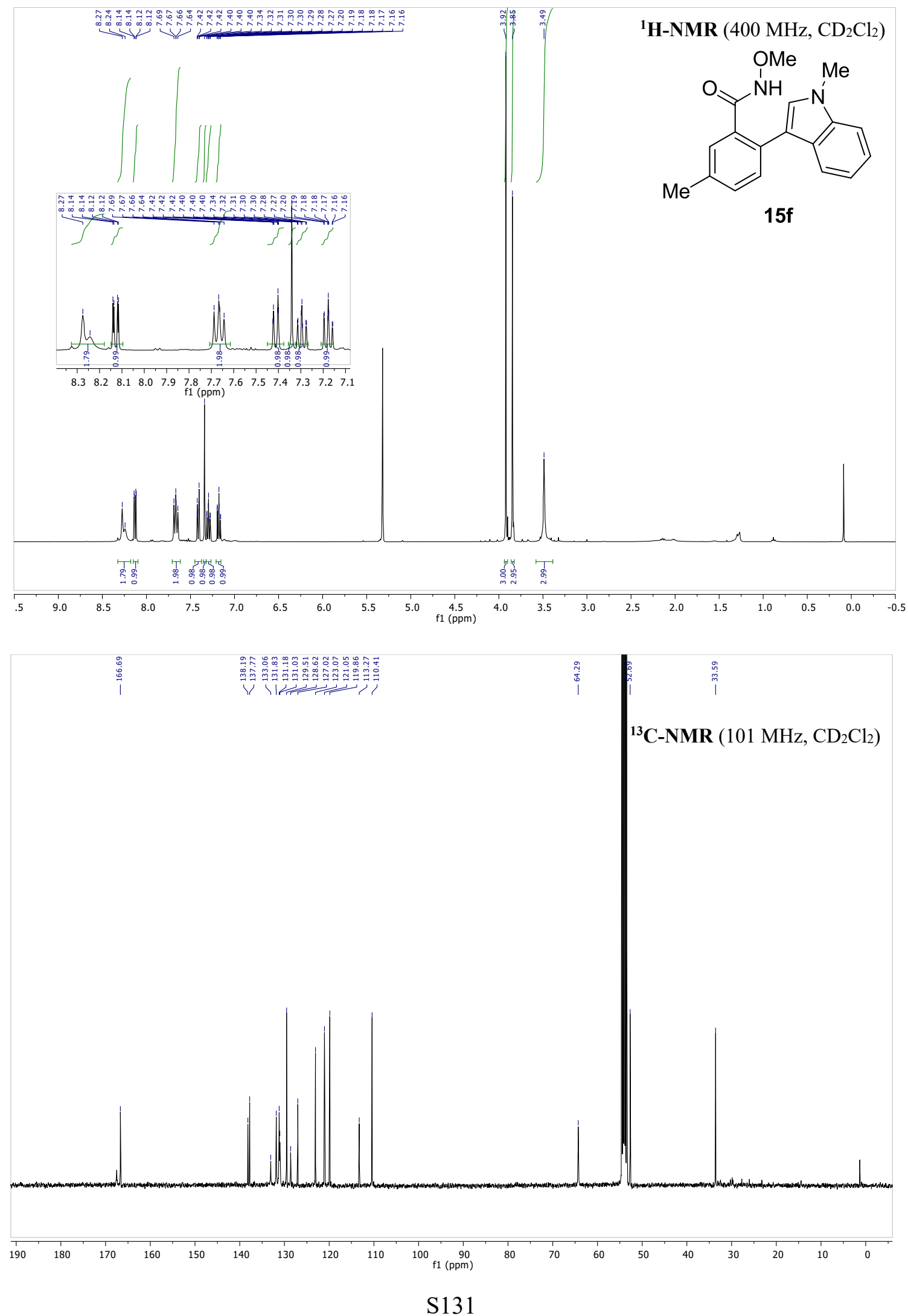
N-Methoxy-2-(1-methyl-1H-indol-3-yl)-5-(trifluoromethyl)benzamide (15g)

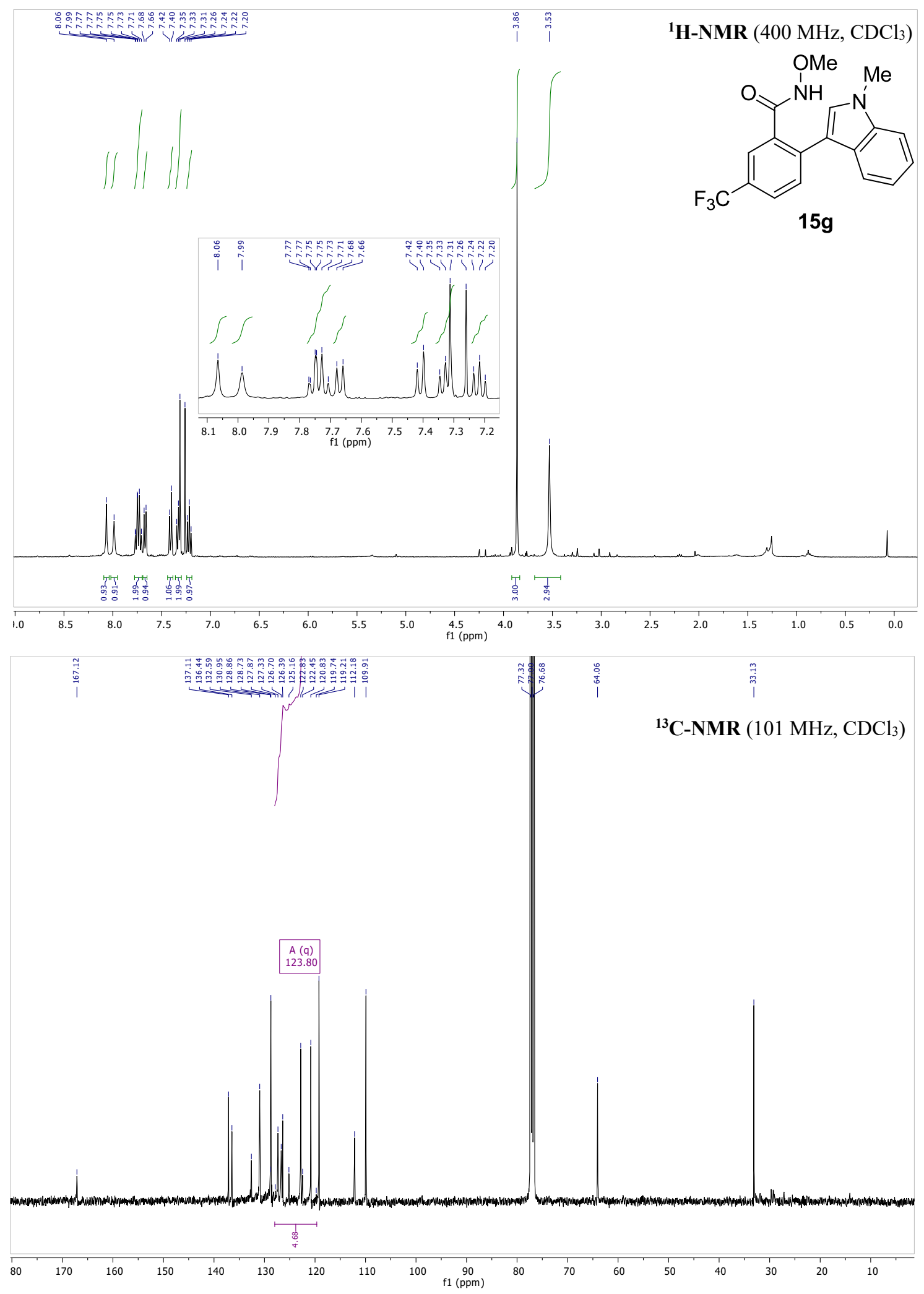


5-Fluoro-N-methoxy-2-(1-methyl-1H-indol-3-yl)benzamide (15h)

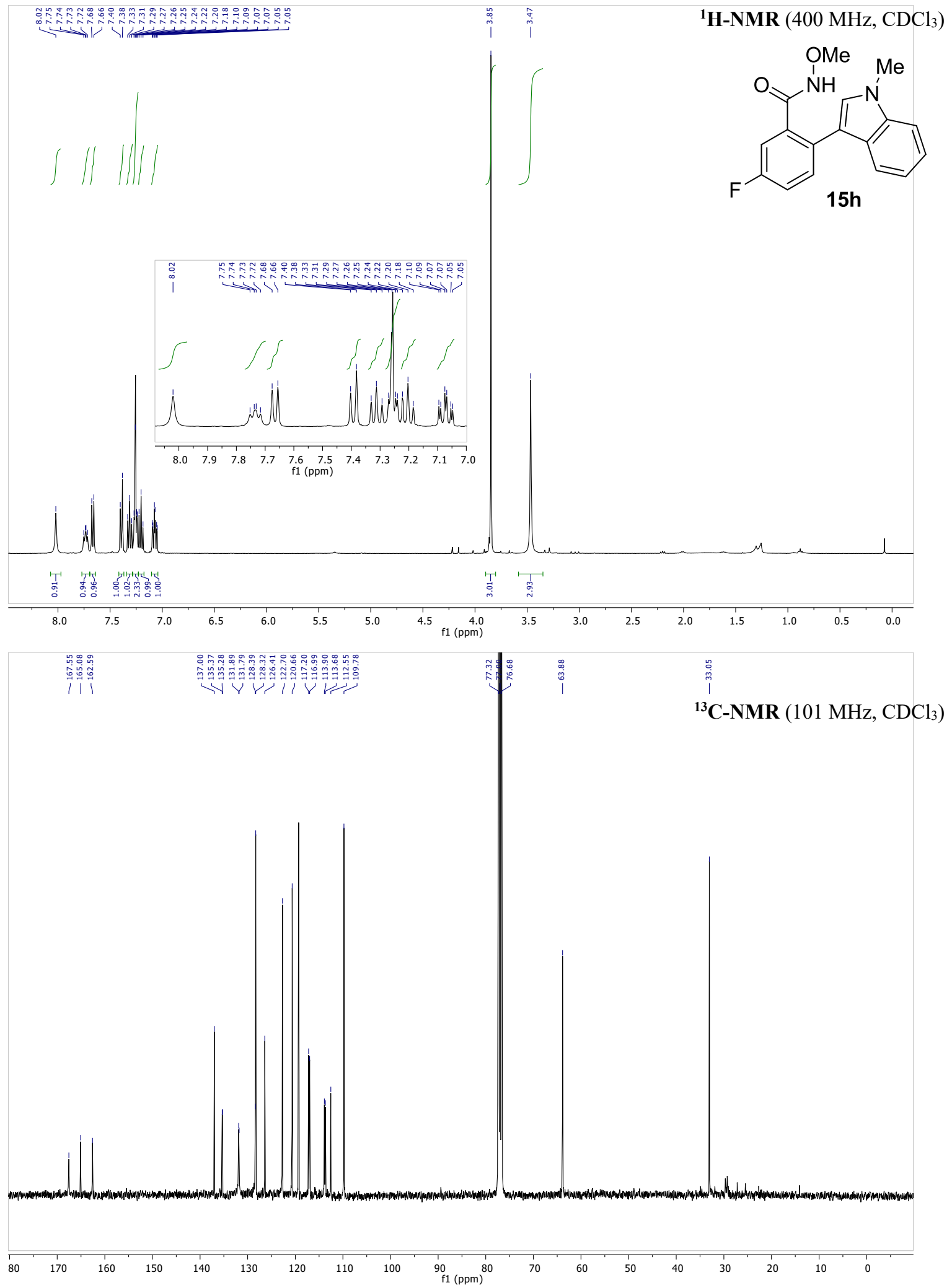


5-Chloro- $N$-methoxy-2-(1-methyl-1H-indol-3-yl)benzamide (15i)
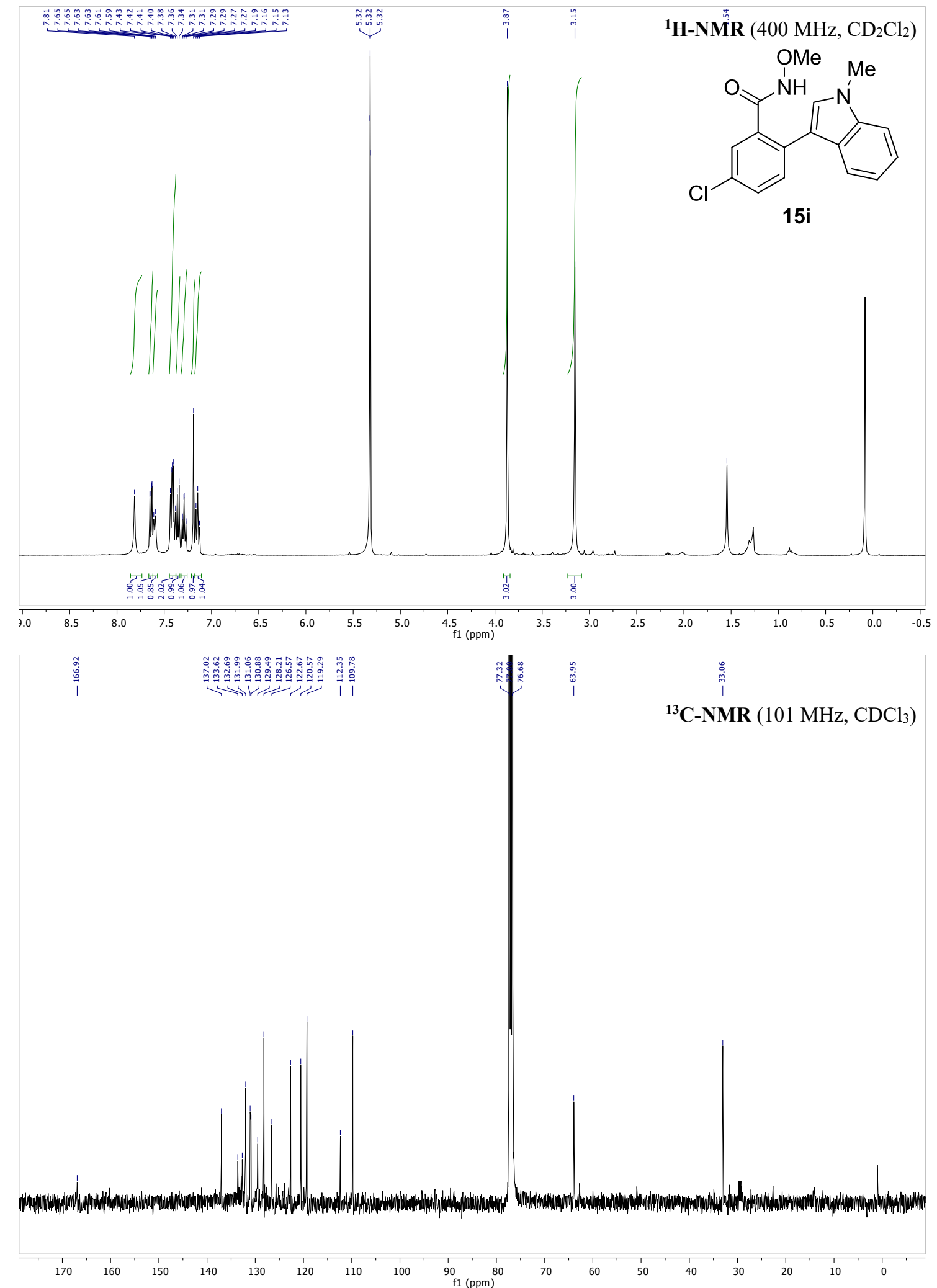
N-Methoxy-6-(1-methyl-1H-indol-3-yl)benzo[d][1,3]dioxole-5-carboxamide (15j)

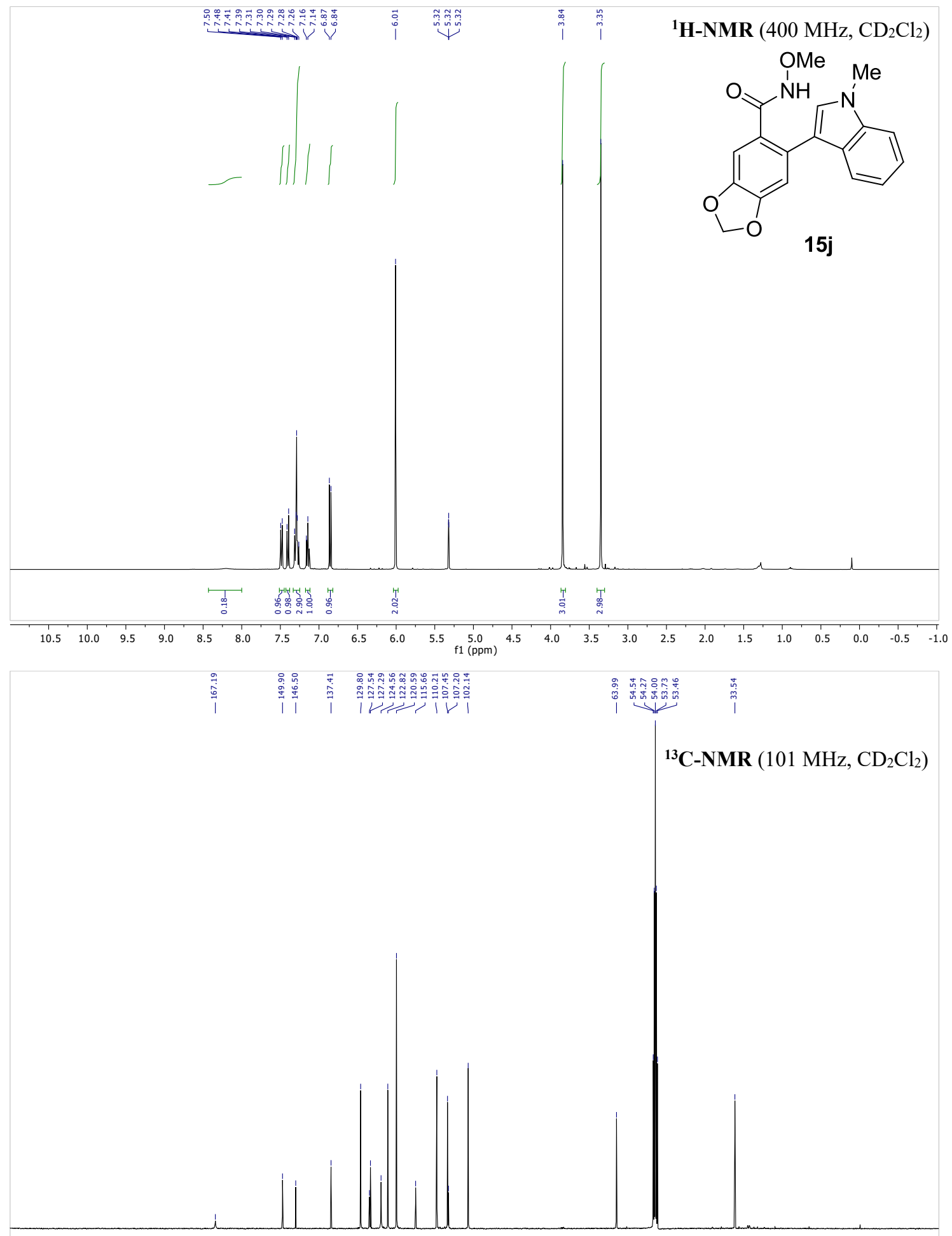

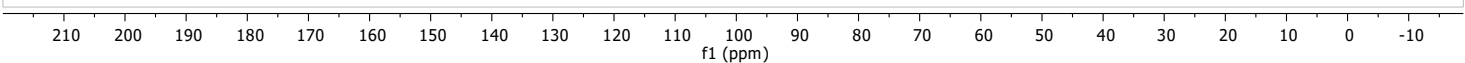


N-Methoxy-2-(1-methyl-1H-indol-3-yl)-1-naphthamide (15k)
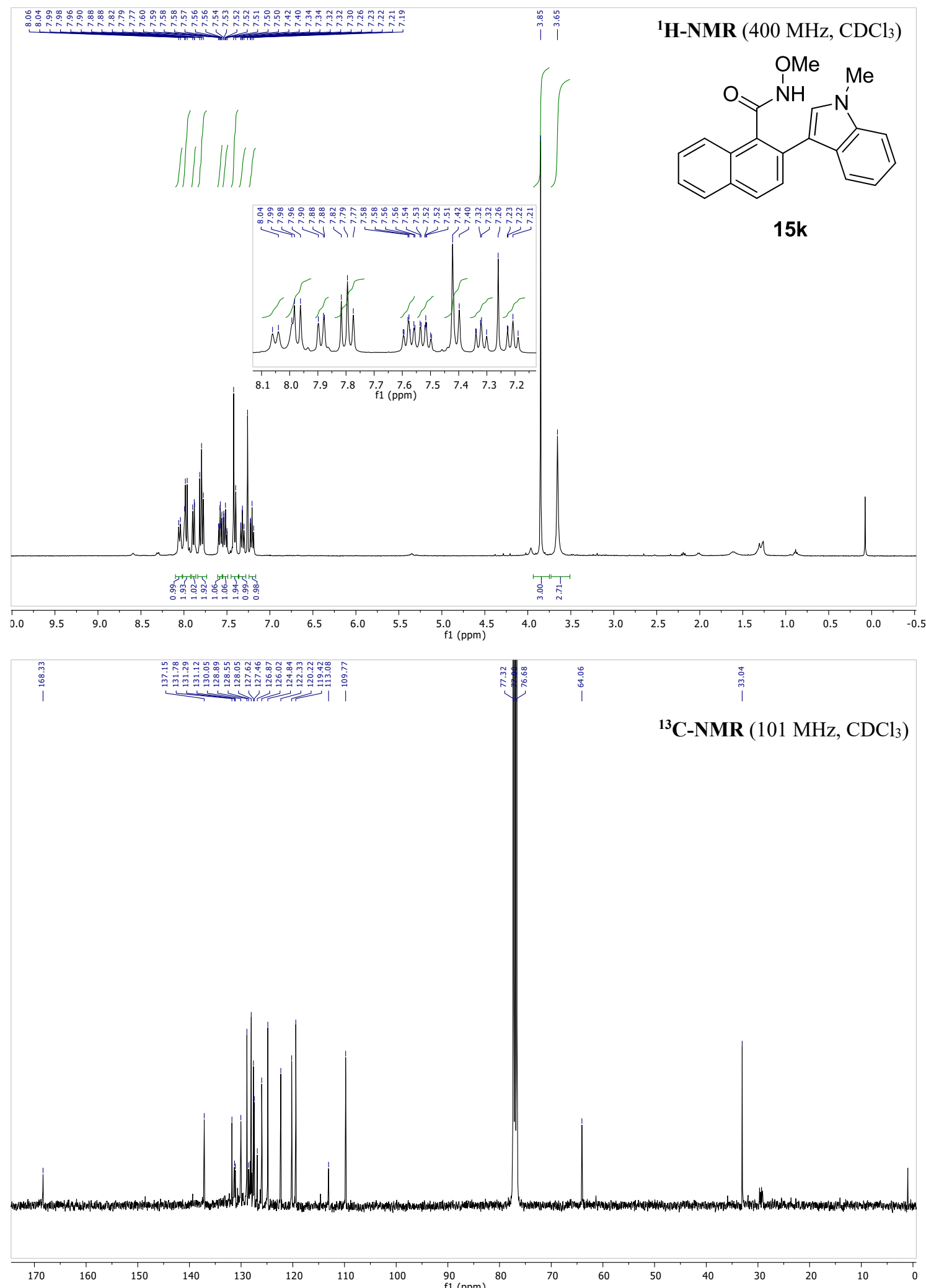
N-Methoxybenzo[d][1,3]dioxole-5-carboxamide (61)
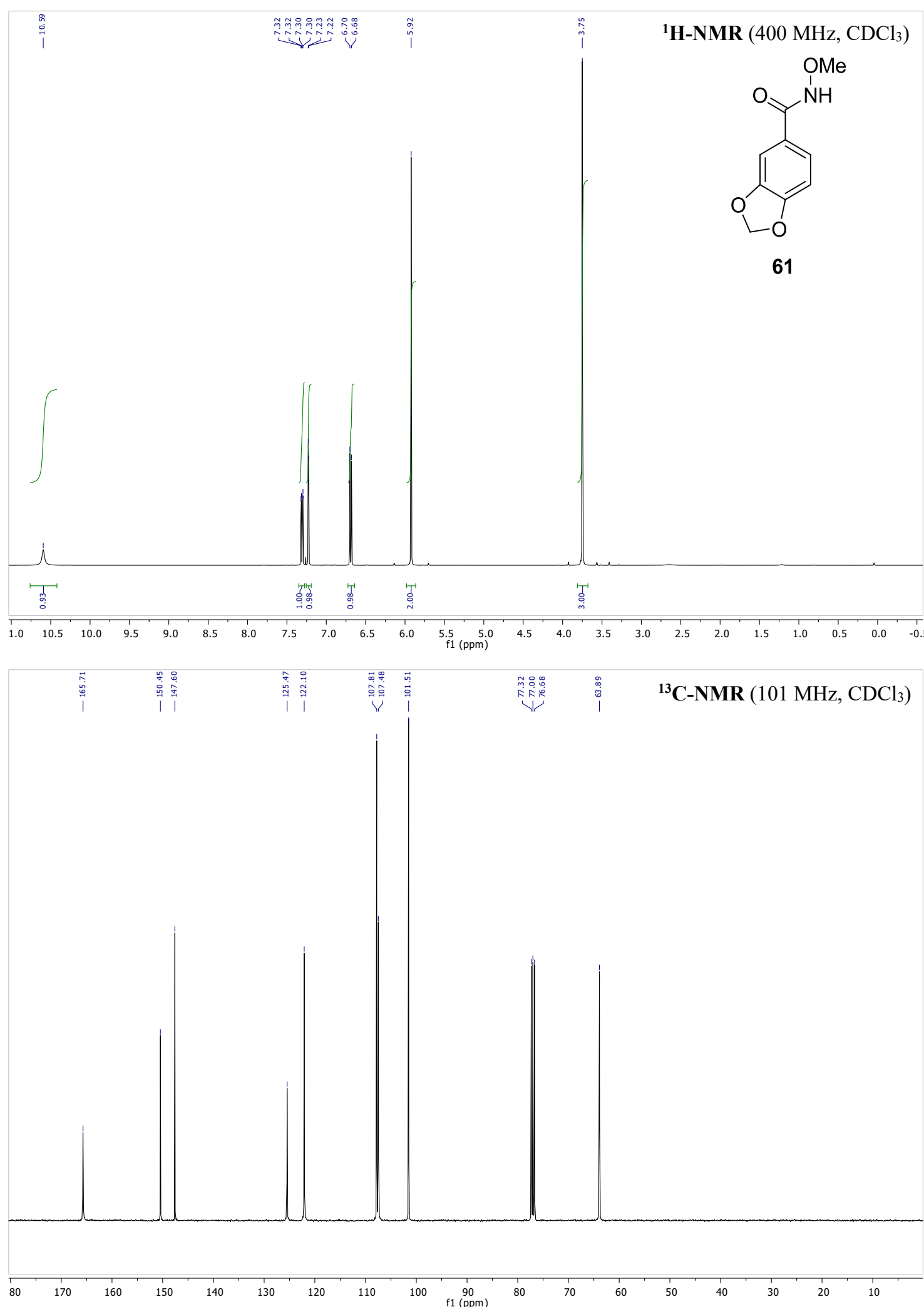